\title{
Lagrangian Reduction by Stages
}

\author{
Memoirs of the American Mathematical Society
}

152, no. 722, July 2001, 108 pp.

(Recieved by the AMS, April, 1999; Updated January 22, 2009).

\author{
Hernán Cendra \\ Departamento de Matemática \\ Universidad Nacional del Sur, Av. Alem 12548000 Bahia Blanca, Argentina \\ uscendra@criba.edu.ar ${ }^{1}$ \\ Jerrold E. Marsden \\ Control and Dynamical Systems 107-81 \\ California Institute of Technology Pasadena, CA 91125 \\ marsden@cds.caltech.edu ${ }^{2}$ \\ Tudor S. Ratiu \\ Département de Mathématiques \\ École Polytechnique Fédérale de Lausanne CH 1015 Lausanne, Switzerland \\ Tudor.Ratiu@epfl.ch ${ }^{3}$
}

1 October 2009

\footnotetext{
${ }^{1}$ The research of $\mathrm{HC}$ was partly done during a sabbatical stay in Control and Dynamical Systems at the California Institute of Technology.

${ }^{2}$ The research of JEM was supported in part by the California Institute of Technology and NSF Grants DMS-9802106 and ATM-9873133.

${ }^{3}$ The research of TSR was supported in part by NSF Grant DMS-9802378 and FNS Grant 21-54138.98.
} 


\section{Contents}

Preface $\quad$ v

1 Introduction 1

1.1 Background . . . . . . . . . . . . . . . . 2

1.2 The Main Results of This Paper. . . . . . . . . . . . . . . . . 3

1.3 Future Work and Related Issues. . . . . . . . . . . . . . . . . . 4

2 Preliminary Constructions $\quad 7$

2.1 Notation and general assumptions. . . . . . . . . . . . 7

2.2 Connections on Principal Bundles. . . . . . . . . . . . . . . . 9

2.3 Associated Bundles. . . . . . . . . . . . . . . . . . . 11

2.4 The Bundles $T Q / G$ and $T(Q / G) \oplus \tilde{\mathfrak{g}} \ldots \ldots \ldots \ldots$

3 The Lagrange-Poincaré Equations 19

3.1 The Geometry of Variations . . . . . . . . . . . . . . . . . . . . 19

3.2 The Euler-Lagrange and Euler-Poincaré Operators . . . . . . . . . 25

3.3 The Lagrange-Poincaré Operator . . . . . . . . . . . . . . . . 33

3.4 The Reduced Variational Principle . . . . . . . . . . . . . . . . 39

4 Wong's Equations and Coordinate Formulas 43

4.1 Wong's Equations . . . . . . . . . . . . . . . . . . . . 43

4.2 The Local Vertical and Horizontal Equations . . . . . . . . . . . 45

5 The Lie Algebra Structure on Sections of the Reduced Bundle 51

5.1 The Bundle $T(Q / G) \oplus \tilde{\mathfrak{g}}$ Revisited . . . . . . . . . . . 51 


\section{iv Contents}

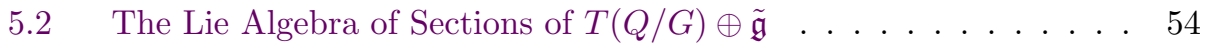

6 Reduced Tangent Bundles $\quad 61$

6.1 The Geometry of Lagrange-Poincaré Bundles . . . . . . . . . . . . 62

6.2 Reduction of Lagrange-Poincaré Bundles . . . . . . . . . . . . . . . 73

6.3 Reduction by Stages of objects of $\mathfrak{L P} \ldots \ldots \ldots$. . . . . . . . 84

6.4 The Subcategory $\mathfrak{R T}$ and Reduction by Stages . . . . . . . . . . 92

7 Further Examples $\quad 101$

7.1 Semidirect Products . . . . . . . . . . . . . . . . . 101

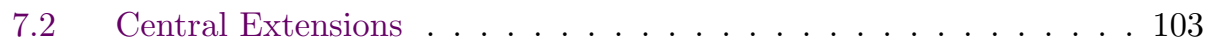

7.3 Rigid Body with Rotors . . . . . . . . . . . . . . . . 104

7.4 Systems Depending on a Parameter . . . . . . . . . . . 105

8 The Category $\mathfrak{L P}^{*}$ and Poisson Geometry 111

8.1 The Poisson Bracket on Duals of Objects of $\mathfrak{L P}$. . . . . . . . . . 111

8.2 Poisson Reduction in the Category $\mathfrak{L P}^{*}$. . . . . . . . . . . 117 


\section{Preface}

This booklet studies the geometry of the reduction of Lagrangian systems with symmetry in a way that allows the reduction process to be repeated; that is, it develops a context for Lagrangian reduction by stages. The Lagrangian reduction procedure focuses on the geometry of variational structures and how to reduce them to quotient spaces under group actions. This philosophy is well known for the classical cases, such as Routh reduction for systems with cyclic variables (where the symmetry group is Abelian) and Euler-Poincaré reduction (for the case in which the configuration space is a Lie group) as well as Euler-Poincaré reduction for semidirect products.

The context established for this theory is a Lagrangian analogue of the bundle picture on the Hamiltonian side. In this picture, we develop a category that includes, as a special case, the realization of the quotient of a tangent bundle as the Whitney sum of the tangent of the quotient bundle with the associated adjoint bundle. The elements of this new category, called the Lagrange-Poincaré category, have enough geometric structure so that the category is stable under the procedure of Lagrangian reduction. Thus, reduction may be repeated, giving the desired context for reduction by stages. Our category may be viewed as a Lagrangian analog of the category of Poisson manifolds in Hamiltonian theory.

We also give an intrinsic and geometric way of writing the reduced equations, called the Lagrange-Poincaré equations, using covariant derivatives and connections. In addition, the context includes the interpretation of cocycles as curvatures of connections and is general enough to encompass interesting situations involving both semidirect products and central extensions. Examples are given to illustrate the general theory. 
In classical Routh reduction one usually sets the conserved quantities conjugate to the cyclic variables equal to a constant. In our development, we do not require the imposition of this constraint. For the general theory along these lines, we refer to the complementary work of Marsden, Ratiu and Scheurle (2000), which studies the Lagrange-Routh equations.

Received by the editor April 12, 1999 and in revised form March 15, 2000.

1991 Mathematics Subject Classification. Primary 37J15; Secondary 70H33, 53D20.

Key words and phrases. Lagrangian reduction, mechanical systems, variational principles, symmetry. 


\section{1}

\section{Introduction}

Reduction theory for mechanical systems with symmetry has its origins in the classical works of Euler, Lagrange, Hamilton, Routh, Jacobi, Liouville and Poincaré, who studied the extent to which one can reduce the dimension of the phase space of the system by making use of any available symmetries and associated conservation laws. Corresponding to the main two views of mechanics, namely Hamiltonian and Lagrangian mechanics, one can also adopt two views of reduction theory.

In symplectic and Poisson reduction, which are now well developed and much studied subjects, one focuses on how to pass the symplectic two form and the Poisson bracket structure as well as any associated Hamiltonian dynamics to a quotient space for the action of a symmetry group (see, for example, Meyer (1973), Marsden and Weinstein (1974), Marsden and Ratiu (1986) and the expositions in Abraham and Marsden (1978), Arnold (1989), Libermann and Marle (1987) and Marsden (1992)).

In Lagrangian reduction theory, which proceeds in a logically independent way, one emphasizes how the variational structure passes to a quotient space (see, for example, Cendra and Marsden (1987), Cendra, Ibort and Marsden (1987), Marsden and Scheurle [1993a, 1993b], Bloch, Krishnaprasad, Marsden and Ratiu (1996), Cendra, Holm, Marsden and Ratiu (1998), Holm, Marsden and Ratiu (1998a), Jalnapurkar and Marsden (2000) and Marsden, Ratiu and Scheurle (2000)). Of course, the two methodologies are related by the Legendre transform, although not always in a straightforward way.

The main purpose of this work is to further the development of Lagrangian reduction theory. There are several aspects to this program. First, we provide a context that allows for repeated Lagrangian reduction by the action of a symmetry group. Second, we provide the geometry that is useful for the expression of the reduced 
equations, called the Lagrange-Poincaré equations. Further details concerning the main results of this work are given shortly.

\subsection{Background}

As we have mentioned, in the last few years there has been considerable activity in the area of Lagrangian reduction in which one focuses on the reduction of variational principles. We shall review the background for this theory briefly, starting with the best known classical results.

\section{Classical Cases.}

Several classical instances of Lagrangian reduction are well known, such as Routh reduction which was developed by Routh (1877) in connection with his studies of the stability of relative equilibria. Routh began the development of what we would call today Lagrangian reduction for Abelian groups. One thinks of this case as treating mechanical systems with cyclic variables.

Another fundamental case is that of Euler-Poincaré reduction, which occurs for the case in which the configuration space is a Lie group. One thinks of this case as primarily intended for systems governed by Euler equations, such as those of a rigid body and a fluid. This case has its origins in the work of Lagrange (1788) and Poincaré (1901a). Both of them clearly had some idea of the reduction process.

In these classical works, many important ideas were developed. However, for both of these cases, some of the clarifications and generalizations are remarkably recent. For example, only in Bretherton (1970) was the reduced variational principle established for the fluid equations, but this was done by ad hoc rather than general methods. Both the intrinsic (coordinate free) formulation of Routh reduction as well as the general formulation of Euler-Poincaré reduction in terms of variational principles were given in Marsden and Scheurle [1993a, 1993b]). The Euler-Poincaré case was further developed in Bloch, Krishnaprasad, Marsden and Ratiu (1996). An exposition of Lagrangian reduction for both the Routh and Euler-Poincaré cases can be found in Marsden and Ratiu (1999).

\section{Semidirect Product Theory.}

Another well developed subject in Hamiltonian reduction theory is that of semidirect product theory. This theory has its origins in the work of Guillemin and Sternberg (1980) (see also Guillemin and Sternberg (1984)), Ratiu [1980a; 1981; 1982a], and Marsden, Ratiu, and Weinstein [1984a, 1984b]. This theory has many interesting applications, such as to the heavy top, MHD, and the dynamics of underwater vehicles (Leonard and Marsden (1997)). This semidirect product theory was a direct precursor to the development of symplectic reduction by stages (Marsden, Misiolek, Perlmutter and Ratiu $[1998,2000])$. 
Lagrangian analogues of the semidirect product theory were developed in Holm, Marsden and Ratiu (1998a) with applications to many fluid mechanical problems of interest. The point of view was to extend the Euler-Poincaré theory to the case of systems such as the heavy top and compressible flows in which there are advected parameters or fields. This methodology was applied to the case of the Maxwell-Vlasov equations by Cendra, Holm, Hoyle and Marsden (1998); Cendra, Holm, Marsden and Ratiu (1998) showed how it fits into the general framework of Lagrangian reduction.

\section{Symplectic versus Poisson Reduction.}

We should emphasize that in the framework of the theory of Poisson manifolds, Poisson reduction (in the naive sense of just taking nonsingular quotients, not the more sophisticated sense of Marsden and Ratiu (1986)) by stages is quite simple, while in the framework of symplectic manifolds, symplectic reduction by stages is more sophisticated. On the other hand, if one wants to study the reduction of cotangent bundles and to retain as much of this structure as possible, then even the Poisson point of view is quite nontrivial.

The Lagrangian analogue of symplectic reduction is nonabelian Routh reduction (Marsden and Scheurle [1993a, 1993b], Jalnapurkar and Marsden (2000) and Marsden, Ratiu and Scheurle (2000)) and its full development in the context of reduction by stages is the subject of a future publication. The present work represents a Lagrangian analogue of the Poisson version of reduction by stages but keeping the structure of the tangent bundle as much as possible. One of the things that makes the Lagrangian side interesting has been the lack of a general category that is the Lagrangian analogue of Poisson manifolds. Such a category, that of LagrangePoincaré bundles is given in $\S 8$, with the tangent bundle of a configuration manifold and a Lie algebra as its two most basic examples. We also develop the Lagrangian analogue of reduction for central extensions and, as in the case of symplectic reduction by stages, cocycles and curvatures enter in this context in a natural way.

\section{Nonholonomic Mechanics.}

The ideas of geometric mechanics and Lagrangian reduction have had a significant impact on the theory of nonholonomic systems (such as mechanical systems with rolling constraints), as in Bloch, Krishnaprasad, Marsden and Murray (1996) and Koon and Marsden [1997b, c, 1998], Marsden, Ratiu, and Weinstein [1998, 1994] Bloch and Crouch (1999) and Lewis [1996, 2000]. These references also develop Lagrangian reduction methods in the context of nonholonomic mechanics with symmetry. These methods have been quite useful in many control problems and in robotics. The techniques of the present paper can be used to give an intrinsic geometric meaning to the reduction of the Lagrange-d'Alembert equations

of nonholonomic mechanics. This is the subject of the work Cendra, Marsden and Ratiu (2000). 


\section{Control Theory.}

Geometric mechanics and Lagrangian reduction theory has also had a significant impact on control theory, including stabilization (as in Bloch, Leonard and Marsden (2000) and Jalnapurkar and Marsden (1999) as well as on optimal control theory; see Vershik and Gershkovich [1988, 1994], Bloch and Crouch [1993, 1994, 1995] Montgomery [1990, 1993], Koon and Marsden (1997a) and references therein.

\subsection{The Main Results of This Paper.}

We now give a few more details concerning the main results. The first of these, given in $\S 5$, develops the theory of Lagrange-Poincaré bundles, which enable one to perform Lagrangian reduction in stages. Lagrange-Poincaré bundles may be regarded as the Lagrangian analogue of a Poisson manifold in symplectic geometry. Lagrange-Poincaré bundles include, of course, the case of reduced tangent bundles $(T Q) / G$ in which we take the quotient of the tangent bundle of the configuration space $Q$ by the action of a Lie group $G$ on $Q$. This in turn, includes important examples such as Euler-Poincaré reduction for the special case $Q=G$, a Lie group, in which case, $(T Q) / G=\mathfrak{g}$, the Lie algebra of $G$. Euler-Poincaré reduction is now a textbook topic that can be found in Marsden and Ratiu (1999). We show that when a general tangent bundle is reduced by a group action, one ends up in the category of Lagrange-Poincaré bundles.

We mention that Lagrange-Poincaré bundles are in particular, Lie algebroids but carry additional structure. We will not use any theory of groupoids or algebroids in this work, but we will comment on part of the literature in the body of the work.

The Lagrange-Poincaré equations are expressed using connections and curvature. These equations are obtained using the idea of reducing variational principles. The Lagrange-Poincaré category is stable under reduction and the structure carried by Lagrange-Poincaré bundles is exactly what is needed to write the LagrangePoincaré equations in a covariant form.

In $\S 5.3$ and $\S 5.4$ we show that if the symmetry group has a normal subgroup (i.e., one has a group extension), then reducing by the whole group is shown to be isomorphic to what one gets by reducing in stages, first by the normal subgroup followed by reduction by the quotient group. This result is a Lagrangian analogue of doing Poisson reduction by stages, but keeping track of the local structure of Poisson manifolds, as in the Lie-Weinstein theorem (see Weinstein (1983a)).

The theory we establish may be viewed as the Lagrangian analogue of the bundle picture on the Hamiltonian side developed by Montgomery (1986) and Montgomery, Marsden and Ratiu (1984). This bundle picture was in turn influenced by work on Wong's equations for a particle in a Yang-Mills field, as studied by Sternberg (1977), Weinstein (1978), Montgomery (1984), and Koon and Marsden (1997a). As we shall see in $\S 3.3$, this theory has a very beautiful Lagrangian analogue. In $\S 6$ we give a number of additional examples. In future works we plan to establish additional links with the Hamiltonian side. 


\subsection{Future Work and Related Issues.}

Our theory naturally suggests a number of additional things that warrant further investigation.

\section{Geometric Phases.}

The development of the theory of geometric phases in the Lagrangian context is natural to develop given the relatively large amount of activity from the symplectic and Poisson point of view (see, eg, Marsden, Montgomery and Ratiu (1990), Marsden (1992), Blaom (2000) and references therein).

The paper of Marsden, Ratiu and Scheurle (2000) gives geometric phase formulas in the context of Routh reduction. The development of geometric phases by stages would be of interest. In fact, the Lagrangian setting provides natural connections and also a natural setting for averaging which is one of the basic ingredients in geometric phases.

\section{Nonholonomic Mechanics.}

As mentioned above, the work of Cendra, Marsden and Ratiu (2000) extends the notion of Lagrange-Poincaré bundles to those of Lagrange-d'Alembert-Poincaré bundles that are appropriate for nonholonomic mechanics. This extension may be regarded as the Lagrangian analogue of the notion of an almost Poisson manifold (in which Jacobi's identity can fail), as in Koon and Marsden (1998) and Cannas da Silva and Weinstein (1999). Furthering the links with almost Poisson manifolds and also developing a nonholonomic reduction by stages theory would of course be of interest.

\section{Further Relations with the Hamiltonian Side.}

It would also be significant to investigate the precise relationship of the work here with the Hamiltonian reduction by stages theory in more detail, in particular, the relation with symplectic reduction by stages applied to cotangent bundles. This requires the nonabelian Routh reduction analogue of the work here, namely that of Marsden, Ratiu and Scheurle (2000), which extends the work of Marsden and Scheurle (1993a) and Jalnapurkar and Marsden (2000).

\section{Relations with Poisson Geometry.}

The Lie-Weinstein theorem states that a Poisson manifold is locally the product of a symplectic manifold and the dual of a Lie algebra. This paper develops a Lagrangian category that locally looks like the dual of this local structure for Poisson manifolds. Our bundles actually have more structure than this, which is important for carrying out the reduction, namely we also carry along a connection and a 
two-form that helps keep track of curvature (or magnetic terms). This structure is also very useful for writing covariant versions of the reduced equations, that is, the Lagrange-Poincaré equations.

\section{Variational Integrators and Discrete Reduction.}

As Weinstein (1996) points out, there is a more general context for Lagrangian mechanics that also includes discrete mechanics in the sense of Veselov [1988, 1991] and Moser and Veselov (1991). Our category of Lagrange-Poincaré bundles does not include this literally, but still, there is a well defined discrete analogue of these bundles. This picture is useful in the understanding of the reduced Hamilton-Jacobi equation (see Ge and Marsden (1988)).

One of the interesting developments in symplectic integration algorithms has been the progress made in variational integrators. These are based on direct discretizations of Hamilton's principle following some of the ideas of Veselov (1988). See, for example, Wendlandt and Marsden (1997), Marsden, Patrick and Shkoller (1998) and Kane et al. (2000). There is a very interesting discrete reduction theory for these that is still under development. See Marsden, Pekarsky and Shkoller (1999), Bobenko and Suris [1999a, 1999b] and Jalnapurkar Jalnapurkar, Leok, Marsden and West (2000).

\section{Infinite Dimensional Examples.}

In this paper we will be dealing with Lagrangian reduction theory in the context of finite dimensional manifolds. Of course the theory formally applies to many interesting infinite dimensional examples. In the infinite dimensional context, many of the expressions that appear here as pure partial derivatives must be written in the notation of functional derivatives (see Marsden and Ratiu (1999) for some of the basic examples, an explanation of the functional derivative notation, and additional references to the literature).

\section{Multisymplectic Context.}

Another area of much current activity is that of multisymplectic geometry. See, for example, Marsden and Shkoller (1999) and Marsden, Patrick and Shkoller (1998). This theory has both a Lagrangian and a Hamiltonian view and it has allowed, for example, a development of the Moser-Veselov theory to the context of PDE's. Reduction theory in this context is in its infancy (see, for example, Marsden, Montgomery, Morrison and Thompson (1986) and Castrillón-López, Ratiu and Shkoller (2000)). Obviously it would be of interest to develop such a theory from the Lagrangian reduction point of view.

An exciting possible application where reduction by stages is involved is that of various complex fluids, such as liquid crystals, where there is a group of particle relabeling symmetries, as in fluids and plasmas, as well as an internal order param- 
eter group. See Holm (2000). This sort of example would also provide an interesting context for multisymplectic reduction by stages! 
1. Introduction 
2

\section{Preliminary Constructions}

In this section we recall some results about bundles and connections that we will use later. As a general reference, see Kobayashi and Nomizu (1963); however, the reader should be warned that various conventions and notations differ from this reference and we shall point these out as we proceed. In general, we follow the conventions of Abraham and Marsden (1978) and Abraham, Marsden and Ratiu (1988). We provide intrinsic proofs with a view to infinite dimensional generalizations and also because of the insight they provide. Coordinate expressions are important in many applications, and they can be readily provided (some of these may be found in Marsden and Scheurle [1993a, 1993b] and Bloch, Krishnaprasad, Marsden and Murray (1996)).

\subsection{Notation and general assumptions.}

Maps. If $f: A \rightarrow B$ is a map between sets and $C \subset A$, we shall often write $f: C \rightarrow B$ instead of $f \mid C: C \rightarrow B$, for short.

\section{Manifolds.}

Unless otherwise noted, for simplicity, manifolds are assumed to be $C^{\infty}$, as are maps between them. While the manifolds will be assumed to be finite dimensional, many results can be easily generalized for infinite dimensions in a straightforward manner. 
For a manifold $Q$, we let $T Q$ be the tangent bundle of $Q$. An element of $T_{q} Q$ will be denoted $v_{q}, u_{q}, \ldots$, or (using a standard abuse of notation) by $(q, \dot{q})$ or simply by $\dot{q}$.

\section{Actions.}

Unless otherwise noted, an action $\rho: G \times Q \rightarrow Q$ of a Lie group $G$ on a manifold $Q$ is assumed to satisfy the additional condition that, relative to this action, the manifold $Q$ becomes a principal bundle with structure group $G$, say $\pi_{G}(Q): Q \rightarrow Q / G$. In other words, we assume that $Q / G$ is a manifold, that $\pi_{G}(Q)$ is a submersion and that the action is free. As is well known (see, for example, Abraham and Marsden (1978) for the proof), if the action is both free and proper, then this hypothesis is satisfied.

We will often use the equivalent notations $\pi_{G}(Q)(q)=[q]_{G}$ for the equivalence class of $q \in Q$. We will work with left actions in this paper unless explicitly noted otherwise; however, generalizations for right actions are straightforward.

The assumption of freeness of the action is, of course, a strong one and it corresponds, in the Hamiltonian case, to eliminating the case of singular reduction (see Sjamaar and Lerman (1991), Bates and Lerman (1997), Ortega (1998), and Ortega and Ratiu [1997, 2001]). Similarly, on the Lagrangian side, this paper does not address the case of singular reduction. Of course, such questions are very interesting, but there is plenty to do even omitting that topic.

We will often use the equivalent notations $\rho(g, q) \equiv \rho_{g}(q) \equiv \rho_{q}(g) \equiv g \cdot q \equiv g q$ for the action of the group element $g$ on the point $q \in Q$. However, the concatenation notation $g q$ will be used most commonly. The tangent lift of this action will be denoted $g v_{q}$, where $v_{q} \in T_{q} Q$. For any element $\xi$ in the Lie algebra $\mathfrak{g}$ of $G$, the infinitesimal generator at $q \in Q$ is denoted $\xi q \equiv \xi_{Q}(q)$ and it is defined, as usual, by

$$
\xi q:=\left.\frac{d}{d t}\right|_{t=0}(\exp t \xi) q .
$$

\section{Bundles.}

If $\pi: P \rightarrow Q$ is a fiber bundle and $q \in Q$, the fiber $\pi^{-1}(q)$ at $q$ is sometimes denoted $P_{q}$. If $\tau_{i}: V_{i} \rightarrow Q_{i}$ are vector bundles for $i=1,2$ and $f: V_{1} \rightarrow V_{2}$ is a vector bundle map, the induced map on the zero-sections, which are usually identified with $Q_{i}$, $i=1,2$, is denoted $f_{0}: Q_{1} \rightarrow Q_{2}$, or sometimes, by a slight abuse of notation, simply $f$.

Given two bundles $\pi_{i}: P_{i} \rightarrow Q, i=1,2$, the fiber product is the bundle $\pi_{1} \times{ }_{Q} \pi_{2}: P_{1} \times{ }_{Q} P_{2} \rightarrow Q$ where $P_{1} \times{ }_{Q} P_{2}$ is the set of all elements $\left(p_{1}, p_{2}\right) \in$ $P_{1} \times P_{2}$ such that $\pi_{1}\left(p_{1}\right)=\pi_{2}\left(p_{2}\right)$ and the projection $\pi_{1} \times{ }_{Q} \pi_{2}: P_{1} \times{ }_{Q} P_{2} \rightarrow Q$ is naturally defined by $\pi_{1} \times{ }_{Q} \pi_{2}\left(p_{1}, p_{2}\right)=\pi_{1}\left(p_{1}\right)=\pi_{2}\left(p_{2}\right)$. The fiber is given by $\left(\pi_{1} \times_{Q} \pi_{2}\right)^{-1}(q)=\pi_{1}^{-1}(q) \times \pi_{2}^{-1}(q)$.

A principal bundle is a manifold $Q$ with a free left action $G \times Q \rightarrow Q$ of a Lie group $G$, such that the natural projection $\pi: Q \rightarrow Q / G$ is a submersion. 
The Whitney sum of two vector bundles $\tau_{i}: V_{i} \rightarrow Q, i=1,2$, over the same base is their fiber product. It is a vector bundle over $Q$ and is denoted $V_{1} \oplus V_{2}$. This bundle is obtained by taking the fiberwise direct sum of the fibers of $V_{1}$ and $V_{2}$.

Sometimes, for purposes of uniformizing notation, we will consider a manifold $Q$ as being identified with the vector bundle over $Q$ whose fibers have dimension 0 . Therefore if $\tau: V \rightarrow Q$ is a vector bundle, $Q \oplus V$ is simply $V$, and an element $q \oplus v$ of $Q \oplus V$ satisfies $\tau v=q$.

\section{Connections.}

The word connection will be used in two different but standard senses in this paper. Sometimes it will mean a principal connection on a principal bundle and sometimes will mean a connection on a vector bundle, usually denoted $\nabla$ with the addition, sometimes, of some indexes to clarify the spaces on which the connection is defined. In any case, the context will always make it clear the sense in which we are using the word. We will recall some of the key notions and conventions used in the following paragraphs.

\subsection{Connections on Principal Bundles.}

Horizontal and Vertical Spaces. Let $\pi: Q \rightarrow Q / G$ be a left principal bundle, where $\pi$ is the canonical projection. Recall that a (principal) connection $A$ on $Q$ is a Lie algebra valued one form $A: T Q \rightarrow \mathfrak{g}$ with the properties

(i) $A(\xi q)=\xi$ for all $\xi \in \mathfrak{g}$; that is, $A$ takes infinitesimal generators of a given Lie algebra element to that element, and

(ii) $A\left(T_{q} \rho_{g} \cdot v\right)=\operatorname{Ad}_{g}(A(v))$, where $\operatorname{Ad}_{g}$ denotes the adjoint action of $G$ on $\mathfrak{g}$.

The restriction of a connection to the tangent space $T_{q} Q$ is denoted $A_{q}$. Recall that connections may be characterized by giving their vertical and horizontal spaces defined at $q \in Q$ by

$$
\operatorname{Ver}_{q}=\operatorname{Ker} T_{q} \pi, \quad \operatorname{Hor}_{q}=\operatorname{Ker} A_{q} .
$$

Thus, $\operatorname{Ver}(T Q)=\cup_{q \in Q} \operatorname{Ver}_{q}$ is the subbundle of vectors tangent to the group orbits. The vertical and horizontal components of a vector $v_{q}$ will be denoted $\operatorname{Ver}\left(v_{q}\right)$ and $\operatorname{Hor}\left(v_{q}\right)$ respectively. By definition,

$$
\operatorname{Ver}\left(v_{q}\right)=A\left(v_{q}\right) q \text { and } \operatorname{Hor}\left(v_{q}\right)=v_{q}-A\left(v_{q}\right) q .
$$

This provides a decomposition $T Q=\operatorname{Hor}(T Q) \oplus \operatorname{Ver}(T Q)$ where $\operatorname{Hor}(T Q)=$ $\cup_{q \in Q} \operatorname{Hor}_{q}$ and $\operatorname{Ver}(T Q)$ are the horizontal and vertical subbundles of $T Q$, which are invariant under the action of $G$. A vector is called horizontal if its vertical component is zero; i.e., if $A\left(v_{q}\right)=0$ and it is called vertical if its horizontal component is zero; i.e., if $T_{q} \pi\left(v_{q}\right)=0$. Note that $T_{q} \pi: \operatorname{Hor}_{q} \rightarrow T_{\pi(q)}(Q / G)$ is an isomorphism. 


\section{Curvature.}

The curvature of $A$ will be denoted $B^{A}$ or simply $B$. By definition, it is the Lie algebra valued two form on $Q$ defined by

$$
B\left(u_{q}, v_{q}\right)=\mathbf{d} A\left(\operatorname{Hor}_{q}\left(u_{q}\right), \operatorname{Hor}_{q}\left(v_{q}\right)\right),
$$

where $\mathbf{d}$ denotes the exterior derivative.

The reader should be aware that, although the notion of the curvature of a given connection on a principal bundle is a well established and an essentially unique concept, there are many conventions related to various sign conventions and definitions of the wedge product and the exterior derivative. The one adopted here for left actions is consistent with the one given in Abraham and Marsden (1978).

\section{Cartan Structure Equations.}

The Cartan structure equations state that for vector fields $u, v$ (not necessarily horizontal) on $Q$, we have

$$
B(u, v)=\mathbf{d} A(u, v)-[A(u), A(v)],
$$

where the bracket on the right hand side is the Lie bracket in $\mathfrak{g}$. We write this equation for short as

$$
B=\mathbf{d} A-[A, A]
$$

\section{Horizontal Lifts.}

Given a vector $X \in T_{x}(Q / G)$, and $q \in \pi^{-1}(x)$, the horizontal lift $X_{q}^{h}$ of $X$ at $q$ is the unique horizontal vector in $T_{q} Q$ that projects via $T \pi$ to the vector $X(x)$; that is, $X_{q}^{h} \in\left(T_{q} \pi\right)^{-1}(X)$. We denote by $X^{h}$ the vector field along $\pi^{-1}(x)$ formed by all horizontal lifts of $X$ at points of $\pi^{-1}(x)$.

For any curve $x(t)$ in $Q / G$, where $t \in[a, b]$, the family of horizontal lifts is denoted $x^{h}$. The definition is the following. For any point $q_{0} \in \pi^{-1}\left(x_{0}\right)$, where $x_{0}=x\left(t_{0}\right)$, for some $t_{0} \in[a, b]$, the horizontal lift of $x(t)$, which at $t=t_{0}$ coincides with $q_{0}$, is uniquely determined by requiring its tangent to be a horizontal vector. This curve is denoted $x_{q_{0}}^{h}$ and is defined on $[a, b]$.

Consider a curve $q(t)$, where $t \in[a, b]$, and choose $t_{0} \in[a, b]$. Then there is a unique horizontal curve $q_{h}(t)$ such that $q_{h}\left(t_{0}\right)=q\left(t_{0}\right)$ and $\pi\left(q_{h}(t)\right)=\pi(q(t))$ for all $t \in[a, b]$. Therefore, we can define a curve $g_{q}(t), t \in[a, b]$ in $G$ by the decomposition

$$
q(t)=g_{q}(t) q_{h}(t)
$$

for all $t \in[a, b]$. Evidently $g_{q}\left(t_{0}\right)$ is the identity. Also, notice that if $x(t)=\pi(q(t))$ and $q_{0}=q\left(t_{0}\right)$ then $q_{h}(t)=x_{q_{0}}^{h}(t)$.

Lemma 2.2.1. For any curve $q(t), t \in[a, b]$ in $Q$ we have

$$
A(q, \dot{q})=\dot{g}_{q} g_{q}^{-1} \text {. }
$$


Proof. We start with the equality $g_{q}(t) q_{h}(t)=q(t)$. Differentiating this with respect to $t$ gives

$$
\dot{g}_{q}(t) q_{h}(t)+g_{q}(t) \dot{q}_{h}(t)=\dot{q}(t) .
$$

Here, the notation is interpreted in an obvious way; for example, for $u_{g} \in T_{g} G$ and $q \in Q, u_{g} q$ means the derivative of the orbit map $g \mapsto g q$ in the direction of $u_{g}$ to give an element of $T_{g q} Q$.

By definition of a horizontal vector, $A\left(g_{q}(t) \dot{q}_{h}(t)\right)=0$. Recall also that the connection reproduces the Lie algebra element on infinitesimal generators (i.e., $A(\xi q)=\xi$ for $\xi \in \mathfrak{g}$ and $q \in Q)$. In particular, for $\xi=\dot{g}_{q} g_{q}^{-1}$ and $q(t)=g_{q}(t) q_{h}(t)$ we get

$$
A\left(\dot{g}_{q}(t) q_{h}(t)\right)=A\left(\dot{g}_{q}(t) g_{q}^{-1}(t) g_{q}(t) q_{h}(t)\right)=\dot{g}_{q} g_{q}^{-1},
$$

from which the result follows.

Curvature and Horizontal Lifts. For $X_{1}, X_{2} \in \mathfrak{X}^{\infty}(Q / G)$, let $X_{1}^{h}, X_{2}^{h} \in \mathfrak{X}^{\infty}(Q)$ be their horizontal lifts. Thus, $X_{i}^{h}$ and $X_{i}$ are $\pi$-related, that is, $T \pi \circ X_{i}^{h}=X_{i} \circ \pi$, for $i=1,2$. However, the bracket operation of vector fields preserves $\pi$-relatedness (see, for example, Abraham, Marsden and Ratiu (1988)) and hence $T \pi \circ\left[X_{1}^{h}, X_{2}^{h}\right]=$ $\left[X_{1}, X_{2}\right] \circ \pi$. Thus, Hor $\left[X_{1}^{h}, X_{2}^{h}\right](q)$ and $\left[X_{1}, X_{2}\right]^{h}(q)$ are two horizontal vectors that project by $T_{q} \pi$ to $\left[X_{1}, X_{2}\right](\pi(q))$ and hence they are equal. This proves the identity

$$
\operatorname{Hor}\left[X_{1}^{h}, X_{2}^{h}\right]=\left[X_{1}, X_{2}\right]^{h} \text {. }
$$

By Cartan's structure equations, we get

$$
\begin{aligned}
B(q)\left(X_{1}(q), X_{2}(q)\right) q & =\left(\mathbf{d} A(q)\left(X_{1}(q), X_{2}(q)\right)\right) q \\
& =-\left(A\left(\left[X_{1}^{h}, X_{2}^{h}\right]\right)(q)\right) q \\
& =\operatorname{Ver}\left[X_{1}^{h}, X_{2}^{h}\right](q) \\
& =-\left[X_{1}^{h}, X_{2}^{h}\right]+\operatorname{Hor}\left[X_{1}^{h}, X_{2}^{h}\right](q),
\end{aligned}
$$

which shows, using (2.2.3), that

$$
\left[X_{1}^{h}, X_{2}^{h}\right](q)=\left[X_{1}, X_{2}\right]^{h}(q)-B(q)\left(X_{1}^{h}(q), X_{2}^{h}(q)\right) q .
$$

\subsection{Associated Bundles.}

Besides the principal bundle $\pi: Q \rightarrow Q / G$ discussed above, consider a left action $\rho: G \times M \rightarrow M$ of the Lie group $G$ on a manifold $M$. The associated bundle with standard fiber $M$ is, by definition,

$$
Q \times{ }_{G} M=(Q \times M) / G,
$$

where the action of $G$ on $Q \times M$ is given by $g(q, m)=(g q, g m)$. The class (or orbit) of $(q, m)$ is denoted $[q, m]_{G}$ or simply $[q, m]$. The projection $\pi_{M}: Q \times_{G} M \rightarrow Q / G$ is defined by $\pi_{M}\left([q, m]_{G}\right)=\pi(q)$ and it is easy to check that it is well defined and is a surjective submersion. 


\section{Parallel Transport.}

Let $\left[q_{0}, m_{0}\right]_{G} \in Q \times_{G} M$ and let $x_{0}=\pi\left(q_{0}\right) \in Q / G$. Let $x(t), t \in[a, b]$ be a curve in $Q / G$ and let $t_{0} \in[a, b]$ be such that $x\left(t_{0}\right)=x_{0}$. The parallel transport of this element $\left[q_{0}, m_{0}\right]_{G}$ along the curve $x(t)$ is defined to be the curve

$$
[q, m]_{G}(t)=\left[x_{q_{0}}^{h}(t), m_{0}\right]_{G} .
$$

Let us check that this curve is well defined. In fact, for any $g \in G$, the equivariance property of the connection gives $x_{g q_{0}}^{h}(t)=g x_{q_{0}}^{h}(t)$ for all $t$ and hence

$$
\left[x_{g q_{0}}^{h}(t), g m_{0}\right]_{G}=\left[g x_{q_{0}}^{h}(t), g m_{0}\right]_{G}=\left[x_{q_{0}}^{h}(t), m_{0}\right]_{G}
$$

for all $t$.

Consider a curve $x(t), t \in[a, b]$ in $Q / G$, as before. For $t, t+s \in[a, b]$, we adopt the notation

$$
\tau_{t+s}^{t}: \pi_{M}^{-1}(x(t)) \rightarrow \pi_{M}^{-1}(x(t+s))
$$

for the parallel transport map along the curve $x(s)$ of any point

$$
[q(t), m(t)]_{G} \in \pi_{M}^{-1}(x(t))
$$

to the corresponding point

$$
\tau_{t+s}^{t}[q(t), m(t)]_{G} \in \pi_{M}^{-1}(x(t+s)) .
$$

Thus,

$$
\tau_{t+s}^{t}[q(t), m(t)]_{G}=\left[x_{q(t)}^{h}(t+s), m(t)\right]_{G}
$$

\section{Associated Vector Bundles.}

Now we concentrate on the particular case when $M$ is a vector space and $\rho$ is a linear representation. (These will be the only associated bundles needed in the present work.) In this case, the associated bundle with standard fiber $M$ is a vector bundle in a natural way. We now recall what the vector bundle structure is. If $[q, m]_{G},\left[q, m_{1}\right]_{G},\left[q, m_{2}\right]_{G} \in \pi_{M}^{-1}\left([q]_{G}\right)$, then the vector space structure in this fiber is defined by

$$
a[q, m]_{G}=[q, a m]_{G} \quad \text { and } \quad\left[q, m_{1}\right]_{G}+\left[q, m_{2}\right]_{G}=\left[q, m_{1}+m_{2}\right]_{G} .
$$

We shall sometimes use the notation $\rho^{\prime}(\xi)$ for the second component of the infinitesimal generator of an element $\xi \in \mathfrak{g}$, that is, $\xi m=\left(m, \rho^{\prime}(\xi) m\right)$. Here we are using the identification $T M=M \times M$, appropriate for vector spaces. Thus, we are thinking of the infinitesimal generator as a map $\rho^{\prime}: \mathfrak{g} \rightarrow \operatorname{End}(M)$ (the linear vector fields on $M$ are identified with the space of linear maps of $M$ to itself). Thus, we have a linear representation of the Lie algebra $\mathfrak{g}$ on the vector space $M$. 
Definition 2.3.1. Let $[q(t), m(t)]_{G}, t \in[a, b]$ be a curve in $Q \times_{G} M$, denote by

$$
x(t)=\pi_{M}\left([q(t), m(t)]_{G}\right)=\pi(q(t))
$$

its projection on the base $Q / G$, and let, as above, $\tau_{t+s}^{t}$, where $t, t+s \in[a, b]$, denote parallel transport along $x(t)$ from time $t$ to time $t+s$. The covariant derivative of $[q(t), m(t)]_{G}$ along $x(t)$ is defined as follows

$$
\frac{D[q(t), m(t)]_{G}}{D t}=\lim _{s \rightarrow 0} \frac{\tau_{t}^{t+s}\left([q(t+s), m(t+s)]_{G}\right)-[q(t), m(t)]_{G}}{s} .
$$

Thus, the covariant derivative of $[q(t), m(t)]_{G}$ is an element of $\pi_{M}^{-1}(x(t))$.

Notice that if $[q(t), m(t)]_{G}$ is a vertical curve, then its base point is constant; that is, for each $t \in[a, b]$,

$$
x(t+s)=\pi_{M}\left([q(t+s), m(t+s)]_{G}\right)=x(t),
$$

so that $x_{q(t)}^{h}(t+s)=q(t)$ for all $s$. Therefore,

$$
\tau_{t}^{t+s}[q(t+s), m(t+s)]_{G}=\left[x_{q(t+s)}^{h}(t), m(t+s)\right]_{G}=[q(t), m(t+s)]_{G}
$$

and so we get the well known fact that the covariant derivative of a vertical curve in the associated bundle is just the fiber derivative. That is,

$$
\frac{D[q(t), m(t)]_{G}}{D t}=\left[q(t), m^{\prime}(t)\right]_{G}
$$

where $m^{\prime}(t)$ is the time derivative of $m$.

\section{Affine Connections.}

The notion of covariant derivative can be defined from a different, more axiomatic, point of view, which will be useful later in this paper. By definition (see Kobayashi and Nomizu (1963)), a connection (sometimes called an affine connection to distinguish it from a principal connection) $\nabla$ on a vector bundle $\tau: V \rightarrow Q$ is a map $\nabla: \mathfrak{X}^{\infty}(Q) \times \Gamma(V) \rightarrow \Gamma(V)$, say $(X, v) \mapsto \nabla_{X} v$, having the following two properties:

1. First, we require that

$$
\nabla_{f_{1} X_{1}+f_{2} X_{2}} v=f_{1} \nabla_{X_{1}} v+f_{2} \nabla_{X_{2}} v
$$

for all $X_{i} \in \mathfrak{X}^{\infty}(Q)$ (the space of smooth vector fields on $Q$ ), $f_{i} \in C^{\infty}(Q)$ (the space of smooth real valued functions on $Q$ ), $i=1,2$, and all $v \in \Gamma(V)$ (the space of smooth sections of the vector bundle $V$ ),

2. and secondly,

$$
\nabla_{X}\left(f_{1} v_{1}+f_{2} v_{2}\right)=X\left[f_{1}\right] v_{1}+f_{1} \nabla_{X} v_{1}+X\left[f_{2}\right] v_{2}+f_{2} \nabla_{X} v_{2}
$$

for all $X \in \mathfrak{X}^{\infty}(Q), f_{i} \in C^{\infty}(Q)$, and $v_{i} \in \Gamma(V), i=1,2$. 
Here, $X[f]$ denotes the derivative of $f$ in the direction of the vector field $X$.

Given a connection on $V$, the parallel transport of a vector $v_{0} \in \tau^{-1}\left(q_{0}\right)$ along a curve $q(t)$ in $Q, t \in[a, b]$ such that $q\left(t_{0}\right)=q_{0}$ for a fixed $t_{0} \in[a, b]$, is the unique curve $v(t)$ such that $v(t) \in \tau^{-1}(q(t))$ for all $t, v\left(t_{0}\right)=v_{0}$, and which satisfies $\nabla_{\dot{q}(t)} v(t)=0$ for all $t$. The operation of parallel transport establishes, as before, for each $t, s \in[a, b]$, a linear map

$$
T_{t+s}^{t}: \tau^{-1}(q(t)) \rightarrow \tau^{-1}(q(t+s))
$$

associated to each curve $q(t)$ in $Q$. Then we can define the operation of covariant derivative on curves $v(t)$ in $V$ similar to that in the previous definition; that is,

$$
\frac{D v(t)}{D t}=\left.\frac{d}{d s} T_{t}^{t+s} v(t+s)\right|_{s=0} .
$$

Observe that the connection $\nabla$ can be recovered from the covariant derivative (and thus from the parallel transport operation). Indeed, $\nabla$ is given by

$$
\nabla_{X} v\left(q_{0}\right)=\left.\frac{D}{D t} v(t)\right|_{t=t_{0}},
$$

where, for each $q_{0} \in Q$, each $X \in \mathfrak{X}^{\infty}(Q)$, and each $v \in \Gamma(V)$, we have, by definition, that $q(t)$ is any curve in $Q$ such that $\dot{q}\left(t_{0}\right)=X\left(q_{0}\right)$ and $v(t)=v(q(t))$ for all $t$. This property establishes, in particular, the uniqueness of the connection associated to the covariant derivative $D / D t$.

\section{Formula for the Covariant Derivative Induced by a Principal Connection.}

Now we return to the study of the affine connection induced on an associated bundle. The following formula gives the relation between the covariant derivative of the affine connection and the principal connection.

\section{Lemma 2.3.2.}

$$
\frac{D[q(t), m(t)]_{G}}{D t}=\left[q(t),-\rho^{\prime}(A(q(t), \dot{q}(t))) m(t)+\dot{m}(t)\right]_{G} .
$$

Proof. For fixed $t$ and for any $s$ we have

$$
[q(t+s), m(t+s)]_{G}=\left[g_{q}(t+s) q_{h}(t+s), m(t+s)\right]_{G},
$$

where we assume $q_{h}(t)=q(t)$ and $g_{q}(t)=e$, the identity element of $G$. Then

$$
\begin{aligned}
\tau_{t}^{t+s}[q(t+s), m(t+s)]_{G} & =\tau_{t}^{t+s}\left[g_{q}(t+s) q_{h}(t+s), m(t+s)\right]_{G} \\
& =\tau_{t}^{t+s}\left[q_{h}(t+s), g_{q}(t+s)^{-1} m(t+s)\right]_{G} \\
& =\left[q_{h}(t), g_{q}(t+s)^{-1} m(t+s)\right]_{G} .
\end{aligned}
$$


Therefore, differentiating with respect to $s$ at $s=0$ we obtain

$$
\frac{D[q(t), m(t)]_{G}}{D t}=\left[q(t),-\dot{g}_{q}(t) m(t)+\dot{m}(t)\right]_{G}
$$

Since $g_{q}(t)=e$ we have, using Lemma 2.2.1, that $\dot{g}_{q}(t)=A(q(t), \dot{q}(t))$. Therefore,

$$
\dot{g}_{q}(t) m(t)=\rho^{\prime}(A(q(t), \dot{q}(t))) m(t) .
$$

\section{Induced Connections on Associated Bundles.}

The previous definition of the covariant derivative of a curve in the associated vector bundle $Q \times{ }_{G} M$ thus leads to a connection on $Q \times_{G} M$. Let us call this connection $\tilde{\nabla}^{A}$ or simply $\tilde{\nabla}$.

We now describe this connection $\tilde{\nabla}^{A}$ in more detail. Let $\varphi: Q / G \rightarrow Q \times_{G} M$ be a section of the associated bundle and let $X(x) \in T_{x}(Q / G)$ be a given vector tangent to $Q / G$ at $x$. Let $x(t)$ be a curve in $Q / G$ such that $\dot{x}(0)=X(x)$; thus, $\varphi(x(t))$ is a curve in $Q \times_{G} M$. The covariant derivative of the section $\varphi$ with respect to $X$ at $x$ is then, by definition,

$$
\tilde{\nabla}_{X(x)}^{A} \varphi=\left.\frac{D \varphi(x(t))}{D t}\right|_{t=0}
$$

Notice that we only need to know $\varphi$ along the curve $x(t)$ in order to calculate the covariant derivative.

The notion of a horizontal curve $[q(t), m(t)]_{G}$ on $Q \times_{G} M$ is defined by the condition that its covariant derivative vanishes. A vector tangent to $Q \times_{G} M$ is called horizontal if it is tangent to a horizontal curve. Correspondingly, the horizontal space at a point $[q, m]_{G} \in Q \times_{G} M$ is the space of all horizontal vectors at $[q, m]_{G}$.

\section{The Adjoint Bundle.}

The case that interests us most in this paper occurs when $M=\mathfrak{g}$ and $\rho_{g}$ is the adjoint action $\operatorname{Ad}_{g}$.

Definition 2.3.3. The associated bundle with standard fiber $\mathfrak{g}$, where the action of $G$ on $\mathfrak{g}$ is the adjoint action, is called the adjoint bundle, and is sometimes denoted $\operatorname{Ad}(Q)$. We will use the notation $\tilde{\mathfrak{g}}:=\operatorname{Ad}(Q)$ in this paper. We let $\tilde{\pi}_{G}: \tilde{\mathfrak{g}} \rightarrow Q / G$ denote the projection given by $\tilde{\pi}_{G}\left([q, \xi]_{G}\right)=[q]_{G}$.

Lemma 2.3.4. Let $[q(s), \xi(s)]_{G}$ be any curve in $\tilde{\mathfrak{g}}$. Then

$$
\frac{D[q(s), \xi(s)]_{G}}{D s}=[q(s),-[A(q(s), \dot{q}(s)), \xi(s)]+\dot{\xi}(s)]_{G} .
$$

Proof. Use the previous lemma and the fact that $\rho^{\prime}(\xi)=\operatorname{ad}_{\xi}$.

The next Lemma says that the adjoint bundle is a Lie algebra bundle. 
Lemma 2.3.5. Each fiber $\tilde{\mathfrak{g}}_{x}$ of $\tilde{\mathfrak{g}}$ carries a natural Lie algebra structure defined by

$$
\left[[q, \xi]_{G},[q, \eta]_{G}\right]=[q,[\xi, \eta]]_{G} .
$$

Proof. We must show that the bracket is well defined, which is done in a straightforward way as follows:

$$
\begin{aligned}
{\left[\left[g q, \operatorname{Ad}_{g} \xi\right]_{G},\left[g q, \operatorname{Ad}_{g} \eta\right]_{G}\right] } & =\left[g q,\left[\operatorname{Ad}_{g} \xi, \operatorname{Ad}_{g} \eta\right]\right]_{G} \\
& =\left[g q, \operatorname{Ad}_{g}[\xi, \eta]\right]_{G} \\
& =[q,[\xi, \eta]]_{G} \\
& =\left[[q, \xi]_{G},[q, \eta]_{G}\right] .
\end{aligned}
$$

\subsection{The Bundles $T Q / G$ and $T(Q / G) \oplus \tilde{\mathfrak{g}}$}

Let $\pi: Q \rightarrow Q / G$ be a principal bundle with structure group $G$, as before. The tangent lift of the action of $G$ on $Q$ defines an action of $G$ on $T Q$ and so we can form the quotient $(T Q) / G=: T Q / G$. There is a well defined map $\tau_{Q} / G: T Q / G \rightarrow$ $Q / G$ induced by the tangent of the projection map $\pi: Q \rightarrow Q / G$ and given by $\left[v_{q}\right]_{G} \mapsto[q]_{Q}$. The vector bundle structure of $T Q$ is inherited by this bundle.

Lemma 2.4.1. The rules

$$
\left[v_{q}\right]_{G}+\left[u_{q}\right]_{G}=\left[v_{q}+u_{q}\right]_{G} \text { and } \lambda\left[v_{q}\right]_{G}=\left[\lambda v_{q}\right]_{G},
$$

where $\lambda \in \mathbb{R}, v_{q}, u_{q} \in T_{q} Q$, and $\left[v_{q}\right]_{G}$ and $\left[u_{q}\right]_{G}$ are their equivalence classes in the quotient $T Q / G$, define a vector bundle structure on $T Q / G$ having base $Q / G$. The fiber $(T Q / G)_{x}$ is isomorphic, as a vector space, to $T_{q} Q$, for each $x=[q]_{G}$.

Proof. If $\left[q_{0}\right]_{G}=x$ is given, the isomorphism between the fiber $(T Q / G)_{x}$ and $T_{q_{0}} Q$ is given by the map $\left[u_{q}\right]_{G} \mapsto g^{-1} u_{q}$, where $g \in G$ is uniquely determined by the relation $g q_{0}=q$.

The bundle $T Q / G$ is a fundamental object in the present paper. One can state reduced variational principles in a natural way in terms of this bundle without any reference to a connection on $Q$, which we shall describe in the next section.

It is, however, also interesting to introduce an (arbitrarily chosen) connection on $Q$ relative to which one can realize the space $T Q / G$ in a convenient way as well as writing the Lagrange-Poincaré equations in an interesting form. The next lemma is one of the main tools needed for doing this.

Lemma 2.4.2. The map $\alpha_{A}: T Q / G \rightarrow T(Q / G) \oplus \tilde{\mathfrak{g}}$ defined by

$$
\alpha_{A}\left([q, \dot{q}]_{G}\right)=T \pi(q, \dot{q}) \oplus[q, A(q, \dot{q})]_{G}
$$

is a well defined vector bundle isomorphism. The inverse of $\alpha_{A}$ is given by

$$
\alpha_{A}^{-1}\left((x, \dot{x}) \oplus[q, \xi]_{G}\right)=\left[(x, \dot{x})_{q}^{h}+\xi q\right]_{G} .
$$


Proof. To show that $\alpha_{A}$ is well defined, observe that for any $g \in G$ we have $T \pi(g q, g \dot{q})=T \pi(q, \dot{q})$ and also

$$
[g q, A(g q, g \dot{q})]_{G}=\left[g q, \operatorname{Ad}_{g} A(q, \dot{q})\right]_{G}=[q, A(q, \dot{q})]_{G} .
$$

Then we see that

$$
\alpha_{A}\left([g q, g \dot{q}]_{G}\right)=\alpha_{A}\left([q, \dot{q}]_{G}\right) .
$$

To show that $\alpha_{A}^{-1}$ is well defined, notice that $(x, \dot{x})_{g q}^{h}=g(x, \dot{x})_{q}^{h}$ and that $\left(\operatorname{Ad}_{g} \xi\right) g q=$ $g \xi q$. Therefore,

$$
\alpha_{A}^{-1}\left((x, \dot{x}) \oplus\left[g q, \operatorname{Ad}_{g} \xi\right]_{G}\right)=\alpha_{A}^{-1}\left((x, \dot{x}) \oplus[q, \xi]_{G}\right) .
$$

\section{Remark.}

The bundles $T Q / G$ and $T(Q / G) \oplus \tilde{\mathfrak{g}}$ do not depend on the connection $A$, but, of course, $\alpha_{A}$ does. 


\section{3}

\section{The Lagrange-Poincaré Equations}

In this section we use the constructions from the previous section to show how to write the Lagrange-Poincaré equations in an intrinsic way. This is done in terms of an (arbitrarily chosen) connection $A$. The resulting equations are given on the bundle $T^{(2)}(Q / G) \oplus \tilde{\mathfrak{g}}$, where $T^{(2)}(Q / G)$ is the second order tangent bundle (which is related to the 2-jet bundle) of $Q / G$, whose general definition is recalled in $\S 3.2$, and where $\tilde{\mathfrak{g}}=Q \times_{G} \mathfrak{g}$ is the associated adjoint bundle to $Q$. A key point in doing this is to decompose arbitrary variations of curves in $Q$ into vertical and horizontal components. This gives rise, correspondingly, to two reduced equations, namely, vertical Lagrange-Poincaré equations, corresponding to vertical variations, and horizontal Lagrange-Poincaré equations, corresponding to horizontal variations, which are Euler-Lagrange equations on $Q / G$ with an additional term involving the curvature $B$ of $A$. (Our conventions for the curvature were given in $\S 2.2$ )

\subsection{The Geometry of Variations}

\section{Spaces of Curves.}

The Setup. Fix a time interval $I=\left[t_{0}, t_{1}\right]$. The space of all (smooth) curves from $I$ to $Q$ will be denoted $\Omega(Q)$. We shall not include the interval $I$ explicitly in the notation for spaces of curves for simplicity; it will be understood from the context and explicitly stated when necessary.

Given a map $f: Q_{1} \rightarrow Q_{2}$, the map $\Omega(f): \Omega\left(Q_{1}\right) \rightarrow \Omega\left(Q_{2}\right)$ is defined by

$$
\Omega(f)(q)(t)=f(q(t)),
$$


for $q(t)$ an element of $\Omega\left(Q_{1}\right)$. For given $q_{i} \in Q, i=0,1$, by definition, $\Omega\left(Q ; q_{0}\right)$ and $\Omega\left(Q ; q_{0}, q_{1}\right)$ are, respectively, the spaces of curves $q(t)$ on $Q$ such that $q\left(t_{0}\right)=q_{0}$ and $q\left(t_{i}\right)=q_{i}, i=0,1$.

If $\pi: Q \rightarrow S$ is a bundle, $q_{0} \in Q$ and $\pi\left(q_{0}\right)=x_{0}$, then $\Omega\left(Q ; x_{0}\right)$ denotes the space of all curves in $\Omega(Q)$ such that $\pi\left(q\left(t_{0}\right)\right)=x_{0}$. The space $\Omega\left(Q ; x_{1}\right)$ is defined in an analogous way. Similarly, $\Omega\left(Q ; x_{0}, q_{1}\right)$ is the space of all curves in $\Omega(Q)$ such that $\pi\left(q\left(t_{0}\right)\right)=x_{0}$ and $q\left(t_{1}\right)=q_{1}$. The spaces of curves $\Omega\left(Q ; q_{0}, x_{1}\right), \Omega\left(Q ; x_{0}, x_{1}\right)$, etc. are defined in a similar way.

If $V \rightarrow Q$ and $W \rightarrow Q$ are vector bundles then $\Omega(V) \rightarrow \Omega(Q)$ and $\Omega(W) \rightarrow$ $\Omega(Q)$ are vector bundles in a natural way and there is a natural identification $\Omega(V \oplus W) \equiv \Omega(V) \oplus \Omega(W)$.

\section{Deformations of Curves.}

A deformation of a curve $q(t)$ on a manifold $Q$ is, by definition, a (smooth) function $q(t, \lambda)$ such that $q(t, 0)=q(t)$ for all $t$. The corresponding variation is defined by

$$
\delta q(t)=\left.\frac{\partial q(t, \lambda)}{\partial \lambda}\right|_{\lambda=0} .
$$

Variations of curves $q(t)$ belonging to $\Omega\left(Q ; q_{0}\right)$ or $\Omega\left(Q ; q_{0}, q_{1}\right)$ satisfy the corresponding fixed endpoints conditions, namely, $\delta q\left(t_{0}\right)=0$ or $\delta q\left(t_{i}\right)=0$ for $i=0,1$, respectively.

Let $\tau: V \rightarrow Q$ be a vector bundle and let $v(t, \lambda)$ be a deformation in $V$ of a curve $v(t)$ in $V$. If $\tau(v(t, \lambda))=q(t)$ does not depend on $\lambda$ we will call $v(t, \lambda)$ a $V$-fiber deformation of $v(t)$, or simply, a fiber deformation of $v(t)$. For each $t$, the variation

$$
\delta v(t)=\left.\frac{\partial v(t, \lambda)}{\partial \lambda}\right|_{\lambda=0}
$$

may be naturally identified with an element, also called $\delta v(t)$, of $\tau^{-1}(q(t))$. In this case, the curve $\delta v$ in $V$ is, by definition, a $V$-fiber variation of the curve $v$, or, simply, a fiber variation of the curve $v$.

\section{Horizontal and Vertical Variations.}

We now break up the variation of a curve into horizontal and vertical parts. Thus, we consider a curve $q \in \Omega\left(Q ; q_{0}\right)$ (again, it is understood that the curves are defined on a fixed time interval $\left.\left[t_{0}, t_{1}\right]\right)$, where, as before, $Q \rightarrow Q / G$ is a principal bundle with a connection $A$.

A vertical variation $\delta q$ of $q$ satisfies, by definition, the condition $\delta q(t)=$ $\operatorname{Ver}(\delta q(t))$ for all $t$. Similarly, a horizontal variation satisfies $\delta q(t)=\operatorname{Hor}(\delta q(t))$ for all $t$.

Clearly, any variation $\delta q$ can be uniquely decomposed as follows:

$$
\delta q(t)=\operatorname{Hor}(\delta q(t))+\operatorname{Ver}(\delta q(t))
$$


for all $t$, where $\operatorname{Ver}(\delta q(t))=A(q(t), \delta q(t)) q(t)$ and where $\operatorname{Hor}(\delta q(t))=\delta q(t)-$ $\operatorname{Ver}(\delta q(t))$.

\section{Structure of Vertical Variations.}

Given a curve $q \in \Omega\left(Q ; q_{0}, q_{1}\right)$, let $v=A(q, \dot{q}) \in \mathfrak{g}$. Variations $\delta q$ of $q(t)$ induce corresponding variations $\delta v \in \mathfrak{g}$ in the obvious way:

$$
\delta v=\left.\frac{\partial A(q(t, \lambda), \dot{q}(t, \lambda))}{\partial \lambda}\right|_{\lambda=0} .
$$

Consider the decomposition $q=g_{q} q_{h}$ introduced in equation (2.2.2). A vertical deformation $q(t, \lambda)$ can be written as $q(t, \lambda)=g_{q}(t, \lambda) q_{h}(t)$. The corresponding variation $\delta q(t)=\delta g_{q}(t) q_{h}(t)$ is of course also vertical.

Now we introduce some important notation. Define the curve

$$
\eta(t)=\delta g_{q}(t) g_{q}(t)^{-1}
$$

in $\mathfrak{g}$. The fixed endpoint condition gives $\eta\left(t_{i}\right)=0, i=1,2$.

Notice that, by construction,

$$
\delta q(t)=\delta g_{q}(t) q_{h}(t)=\eta(t) g_{q}(t) q_{h}(t)=\eta(t) q(t) .
$$

Lemma 3.1.1. For any vertical variation $\delta q=\eta q$ of a curve $q \in \Omega\left(Q ; q_{0}, q_{1}\right)$ the corresponding variation $\delta v$ of $v=A(q, \dot{q})$ is given by $\delta v=\dot{\eta}+[\eta, v]$ with $\eta_{i}=0$, $i=0,1$.

Proof. We will give the proof in the case that $G$ is a matrix group. The more general case can be treated using the appendix to Bloch, Krishnaprasad, Marsden and Ratiu (1996).

By Lemma 2.2.1, we have $v=\dot{g}_{q} g_{q}^{-1}$. Then

$$
\begin{aligned}
\delta v & =\left(\delta \dot{g}_{q}\right) g_{q}^{-1}-\dot{g}_{q} g_{q}^{-1} \delta g_{q} g_{q}^{-1} \\
& =\left(\delta g_{q}\right) g_{q}^{-1}-v \eta \\
& =\left(\dot{\eta} g_{q}+\eta \dot{g}_{q}\right) g_{q}^{-1}-v \eta \\
& =\dot{\eta}+[\eta, v] .
\end{aligned}
$$

\section{Note.}

In the variational approach to the Euler-Poincaré equations (see Marsden and Ratiu (1999), Chapter 13), there is a class of constrained variations $\delta \xi=\dot{\eta}+$ $[\xi, \eta]$ introduced for computing the corresponding variational principle. The above construction of $v, \eta$ is not computing the same objects. These constrained variations 
are, instead, special instances of the construction of covariant variations, to be introduced shortly in Definition 3.1.3. In the second remark following Lemma 3.1.4, we shall explicitly remark on how the constructions of variations for the EulerPoincaré equations and those for the Lagrange-Poincaré case are related.

\section{The Structure of Horizontal Variations.}

Now we calculate variations $\delta v$ corresponding to horizontal variations $\delta q$ of a curve $q \in \Omega\left(Q ; q_{0}, q_{1}\right)$.

Lemma 3.1.2. Let $\delta q$ be a horizontal variation of a curve $q \in \Omega\left(Q ; q_{0}, q_{1}\right)$. Then the corresponding variation $\delta v$ of $v=A(q, \dot{q})$ satisfies $\delta v=B(q)(\delta q, \dot{q})$.

Proof. Let $q(t, \lambda)$ be a horizontal deformation of $q(t)$, that is, $\lambda \mapsto q(t, \lambda)$ is a horizontal curve for each $t$. Now we work locally in a local trivialization of the bundle and write the connection $A$ in the following way:

$$
v=A(q, \dot{q})=\langle\mathcal{A}(q), \dot{q}\rangle
$$

Then, we compute using the chain rule:

$$
\delta v=\left.\frac{\partial v}{\partial \lambda}\right|_{\lambda=0}=\left.\left\langle D \mathcal{A}(q) \cdot \frac{\partial q}{\partial \lambda}, \dot{q}\right\rangle\right|_{\lambda=0}+\left.\left\langle\mathcal{A}(q), \frac{\partial^{2} q}{\partial \lambda \partial t}\right\rangle\right|_{\lambda=0} .
$$

On the other hand, since $\lambda \mapsto q(t, \lambda)$ is horizontal,

$$
\left\langle\mathcal{A}(q), \frac{\partial q}{\partial \lambda}\right\rangle=0
$$

and so, by differentiating with respect to $t$,

$$
0=\left.\left\langle D \mathcal{A}(q) \cdot \dot{q}, \frac{\partial q}{\partial \lambda}\right\rangle\right|_{\lambda=0}+\left.\left\langle\mathcal{A}(q), \frac{\partial^{2} q}{\partial t \partial \lambda}\right\rangle\right|_{\lambda=0}
$$

Then we obtain, by subtraction,

$$
\delta v=\left.\mathbf{d} \mathcal{A}(q)\left(\frac{\partial q}{\partial \lambda}, \frac{\partial q}{\partial t}\right)\right|_{\lambda=0}
$$

Since $\partial q / \partial \lambda$ is horizontal, Cartan's structure equation (2.2.1) implies

$$
\delta v=\left.B(q)\left(\frac{\partial q}{\partial \lambda}, \frac{\partial q}{\partial t}\right)\right|_{\lambda=0}
$$

or, in other words, $\delta v=B(q)(\delta q, \dot{q})$. 


\section{The Covariant Variation on the Adjoint Bundle.}

Any curve in $Q, q \in \Omega\left(Q ; q_{0}, q_{1}\right)$ induces a curve in $\tilde{\mathfrak{g}}$ in a natural way, namely,

$$
[q, v]_{G}(t)=[q(t), v(t)]_{G},
$$

where $v(t)=A(q, \dot{q})$. Observe that, for each $t,[q, v]_{G}(t) \in \tilde{\mathfrak{g}}_{x(t)}$ (the fiber over $\left.x(t)\right)$, where $x(t)=\pi(q(t))$ for all $t$. We want to study variations $\delta[q, v]_{G}$ corresponding to vertical and also to horizontal variations $\delta q$ of $q$.

While vertical variations $\delta q$ give rise to vertical variations $\delta[q, v]_{G}$, horizontal variations $\delta q$ need not give rise to horizontal variations $\delta[q, v]_{G}$. The deviation of any variation $\delta[q, v]_{G}$ from being horizontal is measured by the covariant variation $\delta^{A}[q, v]_{G}(t)$, defined as follows

Definition 3.1.3. For any given deformation $q(t, \lambda)$ of $q(t)$, the covariant variation $\delta^{A}[q, v]_{G}(t)$ is defined by

$$
\delta^{A}[q, v]_{G}(t)=\left.\frac{D[q(t, \lambda), v(t, \lambda)]_{G}}{D \lambda}\right|_{\lambda=0} .
$$

\section{Vertical Variations and the Adjoint Bundle.}

We first consider the case of vertical variations.

Lemma 3.1.4. The covariant variation $\delta^{A}[q, v]_{G}(t)$ corresponding to a vertical variation $\delta q=\eta q$ is given by

$$
\delta^{A}[q, v]_{G}(t)=\frac{D[q, \eta]_{G}}{D t}+[q,[v, \eta]]_{G} .
$$

Proof. Let $q(t, \lambda)$ be a vertical deformation of $q(t)$ with, as usual, $\delta q=\partial q /\left.\partial \lambda\right|_{\lambda=0}$. As we saw before, $q(t, \lambda)=g_{q}(t, \lambda) q_{h}(t)$ and $\delta g_{q}=\eta g_{q}$, where $\eta=\delta g_{q} g_{q}^{-1}=$ $A(q, \delta q)$. If we let $v=A(q, \dot{q})$, by Lemma 3.1.1, we have $\delta v=\dot{\eta}+[\eta, v]$. From this and Lemma 2.3.4, we obtain

$$
\begin{aligned}
\delta^{A}[q, v]_{G}(t) & =[q,-[A(q, \delta q), v]+\delta v]_{G} \\
& =[q,-[\eta, v]+\dot{\eta}+[\eta, v]]_{G} \\
& =[q, \dot{\eta}]_{G} .
\end{aligned}
$$

Again, by Lemma 2.3.4, we have

$$
\begin{aligned}
\frac{D[q, \eta]_{G}}{D t} & =[q,-[A(q, \dot{q}), \eta]+\dot{\eta}]_{G} \\
& =[q,-[v, \eta]+\dot{\eta}]_{G} \\
& =[q, \dot{\eta}+[\eta, v]]_{G} .
\end{aligned}
$$

Therefore,

$$
\delta^{A}[q, v]_{G}(t)=\frac{D[q, \eta]_{G}}{D t}+[q,[v, \eta]]_{G}
$$




\section{Remarks.}

1. In view of Lemma 2.3.5, we can write

$$
[q,[v, \eta]]_{G}=\left[[q, v]_{G},[q, \eta]_{G}\right] .
$$

2. Let us now show that the formula $\delta v=\dot{\eta}+[v, \eta]$ for the constrained variations for the Euler-Poincaré equations (see Marsden and Ratiu (1999) and references therein) coincides with the construction of the covariant variation given in Definition 3.1.3. Given a Lie group $G$, we regard it as a principal bundle over a point; that is, we take $G=Q$. The identification of $\mathfrak{g}$ with $T(Q / G) \oplus \tilde{\mathfrak{g}}$ in this case is given by $v \mapsto[e, v]_{G}$. Then this equivalence defines $\delta v \equiv \delta^{A}[e, v]_{G}$ and then the preceding lemma shows that $\delta v=\dot{\eta}+[v, \eta]$, which is the same type of variation one has for the Euler-Poincaré equations.

\section{The Reduced Curvature Form.}

In preparation for the consideration of variations $\delta^{A}[q, v]_{G}(t)$ corresponding to horizontal variations, we prove the following result of independent interest.

Lemma 3.1.5. The curvature 2-form $B \equiv B^{A}$ of the connection $A$ induces a $\tilde{\mathfrak{g}}$-valued 2 -form $\tilde{B} \equiv \tilde{B}^{A}$ on $Q / G$ given by

$$
\tilde{B}(x)(\delta x, \dot{x})=[q, B(q)(\delta q, \dot{q})]_{G},
$$

where for each $(x, \dot{x})$ and $(x, \delta x)$ in $T_{x}(Q / G),(q, \dot{q})$ and $(q, \delta q)$ are any elements of $T_{q} Q$ such that $\pi(q)=x, T \pi(q, \dot{q})=(x, \dot{x})$ and $T \pi(q, \delta q)=(x, \delta x)$.

Proof. We show that the right hand side does not depend on the choice of $(q, \dot{q})$ and $(q, \delta q)$. For any $g \in G$ we have

$$
\begin{aligned}
{[g q, B(g q)(g \delta q, g \dot{q})]_{G} } & =\left[g q, \operatorname{Ad}_{g} B(q)(\delta q, \dot{q})\right]_{G} \\
& =[q, B(q)(\delta q, \dot{q})]_{G} .
\end{aligned}
$$

Definition 3.1.6. The $\tilde{\mathfrak{g}}$-valued 2 -form $\tilde{B}$ on $Q / G$ will be called the reduced curvature form.

\section{Horizontal Variations and the Adjoint Bundle.}

Now we are ready to describe covariant variations $\delta^{A}[q, v]_{G}(t)$ corresponding to horizontal variations $\delta q$.

Lemma 3.1.7. Variations $\delta^{A}[q, v]_{G}(t)$ corresponding to horizontal variations $\delta q$ are given by

$$
\delta^{A}[q, v]_{G}(t)=\tilde{B}(x)(\delta x, \dot{x})(t),
$$

where $T \pi(q, \dot{q})=(x, \dot{x}), T \pi(q, \delta q)=(x, \delta x)$, and $v=A(q, \dot{q})$. 
Proof. By Lemma 2.3.4, we have $\delta^{A}[q, v]_{G}(t)=[q,-[A(q, \delta q), v]+\delta v]_{G}$. Since $\delta q$ is horizontal, we have $A(q, \delta q)=0$. Using this and lemmas 3.1.2 and 3.1.5, we obtain $\delta^{A}[q, v]_{G}(t)=\tilde{B}(x)(\delta x, \dot{x})$.

\subsection{The Euler-Lagrange and Euler-Poincaré Operators}

The purpose of the next three sections is to carry out the reduction of the EulerLagrange equations by means of reduction of Hamilton's principle using the geometric set up in the preceding section. We will begin in this section with some geometric preliminaries and treat the standard case of the Euler-Lagrange and the Euler-Poincaré equations.

\section{Reduced Spaces of Curves.}

In what follows we shall often identify the bundles $T Q / G$ and $T(Q / G) \oplus \tilde{\mathfrak{g}}$, using the isomorphism $\alpha_{A}$ of Lemma 2.4.2. This leads to other natural identifications as well. For instance, the reduced set of curves $\left[\Omega\left(Q ; q_{0}, q_{1}\right)\right]_{G}$ is the set of curves $[q]_{G}(t)=[q(t)]_{G}$ on $Q / G$ such that the curve $q(t)$ belongs to $\Omega\left(Q ; q_{0}, q_{1}\right)$. This reduced set of curves is naturally identified with the set of curves $[q(t), \dot{q}(t)]_{G}$ in $T Q / G$ such that $q\left(t_{i}\right)=q_{i}$, for $i=0,1$, and in turn, this is identified, via the map

$$
\Omega\left(\alpha_{A}\right):\left[\Omega\left(Q ; q_{0}, q_{1}\right)\right]_{G} \rightarrow \Omega(T(Q / G) \oplus \tilde{\mathfrak{g}}),
$$

with the set of curves

$$
T \pi(q(t), \dot{q}(t)) \oplus[q(t), A(q(t), \dot{q}(t))]_{G}
$$

in $T(Q / G) \oplus \tilde{\mathfrak{g}}$, such that $q\left(t_{i}\right)=q_{i}$, for $i=0,1$. The image of this reduced set of curves will be denoted $\Omega\left(\alpha_{A}\right)\left(\left[\Omega\left(Q ; q_{0}, q_{1}\right)\right]_{G}\right)$.

\section{The Reduced Lagrangian.}

Let $L: T Q \rightarrow \mathbb{R}$ be an invariant Lagrangian, that is, $L(g(q, \dot{q}))=L(q, \dot{q})$ for all $(q, \dot{q}) \in T Q$ and all $g \in G$. Because of this invariance, we get a well defined reduced Lagrangian $l: T Q / G \rightarrow \mathbb{R}$ satisfying

$$
l\left([q, \dot{q}]_{G}\right)=L(q, \dot{q}) .
$$

As we will see in detail in this section, the evolution of the reduced system will be a critical point, say a curve $[q]_{G}$ in the reduced set of curves $\left[\Omega\left(Q ; q_{0}, q_{1}\right)\right]_{G}$, of the reduced action

$$
\int_{t_{0}}^{t_{1}} l\left([q, \dot{q}]_{G}\right) d t
$$


for suitable types of variations.

However, variations of curves in the reduced family of curves are not of the usual sort found in Hamilton's principle, and so the equations of motion in the bundle $T Q / G$ cannot be written in a direct way.

In this section we use the description of vertical and horizontal variations given in the preceding section to derive equations of motion in the bundle $T^{(2)}(Q / G) \oplus 2 \tilde{\mathfrak{g}}$ defined below. Equations corresponding to vertical variations will be called the vertical Lagrange-Poincaré equations, and equations corresponding to horizontal variations will be called the horizontal Lagrange-Poincaré equations.

\section{Identification of Bundles.}

We shall allow a slight abuse of notation, namely we will consider $l$ as a function defined on $T(Q / G) \oplus \tilde{\mathfrak{g}}$ or $T Q / G$ interchangeably, using the isomorphism $\alpha_{A}$. Also we shall often use a slight abuse of the variable-notation for a function, namely we will write $l(x, \dot{x}, \bar{v})$ to emphasize the dependence of $l$ on $(x, \dot{x}) \in T(Q / G)$ and $\bar{v} \in \tilde{\mathfrak{g}}$. However one should keep in mind that $x, \dot{x}$ and $\bar{v}$ cannot be considered as being independent variables unless $\tilde{\mathfrak{g}}$ and $T(Q / G)$ are trivial bundles. Even in those cases in which $\tilde{\mathfrak{g}}$ and $T(Q / G)$ are trivial, and therefore $x, \dot{x}$ and $\bar{v}$ can be considered as being independent variables in a natural way, it is sometimes convenient to proceed using the general theory.

\section{The $k$ th Order Tangent Bundle.}

We define below the $k$ th-order tangent bundle $\tau_{Q}^{(k)}: T^{(k)} Q \rightarrow Q$. For $\bar{q} \in Q$, elements of $T_{\bar{q}}^{(k)} Q$ are equivalence classes of curves in $Q$, namely, two given curves $q_{i}(t), i=1,2$, such that $q_{1}\left(\bar{t}_{1}\right)=q_{2}\left(\bar{t}_{2}\right)=\bar{q}$ are equivalent, by definition, if and only if in any local chart we have $q_{1}^{(l)}\left(\bar{t}_{1}\right)=q_{2}^{(l)}\left(\bar{t}_{2}\right)$, for $l=1,2, \ldots, k$, where $q^{(l)}$ denotes the derivative of order $l$. The equivalence class of the curve $q(t)$ at $\bar{q}=q(\bar{t})$ will be denoted $[q]_{\bar{q}}^{(k)}$. The projection

$$
\tau_{Q}^{(k)}: T^{(k)} Q \rightarrow Q \quad \text { is given by } \quad \tau_{Q}^{(k)}\left([q]_{\bar{q}}^{(k)}\right)=\bar{q} .
$$

It is clear that $T^{(0)} Q=Q, T^{(1)} Q=T Q$, and that, for $l<k$, there is a well defined fiber bundle structure

$$
\tau_{Q}^{(l, k)}: T^{(k)} Q \rightarrow T^{(l)} Q, \quad \text { given by } \quad \tau_{Q}^{(l, k)}\left([q]_{\bar{q}}^{(k)}\right)=[q]_{\bar{q}}^{(l)} .
$$

The bundles $T^{(k)} Q$ for $k>1$ are not vector bundles, except for $k=1$. The bundle $T^{(2)} Q$ is often denoted $\ddot{Q}$, and is called the second order bundle (see, for example, Marsden, Patrick and Shkoller (1998), Marsden and Ratiu (1999) and references therein). 


\section{Relation to Jet Bundles.}

Consider the bundle $\mathbb{R} \times Q \rightarrow \mathbb{R}$, whose sections are curves in $Q$ (the fields of classical mechanics). Then the $k$-jet bundle of this bundle may be identified with the bundle $\mathbb{R} \times T^{(k)} Q \rightarrow \mathbb{R} \times Q$, where the first component of this map is the identity.

From the point of view of jet bundles associated to maps between two manifolds, $T^{(k)} Q$ coincides with the fiber bundle $J_{0}^{k}(\mathbb{R}, Q)$ formed by $k$-jets of curves from $\mathbb{R}$ to $Q$ (based at $0 \in \mathbb{R}$ ), as defined, for example in Bourbaki (1983) or Kolár, Michor, and Slovák (1993).

\section{Properties of $k$ th Order Tangent Bundles.}

It is also easy to see that for any map $f: M \rightarrow N$ we have a naturally induced map

$$
T^{(k)} f: T^{(k)} M \rightarrow T^{(k)} N \quad \text { given by } \quad T^{(k)} f\left([q]_{\bar{q}}^{(k)}\right)=[f \circ q]_{f(\bar{q})}^{(k)} .
$$

In particular, a group action $\rho: G \times Q \rightarrow Q$ can be naturally lifted to a group action

$$
\rho^{(k)}: G \times T^{(k)} Q \rightarrow T^{(k)} Q \quad \text { given by } \quad \rho_{g}^{(k)}\left([q]_{\bar{q}}^{(k)}\right)=\left[\rho_{g} \circ q\right]_{\rho(g, \bar{q})}^{(k)} .
$$

We will often denote $\rho_{g}^{(k)}\left([q]_{\bar{q}}^{(k)}\right)=\rho^{(k)}\left(g,[q]_{\bar{q}}^{(k)}\right)=g[q]_{\bar{q}}^{(k)}$.

Let $M \times N$ be the Cartesian product of the manifolds $M$ and $N$. Then, for any $(\bar{m}, \bar{n}) \in M \times N$ there is a natural identification $T_{(\bar{m}, \bar{n})}^{(k)}\left((M \times N) \equiv T_{\bar{m}}^{(k)} M \times T_{\bar{n}}^{(k)} N\right.$, which induces an identification $T^{(k)}(M \times N) \equiv T^{(k)} M \times T^{(k)} N$.

The natural action of $G$ on the fiber bundle $T^{(k)} Q$ endows $T^{(k)} Q$ with a principal bundle structure with structure group $G$. The quotient $T^{(k)} Q / G$ can be easily shown to be a fiber bundle over the base $Q / G$. The bundle $T^{(2)} Q / G$ is the one that interests us most in this paper, because the Lagrange-Poincaré operator of a reduced Lagrangian is defined on $T^{(2)} Q / G$ with values in $T^{*}(Q / G) \oplus \tilde{\mathfrak{g}}^{*}$, as we shall see in $\S 3.3$. The class of the element $[q]_{\bar{q}}^{(k)}$ in the quotient $T^{(k)} Q / G$ will be denoted $\left[[q]_{\bar{q}}^{(k)}\right]_{G}$, as usual. Since we have the projection $\pi_{G}(Q): Q \rightarrow Q / G$ we obtain a bundle map

$$
T^{(k)} \pi_{G}(Q): T^{(k)} Q \rightarrow T^{(k)}(Q / G)
$$

Moreover, it can be easily shown that this bundle map induces a well defined bundle map

$$
T^{(k)} Q / G \rightarrow T^{(k)}(Q / G) \quad \text { given by } \quad\left[[q]_{\bar{q}}^{(k)}\right]_{G} \mapsto T^{(k)} \pi_{G}(Q)\left([q]_{\bar{q}}^{(k)}\right) .
$$

Let $\bar{q} \in Q$, denote $\pi(\bar{q})=[\bar{q}]_{G}=\bar{x}$, and let $[x]_{\bar{x}}^{(k)} \in T^{(k)}(Q / G)$ be given. Let $x(t)$ be any curve belonging to the class $[x]_{\bar{x}}^{(k)}$. Then there is a unique horizontal lift $x_{\bar{q}}^{h}$ of $x(t)$. We define the horizontal lift of $[x]_{\bar{x}}^{(k)}$ at $\bar{q}$ by

$$
[x]_{\bar{x}, \bar{q}}^{(k), h}:=\left[x_{\bar{q}}^{h}\right]_{\bar{q}}^{(k)} \text {. }
$$


We must also remark that $T^{(k)} G$ carries a natural Lie group structure. ${ }^{1}$ If $[g]_{\bar{g}}^{(k)}$, and $[h]_{\bar{h}}^{(k)}$ are classes of curves $g$ and $h$ in $G$, we define the product $[g]_{\bar{g}}^{(k)}[h]_{\bar{h}}^{(k)}$ as being the class $[g h]_{\bar{g} \bar{h}}^{(k)}$ at the point $\bar{g} \bar{h}$ of the curve $g h$. The Lie algebra $T_{e} T^{(k)} G$ of $T^{(k)} G$ can be naturally identified, as a vector space, with $(k+1) \mathfrak{g}$ (that is, the direct sum of $k+1$ copies of $\mathfrak{g}$ ), which, therefore, carries a unique Lie algebra structure such that this identification becomes a Lie algebra isomorphism. There is also a natural identification of $T_{e}^{(k)} G$ with $k \mathfrak{g}$.

Also, for $k=1,2, \ldots, T^{(k)} Q$ is a principal bundle with structure group $T^{(k)} G$ in a natural way. More precisely, if $[g]_{\bar{g}}^{(k)} \in T^{(k)} G$ is the class of a curve $g$ in $G$ and $[q]_{\bar{q}}^{(k)} \in T_{\bar{q}}^{(k)}(Q)$ is the class of a curve $q$ in $Q$ we let $[g]_{\bar{g}}^{(k)}[q]_{\bar{q}}^{(k)} \in T_{\bar{g} \bar{q}}^{(k)} Q$ denote the class $[g q]_{\bar{g}}^{(k)}$ of the curve $g q$ at the point $\bar{g} \bar{q}$. In particular, if $\xi \in k \mathfrak{g}$ and $[q]_{\bar{q}}^{(k)} \in T^{(k)} Q$ are given, there is a well defined element $\xi[q]_{\bar{q}}^{(k)} \in T^{(k)} Q$.

\section{Connection-like Structures on Higher Order Tangent Bundles.}

For $q \in \Omega(Q)$, we have the curve $[q(t), v(t)]_{G}$ in $\tilde{\mathfrak{g}}$, where $v(t)=A(q(t), \dot{q}(t))$. Lemma 2.3.4 shows that the covariant derivative of $[q(t), v(t)]_{G}$ is given by

$$
\begin{aligned}
\frac{D[q(t), v(t)]_{G}}{D t} & =[q(t),-[A(q(t), \dot{q}(t)), v(t)]+\dot{v}(t)]_{G} \\
& =[q(t), \dot{v}(t)]_{G} .
\end{aligned}
$$

The second covariant derivative of $[q(t), v(t)]_{G}$, again by Lemma 2.3.4, is given by

$$
\frac{D^{2}[q(t), v(t)]_{G}}{D t^{2}}=[q(t),-[v(t), \dot{v}(t)]+\ddot{v}(t)]_{G} .
$$

More generally, for each $k=1,2, \ldots$, we can find, by induction, a curve $v_{k}(t)$ in $\mathfrak{g}$, having an expression that involves $v(t)$ and the derivatives $v^{(l)}(t), l=1,2, \ldots, k-1$, such that

$$
\frac{D^{k-1}[q(t), v(t)]_{G}}{D t^{k-1}}=\left[q(t), v_{k}(t)\right]_{G} .
$$

More precisely, we have

$$
v_{1}=v \quad \text { and } \quad v_{k+1}=-\left[v, v_{k}\right]+\dot{v}_{k},
$$

\footnotetext{
${ }^{1}$ Recall that $T^{(1)} G=T G$ is the semidirect product group $G \subseteq \mathfrak{g}$ whose Lie algebra is the semidirect product $\mathfrak{g}(5) \mathfrak{g}$, where the second factor is regarded as the representation space of the adjoint action. This semidirect product Lie algebra is, as a vector space, equal to $2 \mathfrak{g}:=\mathfrak{g} \oplus \mathfrak{g}$. We will not need, or study, the Lie group structure of $T^{(k)} G$ in this paper, although this would be interesting to do. It would also be interesting to see if there is any relation between the structures here and the study of the algebras that occur in the BBGKY hierarchy, as in Marsden, Morrison and Weinstein (1984).
} 
for $k=1,2, \ldots$. In particular, we obtain

$$
v_{2}(t)=\dot{v}(t), \quad v_{3}(t)=-[v(t), \dot{v}(t)]+\ddot{v}(t),
$$

etc. In addition, we shall write, by definition, $v_{0}(t)=0$. Using the fact that $v(t)=$ $A(q(t), \dot{q}(t))$, we can also find expressions for $v_{k}(t)$ in coordinates in terms of $q^{(l)}(t)$, $l=1,2, \ldots, k$. We state the following lemma, which is readily proved.

Lemma 3.2.1. Let $q(t)$ be a given curve in $Q$ such that $q(\bar{t})=\bar{q}$. For each $k=1,2, \ldots$ the formula

$$
\bar{A}_{k}\left([q]_{\bar{q}}^{(k)}\right)=v_{k}(\bar{t})
$$

gives a well defined map $\bar{A}_{k}: T^{(k)} Q \rightarrow \mathfrak{g}$. Therefore there is also a well defined map $A_{k}: T^{(k)} Q \rightarrow k \mathfrak{g}$, given, for each $k=1,2, \ldots$, by

$$
A_{k}\left([q]_{\bar{q}}^{(k)}\right)=\oplus_{l=1}^{k} v_{l}(\bar{t})
$$

where we have written $k \mathfrak{g}$ to stand for the vector space direct sum $\oplus_{l=1}^{k} \mathfrak{g}$ of $k$ copies of $\mathfrak{g}$.

Let $g \in G$ and $[q]_{\bar{q}}^{(k)} \in T_{\bar{q}}^{(k)} Q$ be given. Then we can easily prove that

$$
A_{k}\left(g[q]_{\bar{q}}^{(k)}\right)=\operatorname{Ad}_{g} A_{k}\left([q]_{\bar{q}}^{(k)}\right),
$$

using induction, the definition of $A_{k}$, and taking into account the formulas

$$
\operatorname{Ad}_{g} \dot{v}_{k}=\frac{d}{d t} \operatorname{Ad}_{g} v_{k}
$$

and $\operatorname{Ad}_{g}\left[v, v_{k}\right]=\left[\operatorname{Ad}_{g} v, \operatorname{Ad}_{g} v_{k}\right]$. According to Lemma 2.2.1, for any curve $q(t)$ in $Q$ such that $q(\bar{t})=\bar{q}$ we have

$$
A(q, \dot{q})=\dot{g}_{q} g_{q}^{-1} .
$$

Using this equation and the definition of $v_{k}$, one can inductively find an expression for $v_{k}(\bar{t})$ in terms of $g_{q}^{(l)}(\bar{t})$, for $l=1,2, \ldots$ For instance, for the case of matrix groups, we can see directly that $v_{1}(\bar{t})=v(\bar{t})=\dot{g}_{q}(\bar{t}), v_{2}(\bar{t})=\ddot{g}_{q}(\bar{t})-\dot{g}_{q}(\bar{t})^{2}$, etc. It is not difficult to see that the expression for $v_{k}(\bar{t})$ is the sum of $g_{q}^{(k)}(\bar{t})$ plus terms involving only the lower order derivatives $g_{q}^{(l)}(\bar{t}), l=1,2, \ldots, k-1$. Using this fact, one sees that for any element $\xi=\left(\xi_{1}, \ldots \xi_{k}\right) \in k \mathfrak{g}$ and any element $\bar{q} \in Q$ there is a unique point $[q]_{\bar{q}}^{(k)} \in T_{\bar{q}}^{(k)} Q$ which equals $[g \bar{q}]_{\bar{q}}^{(k)}$ for a curve $g(t)$ in $G$ satisfying

$$
g(\bar{t})=e \quad \text { and } \quad A_{k}\left([q]_{\bar{q}}^{(k)}\right)=\xi .
$$

In fact, since we obviously have $g_{q}(t)=g(t)$, it is enough to find $g(t)$ such that the derivatives $g^{(l)}(\bar{t}), l=1,2, \ldots, k$, satisfy the appropriate conditions as explained 
above. We shall call this unique element $\xi \bar{q}$, as before, and the set of all such elements will be denoted $k \mathfrak{g} \bar{q}$. Moreover, it is not difficult to see that the restriction $A_{k}: k \mathfrak{g} \bar{q} \rightarrow k \mathfrak{g}$ is a diffeomorphism and therefore it naturally defines a unique vector space structure on $k \mathfrak{g} \bar{q}$ such that the restriction of $A_{k}$ becomes a linear isomorphism $A_{k}(\xi \bar{q})=\xi$, for all $x i \in k \mathfrak{g}$. By construction, we see that there is a natural identification between $k \mathfrak{g} \bar{q}$ and $T_{\bar{q}}^{(k)}(G \bar{q})$. Note that we have $A_{k}(\xi \bar{q})=\xi$ for all $\xi \in k \mathfrak{g}$, analogous to what one has for connections.

Let us define, for each $k=1,2, \ldots$, the vector bundle $k \tilde{\mathfrak{g}}$ as being the Whitney sum of $k$ copies of $\tilde{\mathfrak{g}}$. Define a map

$$
T^{(k)} Q \rightarrow k \tilde{\mathfrak{g}} \quad \text { by } \quad[q]_{\bar{q}}^{(k)} \mapsto\left[\bar{q}, A_{k}\left([q]_{\bar{q}}^{(k)}\right)\right]_{G},
$$

where the last term is defined by

$$
\left[\bar{q}, A_{k}\left([q]_{\bar{q}}^{(k)}\right)\right]_{G}=\oplus_{l=1}^{k}\left[\bar{q}, \bar{A}_{l}\left([q]_{\bar{q}}^{(l)}\right)\right]_{G} .
$$

The definitions show that, given any curve $q(t)$ in $Q$ such that $q(\bar{t})=\bar{q}$, we have, at $t=\bar{t}$,

$$
\left[\bar{q}, A_{k}\left([q]_{\bar{q}}^{(k)}\right)\right]_{G}=\left.\bigoplus_{l=1}^{k} \frac{D^{(l-1)}[q(t), v(t)]_{G}}{D t^{(l-1)}}\right|_{t=\bar{t}}
$$

We have essentially proven the following lemma, which generalizes Lemma 2.4.2

Lemma 3.2.2. The map

$$
\alpha_{A_{k}}: T^{(k)} Q / G \rightarrow T^{(k)}(Q / G) \times_{Q / G} k \tilde{\mathfrak{g}}
$$

defined by

$$
\alpha_{A_{k}}\left(\left[[q]_{\bar{q}}^{(k)}\right]_{G}\right)=T^{(k)} \pi_{G}(Q)\left([q]_{\bar{q}}^{(k)}\right) \times_{Q / G}\left[\bar{q}, A_{k}\left([q]_{\bar{q}}^{(k)}\right)\right]_{G}
$$

is a well defined bundle isomorphism. The inverse of $\alpha_{A_{k}}$ is given by

$$
\alpha_{A_{k}}^{-1}\left([x]_{\bar{x}}^{(k)} \times_{Q / G}[\bar{q}, \xi]_{G}\right)=\xi[x]_{\bar{x}, \bar{q}}^{(k), h} .
$$

Remark. As we have said earlier, the bundles $T^{(k)} Q$, for $k=1,2$, are the only ones that interest us in this paper. The cases $k=2,3, \ldots$ are needed, for instance, to deal with higher order Lagrangians $L: T^{(k)} Q \rightarrow \mathbb{R}$. Then the Euler-Lagrange operator $\mathcal{E} \mathcal{L}(L)$ will be defined on $T^{(k+1)} Q$ and will take values in a special vector bundle. There are also several interesting structures on the bundles $T^{(k)} Q$ which we will not study in the present paper. 


\section{Euler-Lagrange Operator.}

Next we introduce some notation and recall some basic results concerning EulerLagrange operators. The fundamental connection between the variational and differential-equation description of the evolution of a given system is given by the following well known result.

Theorem 3.2.3 (Euler-Lagrange). Let $L: T Q \rightarrow \mathbb{R}$ be a given Lagrangian on a manifold $Q$ and let

$$
\mathfrak{S}(L)(q)=\int_{t_{0}}^{t_{1}} L(q, \dot{q}) d t
$$

be the action of $L$ defined on $\Omega\left(Q ; q_{0}, q_{1}\right)$. Let $q(t, \lambda)$ be a deformation of a curve $q(t)$ in $\Omega\left(Q ; q_{0}, q_{1}\right)$ and let $\delta q(t)$ be the corresponding variation. Then, by definition, $\delta q\left(t_{i}\right)=0$ for $i=0,1$.

There is a unique bundle map

$$
\mathcal{E} \mathcal{L}(L): T^{(2)} Q \rightarrow T^{*} Q
$$

such that, for any deformation $q(t, \lambda)$, keeping the endpoints fixed, we have

$$
\mathbf{d} \mathfrak{S}(L)(q) \cdot \delta q=\int_{t_{0}}^{t_{1}} \mathcal{E} \mathcal{L}(L)(q, \dot{q}, \ddot{q}) \cdot \delta q
$$

where, as usual,

$$
\mathbf{d} \mathfrak{S}(L)(q) \cdot \delta q=\left.\frac{d}{d \lambda} \mathfrak{S}(L)(q(t, \lambda))\right|_{\lambda=0}
$$

with

$$
\delta q(t)=\left.\frac{\partial q(t, \lambda)}{\partial \lambda}\right|_{\lambda=0}
$$

The 1-form bundle-valued map $\mathcal{E} \mathcal{L}(L)$ is called the Euler-Lagrange operator.

In local coordinates $\mathcal{E} \mathcal{L}(L)$ has the following well known expression:

$$
\mathcal{E} \mathcal{L}(L)_{i}(q, \dot{q}, \ddot{q}) d q^{i}=\left(\frac{\partial L}{\partial q^{i}}(q, \dot{q})-\frac{d}{d t} \frac{\partial L}{\partial \dot{q}^{i}}(q, \dot{q})\right) d q^{i}
$$

in which it is understood that one regards the second term on the right hand side as a function on the second order tangent bundle by formally applying the chain rule and then replacing everywhere $d q / d t$ by $\dot{q}$ and $d \dot{q} / d t$ by $\ddot{q}$. The Euler-Lagrange equations can, of course, be written simply as $\mathcal{E} \mathcal{L}(L)(q, \dot{q}, \ddot{q})=0$.

\section{Euler-Poincaré Operator.}

Analogous to the Euler-Lagrange operator, the Euler-Poincaré theorem (Marsden and Scheurle (1993b) and Bloch, Krishnaprasad, Marsden and Ratiu (1996); see also Marsden and Ratiu (1999), section 13.5) induces an operator, called the EulerPoincaré operator. It is defined, as before, by the variational principle. 
Theorem 3.2.4 (Euler-Poincaré). Let $G$ be a Lie group, $L: T G \rightarrow \mathbb{R}$ a left $G$-invariant Lagrangian,

$$
\mathfrak{S}(L)(g)=\int_{t_{0}}^{t_{1}} L(g, \dot{g}) d t
$$

the action functional of $L$ defined on $\Omega\left(G ; g_{0}, g_{1}\right), l=L \mid \mathfrak{g}$ the reduced Lagrangian, and

$$
\mathfrak{S}_{\text {red }}(l)(v)=\int_{t_{0}}^{t_{1}} l(v) d t
$$

the reduced action functional defined on $\Omega(\mathfrak{g})$. Let $g(t, \lambda)$ be a deformation of a curve $g(t)$ in $\Omega\left(G ; g_{0}, g_{1}\right)$, keeping the endpoints fixed, and let $\delta g(t)$ be the corresponding variation; thus, by definition, $\delta g\left(t_{i}\right)=0$, for $i=0$, 1 . Let $v(t)=$ $g(t)^{-1} \dot{g}(t) \in \mathfrak{g}$.

The following are equivalent:

(i) the curve $g(t)$ satisfies the Euler-Lagrange equations $\mathcal{E} \mathcal{L}(L)(g, \dot{g}, \ddot{g})=0$ on $G$;

(ii) the curve $g(t)$ is a critical point of the action functional $\mathfrak{S}(L)$ for variations $\delta g$ vanishing at the endpoints;

(iii) the curve $v(t)$ solves the Euler-Poincaré equations

$$
\frac{d}{d t} \frac{\partial l}{\partial v}=\operatorname{ad}_{v}^{*} \frac{\partial l}{\partial v}
$$

(iv) the curve $v(t)$ is a critical point of the reduced action functional

$$
\mathfrak{S}_{\text {red }}(l)(v)=\int_{t_{0}}^{t_{1}} l(v(t)) d t
$$

for variations of the form

$$
\delta v=\dot{\eta}+[v, \eta]
$$

where $\eta(t) \in \mathfrak{g}$ is an arbitrary curve that vanishes at the endpoints. These variations $\delta v$ are exactly the variations induced by left translation of arbitrary deformations $g(t, \lambda)$ of the curve $g(t)=g(t, \lambda)$ such that $\delta g\left(t_{i}\right)=0$, for $i=0,1$.

In addition, there is a unique bundle map

$$
\mathcal{E P}(l): 2 \mathfrak{g} \rightarrow \mathfrak{g}^{*}
$$

where $2 \mathfrak{g}:=\mathfrak{g} \oplus \mathfrak{g}$ (in accordance with the definition introduced in the statement of Lemma 3.2.1), such that, for any deformation $v(t, \lambda)=g(t, \lambda)^{-1} \dot{g}(t, \lambda) \in \mathfrak{g}$ induced on $\mathfrak{g}$ by a deformation $g(t, \lambda) \in G$ of $g(t) \in \Omega\left(G ; g_{0}, g_{1}\right)$ keeping the endpoints fixed, and thus $\delta g\left(t_{i}\right)=0$, for $i=0,1$, we have

$$
\mathbf{d S}_{r e d}(l)(v) \cdot \delta v=\int_{t_{0}}^{t_{1}} \mathcal{E} \mathcal{P}(l)(v, \dot{v}) \cdot \eta d t
$$


where, as usual,

$$
\mathbf{d} \mathfrak{S}_{r e d}(l)(v) \cdot \delta v=\left.\frac{d}{d \lambda} \mathfrak{S}_{r e d}(l)(v(t, \lambda))\right|_{\lambda=0}
$$

and $\delta v(t)=\partial v(t, \lambda) /\left.\partial t\right|_{\lambda=0}=\dot{\eta}(t)+[v(t), \eta(t)]$.

The map $\mathcal{E P}(l)$ is called the Euler-Poincaré operator and its expression is given by

$$
\mathcal{E P}(l)(v, \dot{v})=\operatorname{ad}_{v}^{*} \frac{\delta l}{\delta v}-\frac{d}{d t} \frac{\delta l}{\delta v}
$$

where, as before, it is to be understood that the time derivative on the second term is performed formally using the chain rule and that the expression $d v / d t$ is replaced throughout by $\dot{v}$.

The Euler-Poincaré equations can be written simply as $\mathcal{E} \mathcal{P}(l)(v, \dot{v})=0$. The formula $\delta v=\dot{\eta}+[v, \eta]$ represents the most general variation $\delta v$ of $v$ induced by an arbitrary variation $\delta g$ via left translation. The precise relationship is $\eta=g^{-1} \delta g$ and so the condition $\delta g=0$ at the endpoints is equivalent to the condition $\eta=0$ at the endpoints.

\subsection{The Lagrange-Poincaré Operator}

In this section we introduce the Lagrange-Poincaré operator using the same type of technique of reduction of variational principles that was used in the preceding section to define the Euler-Lagrange and the Euler-Poincaré operators.

\section{Reducing the Euler-Lagrange Operator.}

The map $\mathcal{E} \mathcal{L}(L): T^{(2)} Q \rightarrow T^{*} Q$, being $G$ - equivariant, induces a quotient map

$$
[\mathcal{E} \mathcal{L}(L)]_{G}: T^{(2)} Q / G \rightarrow T^{*} Q / G
$$

which depends only on the reduced Lagrangian $l: T Q / G \rightarrow \mathbb{R}$; that is, we can identify $[\mathcal{E} \mathcal{L}(L)]_{G}$ with an operator $\mathcal{E} \mathcal{L}(l)$. This is called the reduced Euler-Lagrange operator and it does not depend on any extra structure on the principal bundle $Q$. However, to write the explicit expressions, which are also physically meaningful, we use the additional structure of a principal connection $A$ on the principal bundle $Q \rightarrow Q / G$ to identify the quotient bundle

$$
T^{(2)} Q / G \quad \text { with } \quad T^{(2)}(Q / G) \times_{Q / G} 2 \tilde{\mathfrak{g}}
$$

and

$$
T^{*} Q / G \quad \text { with } \quad T^{*}(Q / G) \oplus \tilde{\mathfrak{g}}^{*}
$$


using the bundle isomorphisms $\alpha_{A_{2}}$ from Lemma 3.2.2 and $\alpha_{A}$ from Lemma 2.4.2, and also a connection $\nabla$ on $Q / G$ to concretely realize the reduced Euler-Lagrange operator; this will naturally lead us to the Lagrange-Poincaré operator. ${ }^{2}$

\section{Geometry of Reduced Variations.}

A general variation $\delta \bar{v}(t)$ of a given curve $\bar{v}(t)$ in $\tilde{\mathfrak{g}}$ is constructed as follows: choose a family of curves $\bar{v}(t, s)$ in $\tilde{\mathfrak{g}}$ such that $\bar{v}(t, 0)=\bar{v}(t)$ and define

$$
\delta \bar{v}(t)=\left.\frac{\partial \bar{v}(t, s)}{\partial s}\right|_{s=0}
$$

This $\delta \bar{v}(t)$ is, for each $t$, an element of $T \tilde{\mathfrak{g}}$. However, it turns out that we will not need these kinds of general variations $\delta \bar{v}$ subsequently. Instead, we are interested in the special kind of deformations $\bar{v}(t, s)$ of the curve $\bar{v}(t)$ in which the projection $\tilde{\pi}_{G}(\bar{v}(t, s))=x(t, s)$ does not depend on $s$, that is, deformations that take place only in the fiber of $\tilde{\mathfrak{g}}$ over $x(t)=\tilde{\pi}_{G}(\bar{v}(t))$; thus, for each fixed $t$, the curve $s \mapsto \bar{v}(t, s)$ is a curve in the fiber over $x(t)$. Then, since $\tilde{\mathfrak{g}}$ is a vector bundle, the variation $\delta \bar{v}(t)$ induced by such a deformation $\bar{v}(t, s)$, is naturally identified with a curve, also called $\delta \bar{v}(t)$, in $\tilde{\mathfrak{g}}$, a $\tilde{\mathfrak{g}}$-fiber variation, according to the notation introduced in the paragraph Deformation of Curves of $\S 3.1$. We remark that, in the rest of this paper, $\delta \bar{v}$ will always mean a $\tilde{\mathfrak{g}}$-variation, unless explicitly stated otherwise. Also, the meaning of $\delta \bar{v}$ as an element of $T \tilde{\mathfrak{g}}$ will never be used without an explicit previous warning.

Examples of $\tilde{\mathfrak{g}}$-fiber variations $\delta \bar{v}$ are, for instance, the covariant variations $\delta^{A} \bar{v}$ considered in Definition 3.1.3, but, of course, there are more general variations of this type. We have already encountered an easy example of such variations when reviewing the Euler-Poincaré equations. In that case, $Q=G$, the connection $A$ : $T G \rightarrow \mathfrak{g}$ is given by right translation, and $\delta^{A} v(t)=\dot{\eta}(t)+[v(t), \eta(t)]$, for $\eta(t)$ a curve in $\mathfrak{g}$ vanishing at the endpoints.

Of course, in the Euler-Poincaré case, it is obviously true that any deformation of a curve $v(t)$ is a deformation along the fiber, because the base of $\tilde{\mathfrak{g}}$ is a point. However, as we have seen in the Euler-Poincaré Theorem 3.2.4, it is not true that any curve in $\mathfrak{g}$ is induced by a variation $\delta g$ that vanishes at the endpoints; the latter are only the curves of the type $\dot{\eta}(t)+[v(t), \eta(t)]$, for $\eta(t)$ an arbitrary curve in $\mathfrak{g}$ vanishing at the endpoints.

In the study of the Lagrange-Poincaré operator and Lagrange-Poincaré equation we will use variations of curves in $Q / G \oplus \tilde{\mathfrak{g}}$. (As explained in paragraph Bundles of $\S 2.1$, the first summand means the vector bundle over $Q / G$ with zero dimensional fiber). For a given curve $x(t) \oplus \bar{v}(t)$ in $Q / G \oplus \tilde{\mathfrak{g}}$, and a given arbitrary deformation $x(t, \lambda) \oplus \bar{v}(t, \lambda)$, with $x(t, 0) \oplus \bar{v}(t, 0)=x(t) \oplus \bar{v}(t)$, of it, the corresponding covariant

\footnotetext{
${ }^{2}$ In what follows we will assume that the connection $\nabla$ is chosen to be torsion free. For an account of the situation when a connection with torsion is chosen, see Gamboa Saraví and Solomin (2003).
} 
variation $\delta x(t) \oplus \delta^{A} \bar{v}(t)$ is, by definition,

$$
\delta x(t) \oplus \delta^{A} \bar{v}(t)=\left.\left.\frac{\partial x(t, s)}{\partial s}\right|_{s=0} \oplus \frac{D \bar{v}(t, s)}{D s}\right|_{s=0} .
$$

It is clear that $\delta^{A} \bar{v}$ is a $\tilde{\mathfrak{g}}$-fiber variation of $\bar{v}$. The most important example of a covariant variation $\delta x(t) \oplus \delta^{A} \bar{v}(t)$ is the one to be described next. Let $q(t, s)$ be a deformation of a curve $q(t)=q(t, 0)$ in $Q$. This induces a deformation $x(t, s) \oplus \bar{v}(t, s)$ of the curve $x(t) \oplus \bar{v}(t)$ by taking $x(t, s)=[q(t, s)]_{G}$ and $\bar{v}(t, s)=$ $[q(t, s), A(q(t, s), \dot{q}(t, s))]_{G}$, where $\dot{q}(t, s)$ represents the derivative with respect to t. Using Lemma 2.3.4 and Definition 3.1.3, it follows that the covariant variation corresponding to this deformation of $x(t) \oplus \bar{v}(t)$ is $\delta x(t) \oplus \delta^{A} \bar{v}(t)$, where

$$
\delta^{A} \bar{v}(t)=\frac{D[q(t), \eta(t)]_{G}}{D t}+[q(t),[A(q(t), \dot{q}(t)), \eta(t)]]_{G}+\tilde{B}(\delta x(t), \dot{x}(t)),
$$

is an element of $\tilde{\mathfrak{g}}$ for each $t$, with $\eta(t) \in \mathfrak{g}$ an arbitrary curve vanishing at the endpoints. This is a special kind of covariant variation. It is precisely to these kinds of variations that we will apply the usual techniques of the calculus of variations in the next theorems to derive the Lagrange-Poincaré operator and equation. The previous formula may be rewritten as follows, which emphasizes the similarity with the Euler-Poincaré case,

$$
\delta^{A} \bar{v}(t)=\frac{D \bar{\eta}}{D t}(t)+[\bar{v}(t), \bar{\eta}(t)]+\tilde{B}(\delta x(t), \dot{x}(t))
$$

where $\bar{\eta}=[q(t), \eta(t)]_{G}$.

\section{Lagrange-Poincaré Operator.}

We are now ready to state a theorem that introduces the Lagrange-Poincaré operator. Its proof will be contained in the proof of Theorem 3.3.4.

Theorem 3.3.1. Let $L: T Q \rightarrow \mathbb{R}$ be an invariant Lagrangian on the principal bundle $Q$ as before. Choose a principal connection $A$ on $Q$ and identify the bundles $T Q / G$ and $T(Q / G) \oplus \tilde{\mathfrak{g}}$ using the isomorphism $\alpha_{A}$ and also the bundles $T^{(2)} Q / G$ and $T^{(2)}(Q / G) \times_{Q / G} 2 \tilde{\mathfrak{g}}$ using the isomorphism $\alpha_{A_{2}}$, as before. Thus an element $[q, \dot{q}]_{G}$ of $T Q / G$ can be written, equivalently, as an element $(x, \dot{x}, \bar{v})$ of $T(Q / G) \oplus \tilde{\mathfrak{g}}$. Let $l: T(Q / G) \oplus \tilde{\mathfrak{g}} \rightarrow \mathbb{R}$ be the reduced Lagrangian. Then there is a unique bundle map

$$
\mathcal{L} \mathcal{P}(l): T^{(2)}(Q / G) \times_{Q / G} 2 \tilde{\mathfrak{g}} \rightarrow T^{*}(Q / G) \oplus \tilde{\mathfrak{g}}^{*}
$$

such that for any curve $q \in \Omega\left(Q ; q_{0}, q_{1}\right)$ and any variation $\delta q$ of $q$ vanishing at the endpoints, the corresponding reduced curve $[q, \dot{q}]_{G}=(x, \dot{x}, \bar{v})$, where $\bar{v}=$ $[q, A(q, \dot{q})]_{G}$, and covariant variation $\delta x \oplus \delta^{A} \bar{v}$, where

$$
\delta^{A} \bar{v}(t)=\frac{D \bar{\eta}}{D t}(t)+[\bar{v}(t), \bar{\eta}(t)]+\tilde{B}(\delta x(t), \dot{x}(t))
$$


with $\bar{\eta}(t)=[q(t), \eta(t)]_{G}$ and

$$
\delta x(t)=T \pi(\delta q(t))
$$

satisfy

$$
\mathcal{E} \mathcal{L}(L)(q(t), \dot{q}(t), \ddot{q}(t)) \cdot \delta q(t)=\mathcal{L P}(l)(x(t), \dot{x}(t), \bar{v}(t)) \cdot(\delta x(t) \oplus \bar{\eta}(t)) .
$$

Notice that, after all the identifications described at the beginning of the present paragraph, the operator $\mathcal{L} \mathcal{P}(l)$ coincides with the operator $[\mathcal{E L}(L)]_{G}$.

Definition 3.3.2. The 1-form valued bundle map

$$
\mathcal{L P}(l): T^{(2)}(Q / G) \times_{Q / G} 2 \tilde{\mathfrak{g}} \rightarrow T^{*}(Q / G) \oplus \tilde{\mathfrak{g}}^{*}
$$

defined in the preceding theorem will be called the Lagrange-Poincaré operator. The decomposition of the range space for $\mathcal{L P}(l)$ as a direct sum naturally induces a decomposition of the Lagrange-Poincaré operator

$$
\mathcal{L} \mathcal{P}(l)=\operatorname{Hor}(\mathcal{L P})(l) \oplus \operatorname{Ver}(\mathcal{L P})(l)
$$

which define the horizontal Lagrange-Poincaré operator and the vertical Lagrange-Poincaré operator.

The Lagrange-Poincaré equations are, by definition, the equations $\mathcal{L} \mathcal{P}(l)=$ 0. The horizontal Lagrange-Poincaré equation and vertical Lagrange-Poincaré equation are, respectively, the equations

$$
\operatorname{Hor}(\mathcal{L P})(l)=0 \quad \text { and } \quad \operatorname{Ver}(\mathcal{L P})(l)=0 .
$$

In the following paragraph we introduce some additional structure, namely, an arbitrary connection $\nabla$ on the manifold $Q / G$. This will also help us write explicit expressions of $\operatorname{Hor}(\mathcal{L P})(l)$ and $\operatorname{Ver}(\mathcal{L P})(l)$.

The problem of computing the Lagrange-Poincaré equations can be done using any connection, as we remarked earlier and, in addition, the problem can be localized to any local trivialization of the bundle $Q \rightarrow Q / G$. Because of this, one may choose the vector space or trivial connection associated with such a local trivialization of the bundle. Of course we are not assuming that the bundle has a global flat connection.

Explicit formulas for $\operatorname{Hor}(\mathcal{L P})(l)$ and $\operatorname{Ver}(\mathcal{L P})(l)$ in coordinates using any connection can be calculated from what we have developed and are given in $\$ 4.2$. Doing so, one arrives at the coordinate formulas given in Marsden and Scheurle (1993b). We also mention that it is possible to derive these equations from Cendra, Ibort and Marsden (1987) in a straightforward way.

\section{Reduced Covariant Derivatives.}

The question of calculating formulas for $\operatorname{Hor}(\mathcal{L P})(l)$ and $\operatorname{Ver}(\mathcal{L P})(l)$ rests on giving meaning to the partial derivatives

$$
\frac{\partial l}{\partial x}, \quad \frac{\partial l}{\partial \dot{x}} \text { and } \quad \frac{\partial l}{\partial \bar{v}} .
$$


Since $\tilde{\mathfrak{g}}$ and $T(Q / G)$ are vector bundles, we may interpret the last two derivatives in a standard (fiber derivative) way as being elements of the dual bundles $T^{*}(Q / G)$ and $\tilde{\mathfrak{g}}^{*}$, for each choice of $(x, \dot{x}, \bar{v})$ in $T(Q / G) \oplus \tilde{\mathfrak{g}}$. In other words, for given $\left(x_{0}, \dot{x}_{0}, \bar{v}_{0}\right)$ and $\left(x_{0}, x^{\prime}, \bar{v}^{\prime}\right)$ we define

$$
\frac{\partial l}{\partial \dot{x}}\left(x_{0}, \dot{x}_{0}, \bar{v}_{0}\right) \cdot x^{\prime}=\left.\frac{d}{d s}\right|_{s=0} l\left(x_{0}, \dot{x}_{0}+s x^{\prime}, \bar{v}_{0}\right)
$$

and

$$
\frac{\partial l}{\partial \bar{v}}\left(x_{0}, \dot{x}_{0}, \bar{v}_{0}\right) \cdot \bar{v}^{\prime}=\left.\frac{d}{d s}\right|_{s=0} l\left(x_{0}, \dot{x}_{0}, \bar{v}_{0}+s \bar{v}^{\prime}\right) .
$$

To define the derivative $\partial l / \partial x$, one uses the chosen connection $\nabla$ on the manifold $Q / G$, as we will explain next. Let $\left(x_{0}, \dot{x}_{0}, \bar{v}_{0}\right)$ be a given element of $T(Q / G) \oplus \tilde{\mathfrak{g}}$. For any given curve $x(s)$ on $Q / G$, let $(x(s), \bar{v}(s))$ be the horizontal lift of $x(s)$ with respect to the connection $\tilde{\nabla}^{A}$ on $\tilde{\mathfrak{g}}$ (see $\left.(2.3 .1)\right)$ such that $(x(0), \bar{v}(0))=\left(x_{0}, \bar{v}_{0}\right)$ and let $(x(s), u(s))$ be the horizontal lift of $x(s)$ with respect to the connection $\nabla$ such that $(x(0), u(0))=\left(x_{0}, \dot{x}_{0}\right)$. (Notice that, in general, $(x(s), u(s))$ is not the tangent vector $(x(s), \dot{x}(s))$ to $x(s)$.)

Thus, $(x(s), u(s), \bar{v}(s))$ is a horizontal curve with respect to the connection $C=$ $\nabla \oplus \tilde{\nabla}^{A}$ naturally defined on $T(Q / G) \oplus \tilde{\mathfrak{g}}$ in terms of the connection $\nabla$ on $T(Q / G)$ and the connection $\tilde{\nabla}^{A}$ on $\tilde{\mathfrak{g}}$.

Definition 3.3.3. The covariant derivative of $l$ with respect to $x$ at $\left(x_{0}, \dot{x}_{0}, \bar{v}_{0}\right)$ in the direction of $(x(0), \dot{x}(0))$ is defined by

$$
\frac{\partial^{C} l}{\partial x}\left(x_{0}, \dot{x}_{0}, \bar{v}_{0}\right)(x(0), \dot{x}(0))=\left.\frac{d}{d s}\right|_{s=0} l(x(s), u(s), \bar{v}(s)) .
$$

We shall often write

$$
\frac{\partial^{C} l}{\partial x} \equiv \frac{\partial l}{\partial x}
$$

whenever there is no danger of confusion.

The covariant derivative on a given vector bundle, for instance $\tilde{\mathfrak{g}}$, induces a corresponding covariant derivative on the dual bundle, in our case $\tilde{\mathfrak{g}}^{*}$. More precisely, let $\alpha(t)$ be a curve in $\tilde{\mathfrak{g}}^{*}$. We define the covariant derivative of $\alpha(t)$ in such a way that for any curve $\bar{v}(t)$ on $\tilde{\mathfrak{g}}$, such that both $\alpha(t)$ and $\bar{v}(t)$ project on the same curve $x(t)$ on $Q / G$, we have

$$
\frac{d}{d t}\langle\alpha(t), \bar{v}(t)\rangle=\left\langle\frac{D \alpha(t)}{D t}, \bar{v}(t)\right\rangle+\left\langle\alpha(t), \frac{D \bar{v}(t)}{D t}\right\rangle .
$$

Likewise we can define the covariant derivative in the vector bundle $T^{*}(Q / G)$. Then we obtain a covariant derivative on the vector bundle $T^{*}(Q / G) \oplus \tilde{\mathfrak{g}}^{*}$.

It is in the sense of this definition that terms like

$$
\frac{D}{D t} \frac{\partial l}{\partial \dot{x}}
$$


in the second equation (which defines the horizontal Lagrange-Poincaré operator) and

$$
\frac{D}{D t} \frac{\partial l}{\partial \bar{v}}
$$

in the first equation (which defines the vertical Lagrange-Poincaré equation) of the following theorem should be interpreted. In this case $D / D t$ means the covariant derivative in the bundle $T^{*}(Q / G)$. In the first equation $D / D t$ is the covariant derivative in the bundle $\tilde{\mathfrak{g}}^{*}$.

\section{Reduced Variational Principles \& the Lagrange-Poincaré Equations.}

The main result in this section is the following theorem. Its proof also contains the proof of the preceding theorem.

Theorem 3.3.4. Assume the hypothesis of Theorem 3.3.1 Then:

the vertical Lagrange-Poincaré operator is given by

$$
\operatorname{Ver}(\mathcal{L P})(l) \cdot \bar{\eta}=\left(-\frac{D}{D t} \frac{\partial l}{\partial \bar{v}}(x, \dot{x}, \bar{v})+\operatorname{ad}_{\bar{v}}^{*} \frac{\partial l}{\partial \bar{v}}(x, \dot{x}, \bar{v})\right) \cdot \bar{\eta}
$$

or simply,

$$
\operatorname{Ver}(\mathcal{L P})(l)=\left(-\frac{D}{D t} \frac{\partial l}{\partial \bar{v}}(x, \dot{x}, \bar{v})+\operatorname{ad}_{\bar{v}}^{*} \frac{\partial l}{\partial \bar{v}}(x, \dot{x}, \bar{v})\right)
$$

and the horizontal Lagrange-Poincaré operator is given by

$$
\operatorname{Hor}(\mathcal{L P})(l) \cdot \delta x=\left(\frac{\partial l}{\partial x}(x, \dot{x}, \bar{v})-\frac{D}{D t} \frac{\partial l}{\partial \dot{x}}(x, \dot{x}, \bar{v})\right) \delta x-\frac{\partial l}{\partial \bar{v}}(x, \dot{x}, \bar{v}) \tilde{B}(x)(\dot{x}, \delta x),
$$

or simply,

$$
\operatorname{Hor}(\mathcal{L P})(l)=\left(\frac{\partial l}{\partial x}(x, \dot{x}, \bar{v})-\frac{D}{D t} \frac{\partial l}{\partial \dot{x}}(x, \dot{x}, \bar{v})\right)-\frac{\partial l}{\partial \bar{v}}(x, \dot{x}, \bar{v}) \tilde{B}(x)(\dot{x}, .) .
$$

Proof. To compute the vertical and horizontal Lagrange-Poincaré operator, it suffices to consider variations $\delta^{A} \bar{v}$ of a curve $x(t) \oplus \bar{v}(t)$ corresponding to vertical and horizontal variations $\delta q$ of a curve $q \in \Omega\left(Q, q_{0}, q_{1}\right)$. The computations below will show that these variations suffice to give us the variational principle in the directions of the two summands in $\delta x \oplus \bar{\eta} \in T(Q / G) \oplus \tilde{\mathfrak{g}}$.

First, consider variations $\delta^{A} \bar{v}$ of a curve $x(t) \oplus \bar{v}(t)$ corresponding to vertical variations $\delta q$ of a curve $q$. We have

$$
0=\delta \int_{t_{0}}^{t_{1}} l(x, \dot{x}, \bar{v}) d t=\int_{t_{0}}^{t_{1}} \frac{\partial l}{\partial \bar{v}}(x, \dot{x}, \bar{v}) \delta^{A} \bar{v} d t
$$


According to Lemma 3.1.4 with $\bar{v}=[q, v]_{G}$ we obtain, for all curves $\eta(t) \in \mathfrak{g}$ such that $\eta\left(t_{i}\right)=0$ for $i=1,2$, the equation

$$
\begin{aligned}
0 & =\int_{t_{0}}^{t_{1}}\left\langle\frac{\partial l}{\partial \bar{v}}, \frac{D[q, \eta]_{G}}{D t}+[q,[v, \eta]]_{G}\right\rangle d t \\
& =\int_{t_{0}}^{t_{1}}\left\langle-\frac{D}{D t} \frac{\partial l}{\partial \bar{v}}+\operatorname{ad}_{\bar{v}}^{*} \frac{\partial l}{\partial \bar{v}},[q, \eta]_{G}\right\rangle d t
\end{aligned}
$$

Arbitrariness of $\eta$ then yields arbitrariness of $\bar{\eta}=[q, \eta]_{G}$, so we get

$$
\operatorname{Ver}(\mathcal{L P})(l)=-\frac{D}{D t} \frac{\partial l}{\partial \bar{v}}(x, \dot{x}, \bar{v})+\operatorname{ad}_{\bar{v}}^{*} \frac{\partial l}{\partial \bar{v}}(x, \dot{x}, \bar{v}) .
$$

Now consider variations $\delta x \oplus \delta^{A} \bar{v}$ corresponding to horizontal variations $\delta q$. Then we have, for all $\delta x$ with $\delta x\left(t_{i}\right)=0$, for $i=0,1$

$$
\delta \int_{t_{0}}^{t_{1}} l(x, \dot{x}, \bar{v}) d t=\int_{t_{0}}^{t_{1}}\left(\frac{\partial l}{\partial x} \delta x+\frac{\partial l}{\partial \dot{x}} \delta \dot{x}+\frac{\partial l}{\partial \bar{v}} \delta^{A} \bar{v}\right) d t .
$$

Integration by parts and Lemma 3.1.7 with $\bar{v}=[q, v]_{G}$ gives

$$
\delta \int_{t_{0}}^{t_{1}} l(x, \dot{x}, \bar{v}) d t=\int_{t_{0}}^{t_{1}}\left[\left(\frac{\partial l}{\partial x}-\frac{D}{D t} \frac{\partial l}{\partial \dot{x}}\right)(x, \dot{x}, \bar{v}) \delta x-\frac{\partial l}{\partial \bar{v}}(x, \dot{x}, \bar{v}) \tilde{B}(x)(\dot{x}, \delta x)\right] d t .
$$

Integration by parts of the term $(\partial l / \partial \dot{x}) \delta \dot{x}$ is justified by showing that

$$
\delta \dot{x}=\frac{D}{D \lambda} \frac{\partial x}{\partial t}=\frac{D}{D t} \frac{\partial x}{\partial \lambda},
$$

which can be done, for example, by using Gaussian coordinates relative to the connection $\nabla$ at each point $x(t)$. Arbitrariness of $\delta x$ then yields

$$
\operatorname{Hor}(\mathcal{L P})(l)(x, \dot{x}, \bar{v})=\frac{\partial l}{\partial x}(x, \dot{x}, \bar{v})-\frac{D}{D t} \frac{\partial l}{\partial \dot{x}}(x, \dot{x}, \bar{v})-\frac{\partial l}{\partial \bar{v}}(x, \dot{x}, \bar{v}) \mathbf{i}_{\dot{x}} \tilde{B}(x) .
$$

\subsection{The Reduced Variational Principle}

Now we turn to the reduction of the variational principle. As we stated at the beginning of this section, Hamilton's principle for a $G$-invariant $L$ is equivalent to a reduced variational principle for $l$ with respect to a reduced set of curves $\left[\Omega\left(Q ; q_{0}, q_{1}\right)\right]_{G}$. Translated to the concrete realizations of our reduced bundles using the map $\alpha_{A}$, this reads as follows.

The following conditions are equivalent: 
(i) Hamilton's principle holds: the curve $q(t)$ is a critical point of the action functional

$$
\int_{t_{0}}^{t_{1}} L(q, \dot{q}) d t
$$

on $\Omega\left(Q ; q_{0}, q_{1}\right)$;

(ii) the reduced variational principle holds: the curve $(x(t), \bar{v}(t))$ is a critical point of the action functional

$$
\int_{t_{0}}^{t_{1}} l(x(t), \dot{x}(t), \bar{v}(t)) d t
$$

on the reduced family of curves $\Omega\left(\alpha_{A}\left(\left[\Omega\left(Q ; q_{0}, q_{1}\right)\right]_{G}\right)\right)$.

Now comes a main theorem which summarizes what we have done so far.

Theorem 3.4.1. The following conditions are equivalent:

(i) Hamilton's principle holds: the curve $q(t)$ is a critical point of the action functional

$$
\int_{t_{0}}^{t_{1}} L(q, \dot{q}) d t
$$

on $\Omega\left(Q ; q_{0}, q_{1}\right)$, that is,

$$
\delta \int_{t_{0}}^{t_{1}} L(q, \dot{q}) d t=0
$$

for arbitrary variations $\delta q$ of the curve $q$ such that $\delta q\left(t_{i}\right)=0$, for $i=0,1$.

(ii) The reduced variational principle holds: the curve $x(t) \oplus \bar{v}(t)$ is a critical point of the action functional

$$
\int_{t_{0}}^{t_{1}} l(x(t), \dot{x}(t), \bar{v}(t)) d t
$$

on the reduced family of curves $\alpha_{A}\left(\left[\Omega\left(Q ; q_{0}, q_{1}\right)\right]_{G}\right)$, that is,

$$
\delta \int_{t_{0}}^{t_{1}} l(x(t), \dot{x}(t), \bar{v}(t)) d t=0,
$$

for variations $\delta x \oplus \delta^{A} \bar{v}$ of the curve $x(t) \oplus \bar{v}(t)$, where $\delta^{A} \bar{v}$ has the form

$$
\delta^{A} \bar{v}=\frac{D \bar{\eta}}{D t}+[\bar{v}, \bar{\eta}]+\tilde{B}(\delta x, \dot{x}),
$$

with the boundary conditions $\delta x\left(t_{i}\right)=0$ and $\bar{\eta}\left(t_{i}\right)=0$, for $i=0,1$. If $\bar{v}=$ $[q, v]_{G}$ with $v=A(q, \dot{q})$ then $\bar{\eta}$ can be always written $\bar{\eta}=[q, \eta]_{G}$, and the condition $\bar{\eta}\left(t_{i}\right)=0$ for $i=0,1$ is equivalent to the condition $\eta\left(t_{i}\right)=0$ for $i=0,1$. 
Also, if $x(t)=[q]_{G}$ and $\bar{v}=[q, v]_{G}$ where $v=A(q, \dot{q})$, then variations $\delta x \oplus \delta^{A} \bar{v}$ such that

$$
\begin{aligned}
\delta^{A} \bar{v} & =\frac{D \bar{\eta}}{D t}+[\bar{v}, \bar{\eta}] \\
& \equiv \frac{D[q, \eta]_{G}}{D t}+[q,[v, \eta]]_{G}
\end{aligned}
$$

with $\bar{\eta}\left(t_{i}\right)=0$ (or, equivalently, $\eta\left(t_{i}\right)=0$ ) for $i=0,1$ correspond exactly to vertical variations $\delta q$ of the curve $q$ such that $\delta q\left(t_{i}\right)=0$ for $i=0,1$, while variations $\delta x \oplus \delta^{A} \bar{v}$ such that

$$
\delta^{A} \bar{v}=\tilde{B}(\delta x, \dot{x})
$$

with $\delta x\left(t_{i}\right)=0$ for $1=0,1$, correspond exactly to horizontal variations $\delta q$ of the curve $q$ such that $\delta q\left(t_{i}\right)=0$.

(iii) The following vertical Lagrange-Poincaré equations, corresponding to vertical variations, hold:

$$
\frac{D}{D t} \frac{\partial l}{\partial \bar{v}}(x, \dot{x}, \bar{v})=\operatorname{ad}_{\bar{v}}^{*} \frac{\partial l}{\partial \bar{v}}(x, \dot{x}, \bar{v})
$$

and the horizontal Lagrange-Poincaré equations, corresponding to horizontal variations, hold:

$$
\frac{\partial l}{\partial x}(x, \dot{x}, \bar{v})-\frac{D}{D t} \frac{\partial l}{\partial \dot{x}}(x, \dot{x}, \bar{v})=\left\langle\frac{\partial l}{\partial \bar{v}}(x, \dot{x}, \bar{v}), \mathbf{i}_{\dot{x}} \tilde{B}(x)\right\rangle .
$$

\section{Remarks.}

1. The operators $\mathcal{E} \mathcal{L}(l), \operatorname{Hor}(\mathcal{L P})(l)$ and $\operatorname{Ver}(\mathcal{L P})(l)$ depend on the (principal) connection $A$ on the principal bundle $Q$ but not on the connection $\nabla$ on $Q / G$. It is only the explicit expressions of $\operatorname{Hor}(\mathcal{L P})(l)$ and $\operatorname{Ver}(\mathcal{L P})(l)$ that appear in Theorem 3.3.4 that depend on $\nabla$. As we have remarked previously, in local coordinates it is often convenient to choose $\nabla$ to be simply the usual Euclidean, or vector space connection.

2. Important particular cases of Theorems 3.3.1 and 3.3.4 occur when $G=$ $Q$ and also when $G=\{e\}$. If $G=Q$ then the operator $\operatorname{Hor}(\mathcal{L P})(l)$ is 0 and $\operatorname{Ver}(\mathcal{L P})(l)$ is the Euler-Poincaré operator, as we saw before. Thus, in a sense, the vertical Lagrange-Poincaré operator in the bundle $\tilde{\mathfrak{g}}$ is a covariant version of the usual Euler-Poincaré operator on a Lie algebra. If $G=\{e\}$ then $\operatorname{Ver}(\mathcal{L P})(l)$ is $0, L=l$ and $\operatorname{Hor}(\mathcal{L P})(l)=\mathcal{E} \mathcal{L}(L)$ is the usual Euler-Lagrange operator. 


\section{4 \\ Wong's Equations and Coordinate Formulas}

To illustrate the Lagrange-Poincaré theory that we have developed, we first consider an interesting example, that of Wong's equations. Secondly, in this section we give coordinate expressions for the Lagrange-Poincaré equations. Wong's equations are first done intrinsically and then are used to illustrate the coordinate formulas.

\subsection{Wong's Equations}

\section{Context of Wong's Equations.}

Wong's equations arise in at least two different interesting contexts. The first of these, in the work of Wong (1970), Sternberg (1977), Weinstein (1978) and Montgomery (1984), concerns the dynamics of a colored particle in a Yang-Mills field. The second context is that of the falling cat theorem of Montgomery [1990, 1993]. For a direct proof of the falling cat theorem using the ideas of Lagrangian reduction, see Koon and Marsden (1997a) and Cendra, Holm, Marsden and Ratiu (1998).

\section{Abstract Setting.}

Let $(X, g)$ be a given Riemannian manifold and let $\nabla$ be the corresponding LeviCivita connection. Let $G$ be a compact Lie group with a bi-invariant Riemannian metric $\kappa$. Let $\pi: Q \rightarrow X$ be a principal bundle with structure group $G$ acting on the left, let $A$ be a principal connection on $Q$, and let $B$ be the curvature of $A$. 
Now define the Lagrangian $L: T Q \rightarrow \mathbb{R}$ by

$$
L(q, \dot{q})=\frac{1}{2} \kappa(A(q, \dot{q}), A(q, \dot{q}))+\frac{1}{2} g(\pi(q))(T \pi(q, \dot{q}), T \pi(q, \dot{q})) .
$$

This Lagrangian is $G$-invariant and our object is to carry out the constructions for Lagrangian reduction as described in the preceding sections to this situation.

We note that in the special case of $G=S^{1}$, this Lagrangian is the KaluzaKlein Lagrangian for the motion of a particle in a magnetic field. In this case, the constructions are done directly in Marsden and Ratiu (1999). More generally, this Lagrangian is the Kaluza-Klein Lagrangian for particles in a Yang-Mills field $A$.

\section{Construction of the Reduced Bundle.}

An element of $\tilde{\mathfrak{g}}$ has the form $\bar{v}=[q, v]_{G}$ where $q \in Q$ and $v \in \mathfrak{g}$. Since $\kappa$ is biinvariant, its restriction to $\mathfrak{g}$ is Ad-invariant, and so we can define the fiber metric $k$ on $\tilde{\mathfrak{g}}$ by

$$
k\left([q, u]_{G},[q, v]_{G}\right)=\kappa(u, v) .
$$

The reduced bundle is $T(Q / G) \oplus \tilde{\mathfrak{g}} \equiv T X \oplus \tilde{\mathfrak{g}}$ and a typical element of it is denoted $(x, \dot{x}, \bar{v})$. The reduced Lagrangian is given by

$$
l(x, \dot{x}, \bar{v})=\frac{1}{2} k(\bar{v}, \bar{v})+\frac{1}{2} g(x)(x, \dot{x}) .
$$

\section{Calculation of the Reduced Equations.}

Now we will write the vertical and horizontal Lagrange-Poincaré equations. We start by writing the vertical Lagrange-Poincaré equation from Theorem 3.3.1 as follows:

$$
\left(-\frac{D}{D t} \frac{\partial l}{\partial \bar{v}}(x, \dot{x}, \bar{v})+\operatorname{ad}_{\bar{v}}^{*} \frac{\partial l}{\partial \bar{v}}(x, \dot{x}, \bar{v})\right) \cdot \bar{\eta}=0
$$

for all $\bar{\eta} \in \tilde{\mathfrak{g}}$. We first note that

$$
\frac{\partial l}{\partial \bar{v}}(x, \dot{x}, \bar{v})=k(\bar{v}, \cdot)
$$

and hence

$$
\left(\operatorname{ad}_{\bar{v}}^{*} \frac{\partial l}{\partial \bar{v}}(x, \dot{x}, \bar{v})\right) \bar{\eta}=k(\bar{v},[\bar{v}, \bar{\eta}])=0,
$$

since $\kappa$ and hence $k$ are bi-invariant. Thus, the vertical Lagrange-Poincaré equation is

$$
\frac{D}{D t} k(\bar{v}, \cdot)=0
$$

which is one of Wong's equations, namely the charge equation. We will see this explicitly in coordinates later. 
From Theorem 3.4.1, the horizontal Lagrange-Poincaré equation is

$$
\frac{\partial l}{\partial x}(x, \dot{x}, \bar{v})-\frac{D}{D t} \frac{\partial l}{\partial \dot{x}}(x, \dot{x}, \bar{v})=\left\langle\frac{\partial l}{\partial \bar{v}}(x, \dot{x}, \bar{v}), \mathbf{i}_{\dot{x}} \tilde{B}(x)\right\rangle .
$$

Perhaps the easiest way to work out this expression is to do so in a local trivialization of the principal bundle, which induces a corresponding trivialization of $\tilde{\mathfrak{g}}$. In such a local trivialization, the metric $k$ is independent of the base point $x$. Making use of the vertical equation, the left hand side of the preceding equation becomes the usual Euler-Lagrange expression. Note that this expression is independent of which affine connection is used on $X$. It is well known that the Euler-Lagrange expression for the kinetic energy on $X$ gives the covariant acceleration $\nabla_{\dot{x}} \dot{x}$ using the LeviCivita connection for the metric on $X$. Therefore, the horizontal Lagrange-Poincaré equation becomes

$$
\left(\nabla_{\dot{x}} \dot{x}\right)^{b}=-k(\bar{v}, \tilde{B}(x)(\dot{x}, \cdot)),
$$

which is the second Wong equation.

\subsection{The Local Vertical and Horizontal Equations}

In this section we shall derive local formulas (that is, for a local trivialization of the principal bundle) of both the vertical and the horizontal Lagrange-Poincaré operator. The expressions that we obtain coincide with or can be easily derived from the ones obtained in Cendra and Marsden (1987), Cendra, Ibort and Marsden (1987) and Marsden and Scheurle (1993b), with some changes in the notation. We start with the covariant formulas for the vertical and horizontal Lagrange-Poincaré operators described in the previous theorems and the local expressions are then easily derived.

\section{Local Vertical Lagrange-Poincaré Equation.}

We now derive local coordinate expressions for the vertical Lagrange-Poincaré equations.

Suppose that $Q$ has dimension $n$, so that $Q / G$ has dimension $r=n-\operatorname{dim} G$. We choose a local trivialization of the principal bundle $Q \rightarrow Q / G$ to be $X \times G$, where $X$ is an open set in $\mathbb{R}^{r}$. Thus, we consider the trivial principal bundle $\pi: X \times G \rightarrow X$ with structure group $G$ acting only on the second factor by left multiplication. Let $e$ be the neutral element of $G$ and let $A$ be a given principal connection on the bundle $Q \rightarrow Q / G$, or, in local representation, on the bundle $X \times G \rightarrow X$. We shall also assume that there are local coordinates $x^{\alpha}, \alpha=1, \ldots, r$, in $X$ and that we choose the standard flat connection on $X$. Then, at any tangent vector $(x, g, \dot{x}, \dot{g}) \in T_{(x, g)}(X \times G)$ we have

$$
A(x, g, \dot{x}, \dot{g})=\operatorname{Ad}_{g}\left(A_{e}(x) \cdot \dot{x}+v\right)
$$


where $A_{e}$ is the $\mathfrak{g}$-valued 1 -form on $X$ defined by $A_{e}(x) \cdot \dot{x}=A(x, e, \dot{x}, 0)$ and $v=g^{-1} \dot{g}$. The vector bundle isomorphism $\alpha_{A}$ in this case becomes

$$
\alpha_{A}\left([x, g, \dot{x}, \dot{g}]_{G}\right)=(x, \dot{x}) \oplus \bar{v}
$$

where $\bar{v}=\left(x, A_{e}(x) \cdot \dot{x}+v\right)$. We will often write $(x, \dot{x}, \bar{v})$ instead of $(x, \dot{x}) \oplus \bar{v}$, and sometimes, simply $\bar{v}=A_{e}(x) \cdot \dot{x}+v$. Let us choose maps

$$
\mathbf{e}_{b}: X \rightarrow \mathfrak{g}
$$

where $b=1, \ldots, \operatorname{dim}(G)$, such that, for each $x \in X$, is a basis of $\mathfrak{g}$. For each $b=$ $1, . ., \operatorname{dim}(G)$, let $\overline{\mathbf{e}}_{b}(x)$ be the section of $\tilde{\mathfrak{g}}$ given by $\overline{\mathbf{e}}_{b}(x)=\left[x, e, \mathbf{e}_{b}(x)\right]_{G} \equiv\left(x, \mathbf{e}_{b}(x)\right)$. Let us call $p=p(x, \dot{x}, \bar{v})$ the vertical body momentum of the reduced system, that is, by definition,

$$
p(x, \dot{x}, \bar{v})=\frac{\partial l}{\partial \bar{v}}(x, \dot{x}, \bar{v}) .
$$

We denote the components of $p$ by $p_{b}=p\left(\overline{\mathbf{e}}_{b}\right) \equiv\left\langle p, \overline{\mathbf{e}}_{b}\right\rangle$. We want to find an equation for the evolution of $p_{b}$. We have

$$
\begin{aligned}
\frac{d}{d t} p_{b} & =\frac{d}{d t}\left\langle p, \overline{\mathbf{e}}_{b}\right\rangle \\
& =\left\langle\frac{D}{D t} p, \overline{\mathbf{e}}_{b}\right\rangle+\left\langle p, \frac{D}{D t} \overline{\mathbf{e}}_{b}\right\rangle .
\end{aligned}
$$

Using the vertical Lagrange-Poincaré equation we obtain, immediately,

$$
\begin{aligned}
\left\langle\frac{D}{D t} p, \overline{\mathbf{e}}_{b}\right\rangle & =\left\langle p,\left[\bar{v}, \overline{\mathbf{e}}_{b}\right]\right\rangle \\
& =\left\langle p,\left[A_{e}(x) \cdot \dot{x}+v, \mathbf{e}_{b}\right]\right\rangle .
\end{aligned}
$$

Lemma 2.3.4 gives the general formula for calculating the covariant derivative of a given curve $[q(t), \xi(t)]_{G}$ in $\tilde{\mathfrak{g}}$,

$$
\frac{D[q(t), \xi(t)]_{G}}{D t}=[q(t),-[A(q(t), \dot{q}(t)), \xi(t)]+\dot{\xi}(t)]_{G} .
$$

We are going to apply this formula for the case of the curve

$$
\overline{\mathbf{e}}_{b}(x(t))=\left[x(t), e, \mathbf{e}_{b}(x(t))\right]_{G} \equiv\left(x(t), \mathbf{e}_{b}(x(t))\right)
$$

in $\tilde{\mathfrak{g}}$. Note that the tangent vector to the curve $q(t) \equiv(x(t), e)$ is $(q(t), \dot{q}(t)) \equiv$ $(x(t), e, \dot{x}(t), 0)$, and therefore $A(q(t), \dot{q}(t)) \equiv A_{e}(x(t)) \cdot \dot{x}$. Using equation (4.2.4) we obtain

$$
\begin{aligned}
\frac{D}{D t} \overline{\mathbf{e}}_{b} & =\left[x, e,-\left[A_{e}(x) \cdot \dot{x}, \mathbf{e}_{b}\right]+\dot{\mathbf{e}}_{b}\right]_{G} \\
& \equiv\left(x,-\left[A_{e}(x) \cdot \dot{x}, \mathbf{e}_{b}\right]+\dot{\mathbf{e}}_{b}\right) .
\end{aligned}
$$


Using equations (4.2.2), (4.2.3) and (4.2.5) we obtain the equation

$$
\frac{d p_{b}}{d t}=\left\langle p,\left[v, \mathbf{e}_{b}\right]+\dot{\mathbf{e}}_{b}\right\rangle .
$$

Using this equation we can easily find an expression in coordinates for the vertical Lagrange-Poincaré equation. Let us choose the functions $\mathbf{e}_{b}(x)$ to be constant functions; therefore, we have $\dot{\mathbf{e}}_{b}=0$ and the equation for the evolution of $p_{b}$ becomes

$$
\frac{d p_{b}}{d t}=\left\langle p,\left[v, \mathbf{e}_{b}\right]\right\rangle
$$

Recall that $\bar{v}-A_{e}(x) \cdot \dot{x}=v$, and thus the equation (4.2.7) becomes

$$
\frac{d p_{b}}{d t}=\left\langle p,\left[\bar{v}-A_{e}(x) \cdot \dot{x}, \mathbf{e}_{b}\right]\right\rangle .
$$

Let $C_{b d}^{a}$ be the structure constants of the Lie algebra $\mathfrak{g}$. For the given local coordinates $x^{\alpha}$ in $X$ let $A_{\alpha}^{a}(x)$ be the coefficients of $A_{e}$, that is, by definition, $\left(A_{e}(x)\right.$. $\dot{x})^{a} \mathbf{e}_{a}=A_{\alpha}^{a}(x) \dot{x}^{\alpha} \mathbf{e}_{a}$. Then equation (4.2.8) becomes the "Poincaré part" of the Lagrange-Poincaré equations:

$$
\frac{d p_{b}}{d t}=p_{a}\left(C_{d b}^{a} \bar{v}^{d}-C_{d b}^{a} A_{\alpha}^{d} \dot{x}^{\alpha}\right)
$$

This equation coincides with equation (5.3.3) of Bloch, Krishnaprasad, Marsden and Murray (1996) and equation (3.2) in Koon and Marsden (1997c). It also agrees, up to some sign problems, with that in Marsden and Scheurle (1993b) and is also implicit in Cendra, Ibort and Marsden (1987).

Equation (4.2.9) reduces to the Euler-Poincaré equation in the case that the base is a point. If we think of the variables evolving as $(x, \dot{x}, \bar{v})$, and, thinking of $p_{b}$ as a function of these variables, using the definition of $p_{b}$, we can write this equation as

$$
\frac{d p_{b}(x, \dot{x}, \bar{v})}{d t}=p_{a}(x, \dot{x}, \bar{v})\left(C_{d b}^{a} \bar{v}^{d}-C_{d b}^{a} A_{\alpha}^{d}(x) \dot{x}^{\alpha}\right) .
$$

Notice that using the variable $v$, which is obtained by $\bar{v}=A_{e}(x) \cdot \dot{x}+v$, we can write the equation as

$$
\frac{d p_{b}\left(x, \dot{x}, A_{e}(x) \cdot \dot{x}+v\right)}{d t}=p_{a}\left(x, \dot{x}, A_{e}(x) \dot{x}+v\right) C_{d b}^{a} v^{d} .
$$

Observe that one can calculate $p_{b}$ in equation (4.2.1) by taking the derivative of $l$ with respect to either $\bar{v}^{b}$ or $v^{b}$.

In Bloch, Krishnaprasad, Marsden and Murray (1996), the variable $\bar{v}^{b}$ is called $\Omega^{b}$ and is interpreted as the locked body angular velocity. This variable is intrinsic, given the choice of a connection, whereas $v^{b}$ depends on the local trivialization. In fact, the form of equation (4.2.11) is dependent on choosing a local trivialization. 


\section{Local Horizontal Lagrange-Poincaré Equation.}

To calculate the local horizontal Lagrange-Poincaré equation we shall first calculate

$$
\frac{\partial^{C} l}{\partial x}(x, \dot{x}, \bar{v}) \cdot \delta x
$$

By Definition 3.3.3 of the notation $\partial^{C} l / \partial x$, we have

$$
\frac{\partial^{C} l}{\partial x}(x, \dot{x}, \bar{v}) \cdot \delta x=\left.\frac{d}{d \lambda} l(x+\lambda \delta x, \dot{x}, \bar{w}(\lambda))\right|_{\lambda=0},
$$

where $\bar{w}(\lambda)$ is a curve such that $\bar{w}(\lambda) \in \tilde{\mathfrak{g}}_{x+\lambda \delta x}$ for each $\lambda, \bar{w}(0)=\bar{v}$ and

$$
\frac{D \bar{w}(\lambda)}{D \lambda}=0 \text {. }
$$

If $\bar{w}(\lambda)=(x+\lambda \delta x, e, w(\lambda))$, we can deduce from equation (4.2.4)

$$
\frac{D \bar{w}(\lambda)}{D \lambda}=\left(x(\lambda), e,-[A(x(\lambda)) \cdot \delta x, w(\lambda)]+\frac{d w(\lambda)}{d \lambda}\right) .
$$

Therefore we must have

$$
\frac{d w(\lambda)}{d \lambda}=[A(x(\lambda)) \cdot \delta x, w(\lambda)]
$$

We then obtain

$$
\frac{\partial^{C} l}{\partial x}(x, \dot{x}, \bar{v}) \cdot \delta x=\frac{\partial l}{\partial x}(x, \dot{x}, \bar{v}) \cdot \delta x+\frac{\partial l}{\partial \bar{v}}(x, \dot{x}, \bar{v}) \cdot[A(x(\lambda)) \cdot \delta x, \bar{w}(\lambda)] .
$$

On the other hand, it is easy to see that $\tilde{B}(x)(\dot{x}, \delta x)=(x, e, B(x, e)(\dot{x}, \delta x))$. Then the horizontal Lagrange-Poincaré operator is

$$
\left(\frac{\partial l}{\partial x}(x, \dot{x}, \bar{v})-\frac{d}{d t} \frac{\partial l}{\partial \dot{x}}(x, \dot{x}, \bar{v})\right) \delta x=\frac{\partial l}{\partial \bar{v}}(x, \dot{x}, \bar{v})(B(x, e)(\dot{x}, \delta x)+[\bar{v}, A(x) \cdot \delta x]) .
$$

As we did with the vertical Lagrange-Poincaré operator, it is convenient to rewrite this equation explicitly in coordinates and we easily obtain

$$
\frac{\partial l}{\partial x^{\alpha}}(x, \dot{x}, \bar{v})-\frac{d}{d t} \frac{\partial l}{\partial \dot{x}^{\alpha}}(x, \dot{x}, \bar{v})=\frac{\partial l}{\partial \bar{v}^{a}}(x, \dot{x}, \bar{v})\left(B_{\beta \alpha}^{a}(x, e) \dot{x}^{\beta}+C_{d b}^{a} \bar{v}^{d} A_{\alpha}^{b}(x)\right),
$$

where a fixed basis $\overline{\mathbf{e}}_{a}$ of $\tilde{\mathfrak{g}}$ has been chosen and, in this basis, $\bar{v}=\bar{v}^{a} \overline{\mathbf{e}}_{a}$. This equation coincides with equation (5.3.2) of Bloch, Krishnaprasad, Marsden and Murray (1996) and equation (3.1) of Koon and Marsden (1997c). We remark that in these papers the convention for the sign of the curvature $B_{\alpha \beta}^{a}$ is the opposite to the one used in this paper. 


\section{Summary.}

The Lagrange-Poincaré equations in coordinates have been shown to be (dropping the independent variables from the notation)

$$
\begin{gathered}
\frac{d p_{b}}{d t}=p_{a}\left(C_{d b}^{a} \bar{v}^{d}-C_{d b}^{a} A_{\alpha}^{d} \dot{x}^{\alpha}\right) \\
\frac{\partial l}{\partial x^{\alpha}}-\frac{d}{d t} \frac{\partial l}{\partial \dot{x}^{\alpha}}=\frac{\partial l}{\partial \bar{v}^{a}}\left(B_{\beta \alpha}^{a} \dot{x}^{\beta}+C_{d b}^{a} \bar{v}^{d} A_{\alpha}^{b}\right),
\end{gathered}
$$

where, as usual, a summation is implied over repeated indices.

In the special case when the bundle $Q \rightarrow Q / G$ is endowed with a trivial connection in local representation, i.e., $A=0$, these equations simply become

$$
\begin{aligned}
& \frac{d p_{b}}{d t}-p_{a} C_{d b}^{a} \bar{v}^{d}=0 \\
& \frac{\partial l}{\partial x^{\alpha}}-\frac{d}{d t} \frac{\partial l}{\partial \dot{x}^{\alpha}}=0,
\end{aligned}
$$

which are the Hamel equations (Hamel (1904)).

In many concrete applications, involving, for instance, stability theory, the general form of the Lagrange-Poincaré equations given in (4.2.15) and (4.2.16) are more useful than Hamel's equations, as explained by an example in Marsden and Scheurle (1993b).

\section{Coordinate Version of Wong's Equations.}

Locally, the expression of the Lagrangian $l$ in the variables $(x, \dot{x}, \bar{v})$ is

$$
l(x, \dot{x}, \bar{v})=\frac{1}{2} \kappa_{a b} \bar{v}^{a} \bar{v}^{b}+\frac{1}{2} g_{\alpha \beta}(x) \dot{x}^{\alpha} \dot{x}^{\beta} .
$$

The local expression of the vertical Lagrange-Poincaré equation is given by (4.2.15), where

$$
p_{a}=\frac{\partial l}{\partial \bar{v}^{a}}=\kappa_{a b} \bar{v}^{b} .
$$

However, the first term on the right hand side of (4.2.15) equals

$$
p_{a} C_{d b}^{a} \bar{v}^{d}=\kappa_{a e} \bar{v}^{e} C_{d b}^{a} \bar{v}^{d} .
$$

However, bi-invariance of $\kappa$ means that

$$
\kappa_{a e} C_{d b}^{a}=\kappa_{a b} C_{e d}^{a}
$$

and so we get

$$
p_{a} C_{d b}^{a} \bar{v}^{d}=\kappa_{a e} \bar{v}^{e} C_{d b}^{a} \bar{v}^{d}=\kappa_{a b} \bar{v}^{e} C_{e d}^{a} \bar{v}^{d}=\kappa_{a b}[\bar{v}, \bar{v}]^{a}=0 .
$$


Therefore, the vertical equation becomes

$$
\frac{d p_{b}}{d t}=-p_{a} C_{d b}^{a} A_{\alpha}^{d} \dot{x}^{\alpha}
$$

The local expression of the horizontal Lagrange-Poincaré equation is given by (4.2.16), where

$$
\frac{\partial l}{\partial x^{\alpha}}=\frac{1}{2} \frac{\partial g_{\beta \gamma}(x)}{\partial x^{\alpha}} \dot{x}^{\beta} \dot{x}^{\gamma}
$$

The second term on the right hand side of (4.2.16) vanishes as in the case of the vertical equation. Therefore the horizontal Lagrange-Poincaré equation is given by

$$
\frac{d}{d t}\left(g_{\alpha \beta}(x) \dot{x}^{\beta}\right)=-p_{a} B_{\beta \alpha}^{a} \dot{x}^{\beta}+\frac{1}{2} \frac{\partial g_{\beta \gamma}(x)}{\partial x^{\alpha}} \dot{x}^{\beta} \dot{x}^{\gamma},
$$

or equivalently, with $p_{\alpha}:=g_{\alpha \beta}(x) \dot{x}^{\beta}$,

$$
\frac{d}{d t} p_{\alpha}=-p_{a} B_{\beta \alpha}^{a} \dot{x}^{\beta}-\frac{1}{2} \frac{\partial g^{\beta \gamma}}{\partial x^{\alpha}} p_{\beta} p_{\gamma},
$$

which is the second Wong equation. 


\section{5 \\ The Lie Algebra Structure on Sections of the Reduced Bundle}

The main result of this section is the establishment of a natural Lie algebra structure on the space $\Gamma(T(Q / G) \oplus \tilde{\mathfrak{g}})$ of sections of the bundle $T(Q / G) \oplus \tilde{\mathfrak{g}}$, which will be used for reduction in the next section. This quotient Lie algebra structure is defined in Definition 5.2.3 and it is computed in Theorem 5.2.4. This Lie algebra is, roughly speaking, a synthesis of the Jacobi-Lie bracket of vector fields on shape space $Q / G$ with the Lie algebra structure on the bundle $\tilde{\mathfrak{g}}$. However, as we shall see, this Lie

algebra structure involves the reduced curvature form as well.

\subsection{The Bundle $T(Q / G) \oplus \tilde{\mathfrak{g}}$ Revisited}

In this subsection, we assume that we have the following set up: a manifold $Q$, a smooth Lie group action $\rho: G \times Q \rightarrow Q$, and a connection $A$ on the principal bundle $\pi: Q \rightarrow Q / G$.

\section{Vertical and Horizontal Invariant Bundles.}

Consider the vector bundle isomorphism $\alpha_{A}: T Q / G \rightarrow T(Q / G) \oplus \tilde{\mathfrak{g}}$ defined in Lemma 2.4.2 and let $T Q=\operatorname{Hor}(T Q) \oplus \operatorname{Ver}(T Q)$ be the decomposition into horizontal and vertical parts. Since the bundles $\operatorname{Hor}(T Q)$ and $\operatorname{Ver}(T Q)$ are $G$-invariant we have

$$
T Q / G=\operatorname{Hor}(T Q) / G \oplus \operatorname{Ver}(T Q) / G .
$$

This implies $\alpha_{A}(\operatorname{Hor}(T Q) / G)=T(Q / G)$ and $\alpha_{A}(\operatorname{Ver}(T Q) / G)=\tilde{\mathfrak{g}}$. 
Definition 5.1.1. $\quad$ Let

$$
\iota_{G}(T Q): I_{G}(T Q) \rightarrow Q / G
$$

be the vector bundle whose fiber $I_{G}(T Q)_{x}=\left(\iota_{G}(T Q)\right)^{-1}(x)$ at an element $x=$ $[q]_{G} \in Q / G$ is the vector space of all $G$-invariant vector fields along $\pi^{-1}(x)$. That is,

$$
I_{G}(T Q)_{x}:=\left\{Z: \pi^{-1}(x) \rightarrow T Q \mid Z(q) \in T_{q} Q \text { for all } q \in \pi^{-1}(x) \text { and } g^{*} Z=Z\right\} .
$$

Here and in what follows we denote by $g^{*}$ the pull back of various tensorial objects by the diffeomorphism on $Q$ defined by $g \in G$ via the given $G$-action.

We also let

$$
\iota_{G}(T Q)^{V}: I_{G}^{V}(T Q) \rightarrow Q / G
$$

be the vector bundle whose fiber $I_{G}^{V}(T Q)_{x}=\left(\iota_{G}(T Q)^{V}\right)^{-1}(x)$ at an element $x=$ $[q]_{G} \in Q / G$ is the vector space of all vertical invariant vector fields on $\pi^{-1}(x)$. That is,

$$
I_{G}^{V}(T Q)_{x}:=\left\{Y \in \mathfrak{X}^{\infty}\left(\pi^{-1}(x)\right) \mid g^{*} Y=Y\right\} .
$$

We call $\iota_{G}(T Q)^{V}: I_{G}^{V}(T Q) \rightarrow Q / G$ the vertical invariant bundle.

Likewise, we define

$$
\iota_{G}(T Q)^{H}: I_{G}^{H}(T Q) \rightarrow Q / G
$$

to be the vector bundle whose fiber $I_{G}^{H}(T Q)_{x}=\left(\iota_{G}(T Q)^{H}\right)^{-1}(x)$ at an element $x=[q]_{G} \in Q / G$ is the vector space of all horizontal invariant vector fields on $Q$ along $\pi^{-1}(x)$. That is,

$$
I_{G}^{H}(T Q)_{x}:=\left\{X: \pi^{-1}(x) \rightarrow T Q \mid X(q) \in \operatorname{Hor} T_{q} Q \text { for all } q \in Q \text { and } g^{*} X=X\right\} .
$$

We call $\iota_{G}(T Q)^{H}: I_{G}^{H}(T Q) \rightarrow Q / G$ the horizontal invariant bundle.

\section{Brackets of Invariant Vector Fields.}

Recall that the Lie bracket of two $G$-invariant vector fields, say $X$ and $Y$, is again a $G$-invariant vector field. Also, if $X$ and $Y$ are both vertical $G$-invariant vector fields, that is, $X, Y \in I_{G}^{V}(T Q)$, then $[X, Y]$ is also a vertical $G$-invariant vector field, that is, $[X, Y] \in I_{G}^{V}(T Q)$.

Given $q \in Q$, let $x=[q]_{G}$, and define the diffeomorphism $\rho_{q}: G \rightarrow \pi^{-1}(x)$ by $\rho_{q}(g)=g q$. This diffeomorphism $\rho_{q}$ commutes with the action of $G$, that is, $\rho_{q}(h g)=h \rho_{q}(g)$, for all $h, g \in G$. We claim that $I_{G}^{V}(T Q)_{x}$ is isomorphic to the Lie algebra of left invariant vector fields on $G$. The Lie algebra of left invariant vector fields $\mathfrak{X}_{L}^{\infty}(G)$ is identified with $\mathfrak{g}=T_{e} G$ in the usual way. That is, to each element $\xi \in \mathfrak{g}$, we associate the element $X_{\xi} \in \mathfrak{X}_{L}^{\infty}(G)$ given by $X_{\xi}(g)=T_{e} L_{g} \cdot \xi$, where $L_{g}$ denotes the left translation map by the group element $g \in G$.

To prove the claim, we will show that the map given by the push-forward of vector fields $\rho_{q^{*}}: \mathfrak{X}_{L}^{\infty}(G) \rightarrow I_{G}^{V}(T Q)_{x}$ is a $q$-dependent Lie algebra isomorphism. 
Indeed, this map preserves the Lie algebra structure since $\rho_{q}$ commutes with the action of $G$, and it is invertible, so it is a Lie algebra isomorphism.

Notice that the tangent map of $\rho_{q}$ at $e$ is given by $T_{e} \rho_{q} \cdot \xi=\xi q$. This implies in particular that for any given $\xi \in \mathfrak{g}$, we have $\left(\rho_{q *} X_{\xi}\right)(q)=\xi q$.

On the other hand, the map $T \pi$ establishes a vector bundle isomorphism covering the identity on the base $Q$ between $I_{G}^{H}(T Q)$ and $T(Q / G)$, namely, $X \in I_{G}^{H}(T Q)_{x} \mapsto$ $T_{q} \pi(X(q)) \in T_{x}(Q / G)$ for any $q \in \pi^{-1}(x)$ (it is easy to check that this definition does not depend on the choice of $q$ in the fiber over $x$ ).

\section{The Bundles $I_{G}^{V}(T Q)$ and $\tilde{\mathfrak{g}}$ are Isomorphic.}

The next lemma shows that there is a natural vector bundle isomorphism covering the identity between these two vector bundles that also preserves the Lie bracket.

Lemma 5.1.2. The map

$$
\beta_{A}: I_{G}^{V}(T Q) \rightarrow \tilde{\mathfrak{g}}
$$

given by

$$
\beta_{A}(Y)=[q, A(Y(q))]_{G},
$$

where $Y \in I_{G}^{V}(T Q)_{x}, x \in Q / G$, and $q \in \pi^{-1}(x)$ is arbitrary, is a well defined Lie algebra bundle isomorphism. The inverse of $\beta_{A}$ is defined by the following condition: $\beta_{A}^{-1}\left([q, \xi]_{G}\right)$ is the (unique) left invariant vector field $Y$ on $\pi^{-1}(x)$ such that $Y(q)=$ $\xi q$.

Extend the domain of $\beta_{A}$ as follows: define the vector bundle isomorphism

$$
\beta_{A}: I_{G}^{H}(T Q) \oplus I_{G}^{V}(T Q) \rightarrow T(Q / G) \oplus \tilde{\mathfrak{g}}
$$

in such a way that it coincides with the isomorphism given by $T \pi$ on the summand $I_{G}^{H}(T Q)$ and with the isomorphism $\beta_{A}$ defined above on the summand $I_{G}^{V}(T Q)$.

Proof. To see that $\beta_{A}$ is well defined we simply check that for any $g \in G$ we have

$$
\begin{aligned}
{[g q, A(Y(g q))]_{G} } & =[g q, A(g Y(q))]_{G} \\
& =\left[g q, \operatorname{Ad}_{g} Y(q)\right]_{G} \\
& =[q, A(Y(q))]_{G} .
\end{aligned}
$$

It is easy to check that $\beta_{A}$ is linear on each fiber. Now we will show that $\beta_{A}$ is a Lie algebra bundle isomorphism. Let us fix $q \in Q$. Then the pull-back $\rho_{q}^{*}$ : $I_{G}^{V}(T Q)_{x} \rightarrow \mathfrak{g}$ is a Lie algebra isomorphism and we can easily check that, for all $Y \in I_{G}^{V}(T Q)_{x}$, and any $q \in \pi^{-1}(x)$ we have $\rho_{q}^{*}(Y)=A(Y(q))$. Therefore we have, for all $X, Y \in I_{G}^{V}(T Q)_{x}$,

$$
\begin{aligned}
A([X, Y](q)) & =\rho_{q}^{*}([X, Y]) \\
& =\left[\rho_{q}^{*}(X), \rho_{q}^{*}(Y)\right] \\
& =[A(X(q)), A(Y(q))] .
\end{aligned}
$$


Using this and the definition of the Lie bracket on $\tilde{\mathfrak{g}}$ (see Lemma 2.3.5) we can write

$$
\begin{aligned}
\beta_{A}([X, Y]) & =[q, A([X, Y](q))]_{G} \\
& =[q,[A(X(q)), A(Y(q))]]_{G} \\
& =\left[\beta_{A}(X), \beta_{A}(Y)\right] .
\end{aligned}
$$

It is clear that $\beta_{A}^{-1}$ is given by the rule described in the statement since $A(Y(q))=\xi$ if $Y(q)=\xi q$. The rest of the proof, namely, to show that the extended $\beta_{A}$ is a vector bundle isomorphism is obvious.

\subsection{The Lie Algebra of Sections of $T(Q / G) \oplus \tilde{\mathfrak{g}}$}

\section{Quotient Vector Bundles.}

We begin with some preliminaries concerning quotient vector bundles with some additional structures.

Let $\tau: V \rightarrow Q$ be a given vector bundle and let $\rho: G \times V \rightarrow V$ denote a given $G$-action on $V$ (see $\S 2.1)$.

Recall that, by definition, the action $\rho$ is a vector bundle action if for each $g \in G$ the map $\rho_{g}: V \rightarrow V$ is a vector bundle isomorphism. This implies, in particular, that there is an action $\rho_{0}: G \times Q \rightarrow Q$ such that $\tau: V \rightarrow Q$ is equivariant and for each $q \in Q$ the restriction of $\rho_{g}$ to $\tau^{-1}(q)$ is a linear isomorphism $\rho_{g}: \tau^{-1}(q) \rightarrow \tau^{-1}\left(\rho_{0 g}(q)\right)$. We will often use the simpler notation $g v$ and $g q$ instead of $\rho_{g}(v)$ and $\rho_{0 g}(q)$ respectively. As mentioned in $\S 2.1$, throughout this work we will assume that the action $\rho_{0}$ of $G$ on $Q$ is free and, moreover, that relative to this action $Q \rightarrow Q / G$ is a principal $G$-bundle. Then $V$ is also a principal $G$-bundle. Although this assumption is not strictly needed for the validity of some properties, we will still take it for granted, to simplify the exposition. An immediate consequence of this is that the quotient $V / G$ carries a naturally defined vector bundle structure over the base $Q / G$.

More precisely, we have the following lemma, whose proof, which is standard, is included for the sake of completeness.

Lemma 5.2.1. The quotient $V / G$ carries a naturally defined vector bundle structure over the base $Q / G$, say $\tau / G: V / G \rightarrow Q / G$, where $(\tau / G)\left([v]_{G}\right)$ is defined by $(\tau / G)\left([v]_{G}\right)=[\tau v]_{G}$.

The projection $\pi_{G}(V): V \rightarrow V / G$ is a surjective vector bundle homomorphism covering $\pi$, and the restriction $\pi_{G}(V) \mid \tau^{-1}(q): \tau^{-1}(q) \rightarrow(\tau / G)^{-1}\left([q]_{G}\right)$ is a linear isomorphism for each $q \in Q$. In addition, $\pi_{G}(V)\left|\tau^{-1}(q) \circ g^{-1}=\pi_{G}(V)\right| \tau^{-1}(g q)$ for all $q \in Q$ and $g \in G$.

Assume that $\tau^{\prime}: V^{\prime} \rightarrow Q^{\prime}$ is another vector bundle and that there is a vector bundle action $G \times V^{\prime} \rightarrow V^{\prime}$. Let $f: V \rightarrow V^{\prime}$ be an equivariant vector bundle map. Then the naturally induced quotient map $[f]_{G}: V / G \rightarrow V^{\prime} / G$ is a vector bundle map. If $f$ is a vector bundle isomorphism, so is $[f]_{G}$. 
Proof. First we show that $\tau / G$ is well defined, namely, for any $g \in G$ and any $v \in V$ we have $\tau / G\left([g v]_{G}\right)=[\tau g v]_{G}=[g \tau v]_{G}=[\tau v]_{G}$. Next we define the vector space structure on each fiber $(\tau / G)^{-1}\left([q]_{G}\right)$. Let $\left[v_{i}\right]_{G} \in V / G$, for $i=1,2$, be such that $\tau / G\left[v_{1}\right]_{G}=\tau / G\left[v_{2}\right]_{G}=[q]_{G}$, for some $q \in Q$. This implies that there are $g_{i} \in G$ such that $g_{i} \tau v_{i}=q$ for $i=1,2$. The $g_{i}$ are uniquely determined for a fixed $q$, as before, because the action $\rho_{0}$ is free. Define $\left[v_{1}\right]_{G}+\left[v_{2}\right]_{G}=\left[g_{1} v_{1}+g_{2} v_{2}\right]_{G}$. We must show that this gives a well defined additive structure. Elements of $V$ equivalent to $v_{i}$ are of the type $h_{i} v_{i}$ with $h_{i} \in G$ for $i=1$,2. For given $h_{i} v_{i}$ with $h_{i} \in G, i=1,2$, the only elements $d_{i} \in G$ such that $d_{i} \tau h_{i} v_{i}=q$ are $d_{i}=g_{i} h_{i}^{-1}$, $i=1,2$. Then our definition gives

$$
\begin{aligned}
{\left[h_{1} v_{1}\right]_{G}+\left[h_{2} v_{2}\right]_{G}=\left[d_{1} h_{1} v_{1}+d_{2} h_{2} v_{2}\right]_{G} } & =\left[g_{1} v_{1}+g_{2} v_{2}\right]_{G} \\
& =\left[v_{1}\right]_{G}+\left[v_{2}\right]_{G}
\end{aligned}
$$

This shows that the definition does not depend on the choice of the representative in the class $\left[v_{i}\right]_{G}, i=1,2$, for a given $q$. Now, if we choose an arbitrary element, say $h q \in[q]_{G}$, then the only elements $d_{i} \in G$ such that $d_{i} \tau v_{i}=h q$ are $d_{i}=h g_{i}$, for $i=1,2$. We obtain then

$$
\begin{aligned}
{\left[v_{1}\right]_{G}+\left[v_{2}\right]_{G} } & =\left[h g_{1} v_{1}+h g_{2} v_{2}\right]_{G} \\
& =\left[h\left(g_{1} v_{1}+g_{2} v_{2}\right)\right]_{G} \\
& =\left[g_{1} v_{1}+g_{2} v_{2}\right]_{G}
\end{aligned}
$$

as before.

We can define $\lambda[v]_{G}=[\lambda v]_{G}$ and check that it is well defined in a similar way. To finish the proof, it only remains to show that the restriction of $\pi_{G}(V)$ to each fiber, $\pi_{G}(V): \tau^{-1}(q) \rightarrow(\tau / G)^{-1}\left([q]_{G}\right)$, is a linear isomorphism. This can be easily established using the definition of the linear structure on $(\tau / G)^{-1}\left([q]_{G}\right)$. We omit the rest of the proof, which can also be easily performed using standard techniques.

\section{Spaces of Sections.}

For a vector bundle $\tau: V \rightarrow Q$, the vector space of sections of $V$ is denoted by $\Gamma(V)$, which is also a $C^{\infty}(Q)$-module. Let $f: V_{1} \rightarrow V_{2}$ be a vector bundle homomorphism where $\tau^{i}: V_{i} \rightarrow Q_{i}$ are vector bundles for $i=1,2$. This implies, in particular, that there is a map $f_{0}: Q_{1} \rightarrow Q_{2}$ such that, for each $q \in Q_{1}$, the restriction $f:\left(\tau^{1}\right)^{-1}(q) \rightarrow\left(\tau^{2}\right)^{-1}\left(f_{0}(q)\right)$ is linear. Assume, in addition, that this restriction is a linear isomorphism. Then $f$ induces a linear map $f^{*}: \Gamma\left(V_{2}\right) \rightarrow \Gamma\left(V_{1}\right)$ defined by $f^{*}(s)(q)=f^{-1}\left(s\left(f_{0}(q)\right)\right)$, where $f^{-1}$ is the inverse of the restriction of $f$ to $\left(\tau^{2}\right)^{-1}(q)$. The properties $(f \circ h)^{*}=h^{*} \circ f^{*}$ and $\operatorname{id}_{V}^{*}=\operatorname{id}_{\Gamma(V)}$, where both $f$ and $h$ satisfy the condition that their restriction to each fiber is a linear isomorphism, can be easily checked. It follows, in particular, that if $f$ is an isomorphism, then $f^{-1 *}=f^{*-1}$. In this case we write $f^{-1 *}=f_{*}$.

Sections $s_{i} \in \Gamma\left(V_{i}\right), i=1,2$, are said to be $f$-related if for all $q \in Q_{1}$ we have $f\left(s_{1}(q)\right)=s_{2}\left(f_{0}(q)\right)$. We can easily show that if for each $q \in Q_{1}$ the restriction 
$f:\left(\tau^{1}\right)^{-1}(q) \rightarrow\left(\tau^{2}\right)^{-1}\left(f_{0}(q)\right)$ is a linear isomorphism as before, then for any given $s_{2} \in \Gamma\left(V_{2}\right)$, the section $s_{1}=f^{*}\left(s_{2}\right)$ is the only one in $\Gamma\left(V_{1}\right)$ which is $f$-related to $s_{2}$.

If $G \times V \rightarrow V$ is a vector bundle action on $\tau: V \rightarrow Q$, a section $s: Q \rightarrow V$ is called an invariant section if for all $g \in G$ and all $q \in Q$ we have $g s(q)=s(g q)$. The set $\Gamma^{G}(V)$ of invariant sections of $V$ is a subspace of $\Gamma(V)$.

Lemma 5.2.2. Let $G \times V \rightarrow V$ be a vector bundle action and let $\pi_{G}(V): V \rightarrow$ $V / G$ be the vector bundle homomorphism described in lemma 5.2.1. Then $\left(\pi_{G}(V)\right)^{*}$ : $\Gamma(V / G) \rightarrow \Gamma^{G}(V)$ is a linear isomorphism.

Proof. Let $\bar{s} \in \Gamma(V / G)$. Then for each $q \in Q$ and each $g \in G$, using the fact that the restriction of $\pi_{G}(V)$ to each fiber is an isomorphism, we have

$$
\begin{aligned}
\pi_{G}(V)^{*} \bar{s}(g q) & =\pi_{G}(V)^{-1}\left(\bar{s}\left([g q]_{G}\right)\right) \\
& =g\left(\pi_{G}(V)^{-1}\left(\bar{s}\left([q]_{G}\right)\right)\right) \\
& =g \pi_{G}(V)^{*} \bar{s}(q),
\end{aligned}
$$

where in the first equation $\pi_{G}(V)$ is restricted to $\tau^{-1}(g q)$ and in the second $\pi_{G}(V)$ is restricted to $\tau^{-1}(q)$. The second equality is then an easy consequence of the definition of a vector bundle action and, also, the definition of the quotient vector bundle (see Lemma 5.2.1). Thus, we have shown that $\pi_{G}(V)^{*}$ is an injective map into $\Gamma^{G}(V)$. Now let $s \in \Gamma^{G}(V)$. Define $\bar{s} \in \Gamma(V / G)$ by $\bar{s}\left([q]_{G}\right)=[s(q)]_{G}$. The element $\bar{s}$ is well defined because, since $s$ is invariant, for any $g \in G$ and any $q \in Q$ we have $[s(g q)]_{G}=[g s(q)]_{G}=[s(q)]_{G}$. We can easily check that $s=\pi_{G}(V)^{*} \bar{s}$ which finishes the proof.

\section{Quotient Lie Algebras.}

If $v \in \Gamma^{G}(V)$ we will denote $[v]_{G}$ or, sometimes, $\bar{v}$ the corresponding section of $\Gamma(V / G)$ via the isomorphism $\pi_{G}(V)^{*}$ of the previous lemma. Let $\rho$ be a vector bundle action of the Lie group $G$ on the vector bundle $\tau: V \rightarrow Q$. Then we obtain a representation $\rho_{*}: G \times \Gamma(V) \rightarrow \Gamma(V)$ given by the operation $(g, s) \in G \times \Gamma(V) \mapsto$ $\rho_{g *} s \in \Gamma(V)$. We will often write simply $g_{*} s$ instead of $\rho_{g *} s$. It is clear that $\Gamma^{G}(V)$ is an invariant subspace of $\Gamma(V)$. Now assume that there is a Lie algebra structure on $\Gamma(V)$ which is invariant under the action $\rho_{*}$, that is, $g_{*}\left[s_{1}, s_{2}\right]=\left[g_{*} s_{1}, g_{*} s_{2}\right]$ for all $s_{i} \in \Gamma(V), i=1,2$, and all $g \in G$. In particular, $\Gamma^{G}(V)$ is a Lie subalgebra of $\Gamma(V)$.

Since $\pi_{G}(V)^{*}: \Gamma(V / G) \rightarrow \Gamma^{G}(V)$ is a linear isomorphism we can define a Lie algebra structure on $\Gamma(V / G)$ in the following way.

Definition 5.2.3. Assume that the space of sections $\Gamma(V)$ of the vector bundle $\tau: V \rightarrow Q$ has a Lie algebra structure. The quotient Lie algebra structure on $\Gamma(V / G)$ is defined by

$$
\left[\bar{s}_{1}, \bar{s}_{2}\right]=\left(\pi_{G}(V)^{*}\right)^{-1}\left[\pi_{G}(V)^{*} \bar{s}_{1}, \pi_{G}(V)^{*} \bar{s}_{2}\right] .
$$


The most important case of the situation described above is the case of the vector bundle $T Q$ on which $G$ acts by the tangent lift of the action of $G$ on $Q$ and the Lie bracket on $\Gamma(T Q) \equiv \mathfrak{X}^{\infty}(Q)$ is the usual Lie bracket of vector fields. According to the previous results we obtain a quotient Lie algebra structure on $\Gamma(T Q / G)$.

Let us denote $\pi \equiv \pi_{G}(Q)$ the natural projection of the principal bundle $\pi: Q \rightarrow$ $Q / G$, for simplicity. Recall that for each choice of a principal connection $A$ on $Q$ we have vector bundle isomorphisms

$$
\alpha_{A}: T Q / G \rightarrow T(Q / G) \oplus \tilde{\mathfrak{g}}
$$

and

$$
\beta_{A}: I_{G}^{H}(T Q) \oplus I_{G}^{V}(T Q) \rightarrow T(Q / G) \oplus \tilde{\mathfrak{g}} .
$$

The linear isomorphism $\alpha_{A}^{*}$ between the corresponding spaces of sections defines a Lie algebra structure on $\Gamma(T(Q / G) \oplus \tilde{\mathfrak{g}})$ by declaring it to be a Lie algebra isomorphism. In order to calculate this Lie bracket on $\Gamma(T(Q / G) \oplus \tilde{\mathfrak{g}})$ we shall make use of the equivalent condition that $\beta_{A}^{*}$ is a Lie algebra isomorphism. We do this in the next theorem.

The map $T \pi$ induces a well defined isomorphism between $\Gamma\left(I_{G}^{H}(T Q)\right)$ and $\mathfrak{X}^{\infty}(Q / G)$. More precisely, for any $X \in \Gamma\left(I_{G}^{H}(T Q)\right)$ the vector field $\pi_{*} X \in \mathfrak{X}^{\infty}(Q / G)$ given by $\pi_{*} X(x)=T \pi X(q)$, where $q \in \pi^{-1}(x)$ is arbitrary, is well defined. Also, if $X, Y \in \Gamma\left(I_{G}^{H}(T Q)\right)$ then $\pi_{*}[X, Y]=\left[\pi_{*} X, \pi_{*} Y\right]$ since the bracket operation preserves $\pi$-relatedness of vector fields. Observe that the inverse of $\pi_{*}: \Gamma\left(I_{G}^{H}(T Q)\right) \rightarrow$ $\mathfrak{X}^{\infty}(Q / G)$ is given by the horizontal lift of vector fields, ${ }^{h}: \mathfrak{X}^{\infty}(Q / G) \rightarrow \Gamma\left(I_{G}^{H}(T Q)\right)$, which also coincides with the restriction of $\beta_{A}^{*}$ to $\mathfrak{X}^{\infty}(Q / G)$. Also, we recall that if $X, Y \in \Gamma\left(I_{G}^{H}(T Q)\right)$ then $A([X, Y])=-B(X, Y)$ where $B$ is the curvature of $A$. Finally we remark that, from what we have said before, we can deduce that there are natural identifications

$$
\Gamma^{G}(T Q) \equiv \Gamma(T Q / G) \equiv \Gamma\left(I_{G}^{H}(T Q)\right) \oplus \Gamma\left(I_{G}^{V}(T Q)\right),
$$

where the last identification involves the choice of a connection.

\section{Calculation of the Lie Algebra Structure.}

The main result of this section is a formula, given in the next theorem, for the Lie bracket on the Lie algebra $\Gamma(T(Q / G) \oplus \tilde{\mathfrak{g}}) \equiv \mathfrak{X}^{\infty}(Q / G) \oplus \Gamma(\tilde{\mathfrak{g}})$ which involves the Lie bracket on $\tilde{\mathfrak{g}}$, the connection $\tilde{\nabla}^{A}$ on $\tilde{\mathfrak{g}}$, and the $\tilde{\mathfrak{g}}$-valued curvature $\tilde{B}^{A}$.

Theorem 5.2.4. Let $X_{i} \oplus \bar{\xi}_{i} \in \Gamma(T(Q / G) \oplus \tilde{\mathfrak{g}}), i=1,2$, be given sections of $T(Q / G) \oplus \tilde{\mathfrak{g}}$. Then

$$
\left[X_{1} \oplus \bar{\xi}_{1}, X_{2} \oplus \bar{\xi}_{2}\right]=\left[X_{1}, X_{2}\right] \oplus \tilde{\nabla}_{X_{1}}^{A} \bar{\xi}_{2}-\tilde{\nabla}_{X_{2}}^{A} \bar{\xi}_{1}-\tilde{B}^{A}\left(X_{1}, X_{2}\right)+\left[\bar{\xi}_{1}, \bar{\xi}_{2}\right]
$$

A remark is in order to avoid any confusion in the interpretation of the bracket notation in the preceding and in several other formulas. Namely, the bracket on the left-hand-side is the bracket in $\Gamma(T(Q / G) \oplus \tilde{\mathfrak{g}})$ given in Definition 5.2.3, while the 
bracket that appears immediately before the sign $\oplus$ is the usual bracket of vector fields. This caution is needed to avoid the apparently contradictory statement

$$
\left[X_{1}, X_{2}\right] \equiv\left[X_{1} \oplus 0, X_{2} \oplus 0\right]=\left[X_{1}, X_{2}\right] \oplus-\tilde{B}^{A}\left(X_{1}, X_{2}\right) .
$$

Proof of the Theorem.. Let $X_{i} \oplus \bar{\xi}_{i} \in \Gamma(T(Q / G) \oplus \tilde{\mathfrak{g}}), i=1,2$. Then there are elements $Y_{i} \in \Gamma\left(I_{G}^{V}(T Q)\right)$ and $X_{i}^{h} \in \Gamma\left(I_{G}^{H}(T Q)\right)$, where $X_{i}^{h}$ is the horizontal lift of $X_{i} \in \mathfrak{X}^{\infty}(Q / G)$, such that $\beta_{A}^{*} \bar{\xi}_{i}=Y_{i}$ and $\beta_{A}^{*} X_{i}=X_{i}^{h}$ for $i=1,2$. According to the definition of the bracket in $\Gamma(T(Q / G) \oplus \tilde{\mathfrak{g}})$ given before the theorem, we have

$$
\left[X_{1} \oplus \bar{\xi}_{1}, X_{2} \oplus \overline{\xi_{2}}\right]=\beta_{A *}\left[X_{1}^{h}+Y_{1}, X_{2}^{h}+Y_{2}\right] .
$$

For any $q \in Q$ let $x=\pi(q)=[q]_{G}$. By (2.2.4) we have

$$
\left[X_{1}^{h}, X_{2}^{h}\right](q)=\left[X_{1}, X_{2}\right]^{h}(q)-B\left(X_{1}^{h}(q), X_{2}^{h}(q)\right) q .
$$

Applying $\beta_{A *}$ to this identity and evaluating the result at $x=[q]_{G}$, we obtain, in the Lie algebra $\Gamma(T(Q / G) \oplus \tilde{\mathfrak{g}})$,

$$
\begin{aligned}
{\left[X_{1}, X_{2}\right](x) } & =\left[X_{1}, X_{2}\right](x) \oplus\left[q,-B\left(X_{1}^{h}(q), X_{2}^{h}(q)\right)\right]_{G} \\
& =\left[X_{1}, X_{2}\right](x) \oplus-\tilde{B}^{A}(x)\left(X_{1}, X_{2}\right) ;
\end{aligned}
$$

again, the bracket $\left[X_{1}, X_{2}\right]$ on the left hand side is that in $\Gamma(T(Q / G) \oplus \tilde{\mathfrak{g}})$, whereas the same notation on the right hand side signifies the usual Lie bracket of vector fields. Using Lemma 5.1.2 we can deduce that for any $x \in Q / G$,

$$
\beta_{A *}\left[Y_{1}, Y_{2}\right](x)=\left[\bar{\xi}_{1}, \bar{\xi}_{2}\right](x) .
$$

Thus, we are left with the computation of the terms $\left[X_{1}, \overline{\xi_{2}}\right]=\beta_{A *}\left[X_{1}^{h}, Y_{2}\right]$ and $\left[X_{2}, \bar{\xi}_{1}\right]=\beta_{A *}\left[X_{2}^{h}, Y_{1}\right]$. For this, we first recall that, since $X_{1}^{h}$ is an invariant horizontal vector field, its flow $X_{1 t}^{h}$, which is the horizontal lift of the flow $X_{1 t}$ of $X_{1}$, maps a point $q \in \pi^{-1}(x)$ to a point $X_{1 t}^{h}(q) \in \pi^{-1}\left(X_{1 t}(x)\right)$. Since $X_{1}^{h}$ and $X_{1}$ are $\pi$-related, we have $\pi \circ X_{1 t}^{h}=X_{1 t} \circ \pi$, and thus, for any vertical vector field $Y$ on $Q$, the pull back $X_{1 t}^{h *} Y$ is also vertical. Therefore the Lie bracket

$$
\left[X_{1}^{h}, Y\right]=\left.\frac{d}{d t} X_{1 t}^{h *} Y\right|_{t=0}
$$

is also a vertical vector field. Using this, we see that for any $q \in Q$ we have

$$
\left[X_{1}^{h}, Y_{2}\right](q)=A\left(\left[X_{1}^{h}, Y_{2}\right](q)\right) q .
$$

The $G$-invariance of $X_{1}^{h}$ and $Y_{2}$, immediately implies the $G$-invariance of $\left[X_{1}^{h}, Y_{2}\right]$. Thus $\left[X_{1}^{h}, Y_{2}\right]$ is a vertical $G$-invariant vector field.

Second, we need to calculate $\beta_{A *}\left[X_{1}^{h}, Y_{2}\right]$. For any $x \in Q / G$ and any $q \in \pi^{-1}(x)$, we have

$$
\beta_{A *}\left[X_{1}^{h}, Y_{2}\right](x)=\left[q, A\left(\left[X_{1}^{h}, Y_{2}\right](q)\right)\right]_{G} .
$$


Cartan's structure equations give

$$
\mathbf{d} A\left(X_{1}^{h}, Y_{2}\right)=\left[A\left(X_{1}^{h}\right), A\left(Y_{2}\right)\right]+B\left(X_{1}^{h}, Y_{2}\right) .
$$

Since $A\left(X_{1}^{h}\right)=0$ (because $X_{1}^{h}$ is horizontal) and $B\left(X_{1}^{h}, Y_{2}\right)=0$ (because $Y_{2}$ is vertical), we obtain $\mathbf{d} A\left(X_{1}^{h}, Y_{2}\right)=0$. On the other hand we have the formula

$$
\mathbf{d} A\left(X_{1}^{h}, Y_{2}\right)=X_{1}^{h}\left[A\left(Y_{2}\right)\right]-Y_{2}\left[A\left(X_{1}^{h}\right)\right]-A\left(\left[X_{1}^{h}, Y_{2}\right]\right) .
$$

We conclude that $A\left(\left[X_{1}^{h}, Y_{2}\right]\right)=X_{1}^{h}\left[A\left(Y_{2}\right)\right]$. This shows, in particular, that at a given point $q \in Q, A\left(\left[X_{1}^{h}, Y_{2}\right](q)\right)$ only depends on $X_{1}^{h}(q)$ and not on the behavior of $X_{1}^{h}$ in a neighborhood of $q$. Now it is clear that, for any $q \in Q$, we have

$$
\left(X_{1}^{h}\left[A\left(Y_{2}\right)\right]\right)(q)=\left.\frac{d}{d t} A\left(Y_{2}\left(X_{1 t}^{h}(q)\right)\right)\right|_{t=0} .
$$

Let $q \in Q$ and $x=\pi(q)$; thus $X_{1 t}(x)=\pi\left(X_{1 t}^{h}(q)\right)$, for all $t$. Let $\xi_{2}(t)=A\left(Y_{2}\left(X_{1 t}^{h}(q)\right)\right)$ for all $t$. Lemma 5.1.2 implies that $\bar{\xi}_{2}(x(t))=\left[X_{1 t}^{h}(q), \xi_{2}(t)\right]_{G}$ for all $t$ and therefore

$$
\tilde{\nabla}_{X_{1}(x)}^{A} \bar{\xi}_{2}(x)=\left.\frac{D}{D t}\left[X_{1 t}^{h}(q), \xi_{2}(t)\right]_{G}\right|_{t=0} .
$$

Now using Lemma 2.3.4 to calculate the covariant derivative of the curve $\left[X_{1 t}^{h}(q), \xi(t)\right]_{G} \in$ $\tilde{\mathfrak{g}}$ we obtain

$$
\left.\frac{D}{D t}\left[X_{1 t}^{h}(q), \xi_{2}(t)\right]_{G}\right|_{t=0}=\left[q, \dot{\xi}_{2}(0)\right]_{G} .
$$

But $\dot{\xi}_{2}(0)=\left(X_{1}^{h} A\left(Y_{2}\right)\right)(q)$ which implies $\dot{\xi}_{2}(0)=A\left(\left[X_{1}^{h}, Y_{2}\right](q)\right)$. We conclude that

$$
\tilde{\nabla}_{X_{1}}^{A} \bar{\xi}_{2}=\beta_{A *}\left[X_{1}^{h}, Y_{2}\right]
$$

Analogously, we have $\tilde{\nabla}_{X_{2}}^{A} \bar{\xi}_{1}=\beta_{A *}\left[X_{2}^{h}, Y_{1}\right]$.

Collecting these results, we obtain

$$
\left[X_{1} \oplus \bar{\xi}_{1}, X_{2} \oplus \bar{\xi}_{2}\right]=\left[X_{1}, X_{2}\right] \oplus \tilde{\nabla}_{X_{1}}^{A} \bar{\xi}_{2}-\tilde{\nabla}_{X_{2}}^{A} \bar{\xi}_{1}-\tilde{B}\left(X_{1}, X_{2}\right)+\left[\bar{\xi}_{1}, \bar{\xi}_{2}\right],
$$

as desired.

The following corollary is a consequence of Theorem 5.2.4.

Corollary 5.2.5. Let $p_{Q / G}: T(Q / G) \oplus \tilde{\mathfrak{g}} \rightarrow T(Q / G)$ and $p_{\mathfrak{g}}: T(Q / G) \oplus \tilde{\mathfrak{g}} \rightarrow \tilde{\mathfrak{g}}$ be the natural projections. Then the induced maps $p_{(Q / G) *}: \Gamma(T(Q / G) \oplus \tilde{\mathfrak{g}}) \rightarrow$ $\Gamma(T(Q / G))$ given by $p_{(Q / G) *}(X \oplus \bar{\xi})=X$ and $p_{\mathfrak{\mathfrak { g }}}^{*}: \Gamma(\tilde{\mathfrak{g}}) \rightarrow \Gamma(T(Q / G) \oplus \tilde{\mathfrak{g}})$ given by $p_{\mathfrak{\mathfrak { g }}}^{*}(\bar{\xi})=0 \oplus \bar{\xi}$ are Lie algebra homomorphisms. The Lie algebra structure on $\Gamma(\tilde{\mathfrak{g}})$ is defined pointwise, that is, for given $\bar{\xi}, \bar{\eta} \in \Gamma(\tilde{\mathfrak{g}})$ we have, $[\bar{\xi}, \bar{\eta}](x)=[\bar{\xi}(x), \bar{\eta}(x)]$ for all $x \in Q / G$. 


\section{6}

\section{Reduced Tangent Bundles}

The results of the preceding sections may be viewed as a geometrized and intrinsic way of writing the results of Cendra, Ibort and Marsden (1987) and of Marsden and Scheurle (1993b). Next we turn to our main task: find a context in which the Lagrangian reduction process can be iterated. In other words, we develop a framework in which the objects are stable under Lagrangian reduction. These objects will be called reduced tangent bundles.

\section{Lagrange-Poincaré Bundles and Reduced Tangent Bundles.}

We begin by describing the geometric objects on which reduced Lagrangians are naturally defined. They form a category of vector bundles denoted $\mathfrak{L P}$, objects of which will be called Lagrange-Poincaré bundles. Important special LagrangePoincaré bundles are the reduced tangent bundles. These form a subcategory, denoted $\mathfrak{R T}$, which is the smallest subcategory that contains the tangent bundles and which remains stable under reduction; each bundle carries some additional structure needed to describe the reduction of given Lagrangians defined on them and the corresponding variational principles.

For example, objects of $\mathfrak{R T}$ are vector bundles of the type $T(Q / G) \oplus \tilde{\mathfrak{g}}$ where, as we have seen, $\tilde{\mathfrak{g}}$ is a Lie algebra bundle, there is a covariant derivative $\tilde{\nabla}^{A}$ on $\tilde{\mathfrak{g}}$, a $\tilde{\mathfrak{g}}$-valued 2-form $\tilde{B}$, and the space of sections of $T(Q / G) \oplus \tilde{\mathfrak{g}}$ admits a Lie algebra structure which is related to $\tilde{\nabla}^{A}, \tilde{B}$, and the Lie algebra structure on the fibers of $\tilde{\mathfrak{g}}$ by the formula of Theorem 5.2.4.

In this section we show how to reduce further these kinds of objects. They are, in a sense, special cases of Lie algebroids (see Mackenzie (1987), Courant (1990), Weinstein [1996, 1998] and Cannas da Silva and Weinstein (1999)) although we 
consider some extra structure on them. We also recall (see Weinstein (1996) and Marsden et al. (2000)) that these notions are very useful in discrete Lagrangian mechanics. Further exploration of the link between our work, that of groupoids and algebroids, as well as noncommutative differential geometry would of course be very interesting.

Below we will show that the reduction process can be performed by stages and we will also write explicit expressions for Lagrangians and variational principles reduced by stages.

Even though the most important objects of $\mathfrak{L P}$ seem to be those of $\mathfrak{R T}$, it is natural to first deal with the larger category $\mathfrak{L P}$ rather than the category $\mathfrak{R T}$.

The objects in the category $\mathfrak{L P}$ of Lagrange-Poincaré bundles are vector bundles of the type $T Q \oplus V$ where $V$ is a Lie algebra bundle over the base $Q$ and which carry

1. a covariant derivative $D$ on $V$,

2. a $V$-valued 2-form on $Q$, and

3. a Lie algebra structure on $\Gamma(T Q \oplus V)$ that satisfies an equality formally similar to the formula of Theorem 5.2.4.

We shall make this more explicit and describe the mappings in this category shortly.

We will show that the category $\mathfrak{R T}$ is strictly contained in the category $\mathfrak{L P}$. Moreover, the simplest way of describing $\mathfrak{R T}$ is by defining it as the smallest subcategory of $\mathfrak{L P}$ that contains the tangent bundles $T Q$. The special importance of the category $\mathfrak{R T}$ is that each of its objects is a reduced tangent bundle, after some number of reduction by stages; in addition, reduced versions of Euler-Lagrange equations corresponding to invariant Lagrangians on tangent bundles can be written in terms of these reduced tangent bundles.

It is important to remark at this point that we can obtain a generalization of all this, that is, a category bigger than $\mathfrak{L P}$, which contains in particular vector bundles which are some subbundles of bundles of $\mathfrak{L P}$, to describe reduction in nonholonomic mechanics. This, as well as other interesting topics like a local study of objects of $\mathfrak{L P}$, will be the purpose of future work.

An important result in this section is an explicit expression for the reduction by stages of the Lie algebra of sections of bundles which are objects of $\mathfrak{L P}$. This, together with the results of $\S 5.2$, will be related in $\S 8$ to the Poisson bracket on the dual bundles of the bundles which are objects of $\mathfrak{L P}$. These dual bundles carry a Poisson bracket induced by the structure of the objects of $\mathfrak{L P}$. Therefore, by duality, we obtain a direct link to the topic of Poisson reduction by stages of at least some important examples of Poisson manifolds. The special case of duals of elements of $\mathfrak{R T}$ gives cotangent Poisson reduction (see Montgomery, Marsden and Ratiu (1984) and Montgomery (1986)). A generalization of all this for more general Poisson manifolds is tied to the consideration of a category bigger than $\mathfrak{L P}$ as indicated above, and will be also the purpose of future work. 


\subsection{The Geometry of Lagrange-Poincaré Bundles}

We will need to fix some notation for maps induced on quotients. Recall that if a Lie group $G$ acts on the manifolds $E$ and $F$ and $f: E \rightarrow F$ is a $G$-equivariant map, then there is a natural quotient map $f / G: E / G \rightarrow F / G$, defined by

$$
f / G\left([a]_{G}\right)=[f(a)]_{G}
$$

\section{Lagrange-Poincaré Bundles.}

We now give the details of the definition of a Lagrange-Poincaré bundle.

Definition 6.1.1. The category $\mathfrak{L P}$ of Lagrange-Poincaré bundles is defined as follows:

(A) The objects of $\mathfrak{L P}$ are vector bundles which are Whitney sums of the form $\tau_{Q} \oplus \tau: T Q \oplus V \rightarrow Q$ where $\tau_{Q}: T Q \rightarrow Q$ is the tangent bundle of a manifold $Q$ and $\tau: V \rightarrow Q$ is a vector bundle, together with some extra structure given by:

(a) a Lie algebra structure on each fiber of $V$, in such a way that $V$ is a Lie algebra bundle; the Lie bracket of given elements $v_{1}, v_{2} \in \tau^{-1}(q)$ is denoted by $\left[v_{1}, v_{2}\right]$;

(b) a $V$-valued 2 -form $\omega$ on $Q$;

(c) a covariant derivative $D / D$ t for curves in $V$, related in the standard way to a connection $\nabla$ on $V$, namely, if $v(t)$ is any curve in $V$, consider the curve $q(t)=\tau(v(t))$ on $Q$, and let

$$
T_{t}^{t+s}: \tau^{-1} q(t+s) \rightarrow \tau^{-1} q(t)
$$

be the parallel transport along $q(t)$ defined by $\nabla$; then

$$
\frac{D v(t)}{D t}=\left.\frac{d}{d s} T_{t}^{t+s} v(t+s)\right|_{s=0} ;
$$

(d) a Lie bracket operation defined on sections $X \oplus u \in \Gamma(T Q \oplus V)$ which is defined by

$$
\left[X_{1} \oplus u_{1}, X_{2} \oplus u_{2}\right]=\left[X_{1}, X_{2}\right] \oplus \nabla_{X 1} u_{2}-\nabla_{X 2} u_{1}-\omega\left(X_{1}, X_{2}\right)+\left[u_{1}, u_{2}\right]
$$

(B) Let $T Q_{i} \oplus V_{i}, i=1,2$, be two Lagrange-Poincaré bundles with structures $[,]_{i}, \omega_{i}, \nabla_{i}$ on $\tau^{i}: V_{i} \rightarrow Q_{i}, i=1,2$. Let $D_{i} / D_{i}$ t denote the covariant derivative along a curve in $Q_{i}$ defined by $\nabla_{i}$ on $V_{i}, i=1,2$. A morphism $f: T Q_{1} \oplus V_{1} \rightarrow T Q_{2} \oplus V_{2}$ between Lagrange-Poincaré bundles is a vector bundle map covering $f_{0}: Q_{1} \rightarrow Q_{2}$ that satisfies the following conditions:

(a) $f\left(T Q_{1}\right) \subset T Q_{2}$ and, moreover, $f \mid T Q_{1}=T f_{0}$; 
(b) $f\left(V_{1}\right) \subset V_{2}$ and the restricted vector bundle map $f \mid V_{1}$ commutes with the structures on $V_{i}$ given by $[,]_{i}, \omega_{i}, \nabla_{i}$, which means that for given $u, u^{\prime} \in\left(\tau^{1}\right)^{-1}(q), X, X^{\prime} \in \tau_{Q_{1}}^{-1}(q)$, and a given curve $v(t)$ in $V_{1}$, the following conditions are satisfied

$$
\begin{gathered}
f\left(\left[u, u^{\prime}\right]_{1}\right)=\left[f(u), f\left(u^{\prime}\right)\right]_{2}, \\
f\left(\omega_{1}\left(X, X^{\prime}\right)\right)=\omega_{2}\left(f(X), f\left(X^{\prime}\right)\right),
\end{gathered}
$$

and

$$
f\left(\frac{D_{1} v(t)}{D_{1} t}\right)=\frac{D_{2} f(v(t))}{D_{2} t} .
$$

Bundles of the type $T(Q / G) \oplus \tilde{\mathfrak{g}}$ as considered in previous sections are important examples of elements of $\mathfrak{L} \mathfrak{P}$.

\section{Projections and Injections.}

The following lemma is an easy consequence of the previous definition.

Lemma 6.1.2. Let $T Q \oplus V$ be an object of $\mathfrak{L P}$ and let $\nabla, \omega,[$,$] be the structure$ on $V$. Then the following statements hold.

(i) The maps $\Gamma(T Q \oplus V) \rightarrow \Gamma(T Q)$ given by $X \oplus v \mapsto X$ and $\Gamma(V) \rightarrow \Gamma(T Q \oplus V)$ given by $v \mapsto 0 \oplus v$ are Lie algebra homomorphisms.

(ii) Let $\varphi \in C^{\infty}(Q), X_{i} \in \Gamma(T Q)$, and $v_{i} \in \Gamma(V), i=1,2$, be given. Then we have

$$
\begin{aligned}
{\left[v_{1}, \varphi v_{2}\right] } & =\varphi\left[v_{1}, v_{2}\right] \\
{\left[X_{1}, \varphi X_{2}\right] } & =X_{1}[\varphi] X_{2}+\varphi\left[X_{1}, X_{2}\right] \\
{\left[X_{1}, \varphi v_{2}\right] } & =X_{1}[\varphi] v_{2}+\varphi\left[X_{1}, v_{2}\right] \\
{\left[\varphi X_{1}, v_{2}\right] } & =\varphi\left[X_{1}, v_{2}\right] \\
{\left[X_{1} \oplus v_{1}, \varphi\left(X_{2} \oplus v_{2}\right)\right] } & =X_{1}[\varphi]\left(X_{2} \oplus v_{2}\right)+\varphi\left[X_{1} \oplus v_{1}, X_{2} \oplus v_{2}\right] .
\end{aligned}
$$

Let us denote $W:=T Q \oplus V$ and also $w_{i}:=X_{i} \oplus v_{i}$, for $i=1,2$. Define $w_{1}[\varphi]:=$ $X_{1}[\varphi]$. Then the previous equalities can be converted into a single equality

$$
\left[w_{1}, \varphi w_{2}\right]=\varphi\left[w_{1}, w_{2}\right]+w_{1}[\varphi] w_{2}
$$

\section{Morphisms between Lagrange-Poincaré Bundles.}

The nature of morphisms between Lagrange-Poincaré bundles is clarified by the following.

Lemma 6.1.3. (i) Let $T Q \oplus V$ be an object of $\mathfrak{L P}$ and let $\nabla, \omega,[$,$] be the$ structure on $V$. Assume that there is a structure $\nabla^{\prime}, \omega^{\prime},[,]^{\prime}$ on $V$ such that the Lie algebra on $\Gamma(T Q \oplus V)$ defined by $\nabla^{\prime}, \omega^{\prime},[,]^{\prime}$ is the same as the Lie algebra defined by $\nabla, \omega,[$,$] . Then \nabla^{\prime}=\nabla, \omega^{\prime}=\omega$, and $[,]^{\prime}=[$,$] .$ 
(ii) Let $T Q_{i} \oplus V_{i}$ be objects of $\mathfrak{L P}$ for $i=1,2$. Let $f: T Q_{1} \oplus V_{2} \rightarrow T Q_{2} \oplus V_{2}$ be a vector bundle isomorphism. Assume that

$$
f_{*}: \Gamma\left(T Q_{1} \oplus V_{2}\right) \rightarrow \Gamma\left(T Q_{2} \oplus V_{2}\right)
$$

is a Lie algebra homomorphism. Then $f\left(V_{1}\right)=V_{2}$. Assume, in addition, that $f\left(T Q_{1}\right) \subset T Q_{2}$. Then $f$ is an isomorphism in the category $\mathfrak{L P}$, that is, $f$ is an isomorphism of Lagrange-Poincaré bundles.

Proof. (i) By hypothesis, we have, for all $X_{i} \oplus u_{i} \in \Gamma(T Q \oplus V), i=1,2$,

$$
\begin{aligned}
& {\left[X_{1}, X_{2}\right] \oplus \nabla_{X_{1}} u_{2}-\nabla_{X_{2}} u_{1}-\omega\left(X_{1}, X_{2}\right)+\left[u_{1}, u_{2}\right]} \\
& \quad=\left[X_{1}, X_{2}\right] \oplus \nabla_{X_{1}}^{\prime} u_{2}-\nabla_{X_{2}}^{\prime} u_{1}-\omega^{\prime}\left(X_{1}, X_{2}\right)+\left[u_{1}, u_{2}\right]^{\prime} .
\end{aligned}
$$

Taking $u_{1}=u_{2}=0$ and $X_{i}$ arbitrary for $i=1,2$, we obtain $\omega^{\prime}=\omega$. Taking $X_{1}=X_{2}=0$ and $u_{i}$ arbitrary for $i=1,2$, we obtain [, $]^{\prime}=\left[\right.$, ]. Taking $u_{1}=0$, $X_{2}=0$ and $u_{2}, X_{1}$ arbitrary, we obtain $\nabla^{\prime}=\nabla$.

(ii) First, we will prove that $f\left(V_{1}\right)=V_{2}$. By contradiction, assume that there is an element $\bar{v}_{1} \in V_{1}$ such that $f\left(\bar{v}_{1}\right)=\bar{X}_{2} \oplus \bar{v}_{2}$, where $\bar{X}_{2} \in T_{q_{2}} Q_{2}$, satisfies $\bar{X}_{2} \neq 0$. Then there exists $\varphi_{2} \in C^{\infty}\left(Q_{2}\right)$ such that $\mathbf{d} \varphi\left(q_{2}\right)\left(\bar{X}_{2}\right) \neq 0$. Since $f$ is a vector bundle isomorphism, the induced map on the base $f_{0}: Q_{1} \rightarrow Q_{2}$ is a diffeomorphism. Let $q_{1}=f_{0}^{-1}\left(q_{2}\right)$ and $\varphi_{1}=f_{0}^{*} \varphi_{2}=\varphi_{2} \circ f_{0}$. Let $v_{1} \in \Gamma\left(V_{1}\right)$ be such that $v_{1}\left(q_{1}\right)=\bar{v}_{1}$ and let $f_{*} v_{1}=X_{2} \oplus v_{2}$. Thus, $X_{2}\left(q_{2}\right) \oplus v_{2}\left(q_{2}\right)=\bar{X}_{2} \oplus \bar{v}_{2}$. We obviously have $\left[\varphi_{1} v_{1}, v_{1}\right]=\varphi_{1}\left[v_{1}, v_{1}\right]=0$, and therefore $f_{*}\left[\varphi_{1} v_{1}, v_{1}\right]=0$.

On the other hand, using the fact that $f_{*}$ is a Lie algebra isomorphism and also Lemma 6.1.2, we have

$$
\begin{aligned}
f_{*}\left[\varphi_{1} v_{1}, v_{1}\right] & =\left[\varphi_{2}\left(X_{2} \oplus v_{2}\right), X_{2} \oplus v_{2}\right] \\
& =-X_{2}\left[\varphi_{2}\right]\left(X_{2} \oplus v_{2}\right)+\varphi_{2}\left[X_{2} \oplus v_{2}, X_{2} \oplus v_{2}\right] \\
& =-X_{2}\left[\varphi_{2}\right]\left(X_{2} \oplus v_{2}\right),
\end{aligned}
$$

which gives a contradiction since $X_{2}\left[\varphi_{2}\right]\left(q_{2}\right)=\mathbf{d} \varphi\left(q_{2}\right)\left(X_{2}\right) \neq 0$. We have proven hence that $f\left(V_{1}\right) \subset V_{2}$.

Replacing in the above argument $f$ by the vector bundle isomorphism $f^{-1}$ : $T Q_{2} \oplus V_{2} \rightarrow T Q_{1} \oplus V_{1}$ and using the fact that $\left(f^{-1}\right)_{*}$ is a Lie algebra isomorphism between the corresponding spaces of sections, it follows that $f^{-1}\left(V_{2}\right) \subset V_{1}$. Therefore, $f\left(V_{1}\right)=V_{2}$.

Now let $\nabla_{i}, \omega_{i},[,]_{i}$ be the structure on the bundle $V_{i}$ for $i=1,2$, and assume that $f\left(T Q_{1}\right) \subset T Q_{2}$. Since $f$ is a vector bundle isomorphism and $f\left(V_{1}\right)=V_{2}$, it follows that $f\left(T Q_{1}\right)=T Q_{2}$. However $f_{*}$ is a Lie algebra isomorphism so we get for 
any $X \oplus v, X^{\prime} \oplus v^{\prime} \in \Gamma\left(T Q_{1} \oplus V_{1}\right)$,

$$
\begin{aligned}
f_{*}[X, & \left.X^{\prime}\right] \oplus f_{*} \nabla_{1 X} v^{\prime}-f_{*} \nabla_{1 X^{\prime}} v-f_{*} \omega_{1}\left(X, X^{\prime}\right)+f_{*}\left[v, v^{\prime}\right]_{1} \\
& =f_{*}\left(\left[X, X^{\prime}\right] \oplus \nabla_{1 X} v^{\prime}-\nabla_{1 X^{\prime}} v-\omega_{1}\left(X, X^{\prime}\right)+\left[v, v^{\prime}\right]_{1}\right) \\
& =f_{*}\left[X \oplus v, X^{\prime} \oplus v^{\prime}\right]_{1} \\
& =\left[f_{*}(X \oplus v), f_{*}\left(X^{\prime} \oplus v^{\prime}\right)\right]_{2} \\
& =\left[f_{*} X \oplus f_{*} v, f_{*} X^{\prime} \oplus f_{*} v^{\prime}\right]_{2} \\
& =\left[f_{*} X, f_{*} X^{\prime}\right] \oplus \nabla_{2 f_{*} X} f_{*} v^{\prime}-\nabla_{2 f_{*} X^{\prime}} f_{*} v-\omega_{2}\left(f_{*} X, f_{*} X^{\prime}\right)+\left[f_{*} v, f_{*} v^{\prime}\right]_{2} .
\end{aligned}
$$

Taking $X=X^{\prime}=0$, we get $f_{*}\left[v, v^{\prime}\right]_{1}=\left[f_{*} v, f_{*} v^{\prime}\right]_{2}$. Next, taking $X^{\prime}=0$ and $v=0$ we obtain $f_{*} \nabla_{1 X} v^{\prime}=\nabla_{2 f_{*} X} f_{*} v^{\prime}$. Finally, taking $v=v^{\prime}=0$, we have $f_{*} \omega_{1}\left(X, X^{\prime}\right)=\omega_{2}\left(f_{*} X, f_{*} X^{\prime}\right)$ and also $f_{*}\left[X, X^{\prime}\right]=\left[f_{*} X, f_{*} X^{\prime}\right]$.

To show that $f$ is a morphism of Lagrange-Poincaré bundles (i.e., a morphism in the category $\mathfrak{L P})$, it remains to show that $f \mid T Q_{1}=T f_{0}$. Let $X \in \Gamma\left(T Q_{1}\right)=$ $\mathfrak{X}^{\infty}\left(Q_{1}\right)$ and $\varphi \in C^{\infty}\left(Q_{1}\right)$. Then we have

$$
f_{*}[X, \varphi X]=f_{*}(X[\varphi] X)=f_{*}(X[\varphi]) f_{*} X .
$$

On the other hand,

$$
f_{*}[X, \varphi X]=\left[f_{*} X, f_{*}(\varphi X)\right]=\left[f_{*} X, f_{0 *} \varphi f_{*} X\right]=\left(\left(f_{*} X\right)\left[f_{0 *} \varphi\right]\right)\left(f_{*} X\right) .
$$

Therefore we have proven that for any $X \in \Gamma\left(T Q_{1}\right)$ and $\varphi \in C^{\infty}\left(Q_{1}\right)$ we have $f_{*}(X[\varphi])=\left(f_{*} X\right)\left[f_{0 *} \varphi\right]$. Since $f\left(T Q_{1}\right)=T Q_{2}$, this implies $f \mid T Q_{1}=T f_{0}$ as we shall show below.

Let $q_{2} \in Q_{2}$ and denote $q_{1}=f_{0}^{-1}\left(q_{2}\right)$. Then using the above relation in the third equality below, we get

$$
\begin{aligned}
\mathbf{d}\left(\varphi \circ f_{0}^{-1}\right)\left(q_{2}\right) \cdot T_{q_{1}} f_{0}\left(X\left(q_{1}\right)\right) & =\mathbf{d} \varphi\left(q_{1}\right) \cdot X\left(q_{1}\right)=\left(X[\varphi] \circ f^{-1}\right)\left(q_{2}\right) \\
& =\left(f_{*} X\right)\left[f_{0 *} \varphi\right]\left(q_{2}\right)=\mathbf{d}\left(\varphi \circ f_{0}^{-1}\right)\left(q_{2}\right) \cdot\left(f_{*} X\right)\left(q_{2}\right) \\
& =\mathbf{d}\left(\varphi \circ f_{0}^{-1}\right)\left(q_{2}\right) \cdot f\left(X\left(q_{1}\right)\right),
\end{aligned}
$$

that is, $f\left(X\left(q_{1}\right)\right)=T_{q_{1}} f_{0}\left(X\left(q_{1}\right)\right)$ for any $X_{1} \in \mathfrak{X}^{\infty}\left(Q_{1}\right)$ which proves that $f \mid T Q_{1}=$ $T f_{0}$.

\section{Morphism-related Sections of Lagrange-Poincaré Bundles.}

Let $f: T Q_{1} \oplus V_{1} \rightarrow T Q_{2} \oplus V_{2}$ be a given morphism of Lagrange-Poincaré bundles and let $X \oplus u \in \Gamma\left(T Q_{1} \oplus V_{1}\right)$ and $Y \oplus v \in \Gamma\left(T Q_{2} \oplus V_{2}\right)$ be given. Then $X \oplus u$ and $Y \oplus v$ are said to be $f$-related if

$$
f(X(q) \oplus u(q))=Y\left(f_{0}(q)\right) \oplus v\left(f_{0}(q)\right)
$$

for all $q \in Q_{1}$. In particular, it is easy to prove that if $X \oplus u$ and $Y \oplus v$ are $f$-related then $X$ and $Y$ are $f_{0}$-related as vector fields according to the usual definition. 
Lemma 6.1.4. Let $f: T Q_{1} \oplus V_{1} \rightarrow T Q_{2} \oplus V_{2}$ be a morphism of Lagrange-Poincaré bundles and let $X_{i} \oplus u_{i} \in \Gamma\left(T Q_{1} \oplus V_{1}\right), Y_{i} \oplus v_{i} \in \Gamma\left(T Q_{2} \oplus V_{2}\right)$ for $i=1,2$, be given. Assume that $X_{i} \oplus u_{i}$ and $Y_{i} \oplus v_{i}$ are $f$-related for $i=1,2$. Then $\left[X_{1} \oplus u_{1}, X_{2} \oplus u_{2}\right]$ and $\left[Y_{1} \oplus v_{1}, Y_{2} \oplus v_{2}\right]$ are also $f$-related.

Proof. Since $X_{i} \oplus u_{i}$ and $Y_{i} \oplus v_{i}$ are $f$-related for $i=1,2$, Definition 6.1.1 implies for all $q \in Q_{1}$

$$
\begin{aligned}
f\left(\left[X_{1} \oplus u_{1}, X_{2} \oplus u_{2}\right](q)\right) \\
\quad=f\left(\left(\left[X_{1}, X_{2}\right] \oplus \nabla_{X 1} u_{2}-\nabla_{X 2} u_{1}-\omega\left(X_{1}, X_{2}\right)+\left[u_{1}, u_{2}\right]\right)(q)\right) \\
\quad=\left[Y_{1}, Y_{2}\right]\left(f_{0}(q)\right) \oplus \nabla_{Y_{1}} v_{2}-\nabla_{Y_{2}} v_{1}-\omega\left(Y_{1}, Y_{2}\right)+\left[v_{1}, v_{2}\right]\left(f_{0}(q)\right) \\
\quad=\left[Y_{1} \oplus v_{1}, Y_{2} \oplus v_{2}\right]\left(f_{0}(q)\right) .
\end{aligned}
$$

Lemma 6.1.5. Let $f: T Q_{1} \oplus V_{1} \rightarrow T Q_{2} \oplus V_{2}$ be a morphism in $\mathfrak{L P}$ and assume, in addition, that $f$ is a linear isomorphism on each fiber. Then $f^{*}: \Gamma\left(T Q_{2} \oplus V_{2}\right) \rightarrow$ $\Gamma\left(T Q_{1} \oplus V_{1}\right)$ is a Lie algebra homomorphism.

Proof. Using the remarks preceding Lemma 5.2.2 it follows that $f^{*}$ is well defined, that for given $Y_{i} \oplus v_{i} \in \Gamma\left(T Q_{2} \oplus V_{2}\right), i=1,2, f^{*}\left(Y_{i} \oplus v_{i}\right) \in \Gamma\left(T Q_{1} \oplus V_{1}\right)$, and that $f^{*}\left(Y_{i} \oplus v_{i}\right)=f^{*} Y_{i} \oplus f^{*} v_{i}$ and $Y_{i} \oplus v_{i}$ are $f$-related for $i=1,2$. Then, by Lemma 6.1.4, $\left[f^{*}\left(Y_{1} \oplus v_{1}\right), f^{*}\left(Y_{2} \oplus v_{2}\right)\right]$ and $\left[Y_{1} \oplus v_{1}, Y_{2} \oplus v_{2}\right]$ are also $f$-related. However, the hypothesis of the lemma implies that there is one and only one section of $T Q_{1} \oplus V_{1}$ which is $f$-related to a given section of $T Q_{2} \oplus V_{2}$, so we can conclude

$$
\left[f^{*}\left(Y_{1} \oplus v_{1}\right), f^{*}\left(Y_{2} \oplus v_{2}\right)\right]=f^{*}\left(\left[Y_{1} \oplus v_{1}, Y_{2} \oplus v_{2}\right]\right) .
$$

\section{Group Actions on Vector Bundles with a Structure [,], $\omega, \nabla$.}

As we have already indicated, when tangent bundles are reduced, one enters the category of Lagrange-Poincaré bundles and so, to continue the process of reduction, it is important to know how these objects themselves behave under reduction. The first job is to examine group actions on vector bundles with the extra structure given in Definition 6.1.1.

Let $\tau^{i}: V_{i} \rightarrow Q_{i}, i=1,2$, be vector bundles with extra structure $[,]_{i}, \omega_{i}$, $\nabla_{i}$ satisfying conditions (a), (b), (c) of Definition 6.1.1A. A vector bundle map $f: V_{1} \rightarrow V_{2}$ is called a morphism if it commutes with the structures given by $[,]_{i}$, $\omega_{i}, \nabla_{i}, i=1,2$, that is, if the conditions of Definition 6.1.1B(b) hold.

Definition 6.1.6. An action $\rho: G \times V \rightarrow V$ of a Lie group $G$ on a vector bundle $\tau: V \rightarrow Q$ with extra structure [,], $\omega, \nabla$ as in Definition $6.1 .1 \mathrm{~A}$, is a vector bundle action such that, for each $g \in G, \rho_{g}: V \rightarrow V$ is a morphism in the previous sense. More precisely, we assume 
(a) $g\left[v_{1}, v_{2}\right]=\left[g v_{1}, g v_{2}\right]$ for all $g \in G$ and all $v_{1}, v_{2} \in V$ satisfying $\tau\left(v_{1}\right)=\tau\left(v_{2}\right)$;

(b) $g \omega(X, Y)=\omega(g X, g Y)$, for all $g \in G$ and all $X, Y \in T Q$ satisfying $\tau_{Q}(X)=$ $\tau_{Q}(Y)$;

(c)

$$
\frac{D g v(t)}{D t}=g \frac{D v(t)}{D t}
$$

for any curve $v(t)$ on $V$ and any $g \in G$.

Now we shall state the following lemma whose proof is straightforward.

Lemma 6.1.7. Let $\tau^{i}: V_{i} \rightarrow Q_{i}, i=1,2$, be vector bundles with an extra structure given only by [,], $\omega$ satisfying conditions (a), (b) of Definition 6.1.1A. Let $\rho: G \times V \rightarrow V$ be a vector bundle action. Assume that [,] or $\omega$ are invariant relative to the action $\rho$, that is, condition (a) or (b) of Definition 6.1.6 holds. Then there are corresponding structures $[,]_{G},[\omega]_{G}$ on the quotient vector bundle $V / G$. These quotient structures are naturally defined by:

(a) $\left[\left[v_{1}\right]_{G},\left[v_{2}\right]_{G}\right]=\left[\left[v_{1}, v_{2}\right]\right]_{G}$ for all $v_{1}, v_{2} \in V$ such that $\tau\left(v_{1}\right)=\tau\left(v_{2}\right)$, and

(b) $[\omega]_{G}\left([X]_{G},[Y]_{G}\right)=[\omega(X, Y)]_{G}$ for all $X, Y \in T Q$ such that $\tau_{Q}(X)=\tau_{Q}(Y)$.

We will sometimes use the isomorphism $\alpha_{A}$ of Lemma 2.4.2 as an identification and, consequently, write $[X]_{G} \equiv T \pi(X) \oplus \bar{\xi},[Y]_{G} \equiv T \pi(Y) \oplus \bar{\eta}$. Thus, the second identity in the above lemma becomes

$$
\begin{aligned}
{[\omega]_{G} } & \left([X]_{G},[Y]_{G}\right) \\
& \equiv[\omega]_{G}(T \pi(X) \oplus \bar{\xi}, T \pi(Y) \oplus \bar{\eta}) \\
& =[\omega]_{G}(T \pi(X), T \pi(Y))+[\omega]_{G}(T \pi(X), \bar{\eta})+[\omega]_{G}(\bar{\xi}, T \pi(Y))+[\omega]_{G}(\bar{\xi}, \bar{\eta}) .
\end{aligned}
$$

\section{Invariance Properties of Covariant Derivatives.}

To pass covariant derivatives to quotient bundles, we will need some preparatory lemmas.

Lemma 6.1.8. Let $\tau: V \rightarrow Q$ be a vector bundle and let $D / D t$ be a covariant derivative along curves associated to an affine connection $\nabla$ on $V$ (see the paragraphs following Definition 2.3.1 and Lemma 2.3.2). Let $\rho: G \times V \rightarrow V$ be a vector bundle action. If $\rho$ commutes with $D$ in the sense of Definition 6.1.6(c), then $\nabla$ is invariant in the following sense:

$$
\nabla_{g_{*}} g_{*} v=g_{*} \nabla_{X} v
$$

for all $X \in \mathfrak{X}^{\infty}(Q)$, all $v \in \Gamma(V)$, and all $g \in G$. Conversely, if $\nabla$ is invariant then $D / D t$ is invariant.

The proof of this lemma is straightforward. See also the proof of Lemma 6.1.11(b) for a similar argument. 


\section{Horizontal and Vertical Components of Connections.}

To define the notion of quotient covariant derivative or quotient connection, we will need some extra structure, namely, a principal connection on $Q$. Our first job is to synthesize this connection with a given connection on a vector bundle to obtain horizontal covariant derivatives and connections.

Definition 6.1.9. Let $\tau: V \rightarrow Q$ be a vector bundle and let $D / D t$ be the covariant derivative along curves associated to a connection $\nabla$ on $V$. Let $\rho: G \times V \rightarrow V$ be a vector bundle action covering the action $\rho_{0}: G \times Q \rightarrow Q$. Let $A$ be a principal connection on the principal $G$-bundle $Q \rightarrow Q / G$ (relative to the action $\left.\rho_{0}\right)$. Let $v(t)$ be any curve in $V$ and let $q(t)=\tau(v(t))$ for all $t$. Choose $t_{0}$ and let $q_{0}=q\left(t_{0}\right)$. Let $g_{q}(t)$ and $q_{h}(t)$ be as in $\S 2.2$; that is, $q_{h}(t)$ is a horizontal curve, $q(t)=g_{q}(t) q_{h}(t)$, and $g_{q}\left(t_{0}\right)=e$. Then we define

$$
\begin{gathered}
v_{h}(t)=g_{q}^{-1}(t) v(t), \\
\left.\frac{D^{(A, H)} v(t)}{D t}\right|_{t=t_{0}}=\left.\frac{D v_{h}(t)}{D t}\right|_{t=t_{0}},
\end{gathered}
$$

and

$$
\left.\frac{D^{(A, V)} v(t)}{D t}\right|_{t=t_{0}}=\left.\frac{D v(t)}{D t}\right|_{t=t_{0}}-\left.\frac{D^{(A, H)} v(t)}{D t}\right|_{t=t_{0}}
$$

We will call

$$
\frac{D^{(A, H)} v(t)}{D t}
$$

the A-horizontal covariant derivative of $v(t)$ and

$$
\frac{D^{(A, V)} v(t)}{D t}
$$

the A-vertical covariant derivative of $v(t)$. For $X \in \mathfrak{X}^{\infty}(Q)$ and $v \in \Gamma(V)$ we also define $\nabla_{X}^{(A, H)} v$ and $\nabla_{X}^{(A, V)} v$ by

$$
\nabla_{X}^{(A, H)} v\left(q_{0}\right)=\left.\frac{D^{(A, H)}}{D t} v(t)\right|_{t=t_{0}}
$$

and

$$
\nabla_{X}^{(A, V)} v\left(q_{0}\right)=\left.\frac{D^{(A, V)}}{D t} v(t)\right|_{t=t_{0}},
$$

where the covariant derivatives on the right hand side are taken along any smooth curve $q(t)$ in $Q$ satisfying $q\left(t_{0}\right)=q_{0}, \dot{q}\left(t_{0}\right)=X\left(q_{0}\right)$, and $v(t)=v(q(t)$ ) for all t. We will call $\nabla^{(A, H)}$ the A-horizontal component and $\nabla^{(A, V)}$ the A-vertical component of the connection $\nabla$. 
We see from this definition that

$$
\nabla_{X} v\left(q_{0}\right)=\nabla_{X}^{(A, H)} v\left(q_{0}\right)+\nabla_{X}^{(A, V)} v\left(q_{0}\right)
$$

The following lemma gives, in particular, an alternative characterization of $\nabla_{X}^{(A, H)} v$ and $\nabla_{X}^{(A, V)} v$ for a $G$-invariant section $v$ of $V$. It also shows that, when restricted to invariant sections $v \in \Gamma^{G}(V)$, the operator $\nabla^{(A, H)}$ has the formal properties of a connection.

Lemma 6.1.10. (a) Let $\operatorname{Hor}^{A}(X) \equiv X_{H}$ and $\operatorname{Ver}^{A}(X) \equiv X_{V}$, for short. Then we have, for each $q_{0} \in Q$, each $X \in \mathfrak{X}^{\infty}(Q)$, and each $v \in \Gamma^{G}(V)$,

$$
\nabla_{X}^{(A, H)} v\left(q_{0}\right)=\nabla_{X_{H}} v\left(q_{0}\right)
$$

and

$$
\nabla_{X}^{(A, V)} v\left(q_{0}\right)=\nabla_{X_{V}} v\left(q_{0}\right)
$$

In particular,

$$
\nabla_{X} v\left(q_{0}\right)=\nabla_{X_{H}} v\left(q_{0}\right)+\nabla_{X_{V}} v\left(q_{0}\right)
$$

(b) Let $v \in \Gamma^{G}(V)$ and let $q(t)$ be any curve in $Q$ such that $\dot{q}\left(t_{0}\right)=X\left(q_{0}\right)$. Define $q_{h}(t)$ and $g_{q}(t)$ as in Definition 6.1 .9 (see also §2.1). Then

$$
\nabla_{X}^{(A, H)} v\left(q_{0}\right)=\left.\frac{D}{D t} g_{q}^{-1}(t) v(t)\right|_{t=t_{0}}=\left.\frac{D}{D t} v\left(q_{h}(t)\right)\right|_{t=t_{0}}
$$

and

$$
\nabla_{X}^{(A, V)} v\left(q_{0}\right)=\left.\frac{D}{D t} g_{q}(t) v\left(t_{0}\right)\right|_{t=t_{0}} .
$$

In particular, $\nabla_{X}^{(A, V)} v\left(q_{0}\right)$ depends only on $\xi_{0}=\dot{g}_{q}\left(t_{0}\right)$ and $v\left(q_{0}\right)=v\left(t_{0}\right)$.

(c) Let $v \in \Gamma^{G}(V)$, let $q(t)$ be any curve in $Q$, and let $v(t)=v(q(t))$ for all $t$. Then

$$
\left.\frac{D^{(A, H)}}{D t} v(t)\right|_{t=t_{0}}=\left.\frac{D}{D t} g_{q}^{-1}(t) v(t)\right|_{t=t_{0}}=\left.\frac{D}{D t} v\left(q_{h}(t)\right)\right|_{t=t_{0}}
$$

and

$$
\left.\frac{D^{(A, V)}}{D t} v(t)\right|_{t=t_{0}}=\left.\frac{D}{D t} g_{q}(t) v\left(t_{0}\right)\right|_{t=t_{0}} .
$$

Proof. (a) Let $q(t)$ be any curve in $Q$ such that $\dot{q}\left(t_{0}\right)=X\left(q_{0}\right)$ and let $v(t)=$ $v(q(t))$ for all $t$. Then we can easily see that $\tau\left(v_{h}(t)\right)=q_{h}(t)$ for all $t$. Since $v$ is $G$-invariant we have $v(t)=g_{q}(t) v\left(q_{h}(t)\right)$. This argument and the definition of $\nabla$, $\nabla^{(A, H)}$, and $\nabla^{(A, V)}$ imply the formulas in part (a) of the lemma.

(b) This part is basically a restatement of part (a). The first equality is a direct consequence of the definition of $\nabla^{(A, H)}$. Now Let $\xi_{0}=\dot{g}_{q}\left(t_{0}\right)$. Then

$$
X_{V}\left(q_{0}\right)=\xi_{0} q_{0}=\left.\frac{d}{d t}\left(g_{q}(t) q_{0}\right)\right|_{t=t_{0}}
$$


Therefore the second equality is a consequence of the second equality of part (a) and the $G$-invariance of $v$.

(c) To prove this part we can proceed essentially as in the proof of parts (a) and (b). Alternatively, we can deduce it directly from part (b).

As a technical point, we note that in the proof of the previous lemma we are strongly using the fact that the covariant derivative $D / D t$ is defined by a connection $\nabla$ which, in particular, has the formal property $\nabla_{X+Y}=\nabla_{X}+\nabla_{Y}$.

The next two lemmas establish some basic properties of invariant covariant derivatives and invariant connections.

Lemma 6.1.11. (a) Assume the same hypothesis as in Definition 6.1 .9 and, in addition, that $D / D t$ is $G$-invariant in the sense of Definition 6.1.6(c). Then

$$
\frac{D^{(A, H)}}{D t} \text { and } \frac{D^{(A, V)}}{D t}
$$

are also $G$-invariant in the following sense:

$$
\frac{D^{(A, H)} g v(t)}{D t}=g \frac{D^{(A, H)} v(t)}{D t}
$$

and

$$
\frac{D^{(A, V)} g v(t)}{D t}=g \frac{D^{(A, V)} v(t)}{D t}
$$

for all curves $v(t)$ in $V$ and all $g \in G$.

(b) Let $\nabla^{(A, H)}$ and $\nabla^{(A, V)}$ be the A-horizontal and A-vertical components of a given connection $\nabla$ on $V$ and assume that $\nabla$ is $G$-invariant. Then $\nabla^{(A, H)}$ and $\nabla^{(A, V)}$ are also $G$-invariant in the following sense:

$$
\nabla_{g_{*} X}^{(A, H)} g_{*} v=g_{*} \nabla_{X}^{(A, H)} v
$$

and

$$
\nabla_{g_{*} X}^{(A, V)} g_{*} v=g_{*} \nabla_{X}^{(A, V)} v,
$$

for all $X \in \mathfrak{X}^{\infty}(Q)$, all $v \in \Gamma(V)$, and all $g \in G$.

Proof. (a) Let $a \in G$ and $v(t)$ be given. Let $\tau(v(t))=q(t)$. Then we immediately have $\tau(a v(t))=a q(t)$. For any chosen $t_{0}$ we have $a q(t)=g_{a q}(t)(a q)_{h}(t)$ and $q(t)=$ $g_{q}(t) q_{h}(t)$ with $g_{a q}\left(t_{0}\right)=g_{q}\left(t_{0}\right)=e$. We know that $(a q)_{h}(t)=a q_{h}(t)$ for all $t$. Using these equalities we can easily see that $g_{a q}(t)=a g_{q}(t) a^{-1}$ for all $t$. Then

$$
\begin{aligned}
\left.\frac{D^{(A, H)}}{D t} a v(t)\right|_{t=t_{0}} & =\left.\frac{D}{D t} g_{a q}^{-1}(t) a v(t)\right|_{t=t_{0}}=\left.\frac{D}{D t} a g_{q}^{-1}(t) a^{-1} a v(t)\right|_{t=t_{0}} \\
& =\left.\frac{D}{D t} a g_{q}^{-1}(t) v(t)\right|_{t=t_{0}}=\left.\frac{D}{D t} a v_{h}(t)\right|_{t=t_{0}} \\
& =\left.a \frac{D}{D t} v_{h}(t)\right|_{t=t_{0}}=\left.a \frac{D^{(A, H)}}{D t} v(t)\right|_{t=t_{0}}
\end{aligned}
$$


To prove that

$$
\frac{D^{(A, V)} a v(t)}{D t}=a \frac{D^{(A, V)} v(t)}{D t}
$$

for all curves $v(t)$ on $V$ and all $a \in G$ we simply use Definition 6.1.9 and invariance of $D / D t$ and of $D^{(A, H)} / D t$.

(b) To prove invariance of $\nabla^{(A, H)}$ and $\nabla^{(A, V)}$ we use part (a) and the definitions of $\nabla^{(A, H)}$ and $\nabla^{(A, V)}$.

To prove part (b) of the preceding lemma for invariant $v$, we can use, alternatively, Lemma 6.1.10 and recall that for any $g \in G$ and any $X \in \mathfrak{X}^{\infty}(Q)$ we have $\left(g_{*} X\right)_{H}=$ $g_{*} X_{H}$ and also $\left(g_{*} X\right)_{V}=g_{*} X_{V}$.

Lemma 6.1.12. Assume the same hypothesis as in Lemma 6.1.11. Then for any curve $a(t)$ on $G$ and any curve $v(t)$ in $V$ we have

$$
\frac{D^{(A, H)}}{D t}[a(t) v(t)]=a(t) \frac{D^{(A, H)}}{D t} v(t) .
$$

Proof. If $q(t)=\tau(v(t))$, then $a(t) q(t)=\tau(a(t) v(t))$. Choose $t_{0}$. Then we have, for all $t, g_{a q}(t)(a q)_{h}(t)=a(t) q(t)$ and also $g_{q}(t) q_{h}(t)=q(t)$, where $g_{a q}\left(t_{0}\right)=$ $g_{q}\left(t_{0}\right)=e$. We have $(a q)_{h}(t)=a\left(t_{0}\right) q_{h}(t)$ for all $t$, since both curves are horizontal, both pass through the same point $a\left(t_{0}\right) q\left(t_{0}\right)$ at $t=t_{0}$, and both project to the same curve in $Q / G$. Therefore $a(t) q(t)=g_{a q}(t) a\left(t_{0}\right) q_{h}(t)$, for all $t$. On the other hand, $a(t) q(t)=a(t) g_{q}(t) q_{h}(t)$ which then implies that $g_{a q}(t)=a(t) g_{q}(t)\left(a\left(t_{0}\right)\right)^{-1}$. Using this and the $G$-invariance of $D / D t$ we obtain

$$
\begin{aligned}
\left.\frac{D^{(A, H)}}{D t} a(t) v(t)\right|_{t=t_{0}} & =\left.\frac{D}{D t} a\left(t_{0}\right)\left(g_{q}(t)\right)^{-1} a^{-1}(t) a(t) v(t)\right|_{t=t_{0}} \\
& =\left.a\left(t_{0}\right) \frac{D}{D t}\left(g_{q}(t)\right)^{-1} v(t)\right|_{t=t_{0}} \\
& =\left.a\left(t_{0}\right) \frac{D^{(A, H)}}{D t} v(t)\right|_{t=t_{0}} .
\end{aligned}
$$

\section{Two More Properties of Covariant Derivatives.}

The next two lemmas are included for the sake of completeness only, although they will not be used in an essential way in the present work.

Lemma 6.1.13. Assume the same conditions as in Definition 6.1.9. Let $v(t)$ be a curve in $V, q(t)=\tau(v(t))$, and let $f(t)$ be a real valued function. Then

$$
\frac{D^{(A, H)}}{D t}[f(t) v(t)]=\dot{f}(t) v(t)+f(t) \frac{D^{(A, H)}}{D t} v(t)
$$


for all $t$ and

$$
\frac{D^{(A, V)}}{D t}[f(t) v(t)]=f(t) \frac{D^{(A, V)}}{D t} v(t)
$$

for all $t$.

Proof. It is not difficult to prove this lemma from the definitions. We will omit the details.

The following lemma generalizes Lemma 6.1.12

Lemma 6.1.14. For each curve $q(t)$ in $Q$ and each choice of $t_{0}$ define $\xi\left(t_{0}\right)=$ $\dot{g}_{q}\left(t_{0}\right)$. Thus, as $t_{0}$ varies, $\xi\left(t_{0}\right)$ describes a curve in $\mathfrak{g}$ which we will denote $\xi_{q}(t)$. Assume the same conditions as in Definition 6.1.9. Now let $v(t)$ be a curve in $V$ and let $q(t)=\tau(v(t))$ for all $t$. Let $a(t)$ be any curve in $G$. Then we have

(a)

$$
\left.\frac{D^{(A, H)}}{D t}[a(t) v(t)]\right|_{t=t_{0}}=\left.\frac{D}{D t}\left[a\left(t_{0}\right)\left(g_{q}(t)\right)^{-1} v(t)\right]\right|_{t=t_{0}}
$$

(b)

$$
\xi_{a q}(t)=\operatorname{Ad}_{a(t)} \xi_{q}(t)+\dot{a}(t) a^{-1}(t)
$$

for all t. For each $t$,

$$
\frac{D^{(A, V)}}{D t}[a(t) v(t)]
$$

only depends on $a(t) v(t)$ and $\xi_{a q}(t)$.

Proof. (a) The proof of this part is an immediate consequence of the last part of the proof of Lemma 6.1.12.

(b) From the proof of Lemma 6.1 .12 it follows that $g_{a q}(t)=a(t) g_{q}(t)\left(a\left(t_{0}\right)\right)^{-1}$. Using this, we can easily prove that $\xi_{a q}(t)=\operatorname{Ad}_{a(t)} \xi_{q}(t)+\dot{a}(t) a^{-1}(t)$.

\subsection{Reduction of Lagrange-Poincaré Bundles}

Now we embark on the task of reducing Lagrange-Poincaré bundles. The goal is to see how the geometric structures on these bundles pass to the quotient bundle so that the result is still a Lagrange-Poincaré bundle. Most of this structure passes to the quotient in a fairly straightforward way. However, things are not as simple with the covariant derivative, so we will need to pay special attention to this.

Now we are ready to pass covariant derivatives as well as horizontal and vertical covariant derivatives and connections to the quotient. 
The notion of quotient connection may be defined without any reference to quotient covariant derivatives. However, we shall treat these two notions together, which will be helpful in our later applications to variational principles.

There are some interesting links between the constructions here and those that appear in the theory of geometric phases (see Marsden, Montgomery and Ratiu (1990), §13), but this will not be pursued in this paper.

\section{Quotient Horizontal Covariant Derivatives and Connections.}

Definition 6.2.1. Assume that the conditions of Definition 6.1 .9 hold and, in addition, assume that $D / D t$ is $G$-invariant; thus the associated connection $\nabla$ on the vector bundle $V$ is also $G$-invariant.

(a) We define the quotient, or reduced, horizontal covariant derivative on the vector bundle $V / G$ by

$$
\left[\frac{D^{(A, H)}}{D t}\right]_{G}[v(t)]_{G}=\left[\frac{D^{(A, H)}}{D t} v(t)\right]_{G}
$$

for any curve $v(t)$ on $V$. (This expression is well defined in view of Lemma 6.1.11a.)

(b) We define the quotient, or reduced, horizontal connection by

$$
\left(\left[\nabla^{(A, H)}\right]_{G}\right)_{[X]_{G}}[v]_{G} \equiv\left[\nabla^{(A, H)}\right]_{G,[X]_{G}}[v]_{G}=\left[\nabla_{X}^{(A, H)} v\right]_{G}
$$

for given $[X]_{G} \in \Gamma(T Q / G)$ and $[v]_{G} \in \Gamma(V / G)$ and corresponding $X \in$ $\Gamma^{G}(T Q)$ and $v \in \Gamma^{G}(V)$. (This expression is well defined in view of Lemma 6.1.11b.)

In this definition, recall that we identify elements of $\Gamma^{G}(T Q)$ with elements of $\Gamma(T Q / G)$ and also elements of $\Gamma^{G}(V)$ with elements of $\Gamma(V / G)$ using Lemma 5.2.2.

The connection associated to $\left[D^{(A, H)} / D t\right]_{G}$ is precisely $\left[\nabla^{(A, H)}\right]_{G}$ in view of Lemmas 6.1.12 and 6.1.11(b).

The reduced horizontal connection can be naturally interpreted as a connection, which we shall denote $\bar{\nabla}$, on the vector bundle $V / G$ in the usual sense. Using the general considerations in the paragraph Affine Connections in $\S 2.3$, this is explained as follows. Let $Y$ be any vector field on $Q / G$ and let $Y^{h}$ be its horizontal lift to $Q$. For any given $[v]_{G} \in \Gamma(V / G)$ define

$$
\bar{\nabla}_{Y}[v]_{G}:=\left[\nabla_{Y^{h}}^{(A, H)} v\right]_{G} .
$$

Then one can check that the properties defining a connection hold for $\bar{\nabla}$. Moreover, we can check that if $X$ is any vector field such that $\operatorname{Hor}^{A}(X)=Y^{h}$, then

$$
\bar{\nabla}_{Y}[v]_{G}=\left[\nabla_{X}^{(A, H)} v\right]_{G} .
$$




\section{Quotient Vertical Covariant Derivatives and Connections.}

It is important for us to define a quotient vertical covariant derivative as well as a quotient vertical connection. However, these will not be a covariant derivative and a connection in the usual sense.

Let us begin with a simple case, namely, the case of a constant curve in $V / G$. This is instructive because the answer turns out to be nonzero.

Let $\left[v_{0}\right]_{G} \in V / G$ be given. Choose $v_{0} \in V$ and $q_{0} \in Q$ such that $\tau\left(v_{0}\right)=q_{0}$. Let $x_{0} \in Q / G$ be given by $x_{0}=(\tau / G)\left(\left[v_{0}\right]_{G}\right)=\pi\left(q_{0}\right)$, where, as before, $\pi: Q \rightarrow Q / G$ is the projection. Let $\xi_{0} \in \mathfrak{g}$ and consider the class $\left[q_{0}, \xi_{0}\right]_{G} \in \tilde{\mathfrak{g}}_{x_{0}}$.

Consider $\left[v_{0}\right]_{G}$ as a constant curve; we define the notion of the vertical covariant derivative of this curve with respect to $\left[q_{0}, \xi_{0}\right]_{G}$, as follows. Choose a curve $g_{0}(t)$ in $G$ such that $g_{0}\left(t_{0}\right)=e$ and $\dot{g}_{0}\left(t_{0}\right)=\xi_{0}$. Let $v_{0}(t)=g_{0}(t) v_{0}$. Define the vertical covariant derivative by

$$
\left.\left[\frac{D^{(A, V)}}{D t}\right]_{G,\left[q_{0}, \xi_{0}\right]_{G}}\left[v_{0}\right]_{G}\right|_{t=t_{0}}=\left[\left.\frac{D^{(A, V)} v_{0}(t)}{D t}\right|_{t=t_{0}}\right]_{G}
$$

Now we have to show that this expression is well defined. First of all, we need to explain why the left hand side has the new subscript $\left[q_{0}, \xi_{0}\right]_{G}$. This notation means that the class on the right hand side depends on the class $\left[q_{0}, \xi_{0}\right]_{G}$. The reason for this is as follows: during the quotient process, one represents the class $\left[v_{0}\right]_{G}$ by an element of $V$, say $g(t) v_{0}$; the point is that the representative depends on a group element and this group element could be time dependent without changing the class $\left[v_{0}\right]_{G}$. The class of the resulting covariant derivative then depends on the class of $\dot{g}$, so this dependence on $\left[q_{0}, \xi_{0}\right]_{G}$ cannot be eliminated.

We now examine this in more detail, still using the constant curve $\left[v_{0}\right]_{G}$ in the quotient. First of all, note that from Definition 6.1.9, and the fact that the quotient curve $x_{0}$ in $Q / G$ is constant,

$$
\left.\frac{D^{(A, H)} v_{0}(t)}{D t}\right|_{t=t_{0}}=0
$$

and therefore,

$$
\left.\frac{D^{(A, V)} v_{0}(t)}{D t}\right|_{t=t_{0}}=\left.\frac{D v_{0}(t)}{D t}\right|_{t=t_{0}} .
$$

It is clear that the right-hand side does not depend on the choice of the curve $g_{0}(t)$ satisfying $g_{0}\left(t_{0}\right)=e, \dot{g}_{0}\left(t_{0}\right)=\xi_{0}$, and now we shall see that it depends only on $\left[v_{0}\right]_{G} \in V / G$ and $\left[q_{0}, \xi_{0}\right]_{G} \in \tilde{\mathfrak{g}}_{x_{0}}$ and not on the particular choice of $v_{0}$ and $q_{0}$. Let us choose $v_{1} \in\left[v_{0}\right]_{G}$ and also $q_{1} \in\left[q_{0}\right]_{G}$ such that $\tau\left(v_{1}\right)=q_{1}$. There is an $h \in G$ such that $q_{1}=h q_{0}$. Let $\xi_{1}=\operatorname{Ad}_{h} \xi_{0}$, and note that $\left[q_{0}, \xi_{0}\right]_{G}=\left[q_{1}, \xi_{1}\right]_{G}$. Using Lemma 5.2.1, we get $v_{1}=h v_{0}$. If a curve $g_{0}(t)$ on $G$ satisfies $g_{0}\left(t_{0}\right)=e$ and $\dot{g}_{0}\left(t_{0}\right)=\xi_{0}$ then the curve $g_{1}(t)=h g_{0}(t) h^{-1}$ satisfies $g_{1}\left(t_{0}\right)=e$ and $\dot{g}_{1}\left(t_{0}\right)=\xi_{1}$. Define $v_{1}(t)=g_{1}(t) v_{1}$. Then, $v_{1}(t)=h g_{0}(t) h^{-1} h v_{0}=h v_{0}(t)$. Then using Lemma 
6.1.11, we obtain

$$
\left[\left.\frac{D^{(A, V)} v_{1}(t)}{D t}\right|_{t=t_{0}}\right]_{G}=\left[\left.h \frac{D^{(A, V)} v_{0}(t)}{D t}\right|_{t=t_{0}}\right]_{G}=\left[\left.\frac{D^{(A, V)} v_{0}(t)}{D t}\right|_{t=t_{0}}\right]_{G} .
$$

Thus, we can consistently define the quotient vertical covariant derivative of the constant curve $\left[v_{0}\right]_{G}$ on $V / G$ with respect to $\left[q_{0}, \xi_{0}\right]_{G}$ by equation (6.2.1).

Next we consider the case of general curves. Let $[v]_{G}(t)$ be a curve in $V / G$ and $\left.x(t)=(\tau / G)\left([v]_{G}(t)\right]_{G}\right)$. Choose $\left[q_{0}, \xi_{0}\right]_{G} \in \tilde{\mathfrak{g}}_{x_{0}}$, where $x_{0}=x\left(t_{0}\right)=(\tau / G)\left([v]_{G}\left(t_{0}\right)\right)$. We want to define the covariant derivative of $[v]_{G}(t)$ with respect to $\left[q_{0}, \xi_{0}\right]_{G}$ at $t=t_{0}$. Choose $v_{0} \in V$ and $q_{0} \in Q$ such that $\tau\left(v_{0}\right)=q_{0}$. Choose a curve $g_{0}(t)$ on $G$ such that $g_{0}\left(t_{0}\right)=e$ and $\dot{g}_{0}\left(t_{0}\right)=\xi_{0}$. Lemma 5.2.1 shows that there is a unique curve $v_{q_{0}}^{h}(t)$ in $V$ such that $\left[v_{q_{0}}^{h}(t)\right]_{G}=[v]_{G}(t)$ and $\tau\left(v_{q_{0}}^{h}(t)\right)=x_{q_{0}}^{h}(t)$ for all $t$. Let $v_{0}(t)=g_{0}(t) v_{q_{0}}^{h}(t)$ and $q_{0}(t)=g_{0}(t) x_{q_{0}}^{h}(t)$. Again by Lemma 5.2.1, one sees that $v_{0}(t)$ is the only curve in $V$ such that $\left[v_{0}(t)\right]_{G}=[v]_{G}(t)$ and $\tau\left(v_{0}(t)\right)=q_{0}(t)$ for all $t$. Then it is easy to see that

$$
\left.\frac{D^{(A, H)} v_{0}(t)}{D t}\right|_{t=t_{0}}=\left.\frac{D^{(A, H)} v_{0}^{h}(t)}{D t}\right|_{t=t_{0}}=\left.\frac{D v_{0}^{h}(t)}{D t}\right|_{t=t_{0}}
$$

does not depend on the choice of $\left[q_{0}, \xi_{0}\right]_{G}$ as before, although, of course, it depends on the choice of $v_{0}$ and $q_{0}$, satisfying $\tau\left(v_{0}\right)=q_{0}$. Then, we have

$$
\left.\frac{D^{(A, V)} v_{0}(t)}{D t}\right|_{t=t_{0}}=\left.\frac{D v_{0}(t)}{D t}\right|_{t=t_{0}}-\left.\frac{D v_{0}^{h}(t)}{D t}\right|_{t=t_{0}} \text {. }
$$

It is clear that the right-hand side does not depend on the choice of the curve $g_{0}(t)$ satisfying $g_{0}\left(t_{0}\right)=e$ and $\dot{g}_{0}\left(t_{0}\right)=\xi_{0}$.

Now we claim that $\left[D v_{0}(t) / D t\right]_{G}$ depends only on the curve $[v]_{G}(t)$ in $V / G$ and on $\left[q_{0}, \xi_{0}\right]_{G} \in \tilde{\mathfrak{g}}_{x_{0}}$ but not on the particular choice of $v_{0}$ and $q_{0}$ satisfying the previous assumptions. Since we already know that $\left[D^{(A, H)} v_{0}(t) / D t\right]_{G}$ depends only on the curve $[v]_{G}(t)$ on $V / G$, we will then conclude, by definition of $\left[D^{(A, V)} v_{0}(t) / D t\right]_{G}$, that $\left[D^{(A, V)} v_{0}(t) / D t\right]_{G}$ depends only on the curve $[v]_{G}(t)$ in $V / G$ and on $\left[q_{0}, \xi_{0}\right]_{G} \in$ $\tilde{\mathfrak{g}}_{x_{0}}$ but not on the particular choice of $v_{0}$ and $q_{0}$ satisfying the previous assumptions.

To prove the claim, choose $v_{1} \in\left[v_{0}\right]_{G}$ and also $q_{1} \in\left[q_{0}\right]_{G}$ such that $\tau\left(v_{1}\right)=q_{1}$. Then there exists $h \in G$ such that $q_{1}=h q_{0}$ and $\xi_{1}=\operatorname{Ad}_{h} \xi_{0}$, and we also know from Lemma 5.2.1 that $v_{1}=h v_{0}$. If a curve $g_{0}(t)$ on $G$ satisfies $g_{0}\left(t_{0}\right)=e$ and $\dot{g}_{0}\left(t_{0}\right)=\xi_{0}$, let $g_{1}(t)=h g_{0}(t) h^{-1}$, which satisfies, $g_{1}\left(t_{0}\right)=e$ and $\dot{g}_{1}\left(t_{0}\right)=\xi_{1}$. Lemma 5.2.1 shows that there is a unique curve $v_{q_{1}}^{h}(t)$ in $V$ such that $\left[v_{q_{1}}^{h}(t)\right]_{G}=$ $[v]_{G}(t)$ and $\tau\left(v_{q_{1}}^{h}(t)\right)=x_{q_{1}}^{h}(t)$ for all $t$. Define $v_{1}(t)=g_{1}(t) v_{q_{1}}^{h}(t)$ for all $t$ and also $q_{1}(t)=g_{1}(t) x_{q_{1}}^{h}(t)$. Then we see that $v_{1}(t)=h g_{0}(t) h^{-1} h v_{0}=h v_{0}(t)$. Lemma 5.2.1 implies that $v_{1}(t)$ is the only curve in $V$ such that $\left[v_{1}(t)\right]_{G}=[v]_{G}(t)$ and $\tau\left(v_{1}(t)\right)=q_{1}(t)$ for all $t$. Then using Lemma 6.1.11, we obtain

$$
\left[\left.\frac{D v_{1}(t)}{D t}\right|_{t=t_{0}}\right]_{G}=\left[\left.h \frac{D v_{0}(t)}{D t}\right|_{t=t_{0}}\right]_{G}=\left[\left.\frac{D v_{0}(t)}{D t}\right|_{t=t_{0}}\right]_{G} .
$$


This and the definition of the vertical covariant derivative shows that

$$
\left[\left.\frac{D^{(A, V)} v_{1}(t)}{D t}\right|_{t=t_{0}}\right]_{G}=\left[\left.h \frac{D^{(A, V)} v_{0}(t)}{D t}\right|_{t=t_{0}}\right]_{G}=\left[\left.\frac{D^{(A, V)} v_{0}(t)}{D t}\right|_{t=t_{0}}\right]_{G} .
$$

This argument also shows that we can define the quotient covariant derivative of the curve $[v]_{G}(t)$ in $V / G$ with respect to $\left[q_{0}, \xi_{0}\right]_{G}$ by

$$
\left.\left[\frac{D^{(A)}}{D t}\right]_{G,\left[q_{0}, \xi_{0}\right]_{G}}[v]_{G}(t)\right|_{t=t_{0}}=\left[\left.\frac{D v_{0}(t)}{D t}\right|_{t=t_{0}}\right]_{G} .
$$

This justifies the following definition of the reduced covariant derivative and also the reduced vertical covariant derivative.

Definition 6.2.2. Assume the same conditions as in Definition 6.1 .9 and assume that the connection $\nabla$, and therefore also the covariant derivative $D / D t$, is $G$ invariant. Let $[v]_{G}(t)$ be a given curve in $V / G$. Let $(\tau / G)[v]_{G}\left(t_{0}\right)=\left[q_{0}\right]_{G}$ and choose $\left[q_{0}, \xi_{0}\right]_{G} \in \tilde{\mathfrak{g}}_{x_{0}}$. Let $v_{q_{0}}^{h}(t)$ be the unique curve in $V$ such that $\left[v_{q_{0}}^{h}(t)\right]_{G}=[v]_{G}(t)$ and $\tau\left(v_{q_{0}}^{h}(t)\right)=x_{q_{0}}^{h}(t)$ for all $t$. Choose a curve $g_{0}(t)$ on $G$ such that $g_{0}\left(t_{0}\right)=e$ and $\dot{g}_{0}\left(t_{0}\right)=\xi_{0}$. Let $v_{0}(t)=g_{0}(t) v_{q_{0}}^{h}(t)$.

(a) The quotient, or reduced, covariant derivative of the curve $[v]_{G}(t)$ with respect to $\left[q_{0}, \xi_{0}\right]_{G}$ at $t=t_{0}$ is defined by

$$
\left.\left[\frac{D^{(A)}}{D t}\right]_{G,\left[q_{0}, \xi_{0}\right]_{G}}[v]_{G}(t)\right|_{t=t_{0}}=\left[\left.\frac{D v_{0}(t)}{D t}\right|_{t=t_{0}}\right]_{G}
$$

(b) The quotient, or reduced, vertical covariant derivative of the curve $[v]_{G}(t)$ with respect to $\left[q_{0}, \xi_{0}\right]_{G}$ at $t=t_{0}$ is defined by

$$
\left.\left[\frac{D^{(A, V)}}{D t}\right]_{G,\left[q_{0}, \xi_{0}\right]_{G}}[v]_{G}(t)\right|_{t=t_{0}}=\left[\left.\frac{D^{(A, V)} v_{0}(t)}{D t}\right|_{t=t_{0}}\right]_{G}
$$

The following formula is a direct consequence of the previous definition and previous equalities

$$
\begin{aligned}
& {\left.\left[\frac{D^{(A)}}{D t}\right]_{G,\left[q_{0}, \xi_{0}\right]_{G}}[v]_{G}(t)\right|_{t=t_{0}}} \\
& \quad=\left.\left[\frac{D^{(A, V)}}{D t}\right]_{G,\left[q_{0}, \xi_{0}\right]_{G}}[v]_{G}(t)\right|_{t=t_{0}}+\left.\left[\frac{D^{(A, H)}}{D t}\right]_{G}[v]_{G}(t)\right|_{t=t_{0}} .
\end{aligned}
$$

Next we consider an important particular case in which the reduced vertical covariant derivative can be calculated with a formula entirely similar to the one derived before for the case of a constant curve. 
Lemma 6.2.3. Assume the conditions of definition 6.2 .2 and, in addition, assume that there is a $G$-invariant section $u \in \Gamma^{G}(V)$ such that $v_{0}(t)=u\left(g_{0}(t) x_{q_{0}}^{h}(t)(t)\right)$. Then

$$
\left.\left[\frac{D^{(A, V)}}{D t}\right]_{G,\left[q_{0}, \xi_{0}\right]_{G}}[v]_{G}(t)\right|_{t=t_{0}}=\left[\left.\frac{D g_{0}(t) v_{0}}{D t}\right|_{t=t_{0}}\right]_{G} .
$$

Proof. The proof is a direct consequence of Lemma 6.1.10(c).

\section{Quotient Vertical Connections.}

Now we will describe the notion of quotient, or reduced, vertical connection and also the quotient, or reduced, connection.

Definition 6.2.4. Let $\left[q_{0}, \xi_{0}\right]_{G} \in \tilde{\mathfrak{g}}$ with $\tilde{\pi}_{G}\left[q_{0}, \xi_{0}\right]_{G}=\left[q_{0}\right]_{G}$ and let $[v]_{G} \in$ $\Gamma(V / G)$, where $v \in \Gamma^{G}(V)$ according to Lemma 5.2.2. Let $Y_{0} \in I_{G}^{V}(Q)$ such that $\beta_{A}\left(Y_{0}\right)=\left[q_{0}, \xi_{0}\right]_{G}$, according to Lemma 5.1.2. Then

(a) The quotient, or reduced, vertical connection is defined by

$$
\left[\nabla^{(A, V)}\right]_{G,\left[q_{0}, \xi_{0}\right]_{G}}[v]_{G}=\left[\nabla_{Y_{0}}^{(A, V)} v\right]_{G} .
$$

(b) Let $\bar{X}_{0} \in T_{\left[q_{0}\right]_{G}}(Q / G)$, define $X_{0}=\bar{X}_{0}^{h}$, so $X_{0} \in I_{G}^{H}(Q)$, and $Z_{0}=X_{0}+Y_{0}$. The quotient, or reduced, connection is defined by the condition

$$
\left[\nabla^{(A)}\right]_{G, \bar{X}_{0} \oplus\left[q_{0}, \xi_{0}\right]_{G}}[v]_{G}=\left[\nabla_{Z_{0}} v\right]_{G}
$$

or by the equivalent condition

$$
\left[\nabla^{(A)}\right]_{G, \bar{X}_{0} \oplus\left[q_{0}, \xi_{0}\right]_{G}}[v]_{G}=\left[\nabla^{(A, H)}\right]_{G, \bar{X}_{0}}[v]_{G}+\left[\nabla^{(A, V)}\right]_{G,\left[q_{0}, \xi_{0}\right]_{G}}[v]_{G}
$$

One sees that the expressions in Definition 6.2.4 are well defined using Lemma 6.1.11, which establishes invariance of $\nabla^{(A, V)}$ and $\nabla^{(A, H)}$, and also Lemma 5.2.2.

The following lemma establishes the link between the notions of quotient vertical covariant derivative (resp. quotient covariant derivative) and quotient vertical connection (resp. quotient connection).

Lemma 6.2.5. Let $\left[q_{0}, \xi_{0}\right]_{G} \in \tilde{\mathfrak{g}}$ and let $[v]_{G} \in \Gamma(V / G)$, where $v \in \Gamma^{G}(V)$ according to Lemma 5.2.2. Let $[q]_{G}(t)=x(t)$ be any curve in $Q / G$ such that $\tilde{\pi}_{G}\left(\left[q_{0}, \xi_{0}\right]_{G}\right)=[q]_{G}\left(t_{0}\right)$ and let, with a convenient abuse of notation, $[v]_{G}(t)=$ $[v]_{G}\left([q]_{G}(t)\right)$. Assume that $D / D t$ is $G$-invariant. Then we have

(a)

$$
\left.\left[\frac{D^{(A, V)}}{D t}\right]_{G,\left[q_{0}, \xi_{0}\right]_{G}}[v]_{G}\right|_{t=t_{0}}=\left[\nabla^{(A, V)}\right]_{G,\left[q_{0}, \xi_{0}\right]_{G}}[v]_{G}\left([q]_{G}\left(t_{0}\right)\right)
$$


(b)

$$
\left.\left[\frac{D^{(A)}}{D t}\right]_{G,\left[q_{0}, \xi_{0}\right]_{G}}[v]_{G}\right|_{t=t_{0}}=\left[\nabla^{(A)}\right]_{G, \dot{x}\left(t_{0}\right) \oplus\left[q_{0}, \xi_{0}\right]_{G}}[v]_{G}\left([q]_{G}\left(t_{0}\right)\right)
$$

Proof. The proof is a direct consequence of Lemma 6.2.3 and the fundamental link between covariant derivatives and connections in a vector bundle explained before (see the paragraph Affine Connections in $§ 2.3$ ).

\section{Group Actions on Lagrange-Poincaré Bundles.}

Now we shall define the notion of an action of a group $G$ on an object of $\mathfrak{L}$.

Definition 6.2.6. An action in the category $\mathfrak{L P}$ of a group $G$ on an object $T Q \oplus V$ of $\mathfrak{L P}$ is a vector bundle action $\rho: G \times T Q \oplus V \rightarrow T Q \oplus V$ such that, for each $g \in G, \rho_{g}: T Q \oplus V \rightarrow T Q \oplus V$ is an isomorphism of $\mathfrak{L P}$. Sometimes we will call an action in the category $\mathfrak{L P}$ simply an action, if the fact that it is an action in the category $\mathfrak{L P}$ is clear from the context.

Corollary 6.2.7. If $\rho: G \times T Q \oplus V \rightarrow T Q \oplus V$ is an action in the category $\mathfrak{L P}$ then we have

(i) $\rho_{*}: G \times \Gamma(T Q \oplus V) \rightarrow \Gamma(T Q \oplus V)$ is a representation of $G$ such that, for each $g \in G, \rho_{g *}: \Gamma(T Q \oplus V) \rightarrow \Gamma(T Q \oplus V)$ is a Lie algebra isomorphism;

(ii) The restriction $\rho \mid G \times T Q: G \times T Q \rightarrow T Q$ is the tangent lift of the action on the zero section, $\rho_{0}: G \times Q \rightarrow Q$.

Proof. (i) is a consequence of Lemma 6.1.5 and (ii) is a consequence of the definition of morphism in the category $\mathfrak{L P}$.

Definition 6.2.8. Let $\tau: V \rightarrow Q$ be a vector bundle and let $\rho: G \times V \rightarrow V$ be a vector bundle action. As usual, the action on the zero section $\rho_{0}: G \times Q \rightarrow Q$ is assumed to give $Q$ the structure of a principal bundle. Let $\iota_{G}(V): I_{G}(V) \rightarrow Q / G$ be the vector bundle whose fiber at the point $[q]_{G} \in Q / G$ is the space of all $G$-invariant sections of the restriction $V \mid G q$, where, as usual, $G q=[q]_{G}$ is the orbit of $q$. In addition, let $\gamma_{G}(V)$ be the vector bundle isomorphism $\gamma_{G}(V): I_{G}(V) \rightarrow V / G$ given by

$$
\gamma_{G}(V)\left(v_{[q]_{G}}\right)=\left[v_{[q]_{G}}(q)\right]_{G},
$$

where $v_{[q]_{G}}$ is a section of $V \mid G q$.

It is easy to see that $\gamma_{G}(V)$ is a well defined vector bundle isomorphism. Observe that Definition 6.2.8 is consistent with Definition 5.1.1; in fact, we can easily see that $I_{G}^{H}(T Q)=I_{G}(\operatorname{Hor}(T Q))$ and $I_{G}^{V}(T Q)=I_{G}(\operatorname{Ver}(T Q))$. Obviously we have a vector bundle isomorphism

$$
\gamma: I_{G}^{H}(T Q) \oplus I_{G}^{V}(T Q) \oplus I_{G}(V) \rightarrow \operatorname{Hor}(T Q) / G \oplus \operatorname{Ver}(T Q) / G \oplus(V / G)
$$


defined by

$$
\begin{aligned}
\gamma & \equiv \gamma_{G}(\operatorname{Hor}(T Q)) \oplus \gamma_{G}(\operatorname{Ver}(T Q)) \oplus \gamma_{G}(V) \\
& \equiv \gamma_{G}(\operatorname{Hor}(T Q) \oplus \operatorname{Ver}(T Q) \oplus V)
\end{aligned}
$$

\section{Isomorphisms Between Quotient Bundles.}

Using the definition of $\beta_{A}$ from Lemma 5.1.2, taking into account the natural identifications $T Q \equiv \operatorname{Hor}(T Q) \oplus \operatorname{Ver}(T Q)$ and also $T Q / G \equiv \operatorname{Hor}(T Q) / G \oplus \operatorname{Ver}(T Q) / G$, and using the isomorphism $\gamma_{G}(V)$ just defined, we obtain a vector bundle isomorphism

$$
\beta_{A} \oplus \gamma_{G}(V): I_{G}^{H}(T Q) \oplus I_{G}^{V}(T Q) \oplus I_{G}(V) \rightarrow T(Q / G) \oplus \tilde{\mathfrak{g}} \oplus(V / G) .
$$

Also from Lemma 5.2.2 we see that there are linear isomorphisms

$$
\begin{gathered}
\pi_{G}(V)^{*}: \Gamma(V / G) \rightarrow \Gamma^{G}(V), \\
\pi_{G}(\operatorname{Hor}(T Q))^{*}: \Gamma(\operatorname{Hor}(T Q) / G) \rightarrow \Gamma^{G}(\operatorname{Hor}(T Q)),
\end{gathered}
$$

and

$$
\pi_{G}(\operatorname{Ver}(T Q))^{*}: \Gamma(\operatorname{Ver}(T Q) / G) \rightarrow \Gamma^{G}(\operatorname{Ver}(T Q)) .
$$

Definition 6.2.9. Define the linear isomorphism

$$
\begin{aligned}
\pi_{*}: \Gamma^{G}(\operatorname{Hor}(T Q)) \oplus \Gamma^{G}(\operatorname{Ver}(T Q)) \oplus \Gamma^{G}(V) \\
\rightarrow \Gamma(\operatorname{Hor}(T Q) / G) \oplus \Gamma(\operatorname{Ver}(T Q) / G) \oplus \Gamma(V / G)
\end{aligned}
$$

by

$$
\pi_{*}=\left(\pi_{G}(\operatorname{Hor}(T Q))^{*} \oplus \pi_{G}(\operatorname{Ver}(T Q))^{*} \oplus \pi_{G}(V)^{*}\right)^{-1} .
$$

Similarly, the linear isomorphism

$$
\begin{aligned}
\varphi \equiv \varphi_{A}(Q, G, V) \equiv \varphi_{A}: \Gamma^{G}(\operatorname{Hor}(T Q)) \oplus \Gamma^{G}(\operatorname{Ver}(T Q)) \oplus \Gamma^{G}(V) \\
\rightarrow \Gamma(T(Q / G)) \oplus \Gamma(\tilde{\mathfrak{g}}) \oplus \Gamma(V / G)
\end{aligned}
$$

is defined by

$$
\varphi=\left(\beta_{A} \oplus \gamma_{G}(V)\right)_{*} \circ \gamma^{*} \circ \pi_{*} .
$$

If we consider $\gamma^{*} \circ \pi_{*}$ as being an identification, which is natural in the present context, we can write $\varphi \equiv\left(\beta_{A} \oplus \gamma_{G}(V)\right)_{*}$. Likewise, if we consider $\gamma^{*}$ and $\pi_{*}$ as being identifications we can write the map

$$
\varphi: \Gamma(T Q / G) \oplus \Gamma(V / G) \rightarrow \Gamma(T(Q / G) \oplus \tilde{\mathfrak{g}}) \oplus \Gamma(V / G)
$$

as $\varphi \equiv \alpha_{A}^{*} \oplus \operatorname{id}_{(V / G) *}$. 


\section{Structure of Reduced Lagrange-Poincaré Bundles.}

Now we state and prove our main result in this section.

Theorem 6.2.10. Let $\tau_{Q} \oplus \tau: T Q \oplus V \rightarrow Q$ be an object of $\mathfrak{L P}$ and let $\nabla, \omega$, [,] be the structure on $V$. Let $\rho: G \times(T Q \oplus V) \rightarrow T Q \oplus V$ be an action in the category $\mathfrak{L P}$. Choose a connection $A$ on the principal bundle $Q$ with structure group G. Consider the bundle

$$
\begin{aligned}
& T(Q / G) \oplus \tilde{\mathfrak{g}} \oplus(V / G) . \\
& \text { Define the structure } \nabla^{\tilde{\mathfrak{g}}}, \omega^{\tilde{\mathfrak{g}}},[,]^{\tilde{\mathfrak{g}}} \text { on } \tilde{\mathfrak{g}} \oplus(V / G) \text { by } \\
& \nabla_{X}^{\tilde{\mathfrak{g}}}\left(\bar{\xi} \oplus[v]_{G}\right)=\tilde{\nabla}_{X}^{A} \bar{\xi} \oplus\left[\nabla^{(A, H)}\right]_{G, X}[v]_{G}-[\omega]_{G}(X, \bar{\xi}) \\
& \omega^{\tilde{\mathfrak{g}}}\left(X_{1}, X_{2}\right)=\tilde{B}^{A}\left(X_{1}, X_{2}\right) \oplus[\omega]_{G}\left(X_{1}, X_{2}\right) \\
& {\left[\bar{\xi}_{1} \oplus\left[v_{1}\right]_{G}, \bar{\xi}_{2} \oplus\left[v_{2}\right]_{G}\right]^{\tilde{\mathfrak{g}}}=\left[\bar{\xi}_{1}, \bar{\xi}_{2}\right] \oplus\left[\nabla^{(A, V)}\right]_{G, \bar{\xi}_{1}}\left[v_{2}\right]_{G}-\left[\nabla^{(A, V)}\right]_{G, \bar{\xi}_{2}}\left[v_{1}\right]_{G}} \\
& -[\omega]_{G}\left(\bar{\xi}_{1}, \bar{\xi}_{2}\right)+\left[\left[v_{1}\right]_{G},\left[v_{2}\right]_{G}\right] .
\end{aligned}
$$

Then the bundle $T(Q / G) \oplus \tilde{\mathfrak{g}} \oplus(V / G)$, with the structure on the bundle $\tilde{\mathfrak{g}} \oplus(V / G)$ given by $\nabla^{\tilde{\mathfrak{g}}}, \omega^{\tilde{\mathfrak{g}}},[,]^{\tilde{\mathfrak{g}}}$, is an object of the category $\mathfrak{L} \mathfrak{P}$.

\section{Remark.}

Theorems 6.2.10 and 5.2.4 seem to shed light on the structure of Lie group extensions, namely that they necessarily have the structure, roughly speaking, of a semidirect product with a curvature cocycle. It would be of interest to explore this technical point further.

Proof of the Theorem.. It is easy to see that $\nabla^{\tilde{\mathfrak{g}}}$ is a connection on $\tilde{\mathfrak{g}} \oplus(V / G)$ (see the paragraph Affine Connections of $\$ 2.3$ for the definition). It is also easy to check that $\omega^{\tilde{\mathfrak{g}}}$ is a $\tilde{\mathfrak{g}} \oplus(V / G)$-valued 2 -form. We must now show that the expression of $[,]^{\tilde{\mathfrak{g}}}$ given in the statement of the theorem defines a Lie bracket on the fibers of $\tilde{\mathfrak{g}} \oplus(V / G)$ that endows this bundle with the structure of a Lie algebra bundle. It is clear that each one of the terms $\left[\bar{\xi}_{1}, \bar{\xi}_{2}\right],-[\omega]_{G}\left(\bar{\xi}_{1}, \bar{\xi}_{2}\right)$ and $\left.\left[\left[v_{1}\right]_{G},\left[v_{2}\right]_{G}\right]\right]$ defines a bilinear and skew symmetric operation on the fibers of $\tilde{\mathfrak{g}} \oplus(V / G)$. Now using Lemma 6.1.10 and Definition 6.2.4 we can see that the term

$$
\left[\nabla^{(A, V)}\right]_{G, \bar{\xi}_{1}}\left[v_{2}\right]_{G}-\left[\nabla^{(A, V)}\right]_{G, \bar{\xi}_{2}}\left[v_{1}\right]_{G}
$$

also defines a bilinear and skew symmetric operation on the fibers of $\tilde{\mathfrak{g}} \oplus(V / G)$. So far we have proven that the expression of $[,]^{\tilde{\mathfrak{g}}}$ defines a bilinear and skew symmetric operation on the fibers of $\tilde{\mathfrak{g}} \oplus(V / G)$. Moreover, it is easy to see that for any function $f \in C^{\infty}(Q / G)$ we have

$$
\left[\bar{\xi}_{1} \oplus\left[v_{1}\right]_{G}, f\left(\bar{\xi}_{2} \oplus\left[v_{2}\right]_{G}\right)\right]^{\tilde{\mathfrak{g}}}=f\left[\bar{\xi}_{1} \oplus\left[v_{1}\right]_{G}, \bar{\xi}_{2} \oplus\left[v_{2}\right]_{G}\right]^{\tilde{\mathfrak{g}}} .
$$

It remains to prove that $[,]^{\tilde{\mathfrak{g}}}$ satisfies the Jacobi identity. For this we must first study the Lie algebra structure on the space of sections

$$
\Gamma(T(Q / G)) \oplus \Gamma(\tilde{\mathfrak{g}}) \oplus \Gamma(V / G) \equiv \Gamma((T(Q / G)) \oplus \tilde{\mathfrak{g}} \oplus(V / G)) .
$$


According to Definition 6.1.1 the operation on sections $Z_{i} \oplus v_{i} \in \Gamma(T Q \oplus V)$, $i=1,2$, given by

$$
\left[Z_{1} \oplus v_{1}, Z_{2} \oplus v_{2}\right]=\left[Z_{1}, Z_{2}\right] \oplus \nabla_{Z 1} v_{2}-\nabla_{Z 2} v_{1}-\omega\left(Z_{1}, Z_{2}\right)+\left[v_{1}, v_{2}\right]
$$

is a Lie bracket.

The $G$-invariance of $\nabla, \omega$, and [,] implies that

$$
\Gamma^{G}(T Q \oplus V) \equiv \Gamma^{G}(\operatorname{Hor}(T Q)) \oplus \Gamma^{G}(\operatorname{Ver}(T Q)) \oplus \Gamma^{G}(V)
$$

is a Lie subalgebra. Using this and the linear isomorphism $\varphi$ introduced in Definition 6.2.9, we can define a Lie algebra on the space of sections

$$
\Gamma(T(Q / G)) \oplus \Gamma(\tilde{\mathfrak{g}}) \oplus \Gamma(V / G)
$$

by

$$
\begin{array}{r}
{\left[X_{1} \oplus \bar{\xi}_{1} \oplus\left[v_{1}\right]_{G}, X_{2} \oplus \bar{\xi}_{2} \oplus\left[v_{2}\right]_{G}\right]=\varphi\left[\varphi^{-1}\left(X_{1} \oplus \bar{\xi}_{1} \oplus\left[v_{1}\right]_{G}\right),\right.} \\
\left.\varphi^{-1}\left(X_{2} \oplus \bar{\xi}_{2} \oplus\left[v_{2}\right]_{G}\right)\right] .
\end{array}
$$

We now show that this Lie bracket coincides with the one constructed on

$$
\Gamma(T(Q / G)) \oplus \Gamma(\tilde{\mathfrak{g}}) \oplus \Gamma(V / G)
$$

using the structures $\nabla^{\tilde{\mathfrak{g}}}, \omega^{\tilde{\mathfrak{g}}}$ and [, $]^{\tilde{\mathfrak{g}}}$, according to Definition 6.1.1. We know that $\varphi^{-1} X_{i}=X_{i}^{h}$, for $i=1,2$. Let $\varphi^{-1} \bar{\xi}_{i}=Y_{i}$ and $\varphi^{-1}\left[v_{i}\right]_{G}=v_{i}$, for $i=1,2$ (recall that there is a unique $v_{i} \in \Gamma^{G}(V)$ in each class $\left[v_{i}\right]_{G}$ ). By Definition 6.1.1 we have

$$
\begin{aligned}
& {\left[X_{1}^{h} \oplus Y_{1} \oplus v_{1}, X_{2}^{h} \oplus Y_{2} \oplus v_{2}\right]} \\
& \quad=\left[X_{1}^{h}+Y_{1}, X_{2}^{h}+Y_{2}\right] \oplus \nabla_{X_{1}^{h}+Y_{1}} v_{2}-\nabla_{X_{2}^{h}+Y_{2}} v_{1} \\
& \quad-\omega\left(X_{1}^{h}+Y_{1}, X_{2}^{h}+Y_{2}\right)+\left[v_{1}, v_{2}\right] .
\end{aligned}
$$

Since $\varphi\left|\Gamma^{G}(T Q)=\alpha_{A *}\right| \Gamma^{G}(T Q)$ we have, using Theorem 5.2.4, that

$$
\varphi\left[X_{1}^{h} \oplus Y_{1}, X_{2}^{h} \oplus Y_{2}\right]=\left[X_{1}, X_{2}\right] \oplus \tilde{\nabla}_{X_{1}}^{A} \bar{\xi}_{2}-\tilde{\nabla}_{X_{2}}^{A} \bar{\xi}_{1}-\tilde{B}^{A}\left(X_{1}, X_{2}\right)+\left[\bar{\xi}_{1}, \bar{\xi}_{2}\right] .
$$

Using Definition 6.2.4 we have, for

$$
\varphi\left(\nabla_{X_{i}^{h}+Y_{i}} v_{j}\right)=\left[\nabla^{(A, H)}\right]_{G, X_{i}}\left[v_{j}\right]_{G}+\left[\nabla^{(A, V)}\right]_{G, \bar{\xi}_{i}}\left[v_{j}\right]_{G}
$$

Using Lemma 6.1 .7 we can easily see that

$$
\begin{aligned}
\varphi \omega\left(X_{1}^{h}+\right. & \left.Y_{1}, X_{2}^{h}+Y_{2}\right) \\
& =[\omega]_{G}\left(X_{1}, X_{2}\right)+[\omega]_{G}\left(X_{1}, \bar{\xi}_{2}\right)+[\omega]_{G}\left(\bar{\xi}_{1}, X_{2}\right)+[\omega]_{G}\left(\bar{\xi}_{1}, \bar{\xi}_{2}\right)
\end{aligned}
$$

and also that

$$
\varphi\left[v_{1}, v_{2}\right]=\left[\left[v_{1}\right]_{G},\left[v_{2}\right]_{G}\right] .
$$


It is not difficult to conclude that the operation on

$$
\Gamma((T(Q / G)) \oplus \tilde{\mathfrak{g}} \oplus(V / G))
$$

using the structures $\nabla^{\tilde{\mathfrak{g}}}, \omega^{\tilde{\mathfrak{g}}}$, and [, ] $]^{\tilde{\mathfrak{g}}}$, according to Definition 6.1.1 is a Lie bracket. Moreover, we have a description of this bracket using the isomorphism $\varphi$. It follows that the restriction of this Lie bracket to $\Gamma(\tilde{\mathfrak{g}} \oplus(V / G))$ is also a Lie bracket. This restriction is given by $[,]^{\tilde{\mathfrak{g}}}$. Therefore, the Jacobi identity for the bilinear and skew symmetric operation on fibers of $\Gamma(\tilde{\mathfrak{g}} \oplus(V / G))$ defined by $[,]^{\tilde{\mathfrak{g}}}$ is satisfied. This finishes the proof of the theorem.

Now we introduce some more notation.

Definition 6.2.11. Assume the hypothesis of Theorem 6.2.10. The bundle

$$
T(Q / G) \oplus \tilde{\mathfrak{g}} \oplus(V / G)
$$

with the reduced structure $\nabla^{\tilde{\mathfrak{g}}}$, $\omega^{\tilde{\mathfrak{g}}}$, and $[,]^{\tilde{\mathfrak{g}}}$ on $\tilde{\mathfrak{g}} \oplus(V / G)$ is called the reduced bundle with respect to the group $G$ and the connection $A$. The reduced Lie algebra structure on

$$
\Gamma(T(Q / G) \oplus \tilde{\mathfrak{g}} \oplus(V / G))
$$

is the one described in Theorem 6.2 .10 using the reduced structure $\nabla^{\tilde{\mathfrak{g}}}, \omega^{\tilde{\mathfrak{g}}},[,]^{\tilde{\mathfrak{g}}}$ on $\tilde{\mathfrak{g}} \oplus(V / G)$ according to Definition 6.1.1.

Let $W=T Q \oplus V$, for short. We will denote by $\alpha_{A}^{W}$ the natural map

$$
\alpha_{A}^{W}:(T Q \oplus V) / G \rightarrow T(Q / G) \oplus \tilde{\mathfrak{g}} \oplus(V / G),
$$

that is, $\alpha_{A}^{W}:=\alpha_{A} \oplus \mathrm{id}_{V / G}$, where $\alpha_{A}$ is the map of Lemma 2.4.2. To make the notation consistent in the case $V=0$, that is, $W=T Q$, we will write $\alpha_{A} \equiv \alpha_{A}^{T Q}$.

Lemma 6.2.12. Assume the hypothesis of Theorem 6.2.10. Then we have:

(a) The push forward map

$$
\alpha_{A *}^{W}: \Gamma((T Q \oplus V) / G) \rightarrow \Gamma(T(Q / G) \oplus \tilde{\mathfrak{g}} \oplus(V / G))
$$

is a Lie algebra isomorphism. Here the Lie algebra on $\Gamma((T Q \oplus V) / G)$ is the natural quotient Lie algebra structure, as defined in the comments following Lemma 5.2.2.

(b) Let $A^{\prime}$ be another connection on the principal bundle $Q$ with structure group $G$. Then $\alpha_{A^{\prime}}^{W} \circ\left(\alpha_{A}^{W}\right)^{-1}$ is an isomorphism in the category $\mathfrak{L P}$ if and only if $A=A^{\prime}$.

Proof. (a) The proof of this part is an easy consequence of the proof of Theorem 6.2.10.

(b) Let

$$
X \equiv X \oplus 0 \oplus 0 \in \Gamma(T(Q / G) \oplus \tilde{\mathfrak{g}} \oplus(V / G))
$$


The proof of Theorem 6.2.10 shows that $\left(\alpha_{A}^{W}\right)^{-1}(X)$ is the horizontal lift of $X$ with respect to the connection $A$ and hence $\left(\alpha_{A^{\prime}}^{W} \circ\left(\alpha_{A}^{W}\right)^{-1}\right)(X)$ can be written as

$$
\left(\alpha_{A^{\prime}}^{W} \circ\left(\alpha_{A}^{W}\right)^{-1}\right)(X)=X \oplus \bar{\xi},
$$

where $\bar{\xi} \in \tilde{\mathfrak{g}}$. Also, by the same argument as in the proof of Theorem 6.2.10, we can see that $\bar{\xi}=0$ if and only if $\left(\alpha_{A}^{W}\right)^{-1}(X)$ is horizontal with respect to the connection $A^{\prime}$. On the other hand, the proof of Theorem 6.2.10, also shows that for any

$$
\bar{\xi} \oplus[v]_{G} \equiv 0 \oplus \bar{\xi} \oplus[v]_{G} \in \Gamma(T(Q / G) \oplus \tilde{\mathfrak{g}} \oplus(V / G))
$$

we have

$$
\left(\alpha_{A^{\prime}}^{W} \circ\left(\alpha_{A}^{W}\right)^{-1}\right)\left(\bar{\xi} \oplus[v]_{G}\right) \in \Gamma(\tilde{\mathfrak{g}} \oplus(V / G)) .
$$

Using what we have proven so far, we can deduce that the conditions

$$
\left(\alpha_{A^{\prime}}^{W} \circ\left(\alpha_{A}^{W}\right)^{-1}\right) T(Q / G)=T(Q / G)
$$

and

$$
\left(\alpha_{A^{\prime}}^{W} \circ\left(\alpha_{A}^{W}\right)^{-1}\right)(\tilde{\mathfrak{g}} \oplus(V / G))=\tilde{\mathfrak{g}} \oplus(V / G)
$$

are satisfied if and only if $A=A^{\prime}$.

\subsection{Reduction by Stages of objects of $\mathfrak{L P}$}

We shall begin by recollecting, without proof, some basic facts. In this section $G$ will be a Lie group and $N \subset G$ a normal Lie subgroup.

Lemma 6.3.1. Assume that a group $G$ acts on the left on a manifold $Q$ (this time we do not need to assume that, with this action, $Q$ becomes a principal bundle) and let $N \subset G$ be a normal subgroup. Then the rule $[g]_{N}[q]_{N}=[g q]_{N}$ defines an action of $G / N$ on $Q / N$. The map

$$
i_{G / N}^{Q}: Q / G \rightarrow(Q / N) /(G / N)
$$

given by

$$
i_{G / N}^{Q}\left([q]_{G}\right)=\left[[q]_{N}\right]_{(G / N)}
$$

is a bijection.

If the action of $G$ on $Q$ is free then the action of $N$ on $Q$ is free and also the action of $G / N$ on $Q / N$ is free. Conversely, if the action of $N$ on $Q$ is free and also the action of $G / N$ on $Q / N$ is free then the action of $G$ on $Q$ is free.

Now assume, in addition, that $\pi: Q \rightarrow Q / G$, is a principal bundle with structure group $G$. Then the map $i_{G / N}^{Q}$ is a well defined diffeomorphism. Moreover, $Q / N$ is a principal bundle with structure group $G / N$ and $Q$ is a principal bundle with structure group $N$. Conversely, if $Q / N$ is a principal bundle with structure group $G / N$ and $Q$ is a principal bundle with structure group $N$ then $Q$ is a principal bundle with structure group $G$. 
We are interested in cases of the previous lemma in which the manifold $Q$ carries some extra structures which remain invariant under the action of the group $G$ and we also want to show how to obtain the corresponding quotient structures by stages. The next lemma considers the case of an invariant vector bundle structure. We will use some notation and results described in section $\S 5.2$, in particular Lemma 5.2.1

Lemma 6.3.2. Let $\tau: V \rightarrow Q$ be a vector bundle and let $\rho: G \times V \rightarrow V$ be a vector bundle action of the group $G$ on $V$ covering the action $\rho_{0}: G \times Q \rightarrow Q$ on the zero section. We assume that the action $\rho_{0}$ endows $Q$ with the structure of a principal bundle over $Q / G$. Let $N \subset G$ be a normal subgroup. Then

$$
\tau / N: V / N \rightarrow Q / N \quad \text { and } \quad \tau / G: V / G \rightarrow Q / G
$$

are vector bundles and $G / N$ acts with a vector bundle action on $V / N$. The quotient

$$
(\tau / N) /(G / N):(V / N) /(G / N) \rightarrow(Q / N) /(G / N)
$$

is a vector bundle. The isomorphism $i_{G / N}^{V}$ is a vector bundle isomorphism.

Now we state the following lemma whose proof is straightforward.

Lemma 6.3.3. Assume the hypothesis of Lemma 6.1 .7 and, in addition, assume that $N$ is a normal subgroup of $G$. Thus, in particular, any one of the structures (a) or (b) defined in Lemma 6.1 .7 which is invariant under the action of $G$ is also invariant under the restricted action of $N$, so it gives rise to a corresponding quotient structure on the quotient vector bundle $V / N$. Then the quotient structures so defined are invariant under the action of $G / N$ on $V / N$ described in Lemma 6.3 .2 and they define corresponding quotient structures on the bundle $(V / N) /(G / N)$. Moreover, the isomorphism $i_{G / N}^{V}$ described in Lemma 6.3 .2 commutes with those structures, that is, it is also an isomorphism with respect to these structures.

Let us now consider an important example of this situation. Assume that the hypothesis of Lemma 6.3.1 hold. Let $\tilde{\mathfrak{n}}$ be as in Definition 2.3.3. Then $G / N$ acts on $\tilde{\mathfrak{n}}$ as follows:

$$
[g]_{N}[q, \xi]_{N}=\left[g q, \operatorname{Ad}_{g} \xi\right]_{N} .
$$

We will prove that this action is well defined. Since $N \subset G$ is a normal subgroup, for any $g \in G$ and any $n \in N$ there exist $n_{g}^{\prime}, n_{g}^{\prime \prime} \in N$ such that $g n=n_{g}^{\prime} g$ and $n g=g n_{g}^{\prime \prime}$. Besides, for any $\xi \in \mathfrak{n}$ we have $\operatorname{Ad}_{g} \xi \in \mathfrak{n}$. Now let $n_{i} \in N$ be given for $i=1,2$. Then we have

$$
\begin{aligned}
{\left[n_{1} g\right]_{N}\left[n_{2} q, \operatorname{Ad}_{n_{2}} \xi\right]_{N} } & =\left[n_{1} g n_{2} q, \operatorname{Ad}_{n_{1} g} \operatorname{Ad}_{n_{2}} \xi\right]_{N} \\
& =\left[n_{1} n_{2 g}^{\prime} g q, \operatorname{Ad}_{n_{1} n_{2 g}^{\prime} g} \xi\right]_{N} \\
& =\left[n_{1} n_{2 g}^{\prime} g q, \operatorname{Ad}_{n_{1} n_{2 g}^{\prime}} \operatorname{Ad}_{g} \xi\right]_{N} \\
& =\left[g q, \operatorname{Ad}_{g} \xi\right]_{N},
\end{aligned}
$$

which shows that the action is well defined.

Now we will study invariance properties of the structures on $\tilde{\mathfrak{n}}$. We will omit the proof of the next lemma, which is straightforward. 
Lemma 6.3.4. Assume the same hypothesis as in Lemma 6.3 .3 and, in addition, choose a connection $A_{N}$ on the principal bundle $Q$ with structure group $N$. Then the action of $G / N$ on $\tilde{\mathfrak{n}}$ defined above commutes with the Lie algebra structure on fibers of $\tilde{\mathfrak{n}}$ defined in Lemma 2.3 .5 and also with the $\tilde{\mathfrak{n}}$-valued curvature 2-form $\tilde{B}^{A_{N}}$ defined by equation (3.1.1). More precisely, for any $\left[q, \xi_{i}\right]_{N} \in \mathfrak{\mathfrak { n }}, i=1,2$, any $X_{i} \in T_{[q]_{N}}(Q / N), i=1,2$, and any $g \in G$ we have

$$
\left[[g]_{N}\left[q, \xi_{1}\right]_{N},[g]_{N}\left[q, \xi_{2}\right]_{N}\right]=[g]_{N}\left[\left[q, \xi_{1}\right]_{N},\left[q, \xi_{2}\right]_{N}\right]
$$

and

$$
\tilde{B}^{A_{N}}\left([g]_{N} X_{1},[g]_{N} X_{2}\right)=[g]_{N} \tilde{B}^{A_{N}}\left(X_{1}, X_{2}\right) .
$$

It is not generally true that for any connection $A_{N}$ the covariant derivative $\tilde{D}^{A_{N}} / D t$ or, equivalently, the connection $\tilde{\nabla}^{A_{N}}$ on the bundle $\tilde{\mathfrak{n}}$, is invariant under the action of $G / N$. However there is always a connection $A_{N}$ having this property, that is, having the property that the action of $G / N$ commutes with $\tilde{D}^{A_{N}} / D t$ or, equivalently, with the connection $\tilde{\nabla}^{A_{N}}$, as we shall see next. Let $\langle$,$\rangle be any G$ invariant metric on $Q$. It is not difficult to show that any given principal bundle, say $\pi: Q \rightarrow Q / G$ with structure group $G$, carries an invariant Riemannian metric. For instance, it is well known that using partitions of unit one can construct both, a Riemannian metric $g$ on the base $Q / G$ and also a principal connection $A$ on the principal bundle $Q$. With respect to the connection $A$, we have the decomposition $T Q=$ Hor $T Q \oplus \operatorname{Ver} T Q$. We are going to define an invariant metric $g^{h}$ on the vector bundle Hor $T Q$ and an invariant metric $g^{v}$ on the vector bundle Ver $T Q$. Then, by declaring that Hor $T Q$ and Ver $T Q$ are orthogonal, $g=g^{h} \oplus g^{v}$ will be an invariant positive definite metric on the vector bundle $T Q$, that is, an invariant Riemannian metric on $Q$. We can define $g^{h}$ as being the horizontal lift of the Riemannian metric $g$ on the base. To define $g^{v}$, let us recall that the vector bundle $\operatorname{Ver} T Q$ is isomorphic to the vertical invariant bundle $I_{G}^{V}(T Q)$, defined in 5.1.1. It is also well known that any vector bundle carries a positive definite metric, so let $\bar{g}^{v}$ be a positive definite metric on the vector bundle $I_{G}^{V}(T Q)$. Define the metric $g^{v}$ on $\operatorname{Ver} T Q$ as follows: For each $X_{q}, Y_{q} \in \operatorname{Ver} T Q$, let $X, Y \in I_{G}^{V}(T Q)$ be such that $X(q)=X_{q}$ and $Y(q)=Y_{q}$, then set, by definition, $g^{v}\left(X_{q}, Y_{q}\right)=\bar{g}^{v}(X, Y)$.

Then for each $q \in Q$ we have a direct sum orthogonal decomposition

$$
T_{q} Q=\operatorname{Ver}^{N}\left(T_{q} Q\right)+H_{N}(q),
$$

where $H_{N}(q)$ is the orthogonal complement of $\operatorname{Ver}^{N}\left(T_{q} Q\right)$. Given $v_{q} \in T_{q} Q$ we can then write $v_{q}=\xi q+v_{q H_{N}}$ where $v_{q H_{N}} \in H_{N}(q)$ and $\xi \in \mathfrak{n}$. We can easily see that the restriction

$$
T_{q} \pi_{N} \mid H_{N}(q): H_{N}(q) \rightarrow T_{[q]_{N}}(Q / N)
$$

is a linear isomorphism and that the collection of all $H_{N}(q)$ is $G$-invariant, therefore, also $N$-invariant. Thus the collection of all $H_{N}(q)$ defines a connection on the principal bundle $Q$ with structure group $N$. Let $A_{N}$ be the corresponding connection 1-form. By definition, we have $A_{N}\left(v_{q}\right)=\xi$ if $v_{q}=\xi q+v_{q H_{N}}$. The horizontal spaces of this connection are $\operatorname{Hor}_{q}^{A_{N}}(T Q) \equiv H_{N}(q)$. 
Lemma 6.3.5. Let $A_{N}$ be the connection associated to a $G$-invariant metric $\langle$, as explained above. Then for any $g \in G$ and any $v_{q} \in T_{q} Q$ we have $A_{N}\left(g v_{q}\right)=$ $\operatorname{Ad}_{g} A_{N}\left(v_{q}\right)$.

Proof. For any $g \in G$ and any $v_{q} \in T_{q} Q$ we have

$$
A_{N}\left(g v_{q}\right)=A_{N}\left(g \xi q+g v_{q H_{N}}\right)=A_{N}(g \xi q)+A_{N}\left(g v_{q H_{N}}\right) .
$$

Since the collection of horizontal spaces is $G$-invariant, it follows that $g v_{q H_{N}} \in$ $H_{N}(g q)$ and therefore $A_{N}\left(g v_{q H_{N}}\right)=0$. On the other hand we have $A_{N}(g \xi q)=$ $A_{N}\left(\operatorname{Ad}_{g} \xi g q\right)$. Since $N$ is normal, we have $\operatorname{Ad}_{g} \xi \in \mathfrak{n}$ and we get

$$
A_{N}\left(g v_{q}\right)=\operatorname{Ad}_{g} \xi=\operatorname{Ad}_{g} A_{N}\left(v_{q}\right) \text { for all } q \in Q .
$$

Lemma 6.3.6. (a) Let $A_{N}$ be a connection on the principal bundle $Q$ with structure group $N$ having the property that for any $g \in G$ and any $v_{q} \in T_{q} Q$ we have $A_{N}\left(g v_{q}\right)=\operatorname{Ad}_{g} A_{N}\left(v_{q}\right)$. Let $\tilde{\nabla}^{A_{N}}$ be the covariant derivative on $\tilde{\mathfrak{n}}$ associated to $A_{N}$ according to Definition 2.3.1. Then $\tilde{\nabla}^{A_{N}}$ is $G / N$-invariant.

(b) Assume the same conditions as in (a). Then the action of $G / N$ on $T(Q / N) \oplus \tilde{\mathfrak{n}}$ defined by the natural actions of $G / N$ on $T(Q / N)$ and on $\tilde{\mathfrak{n}}$ commutes with the isomorphism $\alpha_{A_{N}}: T Q / N \rightarrow T(Q / N) \oplus \tilde{\mathfrak{n}}$ (see Lemma 2.4.2).

Proof. (a) Let $\tilde{D}^{A_{N}} / D t$ be the covariant derivative along curves associated to the connection $\tilde{\nabla}^{A_{N}}$. Using the previous lemmas, the proof of this part is straightforward. Indeed, according to Lemma 2.3.4 we have

$$
\frac{\tilde{D}^{A_{N}}[q(s), \xi(s)]_{G}}{D s}=\left[q(s),-\left[A_{N}(q(s), \dot{q}(s)), \xi(s)\right]+\dot{\xi}(s)\right]_{N} .
$$

Given any $[g]_{N} \in G / N$ we have

$$
\begin{aligned}
\frac{\tilde{D}^{A_{N}}\left[g q(s), \operatorname{Ad}_{g} \xi(s)\right]_{G}}{D s} & =\left[g q(s),-\left[A_{N}(g q(s), g \dot{q}(s)), \operatorname{Ad}_{g} \xi(s)\right]+\operatorname{Ad}_{g} \dot{\xi}(s)\right]_{N} \\
& =\left[g q(s),-\operatorname{Ad}_{g}\left[A_{N}(q(s), \dot{q}(s)), \xi(s)\right]+\operatorname{Ad}_{g} \dot{\xi}(s)\right]_{N} \\
& =[g]_{N}\left[q(s),-\left[A_{N}(q(s), \dot{q}(s)), \xi(s)\right]+\dot{\xi}(s)\right]_{N},
\end{aligned}
$$

which proves the $G / N$-invariance of the covariant derivative $\tilde{D}^{A_{N}} / D t$.

(b) This follows easily from the previous lemmas.

Theorem 6.3.7. Let $Q$ be a principal bundle with structure group $G$ and let $N$ be a normal subgroup of $G$. Let $A_{N}$ be a connection on the principal bundle $Q$ with structure group $N$ having the property that for any $g \in G$ and any $v_{q} \in T_{q} Q$ we have $A_{N}\left(g v_{q}\right)=\operatorname{Ad}_{g} A_{N}\left(v_{q}\right)$. Consider the action of $G / N$ on $T(Q / N) \oplus \tilde{\mathfrak{n}}$ such that 
its restriction to $\tilde{\mathfrak{n}}$ coincides with the natural action and its restriction to $T(Q / N)$ coincides with the tangent lift of the action of $G / N$ on $Q / N$ defined in Lemma 6.3.1. Then this action is an action in the category $\mathfrak{L P}$. The reduced bundle of $T(Q / N) \oplus \tilde{\mathfrak{n}}$ by the group $G / N$ with respect to any connection $A_{G / N}$ on the bundle $Q / N$ with structure group $G / N$ is an object of $\mathfrak{L P}$.

Proof. The proof is a direct consequence of Definition 6.2.4, Lemmas 6.3.4 and 6.3.6, and Theorem 6.2.10.

\section{More Preparatory Lemmas.}

Before we can state and prove one of our main results on Lagrangian reduction by stages we need a few more lemmas.

Lemma 6.3.8. Assume the conditions of Theorem 6.3.7 and, in addition, choose a connection $A_{G}$ on the principal bundle $Q$ with structure group $G$. Let $K=G / N$. Consider the following vector bundle isomorphisms:

(a) the quotient isomorphism (see Lemma 6.3.6 and Definition 6.2.11)

$$
\left[\alpha_{A_{N}}^{T Q}\right]_{G / N}:(T Q / N) /(G / N) \rightarrow(T(Q / N) \oplus \tilde{\mathfrak{n}}) /(G / N),
$$

(b) the isomorphism (see Lemma 6.3.1)

$$
i_{G / N}^{T Q}: T Q / G \rightarrow(T Q / N) /(G / N)
$$

(c) the isomorphism (see Definition 6.2.11)

$$
\alpha_{A_{G}}^{T Q}: T Q / G \rightarrow T(Q / G) \oplus \tilde{\mathfrak{g}},
$$

(d) the isomorphism (see Definition 6.2.11)

$$
\begin{aligned}
& \alpha_{A_{G / N}}^{T(Q / N) \oplus \tilde{\mathfrak{n}}}:(T(Q / N) \oplus \tilde{\mathfrak{n}}) /(G / N) \\
& \quad \rightarrow T((Q / N) /(G / N)) \oplus \tilde{\mathfrak{k}} \oplus(\tilde{\mathfrak{n}} /(G / N)),
\end{aligned}
$$

where $\mathfrak{k}$ is the Lie algebra of $K$ (and hence one has a Lie algebra isomorphism $\mathfrak{k} \equiv \mathfrak{g} / \mathfrak{n})$.

Then the linear isomorphisms, given by the push forward of maps corresponding to the previous vector bundle isomorphisms

$\left(a^{\prime}\right)$

$$
\left[\alpha_{A_{N}}^{T Q}\right]_{G / N *}: \Gamma((T Q / N) /(G / N)) \rightarrow \Gamma((T(Q / N) \oplus \tilde{\mathfrak{n}}) /(G / N))
$$

$$
i_{G / N *}^{T Q}: \Gamma(T Q / G) \rightarrow \Gamma((T Q / N) /(G / N))
$$


$\left(c^{\prime}\right)$

$$
\alpha_{A_{G^{*}}}^{T Q}: \Gamma(T Q / G) \rightarrow(T(Q / G) \oplus \tilde{\mathfrak{g}})
$$

$\left(d^{\prime}\right)$

$$
\begin{aligned}
& \alpha_{A_{G / N^{*}}^{T}}^{T(Q / N) \oplus \tilde{\mathfrak{n}}}: \Gamma((T(Q / N) \oplus \tilde{\mathfrak{n}}) /(G / N)) \\
& \quad \rightarrow \Gamma(T((Q / N) /(G / N)) \oplus \tilde{\mathfrak{k}} \oplus(\tilde{\mathfrak{n}} /(G / N)))
\end{aligned}
$$

are Lie algebra isomorphisms.

Consider the composition

(e)

$$
\epsilon_{\left(A_{N}, A_{G / N}, A_{G}\right)}^{T Q}=\alpha_{A_{G / N}}^{T(Q / N) \oplus \tilde{\mathfrak{n}}} \circ\left[\alpha_{A_{N}}^{T Q}\right]_{G / N} \circ i_{G / N}^{T Q} \circ\left(\alpha_{A_{G}}^{T Q}\right)^{-1},
$$

which is a vector bundle isomorphism from

$$
T(Q / G) \oplus \tilde{\mathfrak{g}} \quad \text { onto } \quad T((Q / N) /(G / N)) \oplus \tilde{\mathfrak{k}} \oplus(\tilde{\mathfrak{n}} /(G / N)) .
$$

Then $\epsilon_{\left(A_{N}, A_{G / N}, A_{G}\right)}^{T Q}$ induces a Lie algebra isomorphism

$\left(e^{\prime}\right)$

$$
\begin{aligned}
& \epsilon_{\left(A_{N}, A_{G / N}, A_{G}\right) *}^{T Q}: \Gamma((T(Q / G) \oplus \tilde{\mathfrak{g}})) \\
& \quad \rightarrow \Gamma(T((Q / N) /(G / N)) \oplus \tilde{\mathfrak{k}} \oplus(\tilde{\mathfrak{n}} /(G / N)))
\end{aligned}
$$

Proof. The conclusion follows using Lemmas 5.2.1, 6.3.6, 6.2.12, the paragraph on quotient Lie algebras in section $\S 5.2$, and standard results on quotient manifolds.

It is not generally true that $\epsilon_{\left(A_{N}, A_{G / N}, A_{G}\right)}^{T Q}$ is an isomorphism in the category $\mathfrak{L P}$, for arbitrary choices of the connections $A_{N}, A_{G / N}$, and $A_{G}$. Next we will show that it is always possible to choose $A_{N}, A_{G / N}$, and $A_{G}$ in such a way that $\epsilon_{\left(A_{N}, A_{G / N}, A_{G}\right)}^{T Q}$ is an isomorphism in the category $\mathfrak{L P}$.

Lemma 6.3.9. Assume the hypothesis of Lemma 6.3.5 and define the connection $A_{N}$ as in that lemma. Define the connection $A_{G}$ on the principal bundle $Q$ with structure group $G$ by the condition that, for all $q \in Q, \operatorname{Hor}_{q}^{A_{G}}(T Q)$ is the orthogonal complement of $\operatorname{Ver}_{q}^{G}(T Q)$. For each $[q]_{N} \in Q / N$ let

$$
H_{[q]_{N}}(T(Q / N))=T \pi_{N}\left(\operatorname{Hor}_{q}^{A_{G}}(T Q)\right),
$$

where $\pi_{N}: Q \rightarrow Q / N$ is the natural principal bundle projection. Then the collection of all $H_{[q]_{N}}(T(Q / N))$ as $[q]_{N}$ varies in $Q / N$ defines a connection $A_{G / N}$ on 
the principal bundle $Q / N$ with structure group $G / N$, by choosing these to be the horizontal spaces, that is,

$$
\operatorname{Hor}_{[q]_{N}}^{A_{G / N}}\left((T(Q / N)) \equiv H_{[q]_{N}}(T(Q / N)) .\right.
$$

The following property is satisfied: for all $v_{q} \in T Q$ and all $q \in Q$ we have $A_{G}\left(v_{q}\right)=0$ if and only if $A_{N}\left(v_{q}\right)=0$ and $A_{G / N}\left(T \pi_{N}\left(v_{q}\right)\right)=0$.

We shall omit the proof of this lemma which can be carried out in a standard way using the preceding results.

The next theorem, together with its generalization for arbitrary objects of $\mathfrak{L P}$ given in Theorem 6.3.14 constitutes one of our main results on Lagrangian reduction by stages.

Theorem 6.3.10. Assume the hypothesis of Theorem 6.3 .7 and, in addition, choose the connections $A_{N}, A_{G / N}$, and $A_{G}$ in such a way that the following property is satisfied: for all $v_{q} \in T Q$ and all $q \in Q$ we have $A_{G}\left(v_{q}\right)=0$ if and only if $A_{N}\left(v_{q}\right)=0$ and $A_{G / N}\left(T \pi_{N}\left(v_{q}\right)\right)=0$. Then $\epsilon_{\left(A_{N}, A_{G / N}, A_{G}\right)}^{T Q}$ (see Lemma 6.3.8) is an isomorphism in the category $\mathfrak{L P}$.

Proof. Taking into account Lemmas 6.3 .9 and 6.1 .3 we see that we only need to show that

$$
\epsilon_{\left(A_{N}, A_{G / N}, A_{G}\right)}^{T Q}(T(Q / G))=T((Q / N) /(G / N)) .
$$

However, this follows easily from the definition of $\epsilon_{\left(A_{N}, A_{G / N}, A_{G}\right)}^{T Q}$.

The following corollary gives a structure theorem for Lie algebras.

Corollary 6.3.11. Let $G$ be a Lie group, let $N$ be a normal subgroup, and let $K=G / N$. Let $\mathfrak{g}, \mathfrak{n}$, and $\mathfrak{k}$ be the Lie algebras of $G, N$, and $K$ respectively. Choose an identification $\mathfrak{g} \equiv \mathfrak{k} \oplus \mathfrak{n}$ as linear spaces. Choose any connection $A_{N}$ on the principal bundle $G$ with structure group $N$ such that $A_{N}\left(g v_{q}\right)=\operatorname{Ad}_{g} A_{N}\left(v_{q}\right)$ for all $g \in G$, all $v_{q} \in T_{q} G$, and all $q \in G$. Then the Lie algebra bracket on $\mathfrak{g}$ can be written in terms of the Lie algebra brackets on $\mathfrak{n}$ and $\mathfrak{k}$ and also $\nabla^{\left(A_{N}, V\right)}$ and $\tilde{B}^{A_{N}}$ as follows:

$$
\begin{gathered}
{\left[\kappa_{1} \oplus \eta_{1}, \kappa_{2} \oplus \eta_{2}\right]=\left[\kappa_{1}, \kappa_{2}\right] \oplus\left[\nabla^{\left(A_{N}, V\right)}\right]_{G / N, \kappa_{1}} \eta_{2}-\left[\nabla^{\left(A_{N}, V\right)}\right]_{G / N, \kappa_{2}} \eta_{1}} \\
-\left[\tilde{B}^{A_{N}}\right]_{G / N}\left(\kappa_{1}, \kappa_{2}\right)+\left[\eta_{1}, \eta_{2}\right] .
\end{gathered}
$$

Proof. The proof is a straightforward application of Theorems 6.2.10, 6.3.7, and 6.3.10.

Now we shall generalize Theorem 6.3.7, Lemma 6.3.8, and Theorem 6.3.10 for arbitrary objects $T Q \oplus V$ rather than the particular case $V=0$ considered in those results. We will give precise statements, but we shall omit the proofs since they are entirely similar to the proofs of Theorem 6.3.7, Lemma 6.3.8, and Theorem 6.3.10. 
Theorem 6.3.12. Let $W=T Q \oplus V$ be an object of $\mathfrak{L P}$ and let $G \times W \rightarrow W$ be an action in the category $\mathfrak{L P}$ of a Lie group $G$ on $W$; in particular, this action induces a principal bundle structure on $Q$ with structure group $G$. Let $N$ be a normal subgroup of $G$ and let $A_{N}$ be a connection on the principal bundle $Q$ with structure group $N$ having the property that for any $g \in G$ and any $v_{q} \in T_{q} Q$ we have $A_{N}\left(g v_{q}\right)=\operatorname{Ad}_{g} A_{N}\left(v_{q}\right)$. Consider the action of $G / N$ on $T(Q / N) \oplus \tilde{\mathfrak{n}} \oplus(V / N)$ such that its restriction to $T(Q / N) \oplus \tilde{\mathfrak{n}}$ coincides with the action defined in Theorem 6.3.7 and its restriction to $V / N$ is the quotient action of the action of $G$ on $V$ by $N$ (see Lemma 5.2.1). Then this is an action in the category $\mathfrak{L P}$. The quotient bundle of $T(Q / N) \oplus \tilde{\mathfrak{n}} \oplus(V / N)$ by the group $G / N$ with respect to any connection $A_{G / N}$ on the bundle $Q / N$ with structure group $G / N$ is an object of $\mathfrak{L P}$.

Lemma 6.3.13. Assume the conditions of Theorem 6.3 .12 and, in addition, choose a connection $A_{G}$ on the principal bundle $Q$ with structure group $G$. Let $K=G / N$. Consider the following vector bundle isomorphisms:

(a) the quotient isomorphism (see Lemma 6.3.6)

$$
\left[\alpha_{A_{N}}^{W}\right]_{G / N}:(W / N) /(G / N) \rightarrow(T(Q / N) \oplus \tilde{\mathfrak{n}} \oplus(V / N)) /(G / N),
$$

(b) the isomorphism (see Lemma 6.3.1)

$$
i_{G / N}^{W}: W / G \rightarrow(W / N) /(G / N),
$$

(c) the isomorphism (see Definition 6.2.11)

$$
\alpha_{A_{G}}^{W}: W / G \rightarrow T(Q / G) \oplus \tilde{\mathfrak{g}} \oplus(V / G),
$$

(d) the isomorphism (see Definition 6.2.11)

$$
\begin{aligned}
& \alpha_{A_{G / N}}^{T(Q / N) \oplus \tilde{\mathfrak{n}} \oplus(V / N)}:(T(Q / N) \oplus \tilde{\mathfrak{n}} \oplus(V / N)) /(G / N) \\
& \quad \rightarrow T((Q / N) /(G / N)) \oplus \tilde{\mathfrak{k}} \oplus(\tilde{\mathfrak{n}} \oplus(V / N)) /(G / N),
\end{aligned}
$$

where $\tilde{\mathfrak{k}}$ is the Lie algebra of $K$.

Then the corresponding linear isomorphisms

(a')

$$
\left[\alpha_{A_{N}}^{W}\right]_{G / N *}: \Gamma((W / N) /(G / N)) \rightarrow \Gamma((T(Q / N) \oplus \tilde{\mathfrak{n}} \oplus(V / N)) /(G / N))
$$

$$
i_{G / N *}^{W}: \Gamma(W / G) \rightarrow \Gamma((W / N) /(G / N))
$$

$\left(c^{\prime}\right)$

$$
\alpha_{A_{G} *}^{W}: \Gamma(W / G) \rightarrow \Gamma(T(Q / G) \oplus \tilde{\mathfrak{g}} \oplus(V / G))
$$


$\left(d^{\prime}\right)$

$$
\begin{aligned}
& \alpha_{A_{G / N} *}^{T(Q / N) \oplus \tilde{\mathfrak{n}} \oplus(V / N)}: \Gamma((T(Q / N) \oplus \tilde{\mathfrak{n}} \oplus(V / N)) /(G / N)) \\
& \quad \rightarrow \Gamma(T((Q / N) /(G / N)) \oplus \tilde{\mathfrak{k}} \oplus(\tilde{\mathfrak{n}} \oplus(V / N)) /(G / N))
\end{aligned}
$$

are Lie algebra isomorphisms. Consider the composition

(e)

$$
\epsilon_{\left(A_{N}, A_{G / N}, A_{G}\right)}^{W}=\alpha_{A_{G / N}}^{T(Q / N) \oplus \tilde{\mathfrak{n}} \oplus(V / N)} \circ\left[\alpha_{A_{N}}^{W}\right]_{G / N} \circ i_{G / N}^{W} \circ\left(\alpha_{A_{G}}^{W}\right)^{-1},
$$

which is a vector bundle isomorphism from $T(Q / G) \oplus \tilde{\mathfrak{g}} \oplus(V / G)$ onto

$$
T((Q / N) /(G / N)) \oplus \tilde{\mathfrak{k}} \oplus(\tilde{\mathfrak{n}} \oplus(V / N)) /(G / N) .
$$

Then $\epsilon_{\left(A_{N}, A_{G / N}, A_{G}\right)}^{W}$ induces a Lie algebra isomorphism

$\left(\mathrm{e}^{\prime}\right)$

$$
\begin{aligned}
& \epsilon_{\left(A_{N}, A_{G / N}, A_{G}\right) *}^{W}: \Gamma((T(Q / G) \oplus \tilde{\mathfrak{g}} \oplus(V / G)) \\
& \quad \rightarrow \Gamma(T((Q / N) /(G / N)) \oplus \tilde{\mathfrak{k}} \oplus \tilde{\mathfrak{n}} /(G / N) \oplus((V / N) /(G / N)))
\end{aligned}
$$

Theorem 6.3.14. Assume the hypotheses of Theorem 6.3.12 and, in addition, choose the connections $A_{N}, A_{G / N}$ and $A_{G}$ in such a way that the following property is satisfied: for all $v_{q} \in T Q$ and all $q \in Q$ we have $A_{G}\left(v_{q}\right)=0$ if and only if $A_{N}\left(v_{q}\right)=0$ and $A_{G / N}\left(T \pi_{N}\left(v_{q}\right)\right)=0$. Then $\epsilon_{\left(A_{N}, A_{G / N}, A_{G}\right)}^{W}$ is an isomorphism in the category $\mathfrak{L P}$.

\subsection{The Subcategory $\mathfrak{R T}$ and Reduction by Stages of Variational Principles on $T Q$.}

Let $\mathcal{T}$ be the category of tangent bundles of manifolds and tangent lifts of maps, which is a subcategory of $\mathfrak{L P}$. The category $\mathfrak{R T}$ is defined as the smallest subcategory of $\mathfrak{L P}$ that contains $\mathcal{T}$ and is closed under the quotienting operation. Thus, as vector bundles, elements of $\mathfrak{R T}$ are constructed by an inductive procedure of the type $V_{0}=T Q, V_{i+1}=V_{i} / G_{i}$, where for each $i=1,2, \ldots, G_{i}$ is a group acting on $V_{i}$. Using this and the fact that tangent bundles are always orientable manifolds we can easily prove that objects of $\mathfrak{R T}$ are orientable manifolds. This shows in particular that $\mathfrak{R T}$ does not coincide with $\mathfrak{L P}$ since there are simple examples of objects of $\mathfrak{L P}$ that are nonorientable manifolds. For instance, let $Q \equiv S^{1}$ and consider the vector bundle $T Q \oplus M$, where $M$ is the Möbius band; thus, as a manifold, $T Q \oplus M$ is nonorientable. The Lie algebra structure on the fibers of $M$ must be 0 because the fibers are 1-dimensional. The $M$-valued 2-form $\omega$ must be 0 because $Q$ is 1 dimensional. Now choose a vector bundle metric on $M$. Then define the connection 
$\nabla$ on the vector bundle $M$ by the condition that a curve in $M$ is horizontal if and only if its distance to the 0 section is constant. Then we can check that, with this structure, $T Q \oplus M$ is an object of $\mathfrak{L P}$.

It is immediate that all the results of the previous sections involving generic objects of $\mathfrak{L P}$ are valid for generic objects of $\mathfrak{R T}$. A study of the local structure of objects of $\mathfrak{R T}$ or $\mathfrak{L P}$ and the study of categories bigger that $\mathfrak{L P}$, which appear in the study of nonholonomic systems, Poisson geometry, and other topics is being planned for future work. See Cendra, Marsden and Ratiu (2000) for work in this direction for nonholonomic systems. In this section, we shall present the theory of Lagrangian reduction by stages for the bundle $T Q$, where $Q$ is a principal bundle with structure group $G$ having a normal subgroup $N$. This theory explains how Hamilton's variational principle corresponding to a given $G$-invariant, and hence $N$-invariant, Lagrangian $L: T Q \rightarrow \mathbb{R}$ is reduced by stages, first under the action of $N$ and then under the action of $G / N$. The reduced bundles for these reduced variational principles and corresponding Lagrange-Poincaré operators are elements of $\mathfrak{R T}$, as we saw in section $\S 3.2$, where the case of the first stage reduction was studied. A more general theory of reduction by stages of variational principles for any given object of $\mathfrak{L P}$ including the reduction of the corresponding variational principles is being planned for future work.

We shall begin with some remarks on the geometry of variations, using a notation slightly more precise than the one used in sections $\S 2.1, \S 3.1$, and $\S 3.2$. In this section, we will often use, for short, the notation $T Q \oplus V$ to denote an element of $\mathfrak{R T}$, meaning that this bundle is isomorphic to a bundle of the type $T(M / K) \oplus \tilde{\mathfrak{k}}$, where $M$ is a principal bundle with structure group $G$.

Let $Q$ be a manifold. The tangent lift of a given curve $q \in \Omega(Q)$ is the curve $\ell(q)$ in $q \in \Omega(T Q)$ defined by $\ell(q)(t)=(q(t), \dot{q}(t))$ for all $t$; thus we obtain a map $\ell: \Omega(Q) \rightarrow \Omega(T Q)$.

The space of allowed variations of a curve $q$ is the tangent space $\Delta_{q} \equiv$ $T_{q} \Omega\left(Q ; q_{0}, q_{1}\right)$. Thus, elements of $\Delta_{q}$ are variations $\delta q$ such that $\delta q\left(t_{i}\right)=0$ for $i=1,2$, where, as usual, $\delta q$ is the derivative of some deformation $q_{\lambda}(t)$, that is, for all $t$, we have

$$
\delta q(t)=\left.\frac{d}{d \lambda} q_{\lambda}(t)\right|_{\lambda=0} .
$$

We have a canonical inclusion map $T_{q} \Omega(Q) \rightarrow \Omega(T Q)$. The space of lifted allowed variations of a curve $\ell(q)$ is the subspace $\Delta_{q}^{\ell}$ of $T_{\ell(q)} \Omega(T Q)$ defined by

$$
\Delta_{q}^{\ell}=T_{q} \ell\left(T_{q} \Omega\left(Q ; q_{0}, q_{1}\right)\right)
$$

where

$$
T_{q} \ell: T_{q} \Omega(Q) \rightarrow T_{\ell(q)} \Omega(T Q)
$$

is the tangent map of $\ell$. A generic element of $\Delta_{q}^{\ell}$ can therefore be written $T_{q} \ell \cdot \delta q$, for some variation $\delta q$ of $q$ such that $\delta q\left(t_{i}\right)=0$ for $i=1,2$. It is easy to prove that the restriction of $T_{q} \ell$ to $T_{q} \Omega\left(Q ; q_{0}, q_{1}\right) \equiv \Delta_{q}$ is a linear bijective map onto $\Delta_{q}^{\ell}$.

The following definition is inspired by Lemmas 3.1.4 and 3.1.7. 
Definition 6.4.1. Let $T Q \oplus V$ be an object of $\mathfrak{R T}$ and let $\tau: V \rightarrow Q$ be the projection. We denote by $\ell \Omega(Q) \oplus \Omega(V)$ the set of all $\ell(q) \oplus v \in \Omega(T Q \oplus V)$. Likewise $\ell \Omega\left(Q ; q_{0}\right) \oplus \Omega\left(V ; q_{0}\right)$ is the subset of all $\ell(q) \oplus v \in \Omega(T Q \oplus V)$ such that $q\left(t_{0}\right)=q_{0}$ and $\ell \Omega\left(Q ; q_{0}, q_{1}\right) \oplus \Omega\left(V ; q_{0}, q_{1}\right)$ is the subset of all $\ell(q) \oplus v \in \Omega(T Q \oplus V)$ such that $q\left(t_{0}\right)=q_{0}$ and $q\left(t_{1}\right)=q_{1}$. Let $v$ be a curve in $V$ and let $q=\tau v$. We will sometimes think of the manifold $Q$ as being identified with the vector bundle having base $Q$ and 0 -dimensional fiber. Thus if $q=\tau v$ we can write $v \equiv q \oplus v$ (see section §2.1) The space of allowed variations of $q \oplus v$ is the subspace $\Delta_{q \oplus v}$ of $T_{q} \Omega(Q) \oplus \Omega(V)$ of all curves of the type

$$
\delta q \oplus \delta v=\delta q \oplus \frac{D w}{D t}+[v, w]-\omega(q)(\dot{q}, \delta q),
$$

where $\delta q$ is a variation of $q$ with $\delta q\left(t_{i}\right)=0$ for $i=1,2$, that $i s, \delta q \in \Delta_{q}$, and $w$ is a curve in $V$ such that $\tau(w(t))=q(t)$ for all $t$ and $w\left(t_{i}\right)=0$ for $i=1,2$. The space of lifted allowed variations of a curve $\ell(q) \oplus v \in \Omega(T Q \oplus V)$ is the subspace

$$
\Delta_{q \oplus v}^{\ell}:=\Delta_{q}^{\ell} \oplus \Omega(V) \quad \text { of } \quad T_{\ell(q)} \Omega(T Q) \oplus \Omega(V)
$$

of all variations of the type

$$
T_{q} \ell \cdot \delta q \oplus \frac{D w}{D t}+[v, w]-\omega(q)(\dot{q}, \delta q),
$$

where $\delta q$ is a variation of $q$ with $\delta q\left(t_{i}\right)=0$ for $i=1,2, w$ is a curve in $V$ such that $\tau(w(t))=q(t)$ for all $t$, and $w\left(t_{i}\right)=0$ for $i=1,2$.

Assume that the group $G$ acts on the manifold $Q$. Then we have maps (see Lemma 5.2.1 and Lemma 2.4.2)

$$
\pi_{G}(T Q): T Q \rightarrow T Q / G \quad \text { and } \quad \alpha_{A}: T Q / G \rightarrow T(Q / G) \oplus \tilde{\mathfrak{g}} .
$$

Let $\ell(q) \in \Omega(T Q)$. Then we have

$$
\Omega\left(\alpha_{A} \circ \pi_{G}(T Q)\right)(\ell(q))=\ell(x) \oplus \bar{\xi}
$$

where

$$
x(t)=[q]_{G}(t) \quad \text { and } \quad \bar{\xi}(t)=[q(t), A(q(t), \dot{q}(t))]_{G}
$$

for all $t$.

Lemma 6.4.2. Assume the hypothesis of Lemma 2.4.2. Then the map

$$
\Omega\left(\alpha_{A} \circ \pi_{G}(T Q)\right): \Omega(T Q) \rightarrow \Omega(T(Q / G) \oplus \tilde{\mathfrak{g}})
$$

restricted to $\ell \Omega\left(Q ; q_{0}\right)$ is injective. The image of this restriction is

$$
\ell \Omega\left(Q / G ; x_{0}\right) \oplus \Omega\left(\tilde{\mathfrak{g}} ; x_{0}\right) .
$$


Proof. Let $\dot{x}(t) \oplus \bar{\xi}(t)=\ell(x)(t) \oplus \bar{\xi}(t)$ be given such that $x\left(t_{0}\right)=x_{0}$. We must show that there is a unique curve $q \in \Omega\left(Q ; q_{0}\right)$ such that

$$
\Omega\left(\alpha_{A} \circ \pi_{G}(T Q)\right) \ell(q)=\ell(x) \oplus \bar{\xi} .
$$

We can always write $\bar{\xi}(t)=\left[x_{q_{0}}^{h}(t), \xi(t)\right]_{G}$. Let $q(t)=g(t) x_{q_{0}}^{h}(t)$ where $g(t)$ is a curve in $G$. Then we can see that

$$
\Omega\left(\alpha_{A} \circ \pi_{G}(T Q)\right) \ell(q)=\left(\dot{x}(t) \oplus\left[x_{q_{0}}^{h}(t), \dot{g}(t) g^{-1}(t)\right]_{G}\right) .
$$

Thus, $g(t)$ must satisfy $\dot{g}(t)=\xi(t) g(t)$ for all $t$ and $g\left(t_{0}\right)=e$.

Corollary 6.4.3. The map

$$
\Omega\left(\alpha_{A} \circ \pi_{G}(T Q)\right): \Omega(T Q) \rightarrow \Omega(T(Q / G) \oplus \tilde{\mathfrak{g}})
$$

restricted to $\ell \Omega\left(Q ; q_{0}, q_{1}\right)$ is injective.

Lemma 6.4.4. Assume the hypothesis of Lemma 6.4.2. Then the restriction of the map $T_{q} \Omega\left(\alpha_{A} \circ \pi_{G}(T Q)\right)$ to $\Delta_{q}^{\ell}$ is a linear isomorphism onto $\Delta_{x \oplus \bar{\xi}}^{\ell}$, where

$$
\dot{x} \oplus \bar{\xi}=\ell(x) \oplus \bar{\xi}=\Omega\left(\alpha_{A} \circ \pi_{G}(T Q)\right) \ell(q)
$$

and $\Delta_{x \oplus \bar{\xi}}^{\ell}$ is the space of lifted allowed variations of $\ell(x) \oplus \bar{\xi}$ (see Definition 6.4.1).

Proof. Using Lemmas 3.1.4, 3.1.7, and 6.4 .2 and considering that an element of $\Delta_{q}^{\ell}$ can be represented as the derivative of a deformation $\ell\left(\left(q_{\lambda}(t)\right)\right.$ of a curve $q(t) \equiv q_{0}(t)$ at $\lambda=0$, it follows easily that

$$
T_{q} \Omega\left(\alpha_{A} \circ \pi_{G}(T Q)\right) \Delta_{q}^{\ell} \subset \Delta_{x \oplus \bar{\xi}}^{\ell} .
$$

Bijectiveness of the restriction $T_{q} \Omega\left(\alpha_{A} \circ \pi_{G}(T Q)\right) \mid \Delta_{q}^{\ell}$ can be proved using a reconstruction procedure as in the proof of Lemma 6.4.2. Namely, let $\ell\left(x_{\lambda}\right) \oplus \bar{\xi}_{\lambda}$ be given a deformation of $\ell(x) \oplus \bar{\xi}$, where $\bar{\xi}_{\lambda}(t)=\left[x_{q_{0} \lambda}^{h}(t), \xi_{\lambda}(t)\right]_{G}$. This gives a unique deformation $g_{\lambda}(t)$ of $g(t)$ satisfying $\dot{g}_{\lambda}(t)=\xi_{\lambda}(t) g_{\lambda}(t)$, which in turn gives a unique deformation $q_{\lambda}(t)=g_{\lambda}(t) x_{q_{0} \lambda}^{h}(t)$ of $q(t)$. Therefore by differentiation with respect to $\lambda$ at $\lambda=0$ we obtain the inverse of the tangent map $T_{q} \Omega\left(\alpha_{A} \circ \pi_{G}(T Q)\right)$.

Lemma 6.4.2 and Corollary 6.4.3 can be easily generalized for any object of $\mathfrak{L P}$ instead of $T Q$. However, a generalization of Lemma 6.4.4 involves a careful study of the geometry of the space of variations and will be the purpose of future work on general Lagrangian reduction in the category $\mathfrak{L P}$. More precisely, we have

Lemma 6.4.5. Assume the hypothesis of Lemma 6.4.2 and, in addition, let $W=$ $T Q \oplus V$ be an element of $\mathfrak{L P}$. Then the map

$$
\Omega\left(\alpha_{A}^{W} \circ \pi_{G}(W)\right): \Omega(W) \rightarrow \Omega(T(Q / G) \oplus \tilde{\mathfrak{g}} \oplus(V / G))
$$

restricted to $\ell \Omega\left(Q ; q_{0}\right) \oplus \Omega\left(V ; q_{0}\right)$ is injective. The image of this restriction is

$$
\ell \Omega\left(Q / G ; x_{0}\right) \oplus \Omega\left(\tilde{\mathfrak{g}} ; x_{0}\right) \oplus \Omega\left(V / G ; x_{0}\right) .
$$


Proof. For a given curve

$$
\dot{x}(t) \oplus \bar{\xi}(t) \oplus[v]_{G}(t) \quad \text { with } \quad x\left(t_{0}\right)=x_{0}
$$

we find, using the curve $\dot{x}(t) \oplus \bar{\xi}(t)$, the curve $q \in \Omega\left(Q ; q_{0}\right)$ as in Lemma 6.4.2. Then using Lemma 5.2.1 we see that there is a unique curve $v \in \Omega(V)$ such that $\tau v(t)=q(t)$, where $\tau: V \rightarrow Q$ is the projection of $V$, and its class is $[v]_{G}(t)$ for all $t$.

We now state the generalization of Corollary 6.4.3.

Corollary 6.4.6. The map

$$
\Omega\left(\alpha_{A}^{W} \circ \pi_{G}(W)\right): \Omega(W) \rightarrow \Omega(T(Q / G) \oplus \tilde{\mathfrak{g}} \oplus(V / G))
$$

restricted to $\ell\left(\Omega\left(Q ; q_{0}, q_{1}\right)\right) \oplus \Omega(V)$ is injective.

Next we will see how the space of lifted allowed variations is transformed under reduction by stages.

Lemma 6.4.7. Assume the hypothesis of Theorem 6.3.14. Then we have

$$
\begin{aligned}
& \epsilon_{\left(A_{N}, A_{G / N}, A_{G}\right)}^{W} \circ \alpha_{A_{G}}^{W} \circ \pi_{G}(W) \\
& =\alpha_{A_{G / N}^{T}}^{T(Q / N) \oplus \tilde{\mathfrak{n}} \oplus(V / N)} \circ \pi_{G / N}(T(Q / N) \oplus \tilde{\mathfrak{n}} \oplus(V / N)) \circ \alpha_{A_{N}}^{W} \circ \pi_{N}(W) .
\end{aligned}
$$

Proof. We can prove in a straightforward manner that

$$
\begin{aligned}
i_{G / N}^{W} \circ \pi_{G}(W) & =\pi_{G / N}(W / N) \circ \pi_{N}(W) \\
{\left[\alpha_{A_{N}}^{W}\right]_{G / N} \circ \pi_{G / N}(W / N) } & =\pi_{G / N}(T(Q / N) \oplus \tilde{\mathfrak{n}} \oplus(V / N)) \circ \alpha_{A_{N}}^{W} .
\end{aligned}
$$

Using this and the definition of $\epsilon_{\left(A_{N}, A_{G / N}, A_{G}\right)}^{W}$ the lemma follows.

Theorem 6.4.8. Assume the hypothesis of Theorem 6.3.10. Then we have

$$
\begin{aligned}
& T \Omega\left(\epsilon_{\left(A_{N}, A_{G / N}, A_{G}\right)}^{T Q}\right) \circ T \Omega\left(\alpha_{A_{G}}^{T Q} \circ \pi_{G}(T Q)\right) \\
& =T \Omega\left(\alpha_{A_{G / N}^{T}}^{T(Q / N) \oplus \tilde{\mathfrak{n}}} \circ \pi_{G / N}(T(Q / N) \oplus \tilde{\mathfrak{n}})\right) \circ T \Omega\left(\alpha_{A_{N}}^{T Q} \circ \pi_{N}(T Q)\right) .
\end{aligned}
$$

Let $q \in \Omega(Q)$ and let

$$
\begin{aligned}
& \Omega\left(\alpha_{A_{G}}^{T Q} \circ \pi_{G}(T Q)\right)(\ell(q))=[q]_{G} \oplus \bar{\xi}, \\
& \Omega\left(\alpha_{A_{N}}^{T Q} \circ \pi_{N}(T Q)\right)(\ell(q))=[q]_{N} \oplus \bar{\eta},
\end{aligned}
$$

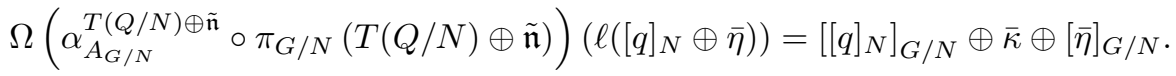


Then

$$
\begin{aligned}
& T \Omega\left(\alpha_{A_{G}}^{T Q} \circ \pi_{G}(T Q)\right): \Delta_{q}^{\ell} \rightarrow \Delta_{[q]_{G} \oplus \bar{\xi}}^{\ell}, \\
& T \Omega\left(\alpha_{A_{N}}^{T Q} \circ \pi_{N}(T Q)\right): \Delta_{q}^{\ell} \rightarrow \Delta_{[q]_{N} \oplus \bar{\eta}}^{\ell}, \\
& T \Omega\left(\alpha_{A_{G / N}^{T}}^{T(Q / N) \oplus \tilde{\mathfrak{n}}} \circ \pi_{G / N}(T(Q / N) \oplus \tilde{\mathfrak{n}})\right): \Delta_{[q]_{N} \oplus \bar{\eta}}^{\ell} \rightarrow \Delta_{\left[[q]_{N}\right]_{G / N} \oplus \bar{\kappa} \oplus[\bar{\eta}]_{G / N}}^{\ell}, \\
& T \Omega\left(\epsilon_{\left(A_{N}, A_{G / N}, A_{G}\right)}^{T Q}\right): \Delta_{[q]_{G} \oplus \bar{\xi}}^{\ell} \rightarrow \Delta_{\left[[q]_{N}\right]_{G / N} \oplus \bar{\kappa} \oplus[\bar{\eta}]_{G / N}}^{\ell}
\end{aligned}
$$

are linear isomorphisms.

Proof. The first equality in the statement of the theorem is a consequence of Lemma 6.4 .7 for $V=0$. The fact that

$$
\begin{aligned}
& T \Omega\left(\alpha_{A_{G}}^{T Q} \circ \pi_{G}(T Q)\right): \Delta_{q}^{\ell} \rightarrow \Delta_{[q]_{G} \oplus \bar{\xi}}^{\ell} \\
& T \Omega\left(\alpha_{A_{N}}^{T Q} \circ \pi_{N}(T Q)\right): \Delta_{q}^{\ell} \rightarrow \Delta_{[q]_{N} \oplus \bar{\eta}}^{\ell}, \\
& T \Omega\left(\epsilon_{\left(A_{N}, A_{G / N}, A_{G}\right)}^{T Q}\right): \Delta_{[q]_{G} \oplus \bar{\xi}}^{\ell} \rightarrow \Delta_{\left[[q]_{N}\right]_{G / N} \oplus \bar{\kappa} \oplus[\bar{\eta}]_{G / N}}^{\ell}
\end{aligned}
$$

are linear isomorphisms is a consequence of Lemma 6.4.4 and Theorem 6.3.10. Then it follows from the first equality in the statement of the theorem that also

$$
T \Omega\left(\alpha_{A_{G / N}}^{T(Q / N) \oplus \tilde{\mathfrak{n}}} \circ \pi_{G / N}(T(Q / N) \oplus \tilde{\mathfrak{n}})\right): \Delta_{[q]_{N} \oplus \bar{\eta}}^{\ell} \rightarrow \Delta_{\left[[q]_{N}\right]_{G / N} \oplus \bar{\kappa} \oplus[\bar{\eta}]_{G / N}}^{\ell}
$$

is a linear isomorphism.

The previous theorem says that, in the process of reducing the bundle $T Q$ by stages, the spaces of lifted allowed variations can also be reduced by stages. This leads to reducing variational principles by stages, as we will see next.

First we need to generalize the notion of action defined by a Lagrangian. Let $T Q \oplus V$ be an object of $\mathfrak{L P}$, where $\tau: V \rightarrow Q$ is the projection of $V$, and the structure on $V$ is given by $\nabla, \omega,[$,$] . Let$

$$
L: T Q \oplus V \rightarrow \mathbb{R}
$$

be a given Lagrangian, let $v \in \Omega(V)$ be a given curve, and let $q=\tau v$. The action of $L$ at the curve $v \in \Omega(V)$ is, by definition,

$$
J(L)(q)=\int_{t_{0}}^{t_{1}} L(q, \dot{q}, v) d t .
$$

We will also call this quantity the action of $L$ at the curve $\ell(q) \oplus v$ of $\Omega(T Q \oplus V)$ and denote it also by $J(L)(\ell(q))$.

The following definitions are obviously inspired by the results of $\S 3.2$. The LagrangePoincaré operator is a bundle map

$$
\mathcal{L P}(L): T^{(2)} Q \times_{Q / G} 2 V \rightarrow T^{*} Q \oplus V^{*}
$$


defined by

$$
(\mathcal{L P})(L)(\delta q \oplus \delta v)=\operatorname{Hor}(\mathcal{L P})(L) \delta q+\operatorname{Ver}(\mathcal{L P})(L) \delta v,
$$

where the vertical and horizontal Lagrange-Poincaré operators are given by

$$
\begin{aligned}
& \operatorname{Ver}(\mathcal{L P})(L) \delta v=\left(-\frac{D}{D t} \frac{\partial L}{\partial v}(q, \dot{q}, v)+\operatorname{ad}_{v}^{*} \frac{\partial L}{\partial v}(q, \dot{q}, v)\right) \delta v \\
& \operatorname{Hor}(\mathcal{L P})(L) \delta q=\left(\frac{\partial L}{\partial q}(q, \dot{q}, v)-\frac{D}{D t} \frac{\partial L}{\partial \dot{q}}(q, \dot{q}, v)\right) \delta q-\frac{\partial L}{\partial v}(q, \dot{q}, v) \omega(q)(\dot{q}, \delta q) .
\end{aligned}
$$

Here we must choose an arbitrary affine connection $\nabla$ on $Q$ to make sense of the covariant derivatives, in a similar way as we explained in $\S 3.2$ for the case $V=\tilde{\mathfrak{g}}$. For instance, in local coordinates, we can choose the Euclidean connection.

Now assume that there is an action of the group $G$ on $T Q \oplus V$ in the category $\mathfrak{L P}$ and that the Lagrangian $L$ is invariant under the action. Then for any choice of a connection $A_{G}$ on the principal bundle $Q$ we can identify

$$
(T Q \oplus V) / G \quad \text { with } \quad T(Q / G) \oplus \tilde{\mathfrak{g}} \oplus(V / G)
$$

via the isomorphism $\alpha_{A_{G}}^{T Q \oplus V}$ and we obtain an induced Lagrangian

$$
L^{(G)}: T(Q / G) \oplus \tilde{\mathfrak{g}} \oplus(V / G) \rightarrow \mathbb{R}
$$

called the reduced Lagrangian (to make the notation consistent with the one used in the particular case $V=0$ considered in Theorem 3.3.4 we should write $l \equiv L^{(G)}$, in that particular case). If, furthermore, $L^{(G)}$ is invariant under the action of another group $K$ on the object

$$
T(Q / G) \oplus \tilde{\mathfrak{g}} \oplus(V / G)
$$

in the category $\mathfrak{L P}$, and we choose a connection $A_{K}$ on the principal bundle $Q / G$ with structure group $K$, then we will denote the reduced Lagrangian simply by $L^{(G, K)}$ instead of $\left(L^{(G)}\right)^{(K)}$. We have

$$
\left.L^{(G, K)}: T((Q / G) / K) \oplus \tilde{\mathfrak{k}} \oplus(\tilde{\mathfrak{g}} / K) \oplus(V / G) / K\right) \rightarrow \mathbb{R} .
$$

As we said before, in this work we will explain how to perform reduction by stages only for the case $V=0$, leaving a more general theory for future work.

Assume the hypothesis of Theorem 6.4.8, let $K=G / N$, and let $L: T Q \rightarrow \mathbb{R}$ be a given Lagrangian. Then we have reduced Lagrangians

$$
\begin{aligned}
& L^{(G)}: T(Q / G) \oplus \tilde{\mathfrak{g}} \rightarrow \mathbb{R}, \\
& L^{(N)}: T(Q / N) \oplus \tilde{\mathfrak{n}} \rightarrow \mathbb{R}
\end{aligned}
$$

and

$$
L^{(N, K)}: T((Q / N) / K) \oplus \tilde{\mathfrak{k}} \oplus(\tilde{\mathfrak{n}} / K) \rightarrow \mathbb{R}
$$


For any given curve $q \in \Omega(Q)$ let

$$
\begin{aligned}
& \Omega\left(\alpha_{A_{G}}^{T Q} \circ \pi_{G}(T Q)\right)(\ell(q))=x \oplus \bar{\xi}, \\
& \Omega\left(\alpha_{A_{N}}^{T Q} \circ \pi_{N}(T Q)\right)(\ell(q))=y \oplus \bar{\eta}, \\
& \Omega\left(\alpha_{A_{K}}^{T(Q / N) \oplus \tilde{\mathfrak{n}}} \circ \pi_{K}(T(Q / N) \oplus \tilde{\mathfrak{n}})\right)(\ell(y \oplus \bar{\eta}))=z \oplus \bar{\kappa} \oplus[\bar{\eta}]_{K},
\end{aligned}
$$

as in Theorem 6.4.8. The next theorem is the main result of this section.

Theorem 6.4.9. Assume the situation explained above. Then the following conditions are equivalent:

(i) The curve $q(t)$ is a critical point of the action functional

$$
\int_{t_{0}}^{t_{1}} L(q, \dot{q}) d t
$$

with restrictions on the variations given by $\Delta_{q}^{\ell}$.

(ii) The Euler-Lagrange equations

$$
(\mathcal{E L})(L)(q)=0
$$

are satisfied.

(iii) The curve $x(t) \oplus \bar{\xi}(t)$ is a critical point of the action functional

$$
\int_{t_{0}}^{t_{1}} L^{(G)}(x(t), \dot{x}(t), \bar{\xi}(t)) d t
$$

with restrictions on the variations given by $\Delta_{x \oplus \bar{\xi}}^{\ell}$.

(iv) The Lagrange-Poincaré equations

$$
\mathcal{L} \mathcal{P}\left(L^{(G)}\right)(x \oplus \bar{\xi})=0
$$

are satisfied.

(v) The curve $y(t) \oplus \bar{\eta}(t)$ is a critical point of the action functional

$$
\int_{t_{0}}^{t_{1}} L^{(N)}(y(t), \dot{y}(t), \bar{\eta}(t)) d t
$$

with restrictions on the variations given by $\Delta_{y \oplus \bar{\eta}}^{\ell}$.

(vi) The Lagrange-Poincaré equations

$$
\mathcal{L P}\left(L^{(N)}\right)(y \oplus \bar{\eta})=0
$$

are satisfied. 
(vii) The curve $z \oplus \bar{\kappa} \oplus[\bar{\eta}]_{K}$ is a critical point of the action functional

$$
\int_{t_{0}}^{t_{1}} L^{(N, K)}\left(z \oplus \bar{\kappa} \oplus[\bar{\eta}]_{K}\right) d t
$$

with restrictions on the variations given by $\Delta_{z \oplus \bar{\kappa} \oplus[\bar{\eta}]_{K}}$.

(viii) The Lagrange-Poincaré equations

$$
\mathcal{L P}\left(L^{(N, K)}\right)\left(z \oplus \bar{\kappa} \oplus[\bar{\eta}]_{K}\right)=0
$$

are satisfied.

We will omit the proof of this theorem. It can be easily performed using an argument similar to the one used in the proof of Theorems 3.3.4 and 3.4.1 and also using Theorem 6.4.8.

\section{Remark.}

The main point of Theorem 6.4.9 is the equivalence with the last two statements (vii) and (viii), since the equivalence between the statements (i) to (vi) has been already established in Theorem 3.4.1. This shows that one can write the EulerLagrange equations for the Lagrangian

$$
L^{(N, K)}: T((Q / N) / K) \oplus \tilde{\mathfrak{k}} \oplus(\tilde{\mathfrak{n}} / K) \rightarrow \mathbb{R}
$$

using the reduced structures given by the formulas of Theorem 6.2.10. More precisely, the structure on the bundle $\tilde{\mathfrak{n}}$ is the one given by $\tilde{\nabla}^{A_{N}}, \tilde{B}^{A_{N}},[,]^{A_{N}}$. Then from Theorem 6.2.10 we obtain the formulas for the structure $\nabla, \omega,[$,$] on the$ bundle $\tilde{\mathfrak{k}} \oplus \tilde{\mathfrak{n}}$, with $\nabla \equiv \tilde{\nabla}^{A_{N}}, \omega \equiv \tilde{B}^{A_{N}},[,] \equiv[,]^{A_{N}}$.

All this can be generalized for several stages, that is, for the case where we have a chain

$$
\{e\} \equiv N_{0} \subset N_{1} \subset N_{2} \subset N_{3} \ldots \subset N_{r} \equiv G,
$$

where for each $i=0,1,2, \ldots, r-1, N_{i}$ is a normal subgroup of $N_{i+1}$. This together with some applications will be the purpose of future work. 


\section{7 \\ Further Examples}

We have already seen one example of Lagrange-Poincaré reduction, namely in the study of Wong's equations. Now we turn to some examples that involve reduction by stages. The first one concerns the classical setting of semidirect products in which one is given a Lagrangian that is invariant under the action of a semidirect product, for example, as in underwater vehicle dynamics (see Leonard and Marsden (1997)). Here we show how this theory fits into the framework of the present paper.

Following this, we consider central extensions from the Lagrangian viewpoint, the sort of example that is well known in the Hamiltonian context of the KdV equation, as in Marsden, Misiolek, Perlmutter and Ratiu [1998, 2000].

The third example is that of the spacecraft with internal rotors. The group in this case is just a direct product, but it is nonetheless interesting. See, for example, Bloch, Krishnaprasad, Marsden and Alvarez (1992).

The Lagrangian version of systems with a Lagrangian depending on parameters, in which semidirect products are, in a sense, created, is studied in the fourth example. These systems include, for instance, the classical heavy top. This sort of theory was studied in Holm, Marsden and Ratiu (1998a) and in Cendra, Holm, Marsden and Ratiu (1998).

\subsection{Semidirect Products}

In this subsection we show that the reduction of a system having a symmetry group that is a semidirect product of a Lie group $G$ with a vector space $V$ can be done in two stages, reducing by the normal subgroup $V$ first and by the group $G$ second. 
Let $G$ be a Lie group and let $\rho: G \times V \rightarrow V$ be a linear representation of $G$ on a vector space $V$. We will write, equivalently, $\rho(g, v) \equiv \rho_{g} v \equiv \rho(g) v \equiv g v$. Let $S=G \subseteq V$ be the semidirect product. Thus, by definition,

$$
\left(g_{1}, v_{1}\right)\left(g_{2}, v_{2}\right)=\left(g_{1} g_{2}, g_{1} v_{2}+v_{1}\right)
$$

and $(g, v)^{-1}=\left(g^{-1},-g^{-1} v\right)$. The Lie algebra of $S$ is $\mathfrak{s}=\mathfrak{g} \subseteq V$ with the Lie bracket given by

$$
\left[\left(\xi_{1}, v_{1}\right),\left(\xi_{2}, v_{2}\right)\right]=\left(\left[\xi_{1}, \xi_{2}\right], \rho^{\prime}\left(\xi_{1}\right) v_{2}-\rho^{\prime}\left(\xi_{2}\right) v_{1}\right) .
$$

We will think of $V$ as being a normal subgroup of $S$, as usual, via the inclusion map $V \rightarrow G(S)$ given by $v \mapsto(e, v)$. Next, we will perform the reduction of $T S=T(G(S)$ in the category $\mathfrak{L P}$ in two stages, first we reduce by $V$ and then by $G \equiv(G(S) / V$.

\section{Stage 1.}

We can identify in a natural way $T(G(S)=T G \times V \times V$. Let us choose the trivial connection $A_{V}$ on the principal bundle $G$ (S) $V$ with structure group $V$, that is $A_{V}(g, \dot{g}, v, \dot{v})=\dot{v}$. Then the curvature $B^{A_{V}}=0$. Also, by definition $V$ is Abelian and we can identify its Lie algebra $\mathfrak{v} \equiv V$, where the Lie bracket is 0 . We will also identify $(G \subseteq V) / V \equiv G$ by $[g, v]_{V} \equiv g$. The bundle $\tilde{\mathfrak{v}}$ is a trivial bundle with base $(G \subseteq V) / V \equiv G$ and we can identify $\tilde{\mathfrak{v}} \equiv G \times V$ via $[(g, v), \xi]_{V} \equiv(g, \xi)$. The covariant derivative on $\tilde{\mathfrak{v}}$ is given by

$$
\frac{\tilde{D}^{A_{V}}}{D t}[(g(t), v(t)), \xi(t)]_{V} \equiv \frac{\tilde{D}^{A_{V}}}{D t}(g(t), \xi(t))=(g(t), \dot{\xi}(t)) .
$$

We also have $\tilde{B}^{A_{V}}=0$, and $[,]^{\tilde{\mathfrak{v}}}=0$. The reduced bundle that is obtained after the first stage of reduction is then

$$
T((G \subseteq V) / V) \oplus \tilde{\mathfrak{v}} \equiv T G \times V
$$

with the structure given above.

\section{Stage 2 .}

The action of $G$ on $\tilde{\mathfrak{v}}$ commutes with the structure $\nabla \equiv \tilde{\nabla}^{A_{V}}, \omega \equiv \tilde{B}^{A_{V}}=0$, $[,] \equiv[,]^{\tilde{\mathfrak{v}}}=0$. Now consider the principal bundle $G$ over a point with structure group $G$ and connection $A_{G}(g, \dot{g})=\dot{g} g^{-1}$. We are in position to apply Theorems 6.2.10, 6.3.10 and 6.4.9. Reduction by $G$ of the bundle $T G \times V$ obtained in the first stage gives, using Theorem 6.2.10, a reduced bundle which is clearly isomorphic, in the category $\mathfrak{L P}$, to the bundle $\mathfrak{g} \oplus V$ whose base has dimension 0 . It is clear that $\omega^{\tilde{\mathfrak{g}}}=0, \nabla^{\tilde{\mathfrak{g}}}=0$, because the base has dimension 0 . Besides, we can check very easily that the formula for $[,]^{\tilde{\mathfrak{g}}}$ gives the usual semidirect product Lie algebra structure on $\mathfrak{g}(S)$. We remark that even if the bundle obtained in the first stage depends on the connection $A$, the bundle obtained in the second stage does not depend on the connection $A$. This is, of course, also a direct consequence of Theorem 6.3.10. 


\subsection{Central Extensions}

We now study the particular case of $\mathbb{R}$ or $S^{1}$-group extensions. This already includes the Bott-Virasoro central extension of $\operatorname{Diff}\left(S^{1}\right)$ as an interesting infinite dimensional example. More general central extensions by Abelian Lie groups, relative to an arbitrary action (see, for instance, De Azcárraga and Izquierdo (1995)), can also be dealt with using the methods of this paper. For instance, the examples studied in Marsden, Misiolek, Perlmutter and Ratiu [1998, 2000], such as the Heisenberg group and the Bott-Virasoro group treated there from a Hamiltonian point of view, can also be approached using our Lagrangian techniques.

Let $G$ be a Lie group and consider central extensions of $G$ of the type $\hat{G}=G \times \mathbb{R}$ or $\hat{G}=G \times S^{1}$, where the composition law is given by

$$
(g, \alpha)(h, \beta)=(g h, \alpha+\beta+\Sigma(g, h)) .
$$

If we deal with $G \times S^{1}$, the sum in second component is understood to be modulo $2 \pi$. Here, $\Sigma: G \times G \rightarrow \mathbb{R}$ is a group 2-cocycle relative to the trivial action of $G$ on $\mathbb{R}$ and hence $\Sigma$ satisfies the cocycle identity

$$
\Sigma(g, h)+\Sigma(g h, k)=\Sigma(g, h k)+\Sigma(h, k) .
$$

As is well known, the second group cohomology of $G$ with values in an Abelian group classifies the extensions of $G$ by this Abelian group. Therefore, in the definition of the composition law for $\hat{G}$ we can modify $\Sigma$ by the addition of a 2-coboundary such that $\Sigma(e, e)=0$. The cocycle identity implies that $\Sigma(h, e)=\Sigma(e, h)=\Sigma(e, e)$ and $\Sigma\left(h, h^{-1}\right)=\Sigma\left(h^{-1}, h\right)$ for all $h \in G$. It can be directly checked that the cocycle identity and the condition $\Sigma(e, e)=0$, are necessary and sufficient for the composition law defined above to satisfy the group axioms. It is easily verified that the neutral element of $\hat{G}$ is $(e, 0)$, where $e$ is the neutral element of $G$. The inverse of $(g, \alpha)$ is given by

$$
(g, \alpha)^{-1}=\left(g^{-1},-\alpha-\Sigma\left(g, g^{-1}\right)\right) .
$$

An element of the tangent space $T_{(g, \alpha)} \hat{G}$ is often denoted by $(g, \alpha, \dot{g}, \dot{\alpha})$ or $(\dot{g}, \dot{\alpha})$. The Lie algebra $\hat{\mathfrak{g}}$ is the space of all tangent vectors at $(e, 0)$, that is, vectors $(e, 0, \xi, a)$, where $\xi$ is an element of the Lie algebra $\mathfrak{g}$. With these basic formulas in place we can calculate the Lie bracket in $\hat{\mathfrak{g}}$ which is given by

$$
[(\xi, a),(\eta, b)]=\left([\xi, \eta], \partial_{1} \partial_{2} \Sigma(e, e) \cdot \eta \cdot \xi-\partial_{2} \partial_{1} \Sigma(e, e) \cdot \eta \cdot \xi\right) .
$$

Here we define

$$
\partial_{1} \partial_{2} \Sigma(e, e) \cdot \eta \cdot \xi=\left.\left.\frac{\partial}{\partial t}\right|_{t=0} \frac{\partial}{\partial s}\right|_{s=0} \Sigma(\exp t \xi, \exp s \eta)
$$

and

$$
\partial_{2} \partial_{1} \Sigma(e, e) \cdot \eta \cdot \xi=\left.\left.\frac{\partial}{\partial t}\right|_{t=0} \frac{\partial}{\partial s}\right|_{s=0} \Sigma(\exp s \eta, \exp t \xi) .
$$


We will define the Lie algebra 2-cocycle $\sigma$ by

$$
\sigma(\xi, \eta)=\partial_{1} \partial_{2} \Sigma(e, e) \cdot \eta \cdot \xi-\partial_{2} \partial_{1} \Sigma(e, e) \cdot \eta \cdot \xi
$$

and then the Lie bracket is $[(\xi, a),(\eta, b)]=([\xi, \eta], \sigma(\xi, \eta))$.

The set $N=\{(e, \alpha) \mid \alpha \in \mathbb{R}\}$ is a normal subgroup of $\hat{G}$ isomorphic to $\mathbb{R}$ or $S^{1}$. Moreover, $N$ is contained in the center of $\hat{G}$. We can therefore consider $\hat{G}$ as a principal bundle with structure group $N$. Now consider any metric on $\hat{\mathfrak{g}}$, for instance one given by the simple formula

$$
\langle(\xi, a),(\eta, b)\rangle=\langle\xi, \eta\rangle+a b,
$$

where (by a slight abuse of notation), $\langle\xi, \eta\rangle$ is a given inner product on $\mathfrak{g}$. This generates a left invariant metric on $\hat{G}$ in the following way. Let $\left(\dot{g}_{i}, \dot{\alpha}_{i}\right) \in T_{(g, \alpha)} \hat{G}$, $i=1,2$. Then define

$$
\begin{aligned}
\left\langle\left(\dot{g}_{1}, \dot{\alpha}_{1}\right),\left(\dot{g}_{2}, \dot{\alpha}_{2}\right)\right\rangle= & \left\langle g^{-1} \dot{g}_{1}, g^{-1} \dot{g}_{2}\right\rangle \\
& +\left(\dot{\alpha}_{1}+\partial_{2} \Sigma\left(g^{-1}, g\right) \cdot \dot{g}_{1}\right)\left(\dot{\alpha}_{2}+\partial_{2} \Sigma\left(g^{-1}, g\right) \cdot \dot{g}_{2}\right) .
\end{aligned}
$$

Now we can proceed to perform the construction of the mechanical connection, that is, the connection whose horizontal spaces are the orthogonal complements of the vertical spaces of the bundle $\hat{G}$ with structure group $N$. Then the conclusion of 6.3.5 holds, that is, the corresponding connection 1-form given by

$$
A_{N}(\dot{g}, \dot{\alpha})=\dot{\alpha}+\partial_{2} \Sigma\left(g^{-1}, g\right) \cdot \dot{g} .
$$

is $\hat{G}$-equivariant and not just $N$-equivariant.

Now we apply the reduction by stages process given in Theorems 6.3 .14 and 6.2.10. The first stage is reduction by $N$ and we obtain the reduced bundle $T G \oplus \tilde{\mathfrak{n}}$, since $\hat{G} / N \equiv G$. We obtain a structure $\nabla \equiv \tilde{\nabla}^{A_{N}}, B \equiv \tilde{B}^{A_{N}}$, and [,] on the bundle $\tilde{\mathfrak{n}}$. Then, by applying Theorems 6.3 .14 and 6.2 .10 we see that we obtain an equivalent expression for the Lie bracket on $\hat{\mathfrak{g}} \equiv \mathfrak{g} \oplus[\mathfrak{n}]_{G}$. We can easily check that, in the expression of the Lie bracket of Theorem 6.2.10, the terms $\left[\nabla^{(A, V)}\right]_{G \bar{\xi}_{1}}\left[v_{2}\right]_{G}$, $\left[\nabla^{(A, V)}\right]_{G \bar{\xi}_{2}}\left[v_{1}\right]_{G}$, as well as the term $\left[\left[v_{1}\right]_{G},\left[v_{2}\right]_{G}\right]$ are all 0 . Thus, in particular, the cocycle $\sigma$ is minus the reduced curvature.

\subsection{Rigid Body with Rotors}

We will use the description of the rigid body with rotors given in Marsden and Scheurle (1993b). The configuration space for a rigid body with three rotors aligned with, say, the principal axes, is

$$
Q=S O(3) \times S^{1} \times S^{1} \times S^{1}
$$

with elements denoted $\left(R, \theta_{1}, \theta_{2}, \theta_{3}\right)$, where the angles are relative to the carrier. The group structure on $Q$ is the direct product structure, so, in particular, $N=$ 
$S O(3)$ is a normal subgroup. The body angular velocity is $\Omega=R^{-1} \dot{R}$. We also denote

$$
\Omega=\left(\dot{\theta}_{1}, \dot{\theta_{2}}, \dot{\theta_{3}}\right) .
$$

We now think of $Q$ as a principal bundle with structure group $N$ and choose the mechanical connection $A_{N}$ given by

$$
A_{N}\left(R, \theta_{1}, \theta_{2}, \theta_{3}\right)=R^{-1} \dot{R}+(I+K)^{-1} K \Omega .
$$

The first stage of reduction is reduction by $N$ which gives the reduced bundle

$$
T\left(S^{1} \times S^{1} \times S^{1}\right) \oplus \tilde{\mathfrak{n}} \equiv T\left(S^{1} \times S^{1} \times S^{1}\right) \times \mathfrak{n} .
$$

The second stage is reduction by the group $S^{1} \times S^{1} \times S^{1} \equiv Q / N$. We can easily see that the reduced bundle is

$$
\mathbb{R} \times \mathbb{R} \times \mathbb{R} \times \tilde{\mathfrak{n}} / S^{1} \times S^{1} \times S^{1} \equiv \mathbb{R} \times \mathbb{R} \times \mathbb{R} \times \mathfrak{n} .
$$

We can also easily see that, in our case, several terms of the expression of the Lie bracket given in Theorem 6.2.10 vanish; more precisely, $\left[\bar{\xi}_{1}, \bar{\xi}_{2}\right]=0,\left[\nabla^{(A, V)}\right]_{G \bar{\xi}_{1}}\left[v_{2}\right]_{G}=$ $0,\left[\nabla^{(A, V)}\right]_{G \bar{\xi}_{2}}\left[v_{1}\right]_{G}=0$, and $[\omega]_{G}\left(\bar{\xi}_{1}, \bar{\xi}_{2}\right)=0$. We can also easily check that the term $\left[\left[v_{1}\right]_{G},\left[v_{2}\right]_{G}\right]$ is, in our case, simply $\left[\Omega_{1}, \Omega_{2}\right]$. Thus the Lie algebra structure on $\mathbb{R} \times \mathbb{R} \times \mathbb{R} \times \mathfrak{n}$ obtained by reduction by stages coincides with the direct product Lie algebra, as expected, according to Theorems 6.3.14 and 6.2.10.

\subsection{Systems Depending on a Parameter}

In this subsection, we consider Lagrangian systems depending on a parameter and show how they fit into the framework developed in this paper. In the process we shall recover the Euler-Poincaré equation in Holm, Marsden and Ratiu (1998a); see equation (7.4.1) below.

Recall from Marsden, Ratiu, and Weinstein (1984a) and Marsden, Misiolek, Perlmutter and Ratiu (1998) that on the Hamiltonian side of this same problem, the semidirect product $G(S)$ appears as a symmetry group of an enlarged system and symplectic reduction by stages is relevant. The situation on the Lagrangian side of the same problem is somewhat different.

The fundamental difference between this subsection and $\S 7.1$ is that in $\S 7.1$, one imagines having a system whose given symmetry group is a semidirect product from the outset, as, for example, the Euclidean group is the symmetry group of an underwater vehicle. Here, on the other hand, the Lagrangian has a symmetry group $G$, but in a way that includes the dependence on a parameter $a \in V^{*}$, where $V$ is a representation space for $G$. The goal is then to show that the more general Euler-Poincaré equations referred to above, can be obtained by using Lagrangian reduction with respect to the action of $G$.

There are interesting links between the set up described below and the topic of Clebsch variables and Lin constraints; these are described in Cendra and Marsden 
(1987). We also refer to Cendra, Holm, Marsden and Ratiu (1998) and Cendra, Holm, Hoyle and Marsden (1998) for interesting connections with degenerate Legendre transformations.

Consider a Lagrangian

$$
L: T(G \times Q) \times V^{*} \rightarrow \mathbb{R}
$$

where $G$ is a group, $Q$ is a manifold, and $V^{*}$ is the dual of the vector space $V$. The value of $L$ at the point $\left(g, q, \dot{g}, \dot{q}, a_{0}\right) \in T(G \times Q) \times V^{*}$ will be denoted $L\left(g, q, \dot{g}, \dot{q}, a_{0}\right)$, as usual, and we will think of $a_{0}$ as being a parameter that remains fixed along the evolution of the system. Assume that there is a linear action $\rho: G \times V \rightarrow V$ of $G$ on $V$, so there is also an induced action $\rho_{*}: G \times V^{*} \rightarrow V^{*}$ such that $\left\langle g a_{0}, g b_{0}\right\rangle=\left\langle a_{0}, b_{0}\right\rangle$ for all $a_{0} \in V^{*}$, all $b_{0} \in V$, and all $g \in G$, where, as usual, we write $g a_{0}=\rho\left(g, a_{0}\right)$ and $g b_{0}=\rho_{*}\left(g, b_{0}\right)$. We will often write $\left\langle a_{0}, b_{0}\right\rangle=\left\langle b_{0}, a_{0}\right\rangle$, due to the identification $V^{* *} \equiv V$. Assume that $L$ has the following invariance property:

$$
L\left(h g, q, h \dot{g}, \dot{q}, h a_{0}\right)=L\left(g, q, \dot{g}, \dot{q}, a_{0}\right) \text {, }
$$

for all $a_{0} \in V^{*}$, all $q \in Q$, and all $h, g \in G$. Let

$$
L(e, q, \xi, \dot{q}, a)=l(\xi, q, \dot{q}, a)
$$

for all $\xi \in \mathfrak{g}$, all $q \in Q$, and all $a \in V^{*}$. Then the invariance property implies

$$
L\left(g, q, \dot{g}, \dot{q}, a_{0}\right)=l(\xi, q, \dot{q}, a),
$$

for all $g \in G$, all $q \in Q$, and all $a \in V^{*}$, where $\xi=g^{-1} \dot{g}$ and $a=g^{-1} a_{0}$.

By direct calculation we can show that the following conditions are equivalent:

(i) The curve $\left(g(t), q(t), a_{0}\right)$ is a critical point of the action

$$
\int_{t_{0}}^{t_{1}} L\left(g, q, \dot{g}, \dot{q}, a_{0}\right) d t,
$$

with restrictions on variations given by $\delta g\left(t_{i}\right)=0$ for $i=0,1, \delta q\left(t_{i}\right)=0$ for $i=0,1$, and $\delta a_{0}=0$.

(ii) The curve $(\xi(t), q(t), a(t))$, where $a(t)=g^{-1}(t) a_{0}$ for all $t$ and $\xi(t)=g^{-1}(t) \dot{g}$, is a critical point of the action

$$
\int_{t_{0}}^{t_{1}} l(\xi, q, \dot{q}, a) d t,
$$

with restrictions on variations given by

$$
\delta \xi=\dot{\eta}+[\xi, \eta]
$$

where $\eta$ is any curve in $\mathfrak{g}$ such that $\eta\left(t_{i}\right)=0$ for $i=0,1$,

$$
\delta q\left(t_{i}\right)=0
$$


for $i=0,1$,

$$
\delta a=-\eta a,
$$

and

$$
\dot{a}+\xi a=0,
$$

for all $t$. We remark that this last condition comes from the condition $\dot{a}_{0}=0$ together with $a_{0}=g a$.

A direct application of (ii) leads to the equations

$$
\begin{gathered}
-\frac{d}{d t} \frac{\partial l}{\partial \xi}+\operatorname{ad}_{\xi}^{*} \frac{\partial l}{\partial \xi}+\frac{\partial l}{\partial a} \diamond a=0 \\
\frac{\partial l}{\partial q}-\frac{d}{d t} \frac{\partial l}{\partial \dot{q}}=0
\end{gathered}
$$

where for all $\eta \in \mathfrak{g}$, all $a \in V^{*}$, and all $b \in V$ we have, by definition,

$$
(b \diamond a)(\eta)=-\langle\eta a, b\rangle .
$$

The equation (7.4.1) is called the Euler-Poincaré equation (see Holm, Marsden and Ratiu (1998a)). This equation together with the Euler-Lagrange equation (7.4.2) in the variable $(q, \dot{q})$ and the equation $\dot{a}+\xi a=0$ form the complete set of equations of the system in terms of the variables $(\xi, q, a)$.

Now we shall recast conditions (i) and (ii) into an equivalent form. The idea is to introduce the condition that $a_{0}$ is a constant of the motion, that is, $\dot{a}_{0}=0$, via a Lagrange multiplier. Thus, let us define the new Lagrangian

$$
\bar{L}: T\left(G \times Q \times V^{*} \times V\right) \rightarrow \mathbb{R}
$$

by

$$
\bar{L}\left(g, q, a_{0}, b_{0}, \dot{g}, \dot{q}, \dot{a}_{0}, \dot{b}_{0}\right)=L(g, q, \dot{g}, \dot{q})+\left\langle\dot{a}_{0}, b_{0}\right\rangle .
$$

Now we observe that $G \times Q \times V^{*} \times V$ is a principal bundle with structure group $G$ acting as before, that is, $h\left(g, q, a_{0} \cdot b_{0}\right)=\left(h g, q, h a_{0}, h b_{0}\right)$. Moreover, $G \times Q \times V^{*} \times V$ is isomorphic, as a principal bundle, to the trivial bundle $G \times Q \times V^{*} \times V$ where the action of $G$ is given by

$$
h \cdot(g, q, a, b)=(h g, q, a, b),
$$

for all $h, g \in G$, all $a \in V^{*}$, and all $b \in V$. More precisely, we have the isomorphism

$$
\psi: G \times Q \times V^{*} \times V \rightarrow G \times Q \times V^{*} \times V
$$

given by

$$
\psi\left(g, q, a_{0}, b_{0}\right)=\left(g, q, g^{-1} a_{0}, g^{-1} b_{0}\right) \equiv(g, q, a, b) .
$$

We can check that

$$
\psi\left(h\left(g, q, a_{0}, b_{0}\right)\right)=h \cdot \psi\left(g, q, a_{0}, b_{0}\right) \equiv h \cdot(g, q, a, b)
$$


for all $h, g \in G$, all $a_{0} \in V^{*}$, and all $b_{0} \in V$. We can easily check that the composition $\bar{L} \circ T \psi^{-1}=: L^{V}$ is given by

$$
L^{V}(g, q, a, b, \dot{g}, \dot{q}, \dot{a}, \dot{b})=L(g, q, \dot{g}, \dot{q}, a)+\left\langle\dot{a}+g^{-1} \dot{g} a, b\right\rangle .
$$

We will use the trivial bundle, where the action of $G$ is given by the $\cdot$ operation, from now on. Thus, the base is

$$
\left(G \times Q \times V^{*} \times V\right) / G \equiv Q \times V^{*} \times V
$$

in a natural way. The Lagrangian on this bundle is $L^{V}$. Using techniques as in Cendra and Marsden (1987), which gives a version of the Lagrange multiplier theorem, we can show that conditions (i) and (ii) are equivalent to any of the following conditions

(iii) The curve $\left(g(t), q(t), a_{0}, b_{0}\right)$ is a critical point of the action

$$
\int_{t_{0}}^{t_{1}} \bar{L}\left(g, q, a_{0}, b_{0}, \dot{g}, \dot{q}, \dot{a}_{0}, \dot{b}_{0}\right) d t
$$

with restrictions on the variations given by

$$
\delta g\left(t_{i}\right)=0, \quad \delta q\left(t_{i}\right)=0, \quad \delta a_{0}\left(t_{i}\right)=0, \quad \text { and } \quad \delta b_{0}\left(t_{i}\right)=0
$$

for $i=0,1$.

(iv) The curve $(g(t), q(t), a(t), b(t))$ is a critical point of the action

$$
\int_{t_{0}}^{t_{1}} L^{V}(g, q, a, b, \dot{g}, \dot{q}, \dot{a}, \dot{b}) d t
$$

with restriction on the variations given by

$$
\delta g\left(t_{i}\right)=0, \quad \delta q\left(t_{i}\right)=0, \quad \delta a\left(t_{i}\right)=0, \quad \text { and } \quad \delta b\left(t_{i}\right)=0
$$

for $i=0,1$.

However, this time we do not want to use that version of the Lagrange multiplier theorem. Instead, we want to use directly Theorem 3.3.4 to obtain a reduced system, starting with the Lagrangian $L^{V}$, equivalent to the system

$$
\begin{aligned}
-\frac{d}{d t} \frac{\partial l}{\partial \xi}+\operatorname{ad}_{\xi}^{*} \frac{\partial l}{\partial \xi}+\frac{\partial l}{\partial a} \diamond a & =0 \\
\frac{\partial l}{\partial q}-\frac{d}{d t} \frac{\partial l}{\partial \dot{q}} & =0 \\
\dot{a}+\xi a & =0
\end{aligned}
$$

obtained before. 
We need to calculate the reduced bundle $T\left(Q \times V^{*} \times V\right) \oplus \tilde{\mathfrak{g}}$. We can easily verify that the bundle $\tilde{\mathfrak{g}}$ equals $Q \times V^{*} \times V \times \mathfrak{g}$. The Lie algebra structure on $\tilde{\mathfrak{g}}$ is given by

$$
\left[\left(q, a, b, \xi_{1}\right),\left(q, a, b, \xi_{2}\right)\right]=\left(q, a, b,\left[\xi_{1}, \xi_{2}\right]\right) .
$$

Now let us choose the trivial principal connection $A$ on $G \times Q \times V^{*} \times V$, that is, the connection given by

$$
A(g, q, a, b, \dot{g}, \dot{q}, \dot{a}, \dot{b})=\dot{g} g^{-1} .
$$

Using Lemma 2.3.4 we can see that the covariant derivative along a curve in $\tilde{\mathfrak{g}}$ is given by

$$
\frac{D}{D t}(q(t), a(t), b(t), \xi(t))=(q(t), a(t), b(t), \dot{\xi}(t)) .
$$

Therefore the connection $\tilde{\nabla}^{A}$ on $\tilde{\mathfrak{g}}$ is given by

$$
\tilde{\nabla}_{(q, a, b, \dot{q}, \dot{a}, \dot{b})}^{A}(q, a, b, \xi)=\left(q, a, b, \frac{\partial \xi}{\partial q} \dot{q} \frac{\partial \xi}{\partial a} \dot{a}+\frac{\partial \xi}{\partial b} \dot{b}\right),
$$

for any section $(q, a, b) \mapsto(q, a, b, \xi(q, a, b))$ of $\tilde{\mathfrak{g}}$ and any tangent vector

$$
(q, a, b, \dot{q}, \dot{a}, \dot{b})
$$

at the point $(q, a, b)$ of the base $Q \times V^{*} \times V$ of $\tilde{\mathfrak{g}}$. Since the curvature of $A$ is $B^{A}=0$ we have $\tilde{B}^{A}=0$. A generic element of the bundle

$$
T\left(Q \times V^{*} \times V\right) \oplus \tilde{\mathfrak{g}}
$$

will be written $(q, a, b, \dot{q}, \dot{a}, \dot{b}, \xi)$. Let $l^{V}$ be the reduced Lagrangian

$$
l^{V}: T\left(Q \times V^{*} \times V\right) \oplus \tilde{\mathfrak{g}} \rightarrow \mathbb{R} .
$$

Thus, $l^{V}(q, a, b, \dot{q}, \dot{a}, \dot{b}, \xi)$ is a function of the independent variables

$$
(q, a, b, \dot{q}, \dot{a}, \dot{b}, \xi) .
$$

More precisely, we can easily see that

$$
l^{V}(q, a, b, \dot{q}, \dot{a}, \dot{b}, \xi)=l(q, \xi, \dot{q}, a)+\langle\dot{a}+\xi a, b\rangle,
$$

where, as before,

$$
l(\xi, q, \dot{q}, a)=L(e, q, \xi, \dot{q}, a) .
$$

We can read off directly from the expression of the Lagrangian $l^{V}$ above that the condition $\dot{a}+\xi a=0$ has been imposed with the Lagrange multiplier $b$. We can write (see Cendra and Marsden (1987))

$$
l^{V}(q, a, b, \dot{q}, \dot{a}, \dot{b}, \xi)=l(q, \xi, \dot{q}, a)+J(a, b)(\xi)+\theta_{0}(a, b)(\dot{a}, \dot{b}),
$$


where $J: V^{*} \times V \rightarrow \mathfrak{g}^{*}$ is the momentum map of the lift of the action $\rho$ to the cotangent bundle $V^{*} \times V \equiv V^{*} \times V^{* *}$, and $\theta_{0}$ is the canonical 1-form on $V^{*} \times V \equiv V^{*} \times V^{* *}$. Using Theorem 3.3.4 we can obtain the vertical and also the horizontal Lagrange-Poincaré equations. The vertical Lagrange-Poincaré equation is

$$
-\frac{d}{d t}\left(\frac{\partial l}{\partial \xi}(\xi, q, \dot{q}, a)+J(a, b)\right)+\operatorname{ad}_{\xi}^{*}\left(\frac{\partial l}{\partial \xi}(\xi, q, \dot{q}, a)+J(a, b)\right)=0 .
$$

The horizontal Lagrange-Poincaré equation is

$$
\begin{aligned}
\frac{\partial l}{\partial q}(\xi, q, \dot{q}, a)-\frac{d}{d t} \frac{\partial l}{\partial \dot{q}}(\xi, q, \dot{q}, a) & =0 \\
\frac{\partial l}{\partial a}(\xi, q, \dot{q}, a)+\frac{\partial J(a, b)(\xi)}{\partial a}-\frac{d b}{d t} & =0 \\
\dot{a}+\xi a & =0 .
\end{aligned}
$$

Using the property $\langle\xi a, b\rangle+\langle a, \xi b\rangle=0$, we can see that

$$
\frac{\partial J(a, b)(\xi)}{\partial a}=-\xi b
$$

The last two horizontal equations can therefore be rewritten as

$$
\begin{aligned}
& \dot{b}=-\xi b+\frac{\partial l}{\partial a} \\
& \dot{a}=-\xi a .
\end{aligned}
$$

It is also clear that

$$
\frac{d J(a, b)}{d t}=d J(a, b)(\dot{a}, \dot{b})
$$

Thus we obtain

$$
\frac{d J(a, b)}{d t}=d J(a, b)\left(-\xi a,-\xi b+\frac{\partial l}{\partial a}\right) .
$$

Using directly the formula $J(a, b)(\nu)=\langle\nu a, b\rangle=-\langle a, \nu b\rangle$ we can prove in a straightforward manner that

$$
d J(a, b)\left(-\xi a,-\xi b+\frac{\partial l}{\partial a}\right)=\operatorname{ad}_{\xi}^{*} J(a, b)-\frac{\partial l}{\partial a} \diamond a .
$$

Using this, together with the vertical Lagrange-Poincaré equation, we obtain at each point $(\xi, q, \dot{q}, a)$,

$$
-\frac{d}{d t}\left(\frac{\partial l}{\partial \xi}\right)+\frac{\partial l}{\partial a} \diamond a+\operatorname{ad}_{\xi}^{*}\left(\frac{\partial l}{\partial \xi}\right)=0,
$$

which is the Euler-Poincaré equation. We can easily conclude from all this that any solution to the reduced system of equations is a solution of the system (7.4.3). 
We can also prove the converse, namely, starting with a solution $(q(t), a(t), \xi(t))$ of the system (7.4.3) we can see that the curve $b(t)=g(t) b_{0}$, for any given $b_{0} \in V$, is such that $(q(t), a(t), b(t), \xi(t))$ is a solution of the system formed by the reduced vertical and horizontal Euler-Poincaré equations. This is done by simply reversing the previous procedure. 
7. Further Examples 


\section{8 \\ The Category $\mathfrak{L P}^{*}$ and Poisson Geometry}

In this section we will define a new category $\mathfrak{L P}^{*}$. The objects of $\mathfrak{L P}^{*}$ are the dual bundles of the objects in $\mathfrak{L P}$ and they carry a Poisson structure which is dual to the Lie bracket structure on sections of objects of the category $\mathfrak{L P}$. Cotangent bundles are important examples of objects of $\mathfrak{L P}^{*}$. The parallelism between Lagrangian mechanics and Poisson mechanics is a consequence of the Legendre transformation. Interesting aspects of this parallelism can be viewed under the light of the categorical duality. For instance, reduction in the category $\mathfrak{L P}^{*}$ is dual to reduction in the category $\mathfrak{L P}$. This includes cotangent bundle reduction as a special case (see Montgomery, Marsden and Ratiu (1984) and Montgomery (1986)). Thus, $\mathfrak{L P}^{*}$ is an interesting class of Poisson manifolds which is stable under reduction.

In this section we establish the basic link between Lagrangian and Poisson mechanics given by this duality and show how reduction in the category $\mathfrak{L P}^{*}$, which is a subcategory of the category of Poisson manifolds and Poisson maps, can be viewed as being dual to reduction in the category $\mathfrak{L P}$. A more complete study, including a precise description of the symplectic leaves of objects of $\mathfrak{L P}^{*}$ and several other related topics will be the purpose of a future work.

\subsection{The Poisson Bracket on Duals of Objects of $\mathfrak{L P}$}

We shall begin with some notation and definitions. Let $W=T Q \oplus V$ be an object of $\mathfrak{L P}$ and let $\pi: W \rightarrow Q, p_{Q}: W \rightarrow T Q$ and $p_{V}: W \rightarrow V$ be the natural projections. 
Let $W^{*}=(T Q \oplus V)^{*} \equiv T^{*} Q \oplus V^{*}$ be the dual of $W$ and let

$$
\bar{\pi}: W^{*} \rightarrow Q, \quad \bar{p}_{Q}: W^{*} \rightarrow T^{*} Q, \quad \text { and } \quad \bar{p}_{V}: W^{*} \rightarrow V^{*}
$$

be the naturally induced projections. A section $w \in \Gamma(W)$ will be sometimes written more explicitly as $w=X \oplus v$ or $w=\dot{q} \oplus v$. Likewise, a section $\mu \in \Gamma\left(W^{*}\right)$ will be sometimes written $\mu=\gamma \oplus \nu$. We have well defined maps

$$
\Gamma\left(p_{Q}\right): \Gamma(W) \rightarrow \Gamma(T Q) \quad \text { and } \quad \Gamma\left(p_{V}\right): \Gamma(W) \rightarrow \Gamma(V)
$$

and also

$$
\Gamma\left(\bar{p}_{Q}\right): \Gamma\left(W^{*}\right) \rightarrow \Gamma\left(T^{*} Q\right) \quad \text { and } \quad \Gamma\left(\bar{p}_{V}\right): \Gamma\left(W^{*}\right) \rightarrow \Gamma\left(V^{*}\right) .
$$

We will often denote

$$
\Gamma\left(p_{Q}\right)(w) \equiv p_{Q} w \quad \text { and } \quad \Gamma\left(p_{V}\right)(w) \equiv p_{V} w
$$

and also

$$
\Gamma\left(\bar{p}_{Q}\right) \mu \equiv \bar{p}_{Q} \mu \quad \text { and } \quad \Gamma\left(\bar{p}_{V}\right)(\mu) \equiv \bar{p}_{V} \mu .
$$

Thus, if we write a section $w \in \Gamma(W)$ as $w=X \oplus v$ and a section $\mu \in \Gamma\left(W^{*}\right)$ as $\mu=\gamma \oplus \nu$, we have

$$
\Gamma\left(p_{Q}\right)(w) \equiv p_{Q} w=X \quad \text { and } \quad \Gamma\left(p_{V}\right)(w) \equiv p_{V} w=v
$$

and also

$$
\Gamma\left(\bar{p}_{Q}\right) \mu \equiv \bar{p}_{Q} \mu=\gamma \quad \text { and } \quad \Gamma\left(\bar{p}_{V}\right)(\mu) \equiv \bar{p}_{V} \mu=\nu .
$$

Definition 8.1.1. For each $w \in \Gamma(W)$ we define the function $P(w) \in C^{\infty}\left(W^{*}\right)$ by

$$
P(w)(\mu)=\mu(w)
$$

for all $\mu \in W^{*}$. In addition, for each $f \in C^{\infty}(Q)$ we have the function $f \circ \bar{\pi} \in$ $C^{\infty}\left(W^{*}\right)$.

Define the space $\mathrm{A}\left(W^{*}\right) \subset C^{\infty}\left(W^{*}\right)$ to be the vector space generated by the set of all functions $f \circ \bar{\pi}$ with $f \in C^{\infty}(Q)$, together with the set of all functions that are linear along the fibers of $C^{\infty}\left(W^{*}\right)$, that is, the functions of the type $P(w)$ with $w \in \Gamma(W)$.

Lemma 8.1.2. Let $w_{i} \in \Gamma(W)$ and $f_{i} \in C^{\infty}(Q)$, for $i=1,2$. Define We will sometimes write, for given $f \in C^{\infty}(Q)$ and given $w \in \Gamma(W), f \equiv f \circ \bar{\pi}$ and $w[f] \equiv\left(\Gamma\left(p_{Q}\right)(w)\right)[f] \circ \bar{\pi}$, for short.

Then $\{$,$\} extends to a uniquely determined skew-symmetric bilinear map$

$$
\{,\}: \mathrm{A}\left(W^{*}\right) \times \mathrm{A}\left(W^{*}\right) \rightarrow \mathrm{A}\left(W^{*}\right) .
$$

The operation $\{$,$\} satisfies the Jacobi identity, that is,$

$$
\left\{f_{1},\left\{f_{2}, f_{3}\right\}\right\}=\left\{\left\{f_{1}, f_{2}\right\}, f_{3}\right\}+\left\{f_{2},\left\{f_{1}, f_{3}\right\}\right\}
$$

for all $f_{i} \in \mathrm{A}\left(W^{*}\right), i=1,2,3$. 
Proof. Every element of $\mathrm{A}\left(W^{*}\right)$ can be written $f \circ \bar{\pi}+P(w)$ for some uniquely determined $f \in C^{\infty}(Q)$ and $w \in \Gamma(W)$. Using this we can prove in a straightforward manner that $\{$,$\} extends to a uniquely determined skew-symmetric bilinear$ form on $\mathrm{A}\left(W^{*}\right)$. The verification that $\{$,$\} satisfies the Jacobi identity is also a$ straightforward calculation.

Theorem 8.1.3. The operation $\{$,$\} on \mathrm{A}\left(W^{*}\right)$ defined in the previous lemma can be uniquely extended to a Poisson bracket

$$
\{,\}: C^{\infty}\left(W^{*}\right) \times C^{\infty}\left(W^{*}\right) \rightarrow C^{\infty}\left(W^{*}\right) .
$$

Proof. The proof will be divided into several steps.

\section{Step 1:}

The definition of the bracket.

Let $f_{i} \in C^{\infty}\left(W^{*}\right), i=1,2$, and $\sigma_{0} \in W^{*}$ be given and denote $\bar{\pi}\left(\sigma_{0}\right)=q_{0}$. We are going to define $\left\{f_{1}, f_{2}\right\}\left(\sigma_{0}\right)$. Consider any section $\sigma \in \Gamma\left(W^{*}\right)$ such that $\sigma\left(q_{0}\right)=\sigma_{0}$. Then for each $q \in Q$ we have the Taylor expansion, for $i=1,2$,

$$
f_{i}(\mu)=f_{i}(\sigma(q))+\frac{\partial f_{i}}{\partial \mu}(\sigma(q))(\mu-\sigma(q))+\epsilon_{i}(\sigma(q), \mu) \cdot(\mu-\sigma(q)),
$$

where for each $q \in Q$ we have $\mu \in W_{q}^{*}$,

$$
\frac{\partial f_{i}}{\partial \mu}(\sigma(q))
$$

is the fiber derivative of $f_{i}$ along $W_{q}^{*}$ evaluated at $\sigma(q)$, and $\epsilon_{i}(\sigma(q), \mu) \rightarrow 0$ as $\mu \rightarrow \sigma(q)$. Define $w_{i} \in \Gamma(W)$ and $a_{i} \in C^{\infty}(Q)$ for $i=1,2$, by

$$
w_{i}(q)=\frac{\partial f_{i}}{\partial \mu}(\sigma(q))
$$

and

$$
a_{i}(q)=f_{i}(\sigma(q))-\frac{\partial f_{i}}{\partial \mu}(\sigma(q)) \sigma(q)
$$

for all $q \in Q$. Thus, for $i=1,2, a_{i}+P\left(w_{i}\right)$ is the affine approximation of $f_{i}$ along the fibers of $W^{*}$. To emphasize the dependence on $\sigma$ we will sometimes write $a_{i}^{\sigma} \equiv a_{i}$, $w_{i}^{\sigma} \equiv w_{i}$, and $f_{i}^{\sigma} \equiv a_{i}^{\sigma}+P\left(w_{i}^{\sigma}\right)$, for $i=1,2$. The functions $f_{i}^{\sigma}$ are elements of $\mathrm{A}\left(W^{*}\right)$. Then we can define, for each choice of $\sigma$ satisfying $\sigma_{0}=\sigma\left(q_{0}\right)$,

$$
\left\{f_{1}, f_{2}\right\}^{\sigma, \sigma_{0}}(\mu)=\left\{f_{1}^{\sigma}, f_{2}^{\sigma}\right\}(\mu),
$$

where the bracket on the right hand side is the bracket in $\mathrm{A}\left(W^{*}\right)$ defined before. We want to show that

$$
\left\{f_{1}, f_{2}\right\}^{\sigma, \sigma_{0}}\left(\sigma_{0}\right)
$$

only depends on $\sigma_{0}$ and not on $\sigma$. To do this, we need first the following technical statement. 


\section{Step 2:}

If $\mathbf{d} f_{i}\left(\sigma_{0}\right)=0$ for $i=1$ or $i=2$, then $\left\{f_{1}, f_{2}\right\}^{\sigma, \sigma_{0}}\left(\sigma_{0}\right)=0$.

We work in a local bundle chart of $W=T Q \oplus V$ whose restriction to $T Q$ is a natural tangent bundle chart. This can be done by first choosing a vector bundle chart of $V$ which induces a chart of $Q$ and hence has a naturally associated tangent bundle chart. The same bundle chart of $W$ induces a bundle chart on $W^{*}=T^{*} Q \oplus V^{*}$. An element of $W$ will be represented in this local chart by $(q, w) \equiv(q, \dot{q} \oplus v)$ and an element of $W^{*}$ will be represented in the corresponding local chart by $(q, \mu) \equiv(q, p \oplus \nu)$. Thus we will write, with a slight abuse of notation,

$$
\begin{gathered}
\sigma_{0}=\left(q_{0}, \mu_{0}\right)=\left(q_{0}, p_{0} \oplus \nu_{0}\right), \\
\sigma(q)=(q, \mu(q))=(q, p(q) \oplus \nu(q)),
\end{gathered}
$$

and

$$
w_{i}^{\sigma}(q) \equiv\left(q, w_{i}^{\sigma}(q)\right) \equiv\left(q, \dot{q}_{i}^{\sigma}(q) \oplus v_{i}^{\sigma}(q)\right) .
$$

A straightforward calculation shows that, for $i=1,2$, we have $\mathbf{d} f_{i}\left(\sigma_{0}\right)=\mathbf{d} f_{i}^{\sigma}\left(\sigma_{0}\right)$. It is also easy to see that $\mathbf{d} f_{i}^{\sigma}\left(\sigma_{0}\right)=0$ for $i=1$ or $i=2$ if and only if the following equalities hold, for $i=1$ or $i=2$, respectively :

$$
\begin{aligned}
\frac{\partial a_{i}^{\sigma}}{\partial q}\left(q_{0}\right)+\mu_{0} \frac{\partial w_{i}^{\sigma}}{\partial q}\left(q_{0}\right) & =0 \\
w_{i}^{\sigma}\left(q_{0}\right) & =0
\end{aligned}
$$

or, equivalently, for $i=1,2$,

$$
\begin{aligned}
\frac{\partial a_{i}^{\sigma}}{\partial q}\left(q_{0}\right)+p_{0} \frac{\partial \dot{q}_{i}^{\sigma}}{\partial q}\left(q_{0}\right)+\nu_{0} \frac{\partial v_{i}^{\sigma}}{\partial q}\left(q_{0}\right) & =0 \\
\dot{q}_{i}^{\sigma}\left(q_{0}\right) & =0 \\
v_{i}^{\sigma}\left(q_{0}\right) & =0
\end{aligned}
$$

This is valid for any chart as before. From now on we will assume that the previous conditions are satisfied for $i=1$. The case $i=2$ can be established in an entirely analogous way. By definition we have

$$
\begin{aligned}
\left\{f_{1}, f_{2}\right\}^{\sigma, \sigma_{0}}\left(\sigma_{0}\right) & =\left\{f_{1}^{\sigma}, f_{2}^{\sigma}\right\}\left(\sigma_{0}\right) \\
& =\left\{a_{1}^{\sigma}+P\left(w_{1}^{\sigma}\right), a_{2}^{\sigma}+P\left(w_{2}^{\sigma}\right)\right\}\left(\sigma_{0}\right) \\
& =w_{2}^{\sigma}\left(a_{1}^{\sigma}\right)\left(q_{0}\right)-w_{1}^{\sigma}\left(a_{2}^{\sigma}\right)\left(q_{0}\right)-P\left(\left[w_{1}^{\sigma}, w_{2}^{\sigma}\right]\right)\left(\sigma_{0}\right) .
\end{aligned}
$$

Since $w_{1}^{\sigma}\left(q_{0}\right)=0$ we have $w_{1}^{\sigma}\left(q_{0}\right)\left(a_{2}^{\sigma}\right)\left(q_{0}\right)=0$. On the other hand, by definition, we have

$$
\begin{aligned}
w_{2}^{\sigma}\left(q_{0}\right)\left(a_{1}^{\sigma}\right)\left(q_{0}\right) & =\left(p_{Q} w_{2}^{\sigma}\right)\left(a_{1}^{\sigma}\right)\left(q_{0}\right) \\
& =\frac{\partial a_{1}^{\sigma}}{\partial q}\left(q_{0}\right)\left(p_{Q} w_{2}^{\sigma}\right)\left(q_{0}\right) \\
& =\frac{\partial a_{1}^{\sigma}}{\partial q}\left(q_{0}\right) \dot{q}_{2}^{\sigma}\left(q_{0}\right) .
\end{aligned}
$$


By Definition 6.1.1 the Lie bracket in the category $\mathfrak{L P}$ is given by

$$
\begin{aligned}
{\left[w_{1}^{\sigma}, w_{2}^{\sigma}\right] } & =\left[\dot{q}_{1}^{\sigma} \oplus v_{1}^{\sigma}, \dot{q}_{2}^{\sigma} \oplus v_{2}^{\sigma}\right] \\
& =\left[\dot{q}_{1}^{\sigma}, \dot{q}_{2}^{\sigma}\right] \oplus \nabla_{\dot{q}_{1}^{\sigma}} v_{2}^{\sigma}-\nabla_{\dot{q}_{2}^{\sigma}} v_{1}^{\sigma}-\omega\left(\dot{q}_{1}^{\sigma}, \dot{q}_{2}^{\sigma}\right)+\left[v_{1}^{\sigma}, v_{2}^{\sigma}\right]
\end{aligned}
$$

Since $\dot{q}_{1}^{\sigma}\left(q_{0}\right)=0$ and $v_{1}^{\sigma}\left(q_{0}\right)=0$ we have

$$
\left[w_{1}^{\sigma}, w_{2}^{\sigma}\right]\left(q_{0}\right)=\left[\dot{q}_{1}^{\sigma}, \dot{q}_{2}^{\sigma}\right]\left(q_{0}\right) \oplus-\nabla_{\dot{q}_{2}^{\sigma}} v_{1}^{\sigma}\left(q_{0}\right)
$$

We can easily show that

$$
\left[\dot{q}_{1}^{\sigma}, \dot{q}_{2}^{\sigma}\right]\left(q_{0}\right)=-\frac{\partial \dot{q}_{1}^{\sigma}}{\partial q}\left(q_{0}\right) \dot{q}_{2}^{\sigma}\left(q_{0}\right)
$$

Now we choose the chart on $W$ in such a way that the Christoffel symbols of the connection $\nabla$ on $V$ vanish at $q_{0}$, which we can assume without any loss of generality. Then we obtain

$$
\nabla_{\dot{q}_{2}^{\sigma}} v_{1}^{\sigma}\left(q_{0}\right)=\frac{\partial v_{1}^{\sigma}}{\partial q}\left(q_{0}\right) \dot{q}_{2}^{\sigma}\left(q_{0}\right) .
$$

Therefore, using the previous equalities, we can conclude that

$$
\begin{aligned}
\left\{f_{1}^{\sigma}, f_{2}^{\sigma}\right\}\left(\sigma_{0}\right) & =\frac{\partial a_{1}^{\sigma}}{\partial q}\left(q_{0}\right) \dot{q}_{2}^{\sigma}\left(q_{0}\right)+p_{0} \frac{\partial \dot{q}_{1}^{\sigma}}{\partial q}\left(q_{0}\right) \dot{q}_{2}^{\sigma}\left(q_{0}\right)+\nu_{0} \frac{\partial v_{1}^{\sigma}}{\partial q}\left(q_{0}\right) \dot{q}_{2}^{\sigma}\left(q_{0}\right) \\
& =\left(\frac{\partial a_{1}^{\sigma}}{\partial q}\left(q_{0}\right)+p_{0} \frac{\partial \dot{q}_{1}^{\sigma}}{\partial q}\left(q_{0}\right)+\nu_{0} \frac{\partial v_{1}^{\sigma}}{\partial q}\left(q_{0}\right)\right) \dot{q}_{2}^{\sigma}\left(q_{0}\right) \\
& =0 .
\end{aligned}
$$

We have therefore proved the aforementioned property, namely $\mathbf{d} f_{i}\left(\sigma_{0}\right)=0$ for $i=1$ or $i=2$ implies that $\left\{f_{1}, f_{2}\right\}^{\sigma, \sigma_{0}}\left(\sigma_{0}\right)=0$.

\section{Step 3:}

The bracket is independent of $\sigma$.

Using the above property, it follows by a standard argument that for given $f_{i}, g_{i} \in$ $C^{\infty}\left(W^{*}\right), i=1,2$, and $\sigma \in \Gamma\left(W^{*}\right)$ satisfying $\mathbf{d} f_{i}\left(\sigma_{0}\right)=\mathbf{d} g_{i}\left(\sigma_{0}\right)$ and $\sigma\left(q_{0}\right)=\sigma_{0}$, we have

$$
\left\{f_{1}, f_{2}\right\}^{\sigma, \sigma_{0}}\left(\sigma_{0}\right)=\left\{g_{1}, g_{2}\right\}^{\sigma, \sigma_{0}}\left(\sigma_{0}\right) \text {. }
$$

We can easily check that if $f_{i} \in \mathrm{A}\left(W^{*}\right)$, for $i=1,2$, then for any section $\sigma \in \Gamma\left(W^{*}\right)$ and any choice of $\sigma_{0}$ such that $\sigma\left(q_{0}\right)=\sigma_{0}$ we have $f_{i}=f_{i}^{\sigma}$, for $i=1,2$, and also

$$
\left\{f_{1}, f_{2}\right\}\left(\sigma_{0}\right)=\left\{f_{1}, f_{2}\right\}^{\sigma, \sigma_{0}}\left(\sigma_{0}\right)
$$

On the other hand, we can show, using the Taylor expansion, that for any choice of a section $\sigma^{\prime} \in \Gamma\left(W^{*}\right)$ satisfying $\sigma^{\prime}\left(q_{0}\right)=\sigma_{0}$ and any $f \in C^{\infty}\left(W^{*}\right)$ we have 
$\mathbf{d} f^{\sigma^{\prime}}\left(\sigma_{0}\right)=\mathbf{d} f\left(\sigma_{0}\right)=\mathbf{d} f^{\sigma}\left(\sigma_{0}\right)$. Using all this and the previous step, we can see that

$$
\begin{aligned}
\left\{f_{1}, f_{2}\right\}^{\sigma, \sigma_{0}}\left(\sigma_{0}\right) & =\left\{f_{1}^{\sigma}, f_{2}^{\sigma}\right\}^{\sigma, \sigma_{0}}\left(\sigma_{0}\right) \\
& =\left\{f_{1}^{\sigma^{\prime}}, f_{2}^{\sigma^{\prime}}\right\}^{\sigma, \sigma_{0}}\left(\sigma_{0}\right)=\left\{f_{1}^{\sigma^{\prime}}, f_{2}^{\sigma^{\prime}}\right\}^{\sigma^{\prime}, \sigma_{0}}\left(\sigma_{0}\right)=\left\{f_{1}, f_{2}\right\}^{\sigma^{\prime}, \sigma_{0}}\left(\sigma_{0}\right) .
\end{aligned}
$$

So far, we have proved that the bracket $\left\{f_{1}, f_{2}\right\}^{\sigma, \sigma_{0}}\left(\sigma_{0}\right)$ is well defined, that is, it does not depend on the choice of the section $\sigma \in \Gamma\left(W^{*}\right)$ as long as $\sigma\left(q_{0}\right)=\sigma_{0}$. Thus, we shall denote this bracket from now on only by $\left\{f_{1}, f_{2}\right\}\left(\sigma_{0}\right)$.

It is clear that the well defined operation $\left\{f_{1}, f_{2}\right\}\left(\sigma_{0}\right)$ is bilinear and skew symmetric. We need to show that it satisfies the Jacobi identity.

\section{Step 4:}

Verification of the Jacobi identity.

Let $f_{i} \in C^{\infty}\left(W^{*}\right), i=1,2,3$, and $\sigma_{0} \in W^{*}$. We need to verify that

$$
\left\{f_{1},\left\{f_{2}, f_{3}\right\}\right\}\left(\sigma_{0}\right)=\left\{\left\{f_{1}, f_{2}\right\}, f_{3}\right\}\left(\sigma_{0}\right)+\left\{f_{2},\left\{f_{1}, f_{3}\right\}\right\}\left(\sigma_{0}\right) .
$$

To do this, choose $\sigma \in \Gamma\left(W^{*}\right)$ such that $\sigma\left(q_{0}\right)=\sigma_{0}$. Using what we have proven so far we can see that the question reduces itself to showing that

$$
\left\{f_{1}^{\sigma},\left\{f_{2}^{\sigma}, f_{3}^{\sigma}\right\}\right\}\left(\sigma_{0}\right)=\left\{\left\{f_{1}^{\sigma}, f_{2}^{\sigma}\right\}, f_{3}^{\sigma}\right\}\left(\sigma_{0}\right)+\left\{f_{2}^{\sigma},\left\{f_{1}^{\sigma}, f_{3}^{\sigma}\right\}\right\}\left(\sigma_{0}\right) .
$$

However, this identity holds by Lemma 8.1.2 since $f_{i}^{\sigma} \in \mathrm{A}\left(W^{*}\right)$, for $i=1,2,3$.

The last axiom for a Poisson bracket is the Leibnitz identity. We shall verify it below.

\section{Step 5:}

Verification of the Leibnitz identity.

Let $f_{i} \in C^{\infty}\left(W^{*}\right), i=1,2,3$, and $\sigma_{0} \in W^{*}$. We must prove that

$$
\left\{f_{1}, f_{2} f_{3}\right\}\left(\sigma_{0}\right)=\left\{f_{1}, f_{2}\right\}\left(\sigma_{0}\right) f_{3}\left(\sigma_{0}\right)+f_{2}\left(\sigma_{0}\right)\left\{f_{1}, f_{3}\right\}\left(\sigma_{0}\right) .
$$

As we have seen before, we can assume without loss of generality that $f_{i}=f_{i}^{\sigma} \in$ $\mathrm{A}\left(W^{*}\right)$, for $i=1,2,3$, so we can write $f_{i}=a_{i}+P\left(w_{i}\right)$, for $a_{i} \in C^{\infty}(Q)$ and $w_{i} \in \Gamma(W)$. Using the bilinearity and skew-symmetry of $\{$,$\} , we can easily see that$ it is sufficient to consider the following six particular cases:

$$
\begin{aligned}
& f_{1} \equiv a_{1}, f_{2} \equiv a_{2}, f_{3} \equiv a_{3} ; \\
& f_{1} \equiv a_{1}, f_{2} \equiv a_{2}, f_{3} \equiv P\left(w_{3}\right) ; \\
& f_{1} \equiv a_{1}, f_{2} \equiv P\left(w_{2}\right), f_{3} \equiv P\left(w_{3}\right) ; \\
& f_{1} \equiv P\left(w_{1}\right), f_{2} \equiv a_{2}, f_{3} \equiv a_{3} ; \\
& f_{1} \equiv P\left(w_{1}\right), f_{2} \equiv a_{2}, f_{3} \equiv P\left(w_{3}\right) ; \\
& f_{1} \equiv P\left(w_{1}\right), f_{2} \equiv P\left(w_{2}\right), f_{3} \equiv P\left(w_{3}\right) .
\end{aligned}
$$


The first, second, fourth, and fifth cases can be established using directly the definition of the bracket on $\mathrm{A}\left(W^{*}\right)$ and the fact that for any $a \in C^{\infty}(Q)$ and any $w \in \Gamma(W)$ we have $a P(w)=P(a w)$.

To prove the third case, we first write the Taylor expansion of $P\left(w_{2}\right)$ and $P\left(w_{3}\right)$, namely

$$
P\left(w_{i}\right)(q, \mu)=\sigma(q)\left(w_{i}\right)(q)+(\mu-\sigma(q))\left(w_{i}\right)(q)
$$

for $i=2,3$. Then, since

$$
\mathbf{d}\left((\mu-\sigma(q))\left(w_{2}\right)(q) \cdot(\mu-\sigma(q))\left(w_{3}\right)(q)\right)=0
$$

at $(q, \mu)=\sigma_{0}$, we see that

$$
\begin{aligned}
\left\{a_{1}, P\left(w_{2}\right) P\left(w_{3}\right)\right\}\left(\sigma_{0}\right) & =\left\{a_{1}, \sigma(q)\left(w_{2}\right)(q) P\left(w_{3}\right)+P\left(w_{2}\right) \sigma(q)\left(w_{3}\right)(q)\right\}\left(\sigma_{0}\right) \\
& =\left\{a_{1}, P\left(\sigma(q)\left(w_{2}\right)(q) w_{3}\right)+P\left(\sigma(q)\left(w_{3}\right)(q) w_{2}\right)\right\}\left(\sigma_{0}\right)
\end{aligned}
$$

The Leibnitz identity for this case now follows from this equality.

To prove the sixth case we use the Taylor expansion of $P\left(w_{2}\right)$ and $P\left(w_{3}\right)$. We obtain

$$
\begin{aligned}
& \left\{P\left(w_{1}\right), P\left(w_{2}\right) P\left(w_{3}\right)\right\}\left(\sigma_{0}\right) \\
& \quad=\left\{P\left(w_{1}\right), \sigma(q)\left(w_{2}\right)(q) P\left(w_{3}\right)+P\left(w_{2}\right) \sigma(q)\left(w_{3}\right)(q)\right\}\left(\sigma_{0}\right) \\
& \quad=\left\{P\left(w_{1}\right), P\left(\sigma(q)\left(w_{2}\right)(q) w_{3}\right)+P\left(\sigma(q)\left(w_{3}\right)(q) w_{2}\right)\right\}\left(\sigma_{0}\right) .
\end{aligned}
$$

As before, this equality implies the Leibnitz identity for this case also.

\section{Remark.}

In the extreme case $V=0$, that is, $W \equiv T Q$, we have $W^{*}=T^{*} Q$ and the Poisson structure on $W^{*}$ coincides with the standard Poisson structure on the symplectic manifold $T^{*} Q$. In the other extreme case in which $Q$ is a point, that is $W \equiv V$, we have $W^{*}=V^{*}$ and the Poisson structure on $W^{*}$ coincides with the standard Lie-Poisson structure on the dual of a Lie algebra.

\subsection{Poisson Reduction in the Category $\mathfrak{L P}^{*}$ Viewed as Dual to Reduction in the Category $\mathfrak{L P}$}

Let $W=T Q \oplus V$ be an object of $\mathfrak{L P}$ and assume that the hypotheses of Theorem 6.2.10 hold. The composition of the natural vector bundle map $\pi_{G}(W): W \rightarrow W / G$ and the vector bundle isomorphism

$$
\alpha_{A}^{W}:(T Q \oplus V) / G \rightarrow T(Q / G) \oplus \tilde{\mathfrak{g}} \oplus(V / G),
$$


of Definition 6.2.11 gives a vector bundle map $P_{G}(W)=\alpha_{A}^{W} \circ \pi_{G}(W)$. Introducing the notation

$$
W_{G}=T(Q / G) \oplus \tilde{\mathfrak{g}} \oplus(V / G)
$$

for the target space, $W_{G}$ is an object of $\mathfrak{L P}$, and

$$
P_{G}(W): W \rightarrow W_{G}
$$

is a morphism of $\mathfrak{L P}$. The restriction of $P_{G}(W)$ to the zero section coincides with the natural projection $\pi_{G}(Q): Q \rightarrow Q / G$ and the restriction of $P_{G}(W)$ to each fiber is a linear isomorphism. Thus, $P_{G}(W)$ induces a vector bundle morphism $\bar{P}(W)_{G}: W^{*} \rightarrow W_{G}^{*}$. The restriction of $\bar{P}(W)_{G}$ to the zero section coincides with the natural projection $\pi_{G}(Q): Q \rightarrow Q / G$. The restriction of $\bar{P}(W)_{G}$ to each fiber is a linear isomorphism, which is precisely the dual of the inverse of the restriction of $P_{G}(W)$ to the same fiber.

According to Theorem 8.1.3, $W^{*}$ and $W_{G}^{*}$ each carry a Poisson structure. We are going to show that $\bar{P}(W)_{G}$ is a Poisson map.

We begin by introducing some notation. Reduced objects under the action of $G$ will be denoted with a subindex ${ }_{G}$. Let us give more precise definitions. For given $w \in W$ we will denote $w_{G}=P_{G}(W)(w)$. Likewise, for $\mu \in W^{*}$ we will denote $\mu_{G}=\bar{P}(W)_{G}(\mu)$. If $w \in \Gamma^{G}(W)$ or $\mu \in \Gamma^{G}\left(W^{*}\right)$ then, respectively, $w_{G} \in \Gamma\left(W_{G}\right)$ is the unique section of $W_{G}$ such that

$$
P_{G}(W)(w(q))=w_{G}(\pi(q))
$$

for all $q \in Q$ and $\mu_{G} \in \Gamma\left(W_{G}^{*}\right)$ is the unique section of $W_{G}^{*}$ such that

$$
\bar{P}(W)_{G}(\mu(q))=\mu_{G}(\pi(q)) \text { for all } q \in Q .
$$

If $f \in C^{\infty}\left(W^{*}\right)$ is $G$-invariant or $a \in C^{\infty}(Q)$ is $G$-invariant then, respectively, $f_{G} \in$ $C^{\infty}\left(W_{G}^{*}\right)$ is the unique function such that $f=f_{G} \circ \bar{P}(W)_{G}$ and $a_{G} \in C^{\infty}(Q / G)$ is the unique function such that $a=a_{G} \circ \pi_{G}(Q)$. We can easily show that, for any $w \in W$ and any $\mu \in W^{*}$ we have $\mu_{G}\left(w_{G}\right)=\mu(w)$. For given $w_{i} \in \Gamma^{G}(W)$, $i=1,2$, we have proven before (this is part of the content of Theorem 6.2.10) that $\left[w_{1 G}, w_{2 G}\right]=\left[w_{1}, w_{2}\right]_{G}$. It is also easy to see that for given $w \in \Gamma^{G}(W)$ and $a \in C^{\infty}(Q)$, a $G$-invariant function, we have $w_{G}\left(a_{G}\right)=(w(a))_{G}$. Using the last two assertions we can easily show that for any given $w_{i} \in \Gamma^{G}(W), i=1,2$, we have

$$
\left\{P\left(w_{1 G}\right), P\left(w_{2 G}\right)\right\}=\left\{P\left(w_{1}\right), P\left(w_{2}\right)\right\}_{G},
$$

that is, for any $\sigma \in W^{*}$ we have

$$
\left\{P\left(w_{1 G}\right), P\left(w_{2 G}\right)\right\}\left(\sigma_{G}\right)=\left\{P\left(w_{1}\right), P\left(w_{2}\right)\right\}_{G}\left(\sigma_{G}\right) .
$$

Using the previous notations, the assertion that $\bar{P}(W)_{G}$ is a Poisson map can be restated as follows: for any given $G$-invariant functions $f_{i} \in C^{\infty}\left(W^{*}\right), i=1,2$, we have $\left\{f_{1 G}, f_{2 G}\right\}=\left\{f_{1}, f_{2}\right\}_{G}$. 
First, we will consider the case in which $f_{i} \in \mathrm{A}\left(W^{*}\right), i=1,2$. Then $f_{i}=$ $a_{i}+P\left(w_{i}\right)$, for some $a_{i} \in C^{\infty}(Q)$ and $w_{i} \in \Gamma(W), i=1,2$. The $G$-invariance of $f_{i}, i=1,2$, implies the $G$-invariance of $a_{i}$ and of $w_{i}$, that is, in particular, $w_{i} \in \Gamma^{G}(W), i=1,2$. Using this and the previous formulas we can show in a straightforward manner that $\left\{f_{1 G}, f_{2 G}\right\}=\left\{f_{1}, f_{2}\right\}_{G}$.

Second, we will consider the general case. Let $f_{i} \in C^{\infty}\left(W^{*}\right), i=1,2$, be $G$ invariant. Let $\sigma_{0} \in W^{*}$, say $\sigma_{0} \in W_{q_{0}}^{*}$. We can always find $\sigma \in \Gamma^{G}\left(W^{*}\right)$ such that $\sigma\left(q_{0}\right)=\sigma_{0}$. We can easily show that $f_{i}^{\sigma} \in C^{\infty}\left(W^{*}\right)$ is $G$-invariant, for $i=1,2$. Let $f_{i}^{\sigma}=a_{i}^{\sigma}+P\left(w_{i}^{\sigma}\right), i=1,2$. We can show easily, for $i=1,2$, that $\left(a_{i}^{\sigma}\right)_{G}=\left(a_{i G}\right)^{\sigma_{G}}$ and also that $\left(w_{i}^{\sigma}\right)_{G}=\left(w_{i G}\right)^{\sigma_{G}}$. Then we have

$$
\begin{aligned}
\left\{f_{1}, f_{2}\right\}\left(\sigma_{0}\right) & =\left\{f_{1}^{\sigma}, f_{2}^{\sigma}\right\}\left(\sigma_{0}\right)=\left\{f_{1}^{\sigma}, f_{2}^{\sigma}\right\}_{G}\left(\sigma_{0 G}\right)=\left\{\left(f_{1}^{\sigma}\right)_{G},\left(f_{2}^{\sigma}\right)_{G}\right\}\left(\sigma_{0 G}\right) \\
& =\left\{\left(f_{1 G}\right)^{\sigma_{G}},\left(f_{2 G}\right)^{\sigma_{G}}\right\}\left(\sigma_{0 G}\right)=\left\{f_{1 G}, f_{2 G}\right\}\left(\sigma_{0 G}\right) .
\end{aligned}
$$

We have proved the following

Theorem 8.2.1. $\bar{P}(W)_{G}: W^{*} \rightarrow W_{G}^{*}$ is a Poisson map.

This theorem establishes that Poisson reduction in the category $\mathfrak{L P}^{*}$, in particular cotangent bundle reduction, is dual to reduction in the category $\mathfrak{L P}$. The decomposition of the bracket as a sum of three brackets given in Montgomery, Marsden and Ratiu (1984) and Montgomery (1986) should be compared, via duality, with the decomposition of the reduced Lie bracket on sections of the reduced object of $\mathfrak{L P}$ given in Theorem 6.2.10. As we said before, a more detailed study of all this is being planned for future work.

\section{Acknowledgments.}

We thank Darryl Holm, Gerard Misiolek, Matt Perlmutter, Jürgen Scheurle and the referees for several helpful suggestions. 
8. The Category $\mathfrak{L P}^{*}$ and Poisson Geometry 


\section{Bibliography}

Abraham, R. and J. E. Marsden [1978], Foundations of Mechanics, Addison-Wesley, Second edition.

Abraham, R., J. E. Marsden and T. S. Ratiu [1988], Manifolds, Tensor Analysis and Applications, Applied Mathematical Sciences, 75, Springer-Verlag, New York, Second edition.

Arnold, V. I. [1989], Mathematical Methods of Classical Mechanics, Graduate Texts in Math., 60, Springer-Verlag, Second edition.

Bates, L. and E. Lerman [1997], Proper group actions and symplectic stratified spaces, Pacific J. Math., 181, 201-229.

Blaom, A.D. [2000], Reconstruction phases via Poisson reduction, Diff. Geom. and Appl., 12, 231-252.

Bloch, A. M.; Crouch, P. E. [1993] Nonholonomic and vakonomic control systems on Riemannian manifolds. Dynamics and control of mechanical systems, Fields Inst. Commun. 1, 25-52.

Bloch, A. M. and P. E. Crouch [1994], Reduction of Euler-Lagrange problems for constrained variational problems and relation with optimal control problems, in Proc. CDC, 33, 2584-2590.

Bloch, A. M. and P. Crouch [1995], Nonholonomic control systems on Riemannian manifolds., SIAM J. on Control, 33, 126-148. 
Bloch, A. M. and P. E. Crouch [1998], Newton's law and integrability of nonholonomic systems, SIAM J. Control Optim., 6, 2020-2039

Bloch, A. M. and P. E. Crouch [1999], Optimal control, optimization, and analytical mechanics, in Mathematical Control Theory, 268-321, J. Ballieul, Ed., Springer, New York.

Bloch, A. M., P. S. Krishnaprasad, J. E. Marsden and G. Sánchez De Alvarez [1992], Stabilization of rigid body dynamics by internal and external torques, Automatica, 28, 745-756.

Bloch, A. M., P. S. Krishnaprasad, J. E. Marsden and R. Murray [1996], Nonholonomic mechanical systems with symmetry, Arch. Rational Mech. Anal., 136, $21-99$.

Bloch, A. M., P. S. Krishnaprasad, J. E. Marsden and T. S. Ratiu [1996], The Euler-Poincaré equations and double bracket dissipation, Comm. Math. Phys., $\mathbf{1 7 5}, 1-42$.

Bloch, A. M., N. Leonard and J. E. Marsden, Controlled Lagrangians and the Stabilization of Mechanical Systems I: The First Matching Theorem, IEEE Trans. Automat. Control (to appear).

Bobenko, A. I. and Y. B. Suris [1999a], Discrete time Lagrangian mechanics on Lie groups, with an application to the Lagrange top, Commun. Math. Phys., 204, $147-188$.

Bobenko, A. I. and Y. B. Suris [1999b], Discrete Lagrangian reduction, discrete Euler-Poincaré equations, and semidirect products, Lett. Math. Phys., 49, 7993.

Bourbaki, N. [1983], Variétés differentielles et analytiqes. Fascicule de résultats, Diffusion C.C.L.S., Paris.

Bretherton, F. P. [1970], A note on Hamilton's principle for perfect fluids, J. Fluid Mech., 44, 19-31.

Cannas Da Silva, A. and A. Weinstein [1999], Geometric Models for Noncommutative Alebras, Amer. Math. Soc., Berkeley Mathematics Lecture Notes, 10.

Castrillón López, M., Ratiu, T. S. and Shkoller, S. [2000], Reduction in principal fiber bundles: Covariant Euler-Poincaré equations, Proc. Amer. Math. Soc., 128, 2155-2164.

Cendra, H., D. D. Holm, M. J. W. Hoyle and J. E. Marsden [1998], The MaxwellVlasov equations in Euler-Poincaré form, J. Math. Phys., 39, 3138-3157. 
Cendra, H., D. D. Holm, J. E. Marsden and T. S. Ratiu [1998], Lagrangian Reduction, the Euler-Poincaré Equations and Semidirect Products, Amer. Math. Soc. Transl., 186, 1-25.

Cendra, H., A. Ibort and J. E. Marsden [1987], Variational principal fiber bundles: a geometric theory of Clebsch potentials and Lin constraints, J. Geom. Phys., 4, 183-206.

Cendra, H. and J. E. Marsden [1987], Lin constraints, Clebsch potentials and variational principles, Physica D, 27, 63-89.

Cendra, H., J. E. Marsden and T. S. Ratiu [2000], Geometric Mechanics, Lagrangian Reduction and Nonholonomic Systems, in Mathematics Unlimited, SpringerVerlag, New York.

Courant, T. [1990], Dirac manifolds, Trans. Amer. Math. Soc., 319, 631-661.

De Azcárraga, J.E. and J.M. Izquierdo [1995] Lie Groups, Lie Algebras, Cohomology and Some Applications in Physics. Cambridge University Press.

Gamboa Saraví, R. E. and J. E. Solomin [2003], On the global version of EulerLagrange equations, J. Phys. A 36, 7301-7305.

Ge, Z. and J. E. Marsden [1988], Lie-Poisson integrators and Lie-Poisson HamiltonJacobi theory, Phys. Lett. A, 133, 134-139.

Guillemin, V. and S. Sternberg [1980], The moment map and collective motion, Ann. of Phys., 1278, 220-253.

Guillemin, V. and S. Sternberg [1984], Symplectic Techniques in Physics, Cambridge University Press.

Hamel, G. [1904], Die Lagrange-Eulerschen Gleichungen der Mechanik, Z. für Mathematik u. Physik, 50, 1-57.

Holm, D. D. [2000], Euler-Poincaré Dynamics of Ideal Micropolar Complex Fluids, (preprint).

Holm, D. D., J. E. Marsden and T. S. Ratiu [1986], The Hamiltonian structure of continuum mechanics in material, spatial and convective representations, in Séminaire de Mathématiques supérieurs, 100, 11-122, Les Presses de L'Univ. de Montréal.

Holm, D. D., J. E. Marsden and T. S. Ratiu [1998a], The Euler-Poincaré equations and semidirect products with applications to continuum theories, Adv. in Math., $137,1-8$. 
Holm, D. D., J. E. Marsden and T. S. Ratiu [1998b], Euler-Poincaré models of ideal fluids with nonlinear dispersion, Phys. Rev. Lett., 349, 4173-4177.

Jalnapurkar, S. M., M. Leok, J. E. Marsden and M. West [2000], Discrete Routh reduction; (preprint).

Jalnapurkar, S. M. and J. E. Marsden [1999], Stabilization of Relative Equilibria II, Regul. Chaotic Dyn., 3, 161-179.

Jalnapurkar, S. M. and J. E. Marsden [2000], Reduction of Hamilton's Variational Principle, Dynam. Stability Systems, 15, 287-318.

Kane, C, J. E. Marsden, M. Ortiz and M. West [2000], Variational Integrators and the Newmark Algorithm for Conservative and Dissipative Mechanical Systems, Int. J. Num. Math. Eng., 49, 1295-1325.

Kobayashi, S. and K. Nomizu [1963], Foundations of Differential Geometry, Wiley.

Kolár, I., P.W. Michor, and J. Slovák [1993] Natural Operators in Differential Geometry. Springer-Verlag.

Koon, W. S. and J. E. Marsden [1997a], Optimal control for holonomic and nonholonomic mechanical systems with symmetry and Lagrangian reduction, SIAM J. Control and Optim., 35, 901-929.

Koon, W. S. and J. E. Marsden [1997b], The geometric structure of nonholonomic mechanics, Proc. CDC, 36, 4856-4862.

Koon, W. S. and J. E. Marsden [1997c], The Hamiltonian and Lagrangian approaches to the dynamics of nonholonomic systems, Rep. Math. Phys., 40, 21-62.

Koon, W. S. and J. E. Marsden [1998], The Poisson reduction of nonholonomic mechanical systems, Reports on Math. Phys., 42, 101-134.

Lagrange, J. L. [1788], Mécanique Analytique, Chez la Veuve Desaint.

Leonard, N. E. and J. E. Marsden [1997], Stability and drift of underwater vehicle dynamics: mechanical systems with rigid motion symmetry, Physica D, 105, $130-162$.

Lewis, A. D. [1996] The geometry of the Gibbs-Appell equations and Gauss' principle of least constraint. Rep. Math. Phys. 38, 11-28.

Lewis, A. D. [2000] Towards $F=m a$ in a general setting for Lagrangian mechanics (To appear in Annales Henri Poincaré). 
Libermann, P. and C. M. Marle [1987], Symplectic Geometry and Analytical Mechanics, Kluwer Academic Publishers.

Mackenzie, K. [1987] Lie Groupoids and Lie Algebroids in Differntial Geometry. London Math. Soc. Lect. Note Series 124, Cambrigde Univ. Press.

Marsden, J. E. [1992], Lectures on Mechanics, London Math. Soc. Lecture Note Ser., 174, Cambridge University Press.

Marsden, J., G. Misiolek, M. Perlmutter and T. Ratiu [1998], Symplectic reduction for semidirect products and central extensions, Diff. Geom. and its Appl., 9, $173-212$.

Marsden, J. E., G. Misiolek, M. Perlmutter and T. S. Ratiu [2000], Reduction by stages and group extensions, Preprint.

Marsden, J. E., R. Montgomery, P. J. Morrison and W. B. Thompson [1986], Covariant Poisson brackets for classical fields, Annals of Physics, 169, 29-48.

Marsden, J. E., P. J. Morrison and A. Weinstein, The Hamiltonian structure of the BBGKY hierarchy equations, Contemp. Math., AMS, 28, 115-124.

Marsden, J. E., G. W. Patrick and S. Shkoller [1998], Mulltisymplectic Geometry, Variational Integrators and Nonlinear PDEs, Comm. Math. Phys., 199, 351-395.

Marsden, J. E., S. Pekarsky and S. Shkoller [1999], Discrete Euler-Poincaré and Lie-Poisson equations, Nonlinearity, 12, 1647-1662.

Marsden, J. E., S. Pekarsky and S. Shkoller [2000], Symmetry reduction of discrete Lagrangian mechanics on Lie groups, J. Geom. and Phys., 36, 140-151.

Marsden, J. E. and M. Perlmutter [2000], The Orbit Bundle Picture of Cotangent Bundle Reduction, C. R. Math. Rep. Acad. Sci. Canada, 22, 33-54.

Marsden, J. E. and T. Ratiu [1986], Reduction of Poisson Manifolds, Lett. in Math. Phys., 11, 161-170.

Marsden, J. E. and T. S. Ratiu [1999], Introduction to Mechanics and Symmetry, Texts in Applied Mathematics, 17; Second Edition, 1999.

Marsden, J. E., T. S. Ratiu and J. Scheurle [2000], Reduction theory and the Lagrange-Routh equations, J. Math. Phys., 41, 3379-3429.

Marsden, J. E., T. Ratiu and S. Shkoller [2000], The geometry and analysis of the averaged Euler equations and a new diffeomorphism group, Geom. Funct. Anal., 10, 582-599. 
Marsden, J. E., T. S. Ratiu and A. Weinstein [1984], Semi-direct products and reduction in mechanics, Trans. Amer. Math. Soc., 281, 147-177.

Marsden, J. E., T. S. Ratiu and A. Weinstein [1984], Reduction and Hamiltonian structures on duals of semidirect product Lie Algebras, Amer. Math. Soc., Providence, RI, Contemp. Math., 28, 55-100.

Marsden, J. E. and J. Scheurle [1993], Lagrangian reduction and the double spherical pendulum, ZAMP, 44, 17-43.

Marsden, J. E. and J. Scheurle [1993], The reduced Euler-Lagrange equations, Fields Inst. Commun., 1, 139-164.

Marsden, J. E. and S. Shkoller [1999], Multisymplectic geometry, covariant Hamiltonians and water waves, Math. Proc. Camb. Phil. Soc., 125, 553-575.

Marsden, J. E. and A. Weinstein [1974], Reduction of symplectic manifolds with symmetry, Rep. Math. Phys., 5, 121-130.

Meyer, K. R. [1973], Symmetries and integrals in mechanics, in Dynamical Systems, M. Peixoto, ed., 259-273, Academic Press.

Marsden, J. E., R. Montgomery and T. S. Ratiu [1990], Reduction, symmetry and phases in mechanics, Amer. Math. Soc., Providence, RI Memoirs, 436.

Montgomery, R. [1984], Canonical formulations of a particle in a Yang-Mills field, Lett. Math. Phys., 8, 59-67.

Montgomery, R. [1986], The Bundle Picture in Mechanics, Ph.D. Thesis, University of California Berkeley.

Montgomery, R. [1990], Isoholonomic problems and some applications, Comm. Math Phys., 128, 565-592.

Montgomery, R. [1993], Gauge theory of the falling cat, Fields Inst. Commun., 1, 193-218.

Montgomery, R., J. E. Marsden and T. S. Ratiu [1984], Gauged Lie-Poisson structures, Contemp. Math., 28, 101-114, Amer. Math. Soc., Providence, RI.

Moser, J. and A. P. Veselov [1991], Discrete versions of some classical integrable systems and factorization of matrix polynomials, Comm. Math. Phys., 139, 217243.

Ortega, J.-P. [1998], Symmetry, Reduction and Stability in Hamiltonian Systems, Ph.D. Thesis, University of California Santa Cruz. 
Ortega, J.-P. and T. S. Ratiu [1997], Persistence and smoothness of critical relative elements in Hamiltonian systems with symmetry, C. R. Acad. Sci. Paris Sér. I Math., 325, 1107-1111.

Ortega, J.-p. and T. S. Ratiu [2001], Hamiltonian Singular Reduction, Birkhäuser, Progress in Math.; (to appear).

Palais, R. S. [1961a], Equivalence of nearby differentiable actions of a compact group, Bull. Amer. Math. Soc., 67, 362-364.

Palais, R. S. [1961b], On the existence of slices for actions of non-compact Lie groups, Ann. of Math., 73, 295-323.

Poincaré, H. [1901a], Sur une forme nouvelle des équations de la méchanique, $C$. R. Acad. Sci., 132, 369-371.

Poincaré, H. [1901b], Sur la stabilité de l'équilibre des figures piriformes affectées par une masse fluide en rotation, Philosophical Transactions A, 198, 333-373.

Ratiu, T. S. [1980a], The Euler-Poisson Equations and Integrability, Ph.D. Thesis, University of California at Berkeley.

Ratiu, T. S. [1980b], The motion of the free $n$-dimensional rigid body, Indiana Univ. Math. Journ., 29, 609-629.

Ratiu, T. S. [1980c], Involution theorems, in Geometric Methods in Mathematical Physics, G. Kaiser and J. Marsden, eds., Springer Lecture Notes, 775, 219-257.

Ratiu, T. S. [1981], Euler-Poisson equations on Lie algebras and the $N$-dimensional heavy rigid body, Proc. Natl. Acad. Sci., USA, 78, 1327-1328.

Ratiu, T. S. [1982a], Euler-Poisson equations on Lie algebras and the $N$ dimensional heavy rigid body, Amer. J. Math., 104, 409-448, 1337.

Ratiu, T. S. [1982b], The Lie algebraic interpretation of the complete integrability of the Rosochatius system, in Mathematical Methods in Hydrodynamics and Integrability in Dynamical Systems (La Jolla Institute, 1981), AIP Conference Proceedings, 88, 109-116.

Routh, E. J. [1877], Stability of a given state of motion, Halsted Press, New York; Reprinted in Stability of Motion (1975), A. T. Fuller ed.

Sjamaar, R. and E. Lerman [1991], Stratified symplectic spaces and reduction, Ann. of Math., 134, 375-422. 
Sternberg, S. [1977], Minimal coupling and the symplectic mechanics of a classical particle in the presence of a Yang-Mills field, Proc. Nat. Acad. Sci., 74, 52535254 .

Tulczyjew, W. M. [1977], The Legendre transformation, Ann. Inst. Poincaré, 27, $101-114$.

Vershik, A. M. and V. Gershkovich [1988], Ya. Nonholonomic problems and the theory of distributions. Acta Appl. Math. 12, 181-209.

Vershik, A. M. and V. Ya Gershkovich [1994], Non-holonomic Riemannian manifolds, in Dynamical Systems 7, Springer, Encyclopaedia of Mathematics, 16.

Veselov, A. P. [1988], Integrable discrete-time systems and difference operators, Funct. An. and Appl., 22, 83-94.

Veselov, A. P. [1991], Integrable Lagrangian correspondences and the factorization of matrix polynomials, Funct. An. and Appl., 25, 112-123.

Weinstein, A. [1978], A universal phase space for particles in Yang-Mills fields, Lett. Math. Phys., 2, 417-420.

Weinstein, A. [1978], Bifurcations and Hamilton's principle, Math. Zeit., 159, 235248.

Weinstein, A. [1983a], The local structure of Poisson manifolds, J. of Diff. Geom., 18, 523-557.

Weinstein, A. [1983b], Sophus Lie and symplectic geometry, Expo. Math., 1, 95-96.

Weinstein, A. [1996], Lagrangian Mechanics and Groupoids, Fields Inst. Commun., 7, 207-231.

Weinstein, A. [1998] Poisson geometry, Diff. Geom. Appl. 9, 213-238.

Wendlandt, J. M. and J. E. Marsden [1997], Mechanical integrators derived from a discrete variational principle, Physica D, 106, 223-246.

Wong, S. K. [1970], Field and particle equations for the classical Yang-Mills field and particles with isotopic spin, Il Nuovo Cimento, LXV, 689-694. 